\title{
Understanding and Designing Attention for Dual-Screen Media
}

\author{
Neate, Timothy
}

How to cite:

Neate, Timothy (2017) Understanding and Designing Attention for Dual-Screen Media. Doctoral thesis, Swansea University.

http://cronfa.swan.ac.uk/Record/cronfa39010

Use policy:

This item is brought to you by Swansea University. Any person downloading material is agreeing to abide by the terms of the repository licence: copies of full text items may be used or reproduced in any format or medium, without prior permission for personal research or study, educational or non-commercial purposes only. The copyright for any work remains with the original author unless otherwise specified. The full-text must not be sold in any format or medium without the formal permission of the copyright holder. Permission for multiple reproductions should be obtained from the original author.

Authors are personally responsible for adhering to copyright and publisher restrictions when uploading content to the repository.

Please link to the metadata record in the Swansea University repository, Cronfa (link given in the citation reference above.)

http://www.swansea.ac.uk/library/researchsupport/ris-support/ 


\section{Understanding and Designing Attention for Dual-Screen Media}

TIMOTHY NEATE

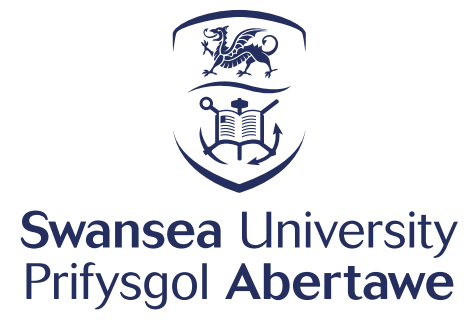

Submitted in fulfilment of the requirements for the Degree of Doctor of Philosophy

Future Interaction Technology Laboratory

Department of Computer Science

College of Science

Swansea University 



\section{Declaration}

This work has not been previously accepted in substance for any degree and is not being concurrently submitted in candidature for any degree. The work in this thesis has, however, undergone peer review and has been presented at major international venues. The publications noted below were prepared for submission under the guidance of Professor Matt Jones and Dr. Michael Evans.

1. Timothy Neate, Matt Jones, Michael Evans. Future Media: The Role of HCI in Broadcast. In Proceedings of York Doctoral Symposium on Computer Science and Electronics (YDS), York, United Kingdom, 2014.

2. Timothy Neate, Matt Jones, Michael Evans. Mediating Attention for Second Screen Companion Content. In Proceedings of the 33rd Annual ACM Conference on Human Factors in Computing Systems (CHI), Seoul, South Korea, 2015.

3. Timothy Neate, Matt Jones, Michael Evans. Designing Attention for Multiscreen TV Experiences. In Proceedings of the 2015 British HCI Conference (British HCI), Lincoln, United Kingdom, 2015.

4. Timothy Neate, Michael Evans, Matt Jones. Designing Visual Complexity for Dual-screen Media. In Proceedings of the 34th Annual ACM Conference on Human Factors in Computing Systems (CHI), San Jose, USA, 2016.

5. Timothy Neate, Matt Jones, Michael Evans. Towards Cross-device Harmony. In Cross-Surface '16 workshop, in conjunction with ACM CHI, San Jose, USA, 2016. 
6. Timothy Neate, Matt Jones, Michael Evans. Interdevice Media: Choreographing Content to Maximize Viewer Engagement. In IEEE Computer (49)12:3239, 2016. Also available as BBC White Paper 327.

7. Timothy Neate, Matt Jones, Michael Evans. Cross-Device Media: A Review of Second Screening and Multi-Device Television. Personal and Ubiquitous Computing, 2017.

8. Timothy Neate, Michael Evans, Matt Jones. Enhancing Interaction with Dual-Screen Television Through Display Commonalities. 5th Annual ACM International Conference on Interactive Experiences for Television and Online Video (TVX) Hilversum, The Netherlands, 2017.

Statement 1 - This thesis is the result of my own investigations, except where otherwise stated. Other sources are acknowledged by references in the text which correspond to the appended bibliography.

Statement 2 - I hereby give consent for my thesis, if accepted, to be available for photocopying and for inter-library loans after expiry of a bar on access approved by Swansea University. 


\section{Abstract}

Recently there has been a transformative shift towards engaging with mobile devices while watching television. Content creators, therefore, wish to create applications to support these behaviours to provide more engaging multi-device TV. Currently, their designs do not reflect the subtle variations in viewer attention, our physiological capabilities, or the additional mental effort such scenarios imply. We investigate this in two primary ways: by further understanding the current issues faced by users when dual-screening, and by designing a series of technological interventions for managing cross-device attention. First, we conduct two studies to better understand the user experience of second screening. Through a large-scale online questionnaire and a series of interviews we document the problems faced by users when second screening and how they compensate and mitigate for missing content when engaging with mobile devices. We then conduct an investigation to explore the effect of dual-screen visual complexity in terms of objective and subjective experience of participants when exposed to content of varying complexity across two screens.

For our technological interventions, we first investigate how visual complexity on a mobile device may be varied to account for the perceived complexity of TV material by loading textual material at varying levels of complexity. We explore the tradeoff of user autonomy and content creator control by contrasting the effects of users adjusting the complexity themselves, and automatic adjustment around heuristics. Then, we consider how different audio-visual stimuli may be used to direct a user's attention between screens at key moments. Finally, we explore how we can support users to reduce the switching costs and cognitive effort associated with engaging with cross-device media mirroring unattended visual information in the experience on an attended screen. Throughout the thesis we show that many of our interventions are a beneficial state of the art and form a series of guidelines for each. The thesis concludes by offering an outline of our contributions and a framework for others to extend our work. 


\section{Acknowledgements}

First and foremost, I would like to thank my two primary supervisors Matt Jones and Michael Evans. Matt, your guidance and support throughout my PhD made it a thoroughly enjoyable experience and shaped me as a researcher. Mike, your feedback, optimism, and unique 'BBC' perspective was invaluable and completely opened up the research I conducted. My thesis advisor, Rita Borgo, should also be noted as a person who gave invaluable advice throughout the progression milestones of my $\mathrm{PhD}$ studies. Her replacement, Simon Robinson, also gave me very useful feedback in the latter stages of my write-up. I would like to express gratitude to my external examiners, Yvonne Rogers and Roderick Murray-Smith, for an enjoyable viva and for their feedback on my thesis.

I thank my fellow researchers who became good friends during my $\mathrm{PhD}$ and helped make it an enjoyable experience, especially Cameron Steer, Liam Betsworth, Deepak Sahoo, Jen Pearson, Jean Razafindrakoto, Dean Thomas, Emma James, Tom Owen, Patrick Oladimeji and Karen Li. Outside of Swansea, I also thank my childhood friends Mathew Andrews, Lee Hind, Ethan Flynn-Harding and Kristie Sylvester for their camaraderie.

In terms of financial support, my $\mathrm{PhD}$ work was fully funded by the College of Science at Swansea, through an EPSRC DTA and BBC R\&D, who provided additional funds, all of which I am very thankful for. I thank BBC R\&D further for hosting me to run user studies a number of times during my PhD. In particular, Elizabeth Samson for her technical assistance when running user studies.

At the core of this research was my participants. I appreciate their time greatly and thank them for their insight throughout this my $\mathrm{PhD}$ work. Finally, I would like to thank those dearest to me - my parents, Laurance and Lindsey Neate, for being open-minded and very supportive of everything I do, and my partner, Alena Denisova, for her constant support. 


\section{Contents}

1 INTRODUCTION

1.1 Second Screening and Companion Content . . . . . . . . . . 3

1.2 The Challenges of Cross-Device Experiences . . . . . . . . . 5

1.3 Research Context and Author Contribution . . . . . . . . . . 7

1.4 Structure and Thesis Contributions . . . . . . . . . . . . 8

2 BACKGROUND 13

2.1 Multi-Display Contexts . . . . . . . . . . . . . . 13

2.1.1 Peripheral and Ambient Displays . . . . . . . . . . . 16

2.1.2 Multi-Device Computing . . . . . . . . . . . 17

2.1 .3 Origins of Multi-Screen TV . . . . . . . . . . . 18

2.1 .4 Second Screening . . . . . . . . . . . . . . 20

2.2 The Infrastructure of the Multi-Screen Home . . . . . . . . . . . . . 22

2.2.1 Object-Based Broadcasting . . . . . . . . . . . . . 24

2.2.2 The Increasing Ambiguity of the Second Screen . . . . . . . 26

2.3 From Second Screening to Dual-Screen Experiences . . . . . . . . . 27

2.3.1 Publicly Available Applications . . . . . . . . . . . . 27

2.3.2 Commercial and Prototype Companions: A Categorisation . 30

2.3.3 Extending the Remote Control . . . . . . . . . . . . . . . 34

2.3.4 Authorship, Sustainability and Artistic License . . . . . . . . 35

2.4 Future Considerations . . . . . . . . . . . . . . 37

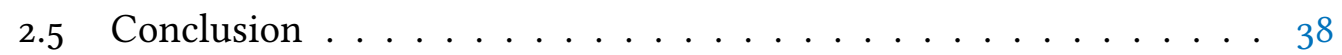

3 METHODOLOGY 39

$3.1 \quad$ Research Objective and Questions . . . . . . . . . . . . . . 39

3.2 Research Overview . . . . . . . . . . . . . . 40

3.2.1 Research Methods . . . . . . . . . . . . . . 41 
3.2.2 Research Environment . . . . . . . . . . . . 41

3.2.3 Research Scope . . . . . . . . . . . . . . . 43

3.3 Conclusions . . . . . . . . . . . . . . . 43

4 BOTTLENECKS OF CROSS-DEVICE SCENARIOS 45

4.1 Attention Bottlenecks in the Literature . . . . . . . . . . 45

4.1.1 Studies of Cross-Device Attention . . . . . . . . . . 51

4.1 .2 Display Discontinuities . . . . . . . . . . . 54

4.1 .3 Information Overload . . . . . . . . . . . 55

4.2 The Viewers' Perspective: An Online Survey . . . . . . . . . . . 56

4.2.1 Experiment Description and Participants . . . . . . . . 56

4.2.2 Quantitative Results . . . . . . . . . . . . . 61

4.2 .3 Qualitative Results . . . . . . . . . . . . . . 62

4.3 Discussion ......................... 64

4.4 Dual-screen Usage Interview . . . . . . . . . . . . . . 66

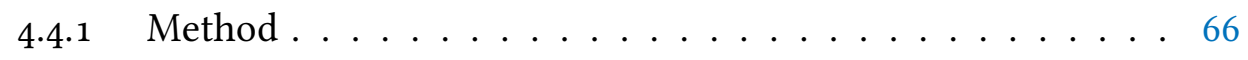

$4.4 .2 \quad$ Results . . . . . . . . . . . . . . 68

4.4 .3 Discussion ...................... 71

4.5 Implications and Design Recommendations . . . . . . . . . 71

4.6 Conclusion . . . . . . . . . . . . . . . . . 74

5 QUANTIFYING CROSS-DEVICE COMPLEXITY 77

5.1 Quantifying Visual Complexity . . . . . . . . . . . . 78

5.1.1 Visual Attention for Dual-Screen TV . . . . . . . . . 79

5.2 Study of Varying Cross-Device Visual Complexity . . . . . . . . . . 81

5.2.1 Participants ................... 81

5.2.2 Study Environment . . . . . . . . . . . . . 82

$5.2 .3 \quad$ Study Procedure . . . . . . . . . . . . . 82 
5.2.4 Stimuli and Tablet Content Design . . . . . . . . . . 83

5.2.5 Measures and Validation Techniques . . . . . . . . 85

5.3 Results ......................... 88

$5.3 .1 \quad$ Visual Data . . . . . . . . . . . . . . 88

5.3.2 Experimental Questionnaires ............. 91

5.3 .3 Post-study Interview . . . . . . . . . . . . . . 92

5.4 Discussion . . . . . . . . . . . . . . . . 94

5.5 Design Implications and Conclusion . . . . . . . . . . . . . . 97

6 COMPENSATING FOR CROSS-DEVICE COMPLEXITY 99

6.1 Adaptive and Adaptable UI . . . . . . . . . . . . . . . . . . 100

6.2 Adaptive and Adaptable UI: TV for HCI Approaches . . . . . . . . . 101

6.2.1 Recommender Systems for TV . . . . . . . . . . . . . 101

6.2.2 Perceptive Media ................. 102

6.3 Complexity Curation and Adjustment . . . . . . . . . . . . 103

6.3.1 Device-Driven Complexity Curation . . . . . . . . . 104

6.3.2 Complexity Adaption by Users . . . . . . . . . . . . . 105

6.4 Empirical Study of Complexity Compensation _ . . . . . . . . 106

6.4.1 Procedure ......................... 107

6.4.2 Stimuli and Tablet Content Design . . . . . . . . . . 107

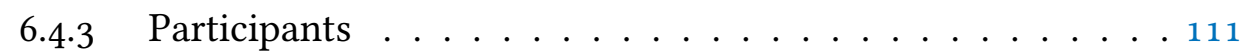

6.4.4 Measures and Validation Techniques . . . . . . . . . . 111

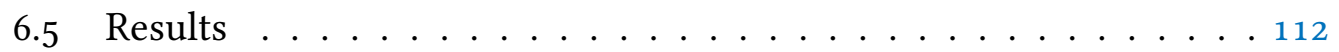

6.5.1 Questionnaire Data .................. 112

6.5 .2 Interaction Data . . . . . . . . . . . . . . . 114

6.5 .3 Post-study Interview . . . . . . . . . . . . . . 115

6.6 Discussion .......................... 117 
6.7 Design Considerations and Further Work . . . . . . . . . . . . 118

6.8 Conclusions . . . . . . . . . . . . . . . . . . 120

7 MEDIATING ATTENTION 123

7.1 Attention Management with Additional Stimuli . . . . . . . . . 124

7.1.1 Visual Notifications . . . . . . . . . . . . . . 125

7.1.2 Auditory Notifications . . . . . . . . . . . . 126

7.2 Attention Mediation Methods . . . . . . . . . . . . . 127

7.2.1 Design of Stimuli . . . . . . . . . . . . . . 128

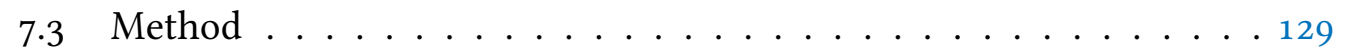

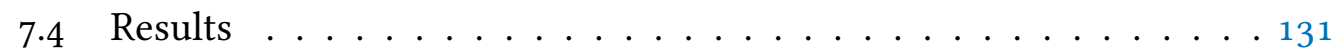

7.4.1 Objective Results . . . . . . . . . . . . . . 131

$7.4 .2 \quad$ Subjective Results . . . . . . . . . . . . . . 133

7.5 Discussion . . . . . . . . . . . . . . . 135

7.6 Design Implications and Conclusion . . . . . . . . . . . . 136

8 DisPlay COMMONALITIES 139

8.1 Screen Mirroring in the Connected Home . . . . . . . . . . . . 140

8.2 Display Commonalities Design Space . . . . . . . . . . . . . 142

8.3 Focus Group with Practitioners . . . . . . . . . . . . . . . . . 146

$8.3 .1 \quad$ Participants ........................ 146

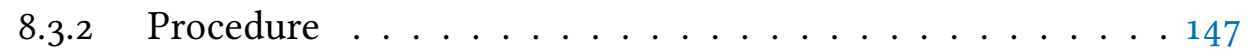

$8.3 .3 \quad$ Results . . . . . . . . . . . . . . . . 148

8.3.4 Reflections on Focus Group . . . . . . . . . . . . 150

8.4 Empirical Investigation of Commonalities . . . . . . . . . . . 150

8.4.1 Participants and Study Environment . . . . . . . . . 151

8.4 .2 Study Procedure . . . . . . . . . . . . . . 153

8.4.3 Measures and Motivations . . . . . . . . . . . 156 
8.5 Results . . . . . . . . . . . . . . . 157

8.5 .1 Post-Clip Questionnaires . . . . . . . . . . . . 158

8.5.2 Post-Study Questionnaires . . . . . . . . . . . . 161

8.5 .3 Device Log Data . . . . . . . . . . . . . . . . . 162

8.5 .4 Post-Study Interviews . . . . . . . . . . . . . . 164

8.5 .5 Discussion ..................... 167

8.6 Implications, Feasibility and the Future . . . . . . . . . . . . 169

8.7 Conclusion . . . . . . . . . . . . . . . 172

9 CONCLUSIONS AND FUTURE WORK 175

9.1 Contributions and Reflection on Research Questions . . . . . . . . 176

9.2 A Generic Framework for Designing Interventions . . . . . . . . . 182

9.2.1 Autonomy of Intervention . . . . . . . . . . . . . . 184

9.3 Limitations of This Work . . . . . . . . . . . . . . 186

9.4 Future Work . . . . . . . . . . . . . . . . 187

9.5 Concluding Remarks _. . . . . . . . . . . . . . . 190

APPENDIX A: ONLINE QUESTIONNAIRE 192

APPENDIX B: VISUAL COMPLEXITY QUESTIONNAIRE 199

APPENDIX B: ONLINE QUESTIONNAIRE 207

APPENDix D: MEDiATING ATTENTION QUESTIONNAIRE 214

APPENDIX E: DiSPLAY COMMONALITIES QUESTIONNAIRE 223

APPENDIX F: RESEARCH CONSENT FORMS 232

$\begin{array}{ll}\text { BIBLIOGRAPHY } & 245\end{array}$ 


\section{List of Figures}

Figure 1

Figure 2

Figure 3

Figure 4

Figure 5

Figure 6

Figure 7

Figure 8

Figure 9

Figure 10

Figure 11

Figure 12

Figure 13

Figure 14

Figure 15

Figure 16

Figure 17

Figure 18

Figure 19

Figure 20

Figure 21

Figure 22
The many forms of second screening $\ldots \ldots \ldots . . \ldots 2$ Workstation monitor . . . . . . . . . . . . . 14

Object-based broadcasting paradigm . . . . . . . . . 25

Divided attention in cross-device scenario . . . . . . . 47

Field of view . . . . . . . . . . . . . . . . 49

Map of interactivity and concentration in the second-screen use case . . . . . . . . . . . . . . . . 51

Wordcloud of frequently noted phrases . . . . . . . 63

Video observation from three angles . . . . . . . . . . 82

The graphical second screen complexity levels used in the experiment . . . . . . . . . . . . 84

Visual Complexity: glances per condition . . . . . . . . 89

Time participants engaged with content . . . . . . . 90

Complexity curation process . . . . . . . . . . . 105

Adapting the complexity manually . . . . . . . . . . 106

Screens from a navigable application $\ldots \ldots \ldots . . \ldots 108$

Frequency of all adjustments . . . . . . . . . . . . 114

Frequency of first choice made by participants . . . . . . 115

Example of auditory icons and earcons . . . . . . . . . 127

Example content used mediating attention study . . . . . . 129

Participant perspectives of mediating attention study . . . 131

Participants' reaction times in ascending order . . . . . . . 132

Histogram of response times . . . . . . . . . . . 133

Examples of mirroring . . . . . . . . . . . . . 141 
Figure 23 Initial design space discussed with practitioners . . . . 145

Figure 24 Conditions represented by 'Wild China' application . . . . 152

Figure $25 \quad$ A user utilising display commonalities . . . . . . . . 153

Figure $26 \quad$ Examples of a companion application . . . . . . . . 155

Figure $27 \quad$ Mean interactions per condition . . . . . . . . . 163

Figure $28 \quad$ Mean number of sites visited per session . . . . . . . . 164

Figure 29 Demonstrator application for British Science Festival . . . 171

Figure $30 \quad$ Bottom-up hierarchy of designing attention management techniques . . . . . . . . . . . . 183

Figure $31 \quad$ Map of control in interventions . . . . . . . . . . 185 


\section{List of Tables}

Table 1

Table 2

Table 3

Table 4

Table 5

Table 6

Table 7

Table 8

Table 9

Table 10

Table 11

Table 12

Table 13

Table 14
Data from the online questionnaire $\ldots . . . .660$

Table of clips used in the visual complexity experiment . . 83

Example of the data generated from the video analysis . . 86

Perceived effects of varying complexity . . . . . . . 91

Clips used in complexity adjustment experiment . . . . . . 109

Participant agreement for level of detail that allowed them

to take in information . . . . . . . . . . . . 113

Frequency of first interaction when presented with a new

view . . . . . . . . . . . . . . . 116

Attention mediation methods . . . . . . . . . . . . 128

Participants reported attention gained for methods . . . . . 134

Participants reported preference for attention mediation . 135

Designer participant demographics . . . . . . . . 147

Table of clips used in to evaluate display commonalities . . 154

Commonality methods - participants' subjective impressions . . . . . . . . . . . . . . . . 159

Post-study questionnaire for commonalities experiment . . 162 


\section{CHAPTER ONE}

\section{Introduction}

Emerging technologies can invoke feelings of joy, exuberance and uncertainty, altering many facets of our usage with existing devices as the adoption of new technologies becomes more prominent. Mobile devices with capacitive touch screens, such as smartphones and tablets have, in a few short years, transformed the way we interact with technology and our surroundings. They proliferate and disrupt almost every commonplace routine, serving as an anchor to our personal digital worlds, and a handheld portal by which we can seemingly accomplish any typical computing task, and more. Around our mobile devices sits a myriad of other technologies which users concurrently engage with. Many of these cross-device interactions are rich, and well supported; others are of limited success.

One highly prominent, and omnipresent example of mobile devices spreading into an existing technology context, is the way we watch television accompanied with mobile devices (see Figure 1). We regularly engage with second screens while watching programmes, so much so that it has become a part of the living room landscape, transforming the way we engage with our televisions.

The power of our handheld devices allows us to engage in televised debates through social media, search characters we see in programmes and keep in touch with our social networks as the events in the programme unfold. The majority of these interactions are borne from users engaging with mobile devices while watching TV, and are not designed by the content creators. In such cases, the devices work together 
in isolation from each other. Of late, however, many broadcasters and application developers wish to extend this scenario to make television content more engaging, by creating applications which run in tandem with programmes. Such cross-device experiences can take the form of time-synchronised tangential information [101], or even game-like 'play-along' aspects (for example, guessing the price of antiques in a programme about collectables [146]).

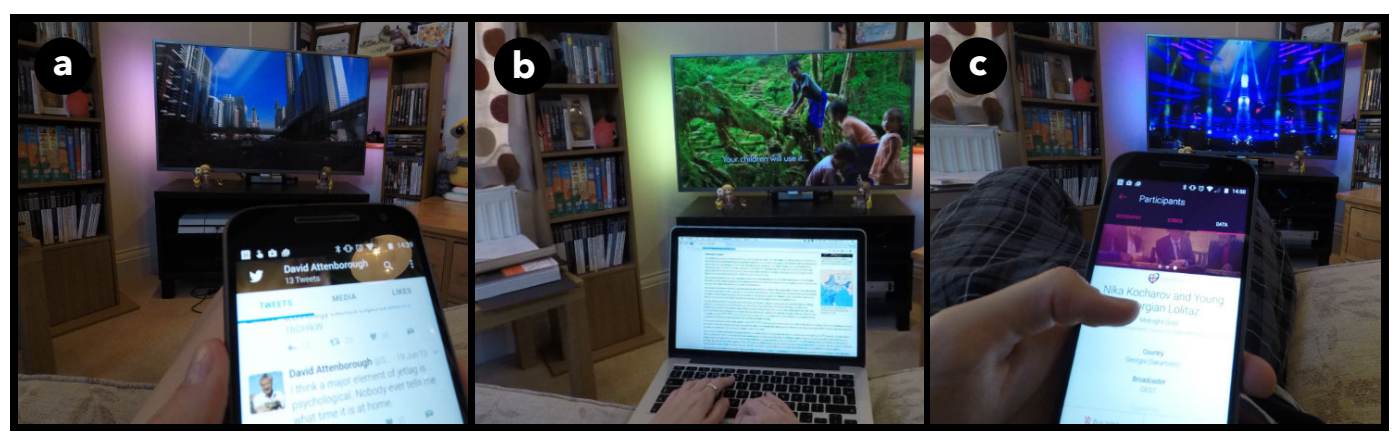

Figure 1: Second screening takes many forms: one may engage with social media while watching a programme on a mobile device, as in a); read more in-depth information on a laptop, like in b); or download a dedicated application to get programme-related information and vote on live events, as in c).

Such cross-device experiences, however, do not take into account the nuances of human attention, and the pronounced mental effort such scenarios require us to engage with. Previous work in the academic literature suggests that there are some clear shortfalls in how users' attention is managed in these cross-device media contexts. This is a highly pertinent area of investigation, not only because the second screen use case is so widespread, but also because this strong increase of people interacting with devices while watching TV positions it as a potential exemplar of multi-device interaction, and a context for pushing the frontiers of design for multi-screen and multi-device contexts. 
Attention for cross-device content, then, is currently undesigned - broadcasters and application developers do not have a good understanding of cross-device attention, nor do they have any tools or techniques to consider its design. Therefore, in this thesis, we aim to understand and design attention for multi-device media, towards fostering more harmonious experiences in which the content streams (TV or personal device) are complementary. Invoked by the shortcomings of the use cases outlined by the literature, we conduct empirical studies to understand the objective and subjective impact of the dual-screen use case; then, design interventions to allow designers to consider how they may compensate for such phenomena. We investigate interventions, which give users varying control over their experience empirically from a qualitative and quantitative view, to explore their costs and benefits, and outline design guidelines for cross-device media.

\subsection{SECOND SCREENING AND COMPANION CONTENT}

Since the middle of the 21 st century, the television has become a focal point of the modern home, often serving as a centre piece, and a shared focus for families to gather around to unwind and reflect on their day. Even now, in the smartest of homes, its fundamental component (a large shared display for video consumption) normally remains a centre-point of our living rooms, albeit in a more internetenabled way.

The large uptake of mobile devices in the living room has seen a large diversity of second screen interactions, which are mixed in terms of how related they are to the programme at hand. Rooksby et al. [122] and D'heer et al. [37], when studying how people use their mobile devices while watching television, found that their usage is highly complex and nuanced - sometimes totally unrelated to the programme; 
sometimes totally related; often a mixture of both. The mobile device is highly personal and requires highly focused attention - Holz et al. [67] when studying how users use their second devices while watching television found that the device often acts as the primary screen, and the users tend to focus on this, switching back to the television when it piques their attention.

Statistics show that a significant proportion of television viewing is accompanied by mobile device usage. In fact, this is quickly becoming one of the most ubiquitous multi-device use cases. A recent (2015) report suggests that $87 \%$ of US consumers engage with second screens while watching television [90]. This statistic appears to show growth since Google's 2012 consumer study [54], which found this figure to be $77 \%$,and that this was predominantly smartphones (49\%) and laptops/PCs $(34 \%)$.

Though in many cases the digital worlds are disparate and unrelated, and users engage with material totally unrelated to the programme, much content browsing is also related to the television. The previously mentioned Google report, for instance, suggests that $22 \%$ of simultaneous use is complementary - typically with a tablet (40\%) or a smartphone (38\%) [54]. Such interactions have their origins in the early days of television - we have always liked to get involved with the events on screen. Viewer-television interactions, such as phone-ins and letter, have now been pushed into the digital age. They manifest as reflections of their former selves through digital mediums such as social media, people tweeting along with live debates, for example. Now, in more recent times, this explosion of internet-equipped, interactive, powerful devices into our living rooms has borne new exiting ways for content creators to support these behaviours in real time. 
To extend such second screen behaviours, many popular programmes now have an associated second screen app, which is designed to run alongside the programme, allowing for real time feedback and interaction with a live programme. Viewer behaviours, such as interacting with social media while watching television are now increasingly supported by dedicated second screen application, designed either by the broadcaster or by an independent application developer. Such applications allow for the extension of their second screen engagement - for instance, the Britain's Got Talent Application allowed users to play-along with on-line events [112] - vote off acts, and join in polls - behaviours once enabled through social media. Essentially, such applications lower the cost of the user searching for specific online content by providing information or content when relevant - be it the previously mentioned viewer-programme interaction, or simply relevant trivia and programme-related materials (c.f. in the Breaking Bad Story Sync app [101]) to allow a user to better frame the programme.

When a user engages with a second screen while watching television - be it driven by a second screen application, or by individual query - there are a number of UX (user experience) bottlenecks. The ergonomics of the use case, for instance, generally suggest that when the user is engaging with their mobile device, they are typically gazing downwards into their laps - monitoring the audio feed of the television. In addition, the simultaneous perception of auditory and textual information is, due to the way we process information, highly problematic [123]. In second screen scenarios users tend to switch attention, as opposed to dividing between both screens. Typically, users rest the device on their lap, in their peripheral - 
creating a visual congruence between the foci. This means they must constantly check the mobile device for new content when focusing on the TV, and monitor the auditory stream of the TV for points that pique their interest.

Much research of late has outlined the heightened attention required to engage with the multiple attention streams - Basapur et al. [10] for instance, note when trailing a companion application in a deployment that such applications very much support 'active TV' - that they are quite cognitively involving and perhaps something that someone would not use to 'unwind'. Similarly, this notion of information overload was observed by Geerts et al. [53] who found that viewers were often overwhelmed with the stimuli, and had to return to it later when the pace of the main programme had slowed down (and, therefore, when the companion material had become less relevant).

The way the users' attention is managed is a key driver in much of the HCI research around this field of late - for example, recent studies by Holmes et al. [66] and Brown et al. [23] has looked to investigate how cross device attention is managed by a user in a given scenario. Much of this research, which we study in more detail in the background of this thesis (Chapter 2), suggests strong attention constraints to this scenario, which are not yet understood or considered. In order to make multiple displays truly complementary, we believe that it is essential to understand how to design for the likely patterns of attention distribution between the foci of the two (or more) screens. The focus of this thesis, then, is to better understand the attention constraints of inter-device media, using this information to enhance the design of cross-device attention through interventions and informed design of second screen content. 
This PhD research was supported by BBC R\&D User Experience Research Partnership (UXRP) and co-supervised by a senior researcher working at the department. As such, much of the work presented in this thesis is - though driven by literature and the HCI community - also informed by content designers and researchers at the BBC. A core research theme of the UXRP was to consider the "User Experience for the New Broadcasting System".

The work at the BBC had (prior to this PhD's commencement) mostly focused on understanding dual-screen attention for cross device experiences (c.f the work of Brown et al. [23]). Here, we saw a strong opportunity to extend this work, ultimately framed and facilitated in the BBC's broader context of object-based broadcasting (OBB) (see [6]) and multi-device interactive experiences. We were, then, given a highly broad remit to collaborate and given access to the rich perspectives of the BBC to drive forward academic research.

All work presented in this thesis was undertaken by the thesis author under the guidance of two primary supervisors: ideation, creation of software prototypes, study design, study running, data analysis and writing. Studies presented in this thesis were conducted within the ethics framework of Swansea University Computer Science Department (see Appendix F for consent forms and participants' Bill of Rights). All other noted work is properly cited through references. All assistance and other publications associated with the work are noted in the front matter of this thesis. 
This thesis consists of five main contributing chapters. In the first we frame the issues relating to the second screen use case more thoroughly. Then, in the remaining four contributing chapters, we explore understanding and designing cross device attention. In chapters 4 and 5 we consider how we may better understand the ways users divide their attention between devices, towards designing more effective content and systems. Then, in chapters 5, 6 and 7 we consider how one may design user attention by intervening in the way they distribute their attention, exploring the autonomy of cross-device focus each method affords them.

The contributions of this thesis include empirical investigations of current phenomena, implementation and validation of novel technological interventions, new methodological insight, and detailed design guidelines from empirical studies.

We first outline the research agenda of this thesis by considering the related work in Chapter 2. We explore the initial concepts to inform the reader and situate the work, outlining some of the current open questions. Then, we, in Chapter 3, describe and justify the methodology undertaken in this thesis, framed with the key research questions we investigate.

In Chapter 4, we extend previous studies of attention by exploring users' current experiences of managing their attention across multiple screens through a largescale online questionnaire, then frame our findings, by exploring these phenomena through exploratory interviews with second screeners. The findings in this chapter allow us to outline the key drivers behind cross-device attention overload, from the perspective of users' reflections, and therefore make some broad, general conclusions with regards to the experiences of participants, and outline some po- 
tential guidelines from their comments. Our findings in particular give advice to broadcasters and application developers on designing for particular content types (i.e., genres), and provide a series of 'viewer types' around which to design their content, as evidenced by our extensive data collection and analysis.

In Chapter 5, motivated and shaped by the knowledge gained in the previous chapter, we conduct an extensive investigation into how changing the complexity of second screen materials affects the objective and subjective experiences of participants. We conduct an empirical study with participants in the presence of varying tablet complexity. By changing second screen complexity using standardised metrics, we present data to support the effective design of textual and graphical content for cross-device media.

Further, we also explore the subjective effect of the interaction of multiple information streams (i.e. the television and the tablet), and by doing so homing in on a set of heuristics for when best to present second screen content of a given complexity during a television programme. These heuristics, we suggest, can be used to allow designers to better consider the effect of certain television materials, and give some broad guidelines with regards to the appropriate textual and graphical complexity of second screen material.

In Chapter 6 we explore how the aforementioned heuristics of complex television can be used to control for dual-screen complexity. We explore, though the adaption of complexity on a second screen, the efficacy of 'curating' second screen complexity to the television material, based around the heuristics in the previous study. We conduct an empirical study of complexity curation by comparing this to a randomised baseline, and a case in which the participants can adapt the com- 
plexity themselves. By curating the complexity to our heuristics, we determine the efficacy of both our heuristics in general, and of the effect of curating materials compared to allowing users to manually adapt the complexity of the material. Then, by comparing this to the case in which the users can adapt the complexity of the material, we shed light on the effect of giving the users the autonomy of their own attention management.

In Chapter 7 we consider in more depth the control a user has of their attention, and how we can affect it in a cross-device media scenario, by giving the creator of the content a set of tools to mediate attention between devices. We conduct an empirical study to determine the effect of embedding various stimuli in a television programme, or in a tablet experience. We outline in this chapter how different methods of embedding stimuli in an experience, such as audio and visual notifications, can be implemented as design levers by practitioners towards the desired attention distribution, from an objective and a subjective perspective. We provide guidelines for initiating quick attendance, as opposed to methods in which the users may want to defer their attendance to the unattended screen.

In Chapter 8 we explore display commonalities - the mirroring of one content stream (e.g., TV material or second screen content) within the other. We evaluate this design space with professional broadcast practitioners, and then conduct an empirical investigation to determine the impact of the most successful methods towards understanding their impact, and designing towards positive UX with multi-device scenarios. Our findings invoke developers of cross device media to consider using such techniques, and give them design guidelines to do so for companion content, and a free browsing of the web context. 
To finalise the thesis, in Chapter 9 we outline some general discussions of the thesis, outline the limitations of the thesis and conclude by discussing how our work can be extended and embellished as the technologies and knowledge in this area grows. 



\section{CHAPTER TWO}

\section{Background}

In this chapter we consider the key work in the area so that we may better situate our research, which seeks to more completely understand, and enhance the design of, technology for cross-device television experiences. It is important to understand the origins of the second screen use case, so that we may better explore how it came about, and what it affords users. We first consider the origins of multidevice contexts, refining our scope to consider the recent emergence of second screening. By framing our scenario, and differentiating it from other cross-device and cross-screen scenarios, we then consider how mobile devices have dramatically transformed the television watching experience.

Taking a step back, we then consider the environments in which these interactions take place, and the potential technological enablers behind this - looking at the most recent developments for cross-device experiences. We then discuss what these technologies allow in way of 'intentional' second screen experiences. By discussing currently available publicly distributed applications, and those developed as research probes we then examine the existing research.

\subsection{MULTI-DISPLAY CONTEXTS}

Multi-monitor computing originates from a need to increase display space to view more information. Early multi-display systems were devised to concurrently view detailed pieces of information. Patents from the late 1970s suggest that engineers and scientists required more display space to view their data $[15,34]$, and therefore 
sought to increase their screen real estate by supplementing their cathode ray tube (CRT) displays with additional CRTs by their side.

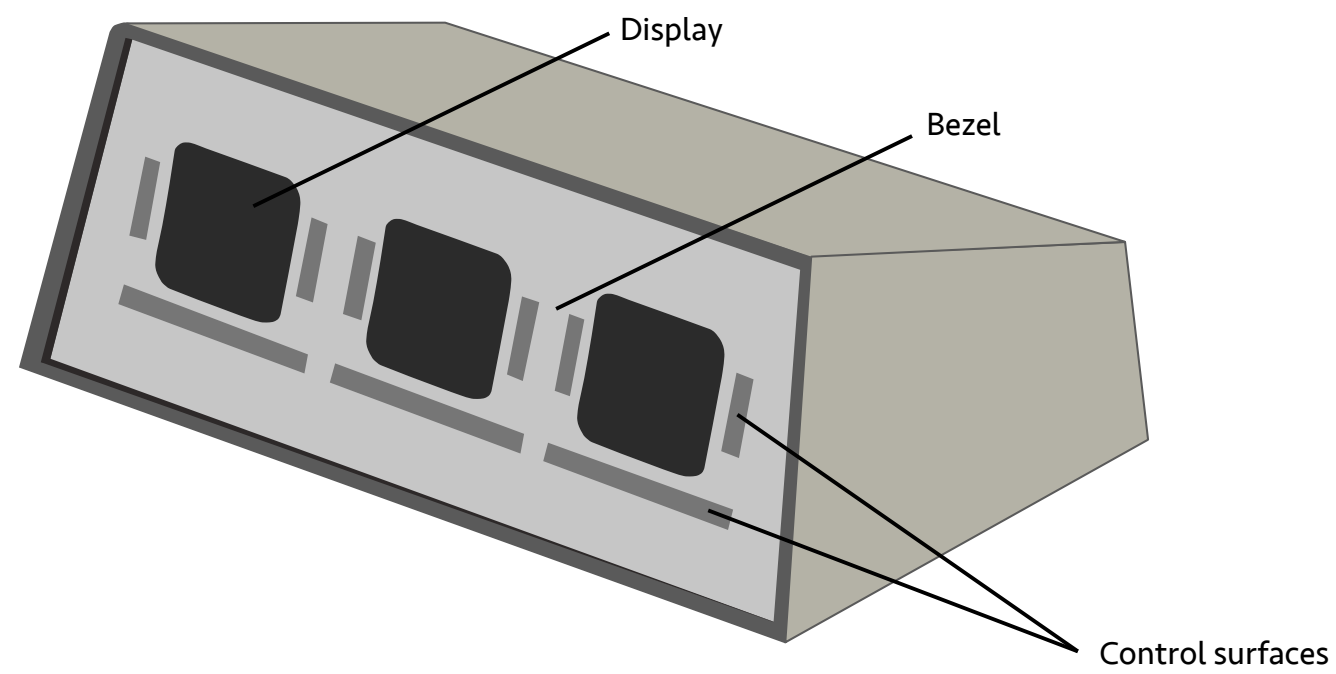

Figure 2: When requiring additional screen space, an obvious solution for scientists was to find ways of logically extending their current systems. Therefore, instead of simply making higher resolution monitors, they added additional screens to their workspace. The advantage of this is the fact that multiple instances of a screen allows us to better partition between different sets of data. Such workstations were typically bound by the limitations of the time - for example, the large bezels between the displays. Image replicated from associated patent [34].

Much human factors work has explored the effect of monitoring multiple displays in control tasks, for example, in stock trading [62], nuclear power plant control rooms[13] and air traffic control centres [88]. Researchers have extensively studied how air traffic controllers (ATCs) engage with multi-screen contexts to better manage their workload, towards optimal design of the display space for such a highconcentration and time-critical task [88, 99]. Lokhande and Reynolds [88] study the gaze of ATCs, exploring the way they switch their focus between the flight data manager, the tower information system and the camera monitoring the runway. 
This division of attention between screens and tasks is also explored extensively in non work-focused contexts. Karsenti and Fievez consider the impact of the tablet computer on the classroom [76]. In a study with 6000 children in the classroom they consider the impact of the tablet computer on learning in the classroom. While their key recommendations outline many positives of the tablets on the learning experience, they also allude to many potential risks around the distractions such an information-rich device introduces. Their findings are in accord with results from a 2013 study by Risko et al. [118], which surface the increased cost of engaging with non-lecture related materials on a device, in terms of attention and retention of lecture material. Such observations are akin to focusing on a mobile while driving. Driving and engaging with a device has been shown to dramatically slow reactions, reduce awareness and make drivers much more inclined to take risks [39].

Over time, as computing capabilities have risen, and costs have fallen, multi-display contexts have become ubiquitous in information-rich, office-work contexts. As discussed by Grudin [57], such uses of display arrangement allow us to divide our screen real-estate with the physical bezels around the two screens separating them in the middle,allowing us to split our work across screens in a multitude of ways. Grudin's study found that additional monitors are normally used for secondary activities, and for peripheral awareness of content which is not the main focus of the user. For instance, one of Grudin's participants notes that instead of clicking a mouse to cycle through windows to view a calendar, one can simply keep a calendar visible and instantly accessible in peripheral vision [57].

Most vitally, Grudin alludes to the way that second monitors are not generally viewed as just 'additional space', but a partition to physically distribute work across 
and take advantage of our rich $3 \mathrm{D}$ spatial cognition capabilities; providing more added value than a singular, larger screen would.

\subsubsection{Peripheral and Ambient Displays}

We may use a 'secondary monitor' to support more passive secondary tasks, and a 'primary monitor' for focusing on the specific task at hand, for example. Work looking at how we can use a secondary display to monitor a background task has been explored extensively in the HCI literature.

Most of this work focuses around monitoring of tasks, for example through changes in ambient lighting (as in the work of Matthews et al. [94]), and by providing peripheral information to a task through simple graphical scenes on a small peripheral display (c.f. the InfoCanvas project [102]). Some early work by Wisneski et al. [148] also looked into how an environment may be enhanced by assimilating ambient, information-rich displays into our environment.

Multi-display contexts have now extended from the more practical, productivityfocused endeavours, to media and arts to provide experiences. Utilising the physical partition between the digital worlds, and the increasing computing power and interaction capabilities of mobile devices, to enhance realism, control, and to foster a more tangible sense of space. However, though the bezels may have shrunk in these living room entertainment contexts, the distance between them has increased, creating two separate digital bubbles. Before considering this scenario deeper, however, we take a step back to consider multi-device computing, and the origins of multi-screen TV to evaluate the drivers behind such cross-device media contexts. 


\subsubsection{Multi-Device Computing}

Related to, but distinct from multi-screen environments, are multi-device systems, which involve computing experiences that span two or more devices. The focus of this thesis - multi-device media - is an clear manifestation of the latter. The ultimate goal of multi-device computing is in step with Weiser's 1991 vision of ubiquitous computing [144], wherein computers cease to act as single entities, but as one:

"Prototype tabs, pads and boards are just the beginning of ubiquitous computing. The real power of the concept emerges from the interaction of all of them.” [145]

However, there is no device which allows us to do everything effectively - we normally rely of the affordances of certain devices for given tasks. For instance, in a field study of multi-device workflows, Santosa and Wigdor [124] found that devices' form factors and capabilities are exploited in suitable tasks, for example many of their study participants used their mobile to monitor emails, switching to their mail client on their personal computer when a call to action was initiated.

Such diverse usage introduces some interesting challenges around how we can create unified experiences with this multitude of devices. Dearman and Pierce's 2008 paper "It's on my other computer!" [35] makes evident the problems around multi-device computing, mostly around continuity between tasks on devices, the roles assigned to each device and sharing information between their devices. More recently, since the explosion of capacitive touch-screen mobile devices this has become an increasingly important domain of research, with Sheridan et al. [127] 
arguing that the smartphone is now indeed the ubiquitous input device. With the increase in computing power and the dawn of cloud computing, many cross-device use cases are now being supported. Apple, for instance, offer Continuity ${ }^{1}$ - a service which allows all major services on one's devices to be integrated, for example to be notified and reply to a message on both mobile device and desktop.

Towards unifying cross-device experiences, much HCI research of late has focused on device-agnostic, multi-device applications. Pearson et al., for instance, express the importance of cross-device consistency in their work on collaborative reading of documents [111]. Such design principles are reflected in many popular collaborative document applications, such as Google Docs ${ }^{2}$, which allows users to share and edit documents in real time. In terms of providing cross-device interfaces, much recent work has more focused on using web technologies to distribute UIs across multiple screens [149], and explore methods to afford the user(s) the possibility of sharing real-time dynamic media across many devices [81].

\subsubsection{Origins of Multi-Screen TV}

Before discussing the work on second screening, we a step back from technology and consider the origins of the second screen case: what preceded it; the fundamental behaviours it supports; and, how it has, in a few short years, become one of the most common multi-device use cases.

It could be argued that second screen use case is a byproduct of our connected, mobile digital world. Our enthusiasm to interact with the digital while watching television is an increasingly prevalent behaviour that is transforming modern media.

1 Apple's Continuity: http://www.apple.com/uk/macos/continuity/

2 Google Docs: https://www.google.co.uk/docs/about/ 
We are passive in the television experience; it requires little for us to engage with the content, and it demands little from us. Compared to reading, for example, it requires little cognitive effort [12]. To read we must investigate line by line, interpret the semantic information, conceptualise its meaning, and attach this to a mental audio-visual narrative. With television, there are therefore often lulls in attention, the opportunity to freely interact with our mobile devices - searching related information, or engaging with information to keep ourselves stimulated.

This is not to say that the notion of multi-tasking while watching TV is a modern concept - it most certainly is not. Before digital ubiquity, we filled the lulls in attention with other side-activities. Previous to this use case attention was divided in other ways - as noted by Schmitt et al. in 2003 [125], who monitored 50 individuals over 10 days with cameras, finding that television viewing was regularly accompanied by eating, reading, or social interaction.

Before the digital age, many behaviours indicated a need to support television with additional content. One could argue that pull-outs in magazines, or annuals oriented around TV programmes were an early case of companion content to enrich the experience. Similarly, television and radio audiences have been connecting with broadcasters since the 1970 s through letters, phone-ins, and even real-time interactive games such as The Golden Shot ${ }^{3}$. This increasing desire for two-way interaction between audience and broadcasters has manifested in the digital, and is closer than ever to being realised.

3 Example of The Golden Shot on YouTube: https://goo.g1/UTVOAJ 


\subsubsection{Second Screening}

Though there are many other cases where second screens complement another leisure activity (for example, in the world of video games [25, 40]), TV is the primary focus of the HCI community, and many ethnographic studies document just how nuanced and far-reaching this use case is. This research has most commonly taken the form of in-situ studies of second screeners in their homes. Before the smartphone, in studies such as that by Bernhaupt et al. [14], it was noted that users regularly interact with mobile devices as a foreground or background activity while watching television. Further, Tsekleves et al. [134] saw, in a study with 27 families, a strong desirability for the integration of secondary devices and services into interactive television services that are specialised to specific online activities such as social networks and email.

The proliferation of internet-equipped, interactive devices into our livingroom has, to some extent, filled the lulls in attention and our need to interact with content providers. A 2014 report from OFCOM [107] (the UK's communications regulator) suggests that second screen interactions are skyrocketing universally, across all demographics. There is vast usage of personal devices in the living room, and a rich set of literature around the HCI for TV community is trying to catch up with these trends and better understand this use case. D'heer et al. [37], for example, look at how we consume media on second screens in the everyday context. Further, Holz et al. [67] set out to understand this behaviour on a minute-by-minute level to gather a more thorough impression of users' motivations for device use while watching TV. By studying the usage of seven families, they were able to infer that the majority of second screen activities are unrelated to the programme at hand only switching focus to the television at key moments. In addition to this work by 
Holz et al., Vanattenhoven and Geerts [138] further explore how people use secondscreens in front of the television in the home. They worked with 12 households to record media usage and communication behaviour while watching TV or video. In general, they found that the majority of the reported second-screen uses were not related to the broadcast because viewers lost interest in the program. They propose, to remedy this, that we may be able to use second screen applications to seize such moments to regain viewers' attention by providing additional social media, programme-related material, or further recommendations [138].

Finally, Courtois et al. [31] note a divergence of use in second screeners - those who focus only on the television; those who combine television with other media (e.g., laptop, tablet, or print media). They note that, at least in 2012, whilst second screening is rife for tangential browsing, the potential of second screen apps is heavily under-utilised, findings also reported more recently (2015) by Holz et al. [67].

Over the literature two prominent viewing patterns become apparent: second screen browsing which is incidental - i.e. users would be doing it regardless of the television's presence; and that which is a direct consequence of their television viewing, for example, someone searching for an actor they see in a programme - frequently termed complementary [54,80] second screening. In this thesis, we focus predominantly on the complementary contexts as these are the situations in which the users wish to engage with both screens, and indeed the ones the academia and industry aspire to develop new multi-device experiences for. 
The notion of the fully connected home is making the vision of this exciting multiplatform content more realisable. Given that recent reports are indicating that internet-enabled TVs are now the norm (according to a 2015 survey, internet-enabled TV penetration is $>50 \%$ in the US [55]), and most are connected to a home network, the infrastructure is mostly in place. Therefore, recent work has looked into how we may integrate such systems into the norm for users - working on creating standards for content creators to fulfil. There are three key factors when considering cross-device infrastructure and systems for multi-screen experiences, which we will now discuss.

Firstly, there is latency - to what extent the content across the devices synchronises across the devices. This is a key area of concern for many multi-device experiences that require temporal synchronisation. For example, with subtitles, it is vital that the information is synchronised in the order of less than a second [121] and for experiences with intercrossing visuals and audio even tighter synchronisation is required (c.f. the synchronised director's commentary in [70]). Moreover, low-latency systems are imperative for collocated devices [53], and remote shared viewing [52]. There are many factors that affect inter-device synchrony, most notably the quality of the internet and local network connections of the user.

Secondly there is the setup cost - the amount a user has to work to setup the experience. This depends largely on whether they need to download specialist apps, own certain hardware, or need some prior technical knowledge to initiate the multi-device experience. 
Finally, there is the mutability of the content to the device - what devices the content can adapt to. These are dictated by internet connection and the method for data distribution. A lot of content nowadays is responsive - such platforms offer great flexibility in terms of device capabilities and screen size.

Cross network standards, such as the BBC's Universal Control API [9] are not yet a norm. However, this API allows for the control of set-top boxes and similar devices on a given network, and for all devices on a network to communicate. The BBC envision that this will enable multi-device orchestrated experiences, and enhanced interaction potential for viewers [83]. Such standards not only allow for interfacing for numerous Internet-of-Things (IoT) devices but also has strong implications for the design of custom interfaces and integrated controllers, with clear benefits for accessibility.

Recent work by Vinayagamoorthy et al. [141] proposes an open communication standard between Internet-connected TVs and companion screens, over the home network. This work provides a standardised way to enable this synchronisation between the TV and any personal device on the home network, which is frameaccurate. Such work, along with work such as that by Zorrilla et al. [151], which also looks at a method for synchronous cross-device experiences, may allow for seamless simultaneous multi-screen video experiences, such as alternate angles of live footag, with no perception of lag in the near future.

To achieve low setup costs, a great diversity of connection methods have emerged. For example, audio watermarking (also known as audio fingerprinting) has been used by a number of applications to temporally synchronise the device and the television (e.g. in [67]). Audio watermarking overcomes the complications of client- 
server architecture by simply embedding imperceivable high frequency $(>20 \mathrm{kHz})$ audio cues in a piece of video material. These markers serve as triggers to cue content on the device, resulting in reasonable synchrony between devices.

Audio watermarking, however, has major shortcomings - most notably inconsistencies between devices, poor handling of user interactions, and its proprietary nature [36]. It has therefore not been widely adopted. Parallel to this, much work has looked at standards for multi-stream, multi-device media synchronisation (see [36] for more detail). Due to international standards, such as hbbTV (Hybrid Broadcast Broadband $\mathrm{TV}^{4}$ ), networked synchrony is improving vastly for collocated and remote shared viewing. This noted, technologies which allow for frame-accurate synchrony between a device and television are very much still at the research stage - for example the promising open communication standard proposed by BBC Research and Development [141]. As such technologies become more widely adopted by broadcasters and developers - driven by a need to provide an experience for their users - it is likely that such networked experiences offer a strong future to cross-device experiences.

\subsubsection{Object-Based Broadcasting}

Since its inception, TV programmes have been inherently linear and one-way. Essentially, the materials are made in the studio - the video, audio, and additional assets are produced, and then are mixed together into a finalised programme and broadcast as singular linear media items for users to receive at a set time on their television sets. Conversely, object-based broadcasting, flips this notion on its head. OBB extends the now ubiquitous concept of 'watch on demand' by allowing each 
individual to have a totally unique experience. The process entails gathering each piece of media, but instead of sending this as an immutable block as before, each piece is sent individually via the internet. By sending the media elements separately, it delegates the construction of the material to the 'other end'. Once received the programme can be assembled to the user's needs.

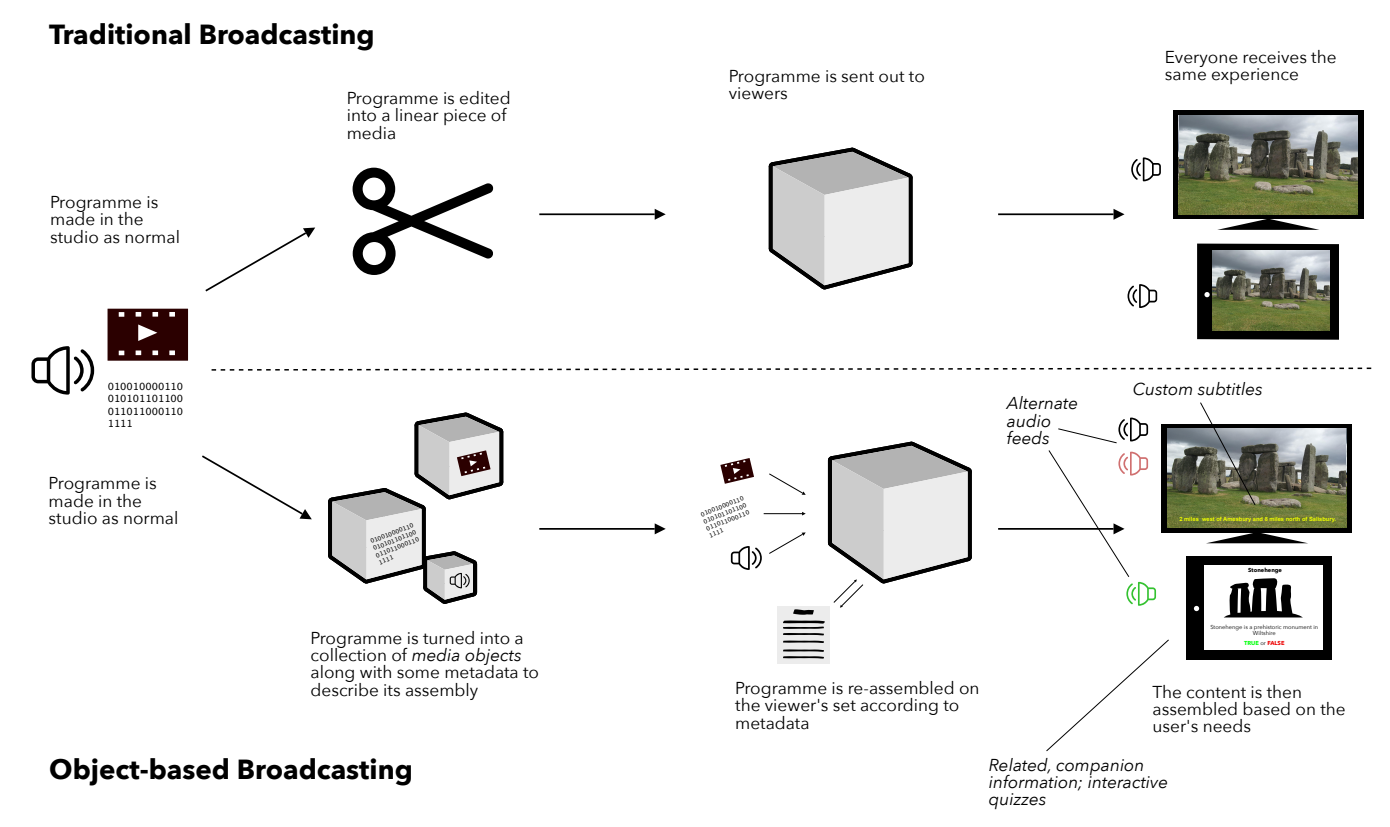

Figure 3: Object-based broadcasting paradigm, adapted from [91].

By providing content as objects, with associated metadata, we empower users and developers to encompass a variety of needs. We can not only adapt systems to cater for different technological setups or for those with physical disabilities [9], but we can also craft new and exciting media experiences - for example we can adapt the audio to a football match to the side of our choice [91], or by providing 
responsive [48] renditions of stories - intermixing a story with details about the listener, by getting information from their social media feeds or by analysing the user's browser data [133]. OBB manifests in varying levels of responsiveness [6], some simple and already realised, and some ambitious and still within the domain of research. Regardless, the level of adaptability, OBB and similar concepts suggested by academia and industry, through increasingly connected homes, are a likely catalyst for cross-device experiences on the home network.

\subsubsection{The Increasing Ambiguity of the Second Screen}

This thesis focuses on mobile devices, the most ubiquitous manifestation of a second screen. However, if we can provide content agnostic to device, we can envision exciting new experiences across a myriad of internet-connected screens and devices - something academia and industry has recently begun to consider. We, therefore, briefly consider what a screen can be.

'Second screening' [10,41, 66], 'dual-screening' [23], 'multi-screening' [140], or even 'many-screening' [4] have become popular terms in the HCI for TV community. However, for the purpose of this thesis, a significant point of discussion is what constitutes the second screen. In general, when using the term 'second screen' we normally refer to a tablet computer or other type of mobile device such as a smartphone. However, we could argue that any display in which we present visual information alongside some kind of primary screen can be considered a secondary screen - indeed, including the TV itself. Extensions of the screen [58, 139] through projecting outside of the TV, extending the entire screen to the wallpaper [65] are examples of how we are constantly trying to 'think outside the screen'. 
If we are to take a step back and consider the rich continuum of a what can be considered a 'display', we could be referring to anything from a flat tablet screen, a deformable UI [120], an ambient Orb [94], a projection [71], or even a mixed reality physical entity in the room - such as a toy Dalek ${ }^{5}$ which move synchronised to on-screen Daleks around a user's living room during viewing [70].

We consider dual-screen experiences as contexts in which the devices additional to the TV are either user driven, or promoted by a broadcaster. User-driven behaviours are typically associated with someone engaging with things they see and hear in a programme, e.g., by searching terms or following a debate on Twitter. When presented by a broadcaster, such experiences are focused on supporting this behaviour, for example by providing content on a dedicated 'companion' application which presents related information, or a social media feed for the programme the user is watching. We now discuss how second screen behaviours have manifested in the creation of designed experiences such as companion applications; reflecting on their adoption; explore a categorisation of the applications, and then progress to see how researchers have aimed to push forward the design space with empirical studies and prototype systems.

\subsubsection{Publicly Available Applications}

Second screening is now an everyday part of the television landscape - we follow hashtags along with live programmes and discuss events in real time (the extent of this 'live' effect can be seen in [87]). Social media is especially ingrained in sports 
and live debate, to the extent where it can affect its outcome [93]. Social media campaigns have also been shown to significantly improve brand awareness during major events such as the Superbowl [64]. To provide insight into the current state of the art in publicly distributed applications we will now describe some of the most prominent second screen applications for television programmes created by broadcasters and independent application developers.

Initially, such applications began as small rollouts to test the feasibility of the infrastructure, and the viewer's receptibility. For example, the BBC rolled out their Autumnwatch second screen application pilot. This was tested on around 400 members of the British public and blurred the lines between a research prototype companion app and a commercial deployment [8]. Since the Autumnwatch app, the BBC have experimented with many more applications. Examples include The Predictor [5] - a second screen application that allowed users to play along with The Apprentice programme and predict who would win; and an interactive play-along to accompany the Antiques Roadshow, in which the users had to guess - during the programme - how much particular artefacts were worth [146].

Other broadcasters have also explored companion applications to enhance the viewing experience. For example, Channel 4's Million Pound Drop Live application, in which users can play along with, and compare to, other contestants in the live programme [1], and a plethora of applications to create interactive, realtime experiences for major programmes such as Breaking Bad, and movies such as Avengers through AMC's StorySync [101]. Finally, major online media providers, such as Amazon Prime Video, support second screen content: the $X$-Ray [2] service provides encyclopaedic, time-synchronised, content and information about people featured in a film when a user interacts. 
With regards to widespread adoption, the growth in companion applications over the past few years has been steady and met with mixed reception. Though general browsing on a second screen is ubiquitous, second screen applications are not. In the aforementioned 2014 OFCOM report [107], OFCOM suggested that general uptake in companion applications is still relatively low in the UK - for example, one of the most popular programmes with a second screen app (X Factor) saw 547,500 downloads of its offering, which constitutes $5 \%$ of those who watch the programme. This figure, however, doubled from 2013 and appears to be growing year on year - as of July 2016, there are somewhere between one and five million downloads on the Google Play store alone. The report's data indicates that the most successful applications (in the UK at least) appear to be those which offer social aspects, for example, the 'TV Guide' app constitutes about $10 \%$ of UK viewers. As of 2014, Remote control apps for televisions tended to see relatively high, but nonmajority uptake. For example, Panasonic's Viera remote saw an uptake of about $25 \%$ of those who bought a Panasonic TV.

Though there is clearly a demand, and some reasonable growth in the usage of companion applications, there are a few major barriers to use. One such barrier is the effort required to set up the dual-screen experience. Often, users must visit a specific link or download an application, and rely on synchronisation technologies such as WiFi and audio watermarking. This setup cost can be likened to playing a board game; the enjoyment of the experience must outweigh the perceived setup cost, otherwise nobody will play. 


\subsubsection{Commercial and Prototype Companions: A Categorisation}

Companion applications manifest in many forms to support our pre-existing behaviours. With this in mind, we suggest that we can categorise them by the behaviours that they support. We divide companion applications into the following types: supporting tangential browsing; complementary social media; and as a method of extending the remote control.

There are numerous applications to support users when searching materials related to a programme. These either support passive information presentation to the user, in which complementary content is provided at timed intervals, or support more interactive information search, for example through tree visualisations [38]. This information is typically trivia related to the programme. This can be a series of slides with pictures and short blurbs to support the programme, for example, BBC's Autumnwatch deployment, which provided time-relevant information - typically simple graphics and text about the programme on a second screen. Such experiences can also be more interactive, for example, a companion app made for Channel 4's multi-platform campaign "Foxes Live: Wild in the City" [30]. The app, which ran alongside the programme, provided the user with real-time information about wild foxes as they viewed them on live TV.

In addition to entertaining, these applications also have the potential to educate. Fallahkhair et al. [44] describe the potential for supporting language learning using a companion application. They describe an approach that suggests presenting supplementary translations on a mobile device may aid in learning a language by clarifying terms in another language in English. They note a significant benefit the 
second screen brings - content can be individual not distracting to other viewers of the same experience.

Many applications aim to embellish the second screen experience by providing additional information that allows a user to get a better gist of the programme, and its characters as a whole. Those which aid the understanding of complex concepts in a programme, or provide additional information to the programme aid in 'world building' are a form of transmedia 6 . Murray et al. [105], for instance developed a companion application to support users understanding complex long form television narratives. 'Story-Map', allowed for viewers to contextualise characters in relation to each other by making a map that described their ontology, allowing program viewers to provide some degree of backstory and a gist of the character relationships without spoilers. Similarly, Dowell et al. [38] augmented an information-rich programme via an interactive companion application. In this case they explore how information in an astronomy documentary can be summarised through interactive concept maps. In addition, Eversman et al. [41] explored how glance-able pieces of information can be introduced at key moments in a programme to link together Marvel's United Universe.

Television gives us a common talking point and brings together families in their living room. Therefore, it is unsurprising that applications have been developed with an aim to support social viewing, as well as remote interactions through social media. We turn now to discuss how companion applications have been employed to support social viewing.

6 The notion of spreading a narrative across multiple platforms or devices, for example, a movie which is also a part of a comic book universe 
The earliest indication of work which supports the sociality of television is work done by Microsoft - in 2004 Regan and Todd [115] explored how we may integrate online social functionality (instant messaging) into a media centre. Such early interactions, however, have been restricted to a single screen. The second screen is now an everyday bit of the television landscape - we follow hashtags along with live programmes and discuss events in real time (the extent of this 'live' effect can be seen in [87]). Social media such as Twitter is especially ingrained in sports and live debate - to the extent where it can affect its outcome [93], and social media campaigns have also been shown to significantly improve advertising during major events such as the Superbowl [64].

More recently, numerous academics have then made the logical step to providing second screen interact to television experiences. Much work around this was influenced by Geerts et al. [50] who looked at the implications of genre on social TV platforms - essentially finding that news, soap, quiz and sport are genres during which our participants talk most while watching and are thus suitable for synchronous social interactive television systems. Moreover, in [51] Geerts et al. from a culmination of studies consider a series of heuristics by which designers of social TV systems may better design sociality.

Second screening and time-shifted broadcast have become so popular, much work has explored how we can deal with spoilers - clearly, when we view programmes out of sync with their respective social media feeds there will be discontinuities. Basapur et al. [11] while trailing their companion app, FANFEEDS (a companion content authoring app with a social element), they noted several instances of spoilers - for example football scores being revealed in the social media feed by one participant before the other had seen the game. Issues with discontinuities such 
as this between screens have inspired people to look into automatically detecting spoilers in programmes (c.f. the work of Boyd-Graber et al. [19]), and even looking at detecting exactly what programme a user is referring to online [32]. Further to work by Basapur et al. [10, 11], several academics have looked at how we may lower the burden of dealing with social media feeds and supported browsing over many apps by integrating them into a single application. For example, Hess et al. [63] consider a concept for unifying social media feeds.

Turning to co-located experiences with mobile devices while watching television, McGill et al. have looked at how we may better design for shared experiences. They looked at how we can promote the sharing of personal content through screen mirroring $[96,97]$. Their work focused on extending the private world of the second screen to incorporate multiple viewers towards equal participation and improved awareness in multi-user multi-display contexts. This work utilises the sociability of TV and extends the notion of families and friends gathering around their television sets to unwind - using television as a vehicle for conversation and 'real world' social interaction.

In a similar area of endeavour Anstead et al. [4] look at the effects of many-screen viewing on a companion application they developed to allow users to revisit highlights from the 2012 Olympics. Beginning with with one shared tablet, they gradually introduced new devices to see the effect of introducing new media to the users, finding that additional devices facilitated more personalisation and autonomy in their second screen viewing. 


\subsubsection{Extending the Remote Control}

We turn now to discuss work which utilises the ubiquity and mutability of touch screens towards providing more customisable and effective controls for television. Irrespective of the technological developments over the past 20 years, the television remote still sits at the centre of our interaction with televisions. They are cumbersome, inconsistent and bound by their physical limitations, as discussed in more detail in Bernhaupt et al.'s 2008 ethnographic study of living room trends [14]. There have been a number of tablet applications released that allow for the control of a television with a tablet or smartphone. As previously mentioned, work in this area and the ubiquitous adoption of smart devices has culminated in manufacturers creating second screen applications which act as remotes or EPGs (Electronic Programme Guides) - the LG TV Remote; the Samsung Smart View; and the Sony TV Sideview to name a few. We now briefly touch on academic literature to gain insight into the work done, and how we may design for such control-based interfaces.

Work by Cruikshank et al. [33] explored what a customisable remote, such as a second screen PDA, can bring to the world of interactive television. They describe a solution that removes the interactive element from the television (or its remote) and transfers it to the second screen (PDA). In doing this, the authors noted a " $d r a-$ matic improvement for effective interaction and navigation for iTV interfaces and services". This delegation of interaction to a more suited device appears to not only allow improved interaction but allows designers to save space on the main display to do what it does best: displaying. Further, Bobeth et al. [17] studied methods for controlling a television app, comparing two alternatives to the remote control; air gestures, and a tablet computer interface. Results implied that mirroring the televi- 
sion on a secondary device and allowing the users to interact with this provided a more intuitive experience than the remote and the in-air gestures, likely due to the familiar nature of the device, and the direct manipulation capabilities. Again, allowing interaction to be undertaken by the secondary device was shown to be significantly beneficial. Interestingly, their study also showed that older users, who are typically less familiar with tablet computers, adapted to the concept of controlling the television adapted quickly. EPGs are a cornerstone around which other second screen functionality can be added on - for example, You et al. [150] explored the concept of designing a multi-screen EPG which linked other users in a similar style to a social network.

Though it is clear that second screen applications allow designers to leverage greater interaction benefits than remotes, the real impact is made when we consider the needs of those who require some degree of customisation for the device. For instance, Barrett et al. [9, 70], introduce the Universal Control API, which allows any configuration of the interface to be developed, and for external sensor technologies to be used, to cater for the needs of specific individuals.

\subsubsection{Authorship, Sustainability and Artistic License}

A significant question in the area of companion content is "where does the content come from?". The limitations and opportunities for creating sustainable applications are surfaced by Messina et al. [98], whose work looks at developing an underlying architecture for efficient production of second screen applications for broadcasters and media companies - for example, tools for authoring companion experiences. It is evident that the content can be broadcaster created (which is timeconsuming), or driven by some algorithmic or user-generative approach. 
The most common instance of companion content is broadcasters generating second screen content to be distributed to their audience - a notion explored in depth by Geerts et al. [53]. Examples of synchronised content are the aforementioned $\mathrm{Au}-$ tumnwatch experiment, and the Britain's Got Talent companion app [112]. Problematically, such approaches are labour intensive and additional work is needed to create the companion content. A potential solution to this is to develop algorithms to take information from the internet at specific points in a programme - an extension to personal casting, or the notion of providing broadcast customised to users' interests [77, 95]. Algorithmic implementations of content retrieval for second screens may use techniques such as data mining to infer complementary content for users from the web, based on their viewing habits. As discussed in detail by Morales et al. [103].

One could argue that the first approach;edited by a human and produced with the programme in mind is likely to offer a more coherent experience, with the more artistic human touch that such a scenario can afford. One possible solution to this, proposed by Basapur et al. [11] outlines a human-driven companion content generating experience. Their system 'FANFEEDS' uses social networks to choose content from the web that is appropriate. They propose a point-based system in which users can become 'gurus' of generating content, and therefore be more trusted to provide content that is both relevant and interesting.

Ultimately, it is up to the content providers to decide how companion content is created. On one hand, we could have artistically created companion content, designed and edited in tandem with the television production, to embellish the experience. And on the other, one could envision a totally algorithmic system which pulls content from the internet. A programme in which a broadcasting corpora- 
tion take a lot of pride in creating may be conducive to more orchestrated content, whereas lower budget productions may have their companion content authored algorithmically.

Though second screening is now ubiquitous, uptake, and the development of, crossdevice media is still in its infancy. In terms of adoption, such systems are still bound by several factors, some of which are in the research community's control. As we considered in the ethnographic research, the second screening is a mixture of related and unrelated interactions. The related, which are of interest to the present thesis, are often done on the users' familiar applications - their web browsers or their social networks. Now, considering factors more within the research community's control - it is evident that there is much to be done in terms of the technical infrastructure. As discussed in this chapter, there are a great number of methods by which multi-device experiences can be done, but no standard way to do so. The field - especially in HCI research - is disparate and largely uses proprietary, or Wizard of $\mathrm{Oz}$ approaches to inter-device experiences. To further innovation, the research community should aim to converge on a more standard approach. Effective, open source standards are likely to be a strong focus for this.

On human factors in the multi-device use case, there is much to be considered in terms of how we design for the attention and the requirements of our users. Future work should consider at each step of development the design of the scenario with the users in the loop - empirical studies should be run both in a lab setting and deployed longitudinally before en masse distribution. We should build models of how users manage their interests and attention by seeking insight from previous 
human factors research, and develop models and interventions of our own. And, vitally, as with all research it is up to those who develop the systems to heed it strong feedback loops between industry, broadcasters, and researchers are therefore vital to the success of this field.

\subsection{CONCLUSION}

In this chapter we have covered the state of the art in cross-device media - an exciting, fast growing and potentially very rewarding area of endeavour. We have explored the concept of users freely interacting with mobile devices, and considered how the cross-device television scenario compares to other multi-display contexts. By probing the current trends and behaviours we are seeing from industry and academia - from both an infrastructural, design, and a research perspective - we then further considered the work of those who wish to enhance the UX of such contexts.

Regarding the focus of this thesis, we have started to consider some of the physiological bottlenecks of this divided attention use case. In the remainder of this thesis we aim to better understand these phenomena, and design interventions to improve the UX of the connected living room. Before considering the bottlenecks of the use case further, however, we first describe the methodology of this thesis so that we may properly outline the key research questions and the scope of the project. 


\section{CHAPTER THREE}

\section{Methodology}

The focus of this thesis is to understand the effects of the second screen use case on users in terms of attention and to consider how we may improve their design. To quantify the empirical work in this thesis, this chapter describes the research objective and questions so that they may be explicitly considered and referred to throughout the thesis. In this chapter we give an overview of how we address the research questions in the thesis and outline our research methods, environments and scope, towards facilitating and contextualising the research undertaken in the remainder of this thesis.

\subsection{RESEARCH OBJECTIVE AND QUESTIONS}

The primary concern of this thesis is to consider additional impact a mobile device adds to the use case, and therefore more effectively design multi-device systems to account for this. The main research objective of this thesis, then, is to:

Gain insight into the way users manage their attention when engaging with dual-screen media and develop techniques to assist its management.

To explicitly outline the remainder of this thesis in terms of this research objective, we pose the following research questions (RQs):

RQ1 What are the attentional problems users face when viewing TV while also interacting with a mobile device? 
RQ2 How can we design optimal second screen content which accounts for the perceived complexity of dual-screen material?

RQ3 How can we effectively intervene in user attention to improve the way they manage it?

$\mathrm{RQ}_{4}$ To what extent should the user be in control of the way their attention is managed in a given context?

\subsection{RESEARCH OVERVIEW}

The research approach in this thesis is divided into two main areas - understanding and designing. RQ1 considers understanding. To understand the ways in which users engage manage their own attention when dual-screening we first, in Chapter 4 , outline the issues found in the literature and elicit information from users in a large scale questionnaire. From these reflections on attention management from users, we then, in Chapter 5 , probe deeper into understanding how the perceived complexity of material affects the users when engaging with second screen material while watching television. In learning more about the way users experience second screen complexity, in Chapters 5 and 6 we shed light on RQ2, learning more about the limitations of attention in terms of second screen visual complexity while watching television.

Towards fulfilling RQ3 we, in chapters 6, 7 and 8, explore methods by which we intervene in users' attention through a number of techniques. In chapter 6 we explicitly explore the tradeoff of giving the user the ability of adjusting the complexity on the device themselves (via a more/less information button) versus dynamically adapting it around a set of heuristics. 
This exploration of autonomy (RQ4) is further explored the next two chapters, first by attempting to totally removing user attention by telling them where to look and when via notifications (as in Chapter 7 ) and then by giving the user additional mirrored elements within their device towards allowing them to more effectively manage their attention themselves.

\subsubsection{Research Methods}

This research used mixed methods. Initially, to capture data around the users current behaviour with second screening we chose to use an online questionnaire, due to its large reach and their ability to capture subtle nuanced information. This allowed us to capture broad insight into the use case with a large corpus of qualitative data.

Following on from the capture of more broad usage data, we aimed to answer more fundamental questions by using lab studies with quantitive measures, framed by qualitative measure as we were testing specific hypotheses around the way the users managed their attention. To evaluate the efficacy of our interventions we used a mixture of quantitive (e.g., eye-gaze or on-device log data) and qualitative data capture, predominantly asking focused questions with Likert-based questionnaires, framed with semi-structured interview to allow us to contextualise our insights.

\subsubsection{Research Environment}

With regards to the research environments used, we predominantly used the BBC's usability lab, except for the study presented in Chapter 8, for which we used the us- 
ability lab at Swansea University. The BBC's lab is a purpose built environment designed to have the feel of a typical environment that technology users may second screen in - essentially a mock living room with soft furnishings which is used on a daily basis by the BBC to test their latest technologies. Similarly, at Swansea University, we utilised a mock-living room which is split between a living-room arrangement and a generic usability lab. All environments were isolated to extraneous noise and interference.

With regards to the participants used in our studies, we screened participants to be from the demographic of people who engage with mobile devices while watching television. They were recruited via incentives and were taken from participant pools at Swansea University, BBC (non-related) staff and members of the public via a $B B C$ recruitment agency. As we mostly selected from students and staff at the BBC and screened for second screeners, our sample over all studies of 362 participants was younger $(25.13$ years; $\mathrm{SD}=8.56$; $\max =65 ; \min =18)$ and more likely to be digital natives than the average UK population. However, given that the general demographics of second screeners is younger than the UK population ${ }^{1}$, this sample is a reasonable representation of second screeners.

Regarding the stimuli used in the lab studies, we predominantly studied 'designed' second screen content on the second screen. We did so such that we may have more control over its variability and more effectively make contrasts between our experimental variables. We also strictly controlled the television content the users watched during the studies so that this could be ruled out as an influence in the experiments.

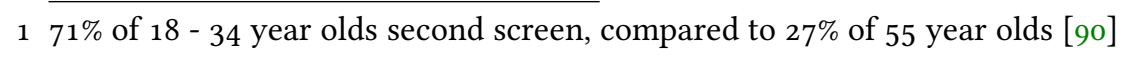




\subsubsection{Research Scope}

The main objective in this thesis is to gain insight into the dual screen use case and consider how we may design it more effectively. Therefore, the research presented in this thesis deals with two predominant types of study: firstly, in Chapter 4 , we conduct an online questionnaire; and secondly, we conduct the majority of the empirical work in this thesis via lab study. The scope of this research, then, does not explicitly explore our interventions in situ. This active decision to study our research questions in the lab was made because we wanted to capture detailed information in a controlled environment to investigate the research questions empirically. This controlled environment allowed for experimental controls to be investigated without experimental variability through external interference, which is typical of many studies of interactive television, outlined in the Background of this thesis (Chapter 2).

CONCLUSIONS

In this chapter we have outlined the main research objective and questions so that we may more accurately quantify our empirical work in respect to its goals. We also outline the analytic framework by which we explore the research questions in this thesis, noting our choice of research methods, environments and scope of the thesis. We now, in the next chapter, begin to understand the attention issues around second screening in more depth and continue to empirical studies to answer our research questions. 



\section{CHAPTER FOUR}

\section{Bottlenecks of Cross-Device Scenarios}

In this chapter we explore the issues faced by users when dividing their attention between multiple devices and screens. Currently, much literature indicates that TV with additional content can induce some degree of attention overload, no study properly explores this empirically. Most empirical studies around attention focus around eye-tracking in the lab, and do not gain an encompassing picture of attention in everyday viewing. Therefore, in this chapter we build on the work of the previous section by exploring the literature in this area. Then, towards gaining insight towards RQ1, we contribute to understanding this use case more completely by conducting two empirical studies which investigate the scenario from typical second screeners' perspectives. We first describe a study in which we conduct a large online survey with 260 participants to frame this use case quantitively. Then, progress to discuss the more nuanced details of the use case by exploring viewing habits with 20 second screeners through semi-structured interviews.

Today our visual attention is consumed by digital devices - a multitude of information streams compete for our attention in both the auditory and visual domain. Dual-screen attention is of significant concern to those who wish to design second screen experiences. Our attention is a finite resource, and designers of second 
screen applications have begun to consider how a user is likely to manage their attention. For video media, attention is a complex and nuanced phenomenon. As TV is multimodal, and greatly diverse in genre, it means that a reasonable baseline level of focus is required to fully engage with much material in a programme. As reported by Geerts et al. [50] in a 2008 paper on genre for social TV, genre itself clearly is highly impactful to the use case in general.

Now, with the introduction of a second screen, and with an increased number of user behaviours, such impacts will likely be exacerbated, and the effects on attention are likely to be significant. Such dual-screen scenarios typically involve us resting the device in our laps, or cradled in our hand in the periphery of our vision. Brown et al. [23] phrase this as the 'sit back' nature of the scenario. This likely hinders our ability to shift attendance to the secondary screen or to the TV, as we are required to significantly adjust our gaze or posture, adding increased switching cost (see the work of Rashid et al. on multi-screen switching cost [114]).

Second screening can be loosely described as a divided attention use case in which, over some time, the user divides their attention between two devices. However, it is clear that they switch their attention between the individual elements (in this case screens), sustaining their attention on one as the other becomes peripheral. Research suggests that users tend to switch their focus between tasks to compensate for the fact that one tends to make miss more information when attending to multiple tasks simultaneously (see [147] pg. 40).

Second screening, then, is perhaps analogous to driving a car while distracted. To drive safely, one must pay attention to the road at all times. The drifting of our visual attention while driving, for example to return a text, can lead to us missing 
vital moments. Cook and Jones, for example, show in the Journal of Injury Prevention [29] that engaging in texting and browsing the web while driving is not only distracting to the driver, but also leads to a substantial increase in crashes and citations.

Even with sustained visual attention to the 'primary task' of driving, the cognitive task of dealing with multiple competing perceptual streams (i.e., speaking to someone) has also been shown to be detrimental. Iqbal et al. [73], for example, investigated the role of cognitive dialogue on participants while driving a car, finding that sustained conversation, especially during cognitively involving driving exercises, was of particular challenge. This is, perhaps, analogous to the competing and combining streams of information between the television content (the road) and the mobile device (the secondary task), as shown in Figure 4.

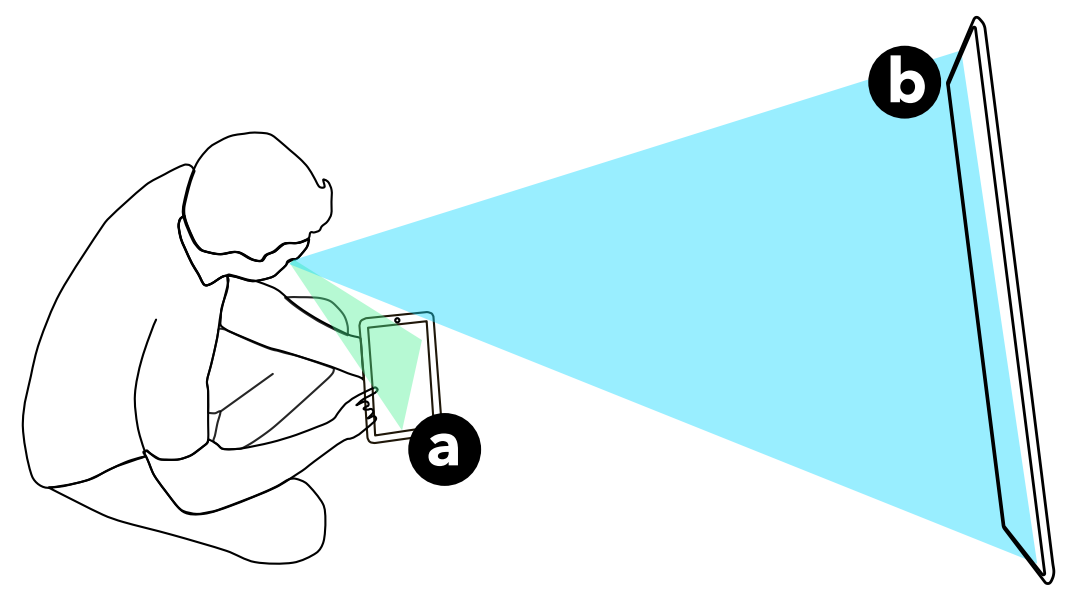

Figure 4: In cross-device scenarios users tend to switch attention, as opposed to divide. Typically, users rest the device on their lap, in their peripheral while engaging with the television (a)) and vice versa for when engaging with the tablet (b)). This creates a visual congruence between the foci, meaning that they must constantly check the device for new content when focusing on the TV, and monitor the auditory stream of the TV for points that pique their interest. 
In addition to the cognitive aspects of the scenario, the visual congruence between the visual foci leads to a great cognitive and visual disjunct. In addition, it is evident that users will have to engage with textual or visual content on the secondary device while monitoring the auditory feed of the television to engage with both screens - a task which is physiologically challenging as humans do not cope effectively with simultaneous comprehension of text while reading [123].

As shown in Figure 5 our visual focus is relatively small. The centre of focus only has a focus of $5^{\circ}$. In terms of visual perception, within this focus we can determine highly specific features, such as text. Eysenck and Keane [42] (pg. 151) liken this focused visual attention to a spotlight - everything within this small field can be seen with relative ease, however, outside of this, there are regions in which we can see progressively less as we move from the focus. In the near peripheral (approximately $5^{\circ}-30^{\circ}$ ) we capture less detail, and outside of this in the peripheral vision (up to $60^{\circ}$ ) we can only we can percieve only simple features - for example, colour. Further, due to the way that our visual senses have been shaped by evolution, motion - especially horizontal [43] - is a major factor in attracting visual attention ${ }^{1}$. Such factors have a major impact for the second screen use case, essentially meaning that when engaging with one screen $100 \%$ of the 'other' screen is likely to be in the far peripheral of the user's vision.

As opposed to visual information, auditory information is omnidirectional, however, our perception is still limited by our cognitive capabilities. Dichotic listening experiments, motivated by the famous cocktail party effect (see [7] for a comprehensive review), are a clear indicator that we are unable to effectively perceive

1 Those which do not effectively notice potential predators in their peripheral generally do not pass on their genes. 


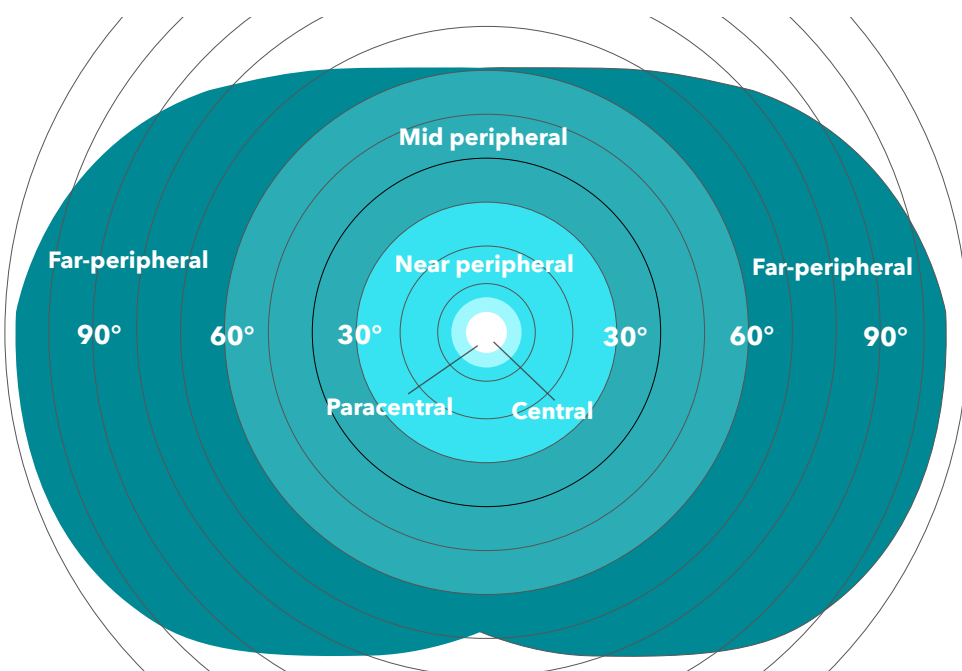

Figure 5: An approximation of a human binocular vision, adapted from Wikipedia Commons $^{2}$

multiple auditory information streams simultaneously. This, has a high impact on our (previously mentioned) poor simultaneous comprehension [123] of textual and auditory information combine to create two separate digital perceptual worlds.

Clearly, the consumption of multiple visual and auditory streams is likely to be tasking, and therefore much research has focused on understanding and remedying this. Media multitasking (the simultaneous use of multiple media streams) [135], has become the norm for many people, especially for television. Much research has investigated the effect that engaging with multiple information streams has on our retention of information. Patterson, for example, studied media multitaskers when revising for exams [110], finding that those who studied for exams while media multitasking were far more likely to score poorly in exams. Further, Brumby et al. [24] looked at the effect of working with the television on. They 
found that when working with the television, we reduce our involvement with the television programme (and therefore lose the chance to relax).

In terms of retention of information, as discussed by Wallis [142], in contrast to television, reading is self-paced and for at moments where we can take a break to stop and reflect deeply on an event. Television broadcast media is, however, is not self-paced. Due to its continuous nature, one must constantly attend and is given little reprieve for reflection. Concurrent streams of information add extra mental effort to engage and generally results in poorer performance in comprehension tasks [3]. Moreover, the way we engage visually is changed. For instance, in 2011 Brasel and Gips [20] looked at how we divide our attention between a computer and a television concurrently and found that people switch between media at an extreme rate, averaging more than four switches per minute, with little knowledge of their own switching behaviour.

The $\mathrm{BBC}$, from their experience of trailing applications, note that there is a continuum we must be aware of when considering second screen applications in terms of attention. While companion applications are designed to entertain, broadcasters are aware that there is a limit to which they should distract from the initial programme for the dual-screen experience. In addition to the visual and auditory demands of the user, interactivity is also a vital factor to consider the users' requirements to engage with the application. As shown in Figure 6, it is clear that certain interactions, such as short text and images, are likely to require little concentration to engage with, but are likely also not stimulating in terms of interactivity. Interactions such as play-along games are likely to require great concentration to take in, both in terms of interaction cost and concentration. There is a tradeoff here second screen content which falls into the upper right quarter of Figure 6 is likely 
to detract from the programme, whereas materials in the bottom left may not be enough stimulation.

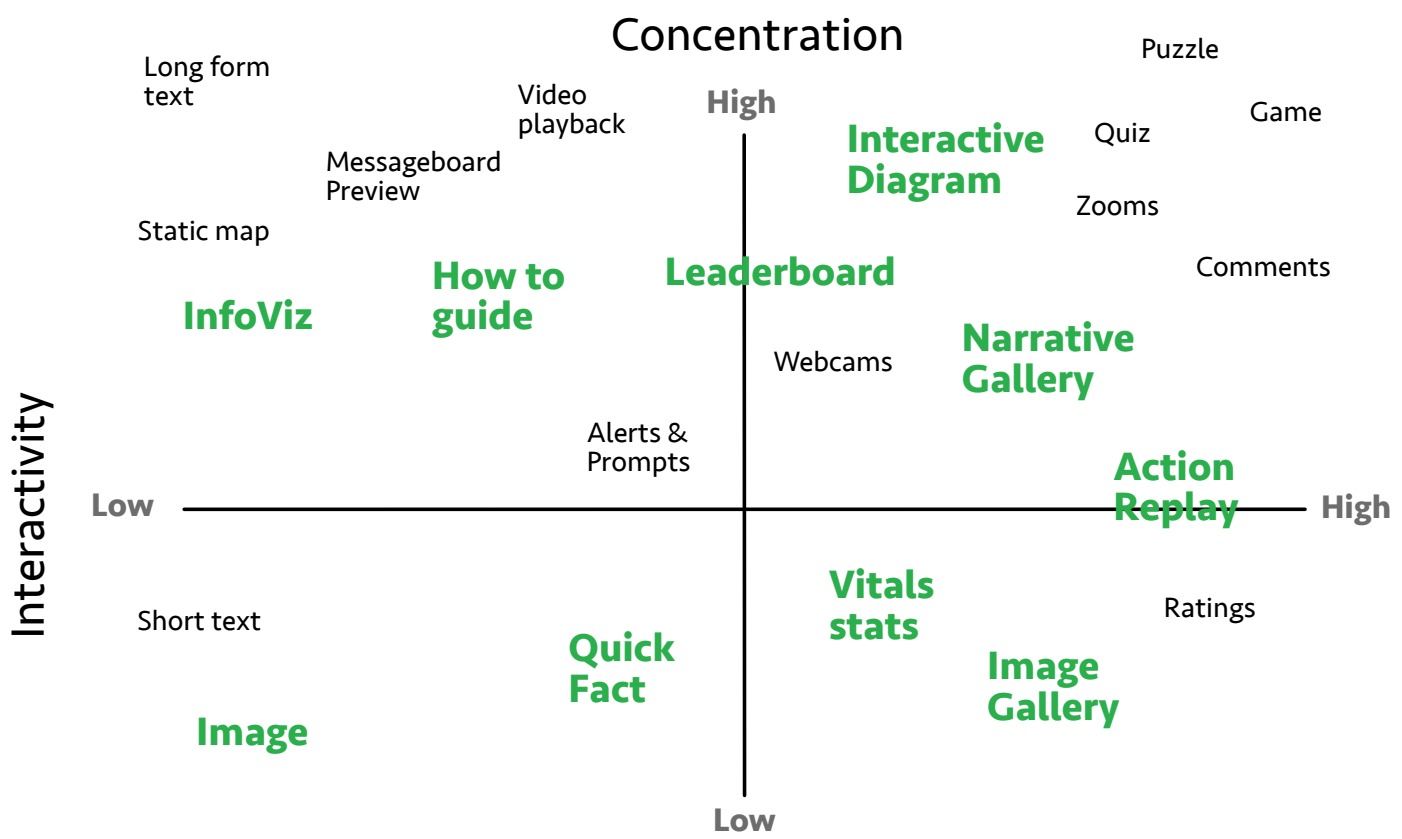

Figure 6: Map of interactivity and concentration in the second-screen use case, adapted from [72], to provide examples of existing work. Green indicates common trends in second screen apps.

\subsubsection{Studies of Cross-Device Attention}

In addition to the rich ethnographic and deployment based research, there have been a variety of empirical lab studies around second screening towards understanding the fundamental attention constraints of the scenario. These have predominantly taken the form of lab studies. Before second screens were ubiquitous in the living room, Robertson et al. [119] in 1996 explored the effect of a second screen in conjunction with a television. This early research showed that the distribution of information across the devices was powerful. The superior visual properties of 
the television should be properly utilised in tandem with the handheld device, such that the focus can remain on the handheld device's stronger interaction capabilities; that the device's shortcomings are made up for by the other screen. In addition, they note that device continuity is critical. One must not lag the other. The findings from this paper have been built upon and cited extensively by the recent boom in the dual screen use case powered by companion applications.

In the recent growth of the dual screen scenario, these findings have been extended by more empirical studies. For example in 2012 Holmes et al. [66] looked at visual attention across screens via eye tracking. They found that when users interact with a synchronised second screen device they view the second screen content somewhere in the region of $30 \%$ of the time. Moreover, they found that this viewing was sustained even when the content was not being refreshed, and that for each push of new content there were spikes in attention. Work by Brown et al. [23] further investigated this phenomenon. They considered methods for eye-tracking across the screens and note the significant difficulties in doing so. In general, they provide insight into the attention switching and percentage of gaze such dual-screen scenarios attract. They detail a variety of complex and nuanced behaviours that such scenarios imply with regards to attention and interaction.

Further to the increased visual disparity between the two screens, Brown et al. [22] also reported that the users' attention often switches between the two devices due to a number of factors. The most obvious of these are the content appearing on the tablet computer dragging attention toward it, or the user simply finishing attending to the companion content. However, it is evident that even when the content is not updating, both devices command command attention. For example: 
- Scene switches - for example an indoor scene changing to outdoor may catch the users' attention and take it from the tablet to the TV. This phenomena is discussed in more detail by Valuch et al. [136].

- Inactivity - in both displays, inactivity, such as indoor scenes with talking heads and not much happening, tends to cause shifts in attention.

- Contextual cues - Dialogue in the show (unintentionally) bringing their attention towards the television, for example the presenter saying "Woah! Look at that!".

In addition to Brown et al. and Holmes et al.'s work on multi-screen eye tracking, Vatavu and Mancaş propose a visual attention toolkit [140]. In this toolkit, they introduce a set of nine measures to characterise viewers' visual attention patterns for multi-screen TV. They propose quantitive and qualitative metrics by which to consider visual attention across screens. The work evidencing multi-screen interaction is a clear indicator that the dual-screen use case is thwarted with issues relating to attention - complex auditory and video streams competing for attention are clearly going to have a major impact on users. Further, Chorianopoulos et al. [27] note the importance of proper UI distribution for multi-screen contexts in their initial investigations.

To better understand the effect of such multi-screen scenarios, lab studies such as that by Kallenbach et al. [74] - before such devices proliferated the living room are clear evidence of the great impact extra information to a programme creates with regards to message processing ability, and cognitive workload evidenced by psychophysiological measurements and cognitive workload measure. 


\subsubsection{Display Discontinuities}

Such discontinuities in multi-screen systems are relatively well documented, and are likely to be a major impacting factor on dual screen UX. However, this work mostly focuses on computer monitor setups [57] and task-driven scenarios [114]. Lab studies on dual-screen TV, such as the work of Brown et al. [23] and Holmes et al. [66], imply that by creating a large physical discontinuity (refer back to Figure 4) between the TV and the handheld device we create a split-attention scenario. This means that users must manage their own attention, which adds additional attention requirement.

Further to the display discontinuities associated with this use case, we also add the factor of multimodality - typically audio and video on the TV, combined with textual and graphical information on the tablet. The multimodal aspect of the scenario is likely to have a major impact. As we are generally poor at simultaneous comprehension of text and auditory information [123], the concurrent textual engagement from social media and information search on the device, and the auditory stream from the video media are likely to result in increased mental effort. As found by Lin et al. [86], TV, even when on in the background, affects reading comprehension significantly. Moreover, it is strongly influenced by genre, for example, more factheavy news programmes was found to be more detrimental. In addition, Basapur et al. [11] note that content unrelated to the TV material itself may be generally distracting to the viewer. 


\subsubsection{Information Overload}

The notion of 'information overload' is a recurring theme in the literature on companion applications. For example, Geerts et al. [53] who noted that, when trialling a dual-screen application for a programme, users were often overcome with the stimuli, and found that they had to return later when the pace of the TV programme had slowed down. A problem when returning later, however, is the fact that the information may no longer be relevant to the TV programme, and may only act as a recap. Further, Basapur et al. [10] noted in their deployment of a companion app that, although, generally, the participants acted favourably towards supplementary content, they noted that such scenarios did not embellish viewing when they wished to 'unwind'. This was because the second display warranted too much visual attention. To progress this design space and alleviate issues associated with attention over screens, work has looked at the distribution in attention between a TV and a companion device $[23,66]$ by means of eye tracking. Both studies found that, in a typical use case, visual attention tended to be largely focused on the more motion rich TV.

Though there is some evidence that viewing TV alongside supporting content can be taxing on attention, there is little empirical evidence of the impact this phenomenon actually creates for our users. As all studies of the attention required to interact with such scenarios have been conduced in the lab - with a focus on eye tracking - we are unable to gather a holistic picture of how this affects the use case for everyday viewing. Therefore, in the remainder of this chapter we contribute to the field by conducting two empirical studies towards gathering an impression of the issues users face, and the strategies they use to overcome the associated mental 
effort. We do so with a vision of informing the design of multi-screen experiences that consider our attention capabilities, towards enhanced dual-screen UX.

No work currently looks at the issues around second screen attention when browsing second screen content from the perspective of the users and their regular viewing habits. If we are to consider the design of applications which support the complementary browsing of second screen materials, it is essential that we understand this phenomenon better from the viewers' detailed perspective. Therefore, we now describe two studies conducted towards investigating this from the users perspective - through an online survey and in-depth interviews with second screeners, we looked to uncover reflective insight from a large population.

\subsection{THE VIEWERS' PERSPECTIVE: AN ONLINE SURVEY}

By using a survey we aimed to gather a broad snapshot of the second screen landscape - to capture users' impressions of their own attention to determine how they understood their own usage of second screens, with particular regard to how they feel they impacted their attention. As noted by Lazar et al. [84] surveys are useful for capturing a large, broad set of data in an unobtrusive manner.

\subsubsection{Experiment Description and Participants}

As a starting point, we surveyed people who regularly watched TV and used a second screen at the same time to engage with the complementary material. We recruited our participant pool from mailing lists at Swansea University, seeking people who actively browse complementary content or social media while watching television. We sent out a request to both staff and students, receiving 364 re- 
sponses in total. Though we explicitly noted in the email we were interested in those who browsed complementary materials, we filtered out those who did not with the qualifying question "Do you ever engage with additional content related to the show you are watching on a secondary device, such as a tablet computer or smartphone?", reducing the total number of participants for our analysis to 260 . A caveat around our results presented here is that we used a predominantly student-based demographic of second screeners, meaning that our sample is relatively young and are predominantly digital natives.

Of our participants, the average age was $21.4(\max =42, \min =18)$, of which 151 identified as male, 108 female and 1 gender fluid. A large number of people also agreed with the fact that they are easily distracted by their mobile devices (median =4) -85 strongly agreed, 61 agreed. However, the participants tended to side more towards neutrality when responding to statements about getting easily get absorbed and losing all sense of time when watching TV (median $=3$ ). In addition, most participants strongly agreed (76) or agreed (77) when presented with the statement about often having the TV on in the background while doing other things.

As the participants were from a UK university they were from a number of countries and spoke a variety of languages, but were predominantly English-speaking British nationals. In addition, our participants were frequent touch screen users $79 \%$ (205) participants 'strongly agreed' that they used touch screens often. We also asked the types of second screen viewing they undertook - the key coping strategies of our participants were that they searched for content related to the production of the programme (104) - actors, directors, plot lines, etc, and for facts 
related to the programme (53). The participants showed a strong preference for mobile devices such as smartphones (138), but also used tablets (28) and laptops (36).

We were interested in finding out how second screening on a mobile or tablet while watching had an impact on their experience of one or both of their devices. Through the online survey, we asked participants specific questions to whittle down the issues when they second screened. We asked questions associated with their mental effort; questions that probed how certain types of programmes or applications affected their dual-screening; and to what extent different stimuli types (auditory, visual) interact and affect the users. The questions and respective results are depicted in Table 1 . For questions 8 and 11 we left free text fields and asked participants to provide explicit examples when necessary. Regarding the questions asked to the participants, we asked the following questions (referred to Q14 through Q26) 3 to the participants to understand the following factors of the users' current experience with second screening (full questionnaire in Appendix A).

- Q14 $\left(\mathrm{S}_{1}\right)$ - How much mental effort they perceive when engaging with television;

- Q15 (S2) - How much additional effort is required to watch television and engage with a mobile device in general;

- Q16 $\left(\mathrm{S}_{3}\right) \&$ Q17 $\left(\mathrm{S}_{4}\right)$ - whether specific behaviours such searching for related information or social networking have a particular impact on the user;

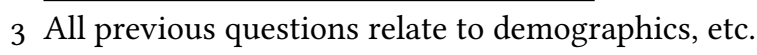


- Q18 (S5) \& Q19 (S6) - to which extent users are aware of the audio-visual information stream from the television while second screening, and which one is more prominent;

- Q20 $\left(\mathrm{S}_{7}\right)$ - whether users are aware of visual content on their mobile device updating while watching television;

- Q21 \& Q22 (S8) - to what extent users feel programme types effect the the mental effort it takes to engage with second screen material, noting particular genres;

- Q23 (S9) \& Q24 (S10) - whether users believe they are more likely to engage with the television or the mobile device when second screening;

- Q25 ( $\left.\mathrm{S}_{11}\right)$ \& Q26 - To understand if users were more likely to second screen for some programmes rather than others, and if so, which ones.

The statement number is also noted here to so that the reader may refer to the questions in Table 1. 


\begin{tabular}{|c|c|c|c|c|}
\hline Statement & Measure & 1 & 2 & 3 \\
\hline S1) When I watch TV I experience & Mental Effort & 19.2 & $34 \cdot 2$ & 34.6 \\
\hline S2) When I watch TV with a secondary device I experience & Mental Effort & 10.0 & $35 \cdot 4$ & 33.8 \\
\hline $\left.\boldsymbol{S}_{3}\right)$ When I watch TV and search for related information this requires & Mental Effort & 8.1 & $37 \cdot 3$ & 29.6 \\
\hline 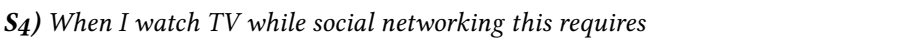 & Mental Effort & 13.8 & $35 \cdot 4$ & 28.8 \\
\hline$\left.S_{5}\right)$ While engaged with a second screen I am aware of visual content on the TV & Agreement & 3.5 & 19.6 & 26.5 \\
\hline S6) While engaged with a second screen I am aware of auditory content on the TV & Agreement & 4.2 & 12.3 & 23.8 \\
\hline $\left.\boldsymbol{S}_{7}\right)$ When I watch TVI am aware of visual information on my handheld device & Agreement & 3.1 & $15 \cdot 4$ & 20.0 \\
\hline S8) Certain types of programme require more mental effort when second screening & Agreement & 0.0 & 4.6 & 6.5 \\
\hline S9) I am likely to focus on the second screen content more than the TV & Agreement & 5.8 & 22.7 & 32.7 \\
\hline S1o) I am likely to focus more on the TV programme when I second screen & Agreement & 5.0 & 28.5 & 36.9 \\
\hline $\left.\boldsymbol{S}_{11}\right)$ I am more likely to use a second screen for some programmes than others. & Agreement & $7 \cdot 3$ & 9.6 & 13.1 \\
\hline
\end{tabular}

Table 1: Data from the online questionnaire: the numbers reported refer to the numbe $=260$ ) who responded with the stated answer, along with the median of all responses. late to the users' perceived level of low/high mental effort (low mental effort $=1$, high and statements (S) 5 to 11 refer to their agreement with the statement $(1=$ strongly dis agree). Darker colours indicate a higher frequency/number. The final column, $\widetilde{x}$, denote The questions have been slightly simplified for this table, however, a full list of quest Appendix A. 


\subsubsection{Quantitative Results}

We first analyse the quantitative response from the survey, and investigate differences in the participant's evaluation of their viewing using statistical techniques. We use Wilcoxon signed rank for comparisons, and report Spearman's $\rho$ for correlations. Then, we frame this data in the qualitative feedback we received online using thematic analysis.

The results for the quantitive online survey are presented in Table 1. As a baseline, participants noted that TV required very little mental effort to engage with $\left(\mathrm{S}_{1}\right)$. However, with the addition of a handheld device (S2) the reported mental load increased significantly $(Z=3.24, p<0.001)$. In addition we found a positive correlation $(\rho=0.30, p<0.001)$ between general mental load watching TV ( $\mathrm{S}_{1}$ and $\mathrm{S}_{2}$ ). This difference was slightly smaller for social networking $\left(S_{4}\right)(Z=2.64, p=0.004)$, and enlarged when searching for related information $\left(S_{3}\right)(Z=4.17, p<0.001)$. Indeed, participants reported that they feel significantly more mental effort when searching for related information than when social networking $(Z=2.00, p=0.024)$. Finally, in general, those who did find social networking mentally intensive also found browsing mentally effortful, shown by a positive correlation between $\mathrm{S}_{3}$ and $\mathrm{S}_{4}(\rho=0.49, \mathrm{p}<0.001)$.

When reflecting on their dual-device usage, participants stated that when viewing their secondary device they are significantly more aware of the TV's auditory channel (S6) than its visuals in their peripheral $\left(S_{7}\right)(Z=3.88, p<0.001)$. In addition, despite the fact that we found no significant preference for which device the participants were more likely to focus on while dual-screening (comparing S8 and S9), participants noted that they were significantly more likely to notice changes 
in information updating on the secondary device (comparing $\mathrm{S}_{5}$ and $\mathrm{S}_{7}$ ) than on the $\operatorname{TV}(\mathrm{Z}=2.22, \mathrm{p}=0.013)$.

Participants had strong impressions about genre, or types of programme when it came to second screening (S8), and a large portion (median $=5$ ) either agreed with, or strongly agreed with the statement "Certain types of programme require more mental effort when second screening". Moreover, they generally noted that they were more likely to use a second screen for some programmes, but not others. There was a correlation between those who thought that certain programmes required more mental effort and those who were more likely to second screen for some programmes $(\rho=0.30, p<0.001)$. Finally, to determine the inter-participant differences we investigated correlations in demographics information and the reported viewing usage. With regards to hand held device usage, we found that those who often use touch screens $(\rho=0.24, \mathrm{p}<0.001)$ and those who feel they are easily distracted by touch screen devices $(\rho=0.24, p<0.001)$ are more aware of what is happening on the second screen as they view ( $\mathrm{S} 9)$.

\subsubsection{Qualitative Results}

As indicated by the quantitive survey data, participants felt that the genre, or the type of programme strongly impacted on the mental effort required to dualscreen. When asked what types of programme requires more mental effort to dualscreen the participants noted specific genres and themes. The most mentioned were drama (20.7\% of participants), for example P165 - "Shows with a more complex story line and engaging characters such as, documentaries and drama series", and documentaries (20.3\% of participants) - "Documentaries [as] the topic can change while your [sic] looking at the topic in more depth" $\left(\mathrm{P}_{7}\right)$. Genres such as comedy (5 parti- 
cipants (1.9\%) and sport (5 participants) were mentioned less and generally referred to as 'background viewing'. With regards to themes the largest noted impactor on mental effort was a complex plot (8.4\% of participants) - "Things with complex plot lines, such as Game of Thrones” ( $\left.\mathrm{P}_{13}\right)$. Subtitles were also noted as a major factor by 13 (5\% of participants), and wholly attributed to those who watch foreign films or anime - "If there's subtitles! [I] can't really use a secondary device at all. (P140).

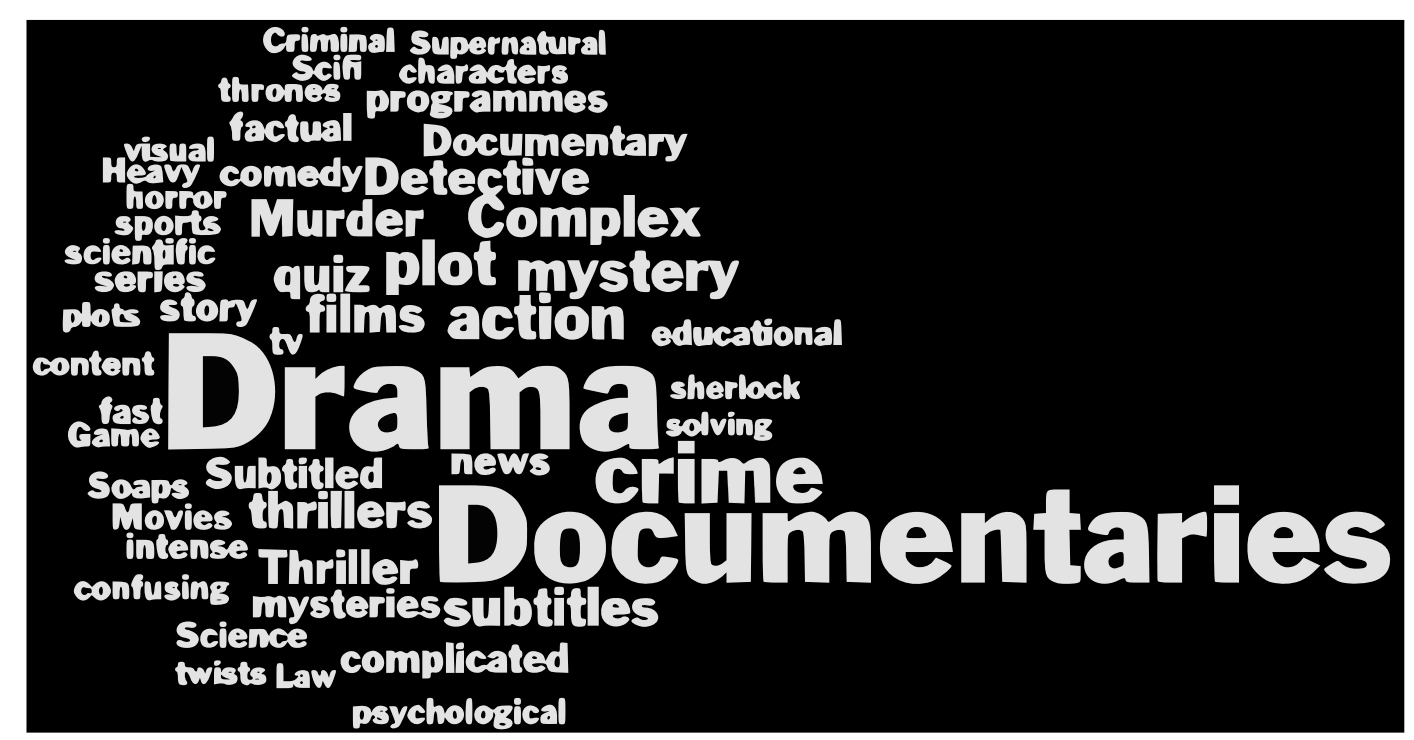

Figure 7: This wordcloud represents the 50 most frequently noted phrases that users stated when asked what types of programme require more mental effort for them to second screen alongside. The size of the words is scaled to their frequency - the most frequent being documentaries and drama and therefore the largest.

When asked to freely expand on what types of programme participants were more likely to second screen for we saw a split - those who noted they actively second screen for specific types of programme, and those who simply use a second screen to fill the lull in attention during boring programmes they dislike, or uneventful moments in programmes. Those who actively second screened were likely to do so for searching extra information about actors or actresses (4.2\% of participants), 
and searching for supporting information (6.9\% participants). Sports, drama and documentaries were prominently noted as programmes participants would undertake additional information search for. In contrast, many participants noted they second screened to fill some void in attention. In general, this was done alongside programmes with simple plots (10.7\% participants), or programmes that the participants had seen before (9.6\% participants), or quite simply programmes that the participants perceived as boring, or they disliked (8\% participants) - "[I'm] more likely to second screen for boring, slow-paced or reality programmes, or programmes I just have on for the sake of it. As it doesn't matter if you miss a bit" ( $\left.\mathrm{P}_{141}\right)$.

\section{$4 \cdot 3$}

DISCUSSION

From the quantitive data it was evident that to engage with TV on its own requires little perceived mental effort. However, with the introduction of a hand held device we found that the required effort increased significantly. Moreover, the significant correlation between the reported mental effort when watching TV in general and when dual screening implies that those whose attention is burdened by TV content, are likely to also experience these issues when dual-screening. The additional mental effort reported by the participants to engage with related content as opposed to social networking may be explained by the fact that tangential search of information is an active search process. Such findings support the need for designated companion applications that support users when watching TV programmes (c.f. $[11,105])$ - essentially reducing the need for users to formulate and pursue knowledge query.

Participants generally reported noticing the auditory channel of the TV more this is not surprising as audio is omnidirectional, and one must actively engage 
with the visual stimuli to view it. However, what this implies is that when engaging with content participants may be monitoring auditory streams. As textual comprehension drops significantly when monitoring auditory information [123] this is likely a major impacting factor on the perceived additional attention cost of the dual-screen scenario.

As in the work of Basapur et al.'s [10], genre, and the type of programme, had a clear impact on the participants' perceptions of the dual-screen scenario. With regards to the mental effort required, we saw a moderately strong correlation between the perceived mental effort required of certain programmes and the agreement that they were more likely to second screen for some programmes suggests that their usage of second screens in certain programmes is strongly impacted by perceived mental effort. Therefore, it is vital to reflect on our qualitative data to get a full picture of perceived mental effort for given programme types.

The qualitative data strongly suggests that certain types of programme impact strongly on the dual-screen experience. In terms of genre the participants tended to cite material that required constant attendance because it was fast changing or cognitively involving (documentaries), or if the experience was largely dependent on prior knowledge of prior or current developments (drama). Our findings, here, begin to shed some light on RQ1. We see from this survey, as expected, that the addition of a device to a television programme adds additional attention. However, we also see clear implications for genre and the effect of engaging with related content. For example, when users are engaged with some active search process, the competing streams result in an additional cognitive burden. 
Now that we have an impression of the types of programmes and scenarios that cause mental overload, we probe deeper to gain qualitative insight. We report on semistructured interviews we conducted towards understanding further what impacts our attention, along with how we manage our attention to avoid and overcome the attention bottlenecks associated with this use case.

\subsubsection{Method}

The main aim of the interview was to get an impression of the participant's second screen habits, and to investigate their experience when managing their attention while second screening. It was conducted in the form of a semistructured interview, where the test coordinator was free to probe deeper into certain points, and to be tangential in questioning. However, the same three main questions were asked:

1. Do you interact with devices while watching TV? If so, what sort of things are you looking at? And, how do you adapt your TV viewing?

2. Do you get information overload when viewing two devices? What sorts of content trigger this?

3. When would you sacrifice not looking at your secondary device to look at the TV, and, when would you sacrifice not looking at the TV to look at your mobile device?

We asked Question 1 to, firstly, gather a picture of the appropriateness of the subjects. As we chose exclusively second screeners for this study, we wanted to make 
sure this was the case. Then, we wished to gather a question of the secondary content the users were viewing and how it affected their viewing, to establish any themes about the types of content one would adapt their television viewing for, and how.

Question 2 was asked to determine if users get some degree of information overload when viewing content and, if so, what types of content cause this. This was to gather an idea of how many users find second screening problematic and to uncover the particular types of content (TV and mobile device) which cause this. Here, the interviewer probed points the users made further, in keeping with the semi structured nature of the interview.

Finally, in Question 3, we wanted to understand the methods use to cope with attention overload - provoking them to explore what types of content they are likely to miss over others. Again, digging deeper to points with further questioning. The interviews were then transcribed and, thematically analysed, to uncover common themes in the data relating to methods for adapting TV viewing, content that triggers information overload, and when they would sacrifice not viewing a device.

In terms of participants for this experiment we recruited self-reported second screeners from an agency which the BBC regularly use to recruit participants (8 participants), and from within the BBC itself (from departments unrelated to this research, such as administration) (12 participants). There were 20 participants, aged between 22 and 65, with an average age of 39.4 (SD = 13.04). 11 participants identified as female, and 9 male. On average the participants reported viewing 2 hours and 5 minutes of TV per day. 


\subsubsection{Results}

Common strategies involved searching for information related to the programme (such as actors/actresses and places), social networking (often following Twitter trends related to the programme), or even searching for the next thing to watch. It was clear that some participants viewed the second screen considerably more than others, with some having the focus of the experience as the TV - "If there is something on the telly that is really gripping then I would tend not to fidget with my device" $\left(\mathrm{P}_{5}\right)$, and others having the focus of the experience on the tablet - "[I'm] constantly on the iPad while watching telly" (P10).

Of the participants who engaged with devices while watching TV, 14 (74\%) noted that they often get some degree of information overload when trying to split their attention between the two screens. Of these participants, they normally went on to discuss one of two things - either how they would mitigate against missing bits of the TV programme (or content on device), or how they would compensate for losing information.

Common methods from the users for mitigating overload were: actively putting aside the device in more engaging parts of the programme (6 participants), pausing to read or view something on a second device (6 participants), or trying to focus on one screen and dip in and out of the other dependent on what attracts their attention (9 participants). Of those who tried to dip in and out of content we noted two main viewing habits: those who view the TV as a primary device ( 5 participants) generally wanted an 'experience', and to fully take in the TV programme as it was created, for example $\mathrm{P}_{3}$ - "If I am watching TV, I am watching TV. I tend 
not to get distracted and tend to fixate on one thing. I guess I go into some kind of bubble 'experience' about what is on the TV. I want a full on experience".

When compensating for information lost (i.e. the user has missed some information), some common methods emerged. The most frequent comments were related to rewinding the programme to catch up on key events (8 participants) they missed while interacting with their devices. This appeared to be a very common occurrence, with more than $50 \%$ of the participants who dual screened undertaking this. For example Participant 4 noted being distracted by their phone - "Sometimes I feel that I am distracted by my mobile phone and then I find that I have to rewind". P6 went further -"I mostly watch dramas and films - things I was not expecting, like "Oh, I'd better go back and find out what they actually said!".

Rewinding was more common in programmes where the plot was integral, and where subtle details could not be overlooked - "If it's a show that I really like that goes really fast, like 24, then you can't really not watch that, or you can rewind it" (P6). Rewinding was not only a strong trend, but one that clearly totally transforms the modern day viewing experience - "Yeah, well, you can rewind it [TV] now, but it can take twice as long to watch a programme. Because obviously, you're not paying attention and you have to go back and check what you were watching. I don't know what I would have done 10 years ago..." (P6). Other key methods involved people looking up information after the fact, re-watching the entire programme at a later date ( 2 participants), asking who they were with at the time (1 participant), and turning down the TV volume (2 participants).

Conversely, the participants who focused mostly on the tablet used it as one would with a newspaper - reading intensely, but occasionally flicking attention towards 
the TV, normally by monitoring developments in the auditory channel - "The Voice doesn't need any concentration whatsoever, so if that is on I just keep an ear to what is going on" $(\mathrm{P} 18)$ - " if it's something that I'm watching and I am really engaged in it - where the visual is important and you can't pick it up from just the audio channel" ( $\left.\mathrm{P}_{5}\right)$; "I think I probably sacrifice looking at the tablet and look back at the TV if it was obvious that something exciting was going on with noises" (Р20).

Finally, concerning genre effects - often our questioning closed in on more details, and it was clear that certain types of programme afforded different styles of viewing: TV as focus; or secondary device as focus. Programmes that users tended to want their full, undivided attention on, were films, documentaries and dramas. A very common trend was participants noting that they would not want to interact with secondary devices when there was a plot. This was something noted by several participants, for example, $\mathrm{P}_{1}$ - "If it's something that has a narrative, like a film, I am less likely to be doing stuff [on my secondary device] I suppose" and $\mathrm{P}_{5}$ - "I've been watching Wolf Hall lately. So for something like that I would be totally engaged and not do any second screening". On the other hand, programmes participants would have on in the background included sport and cooking programmes. There was some evidence that participants also second screened dramas and documentaries. However, due intense plots and mental load associated with such viewing many of the participants reported rewinding and pausing - "[Britain's biggest primary school]... It's like [the programme] Educating Yorkshire, but with primary school children. And I was looking at what people were saying on Twitter and I kept on having to rewind it" (P16). 


\subsubsection{Discussion}

Though it is clear that the type of viewer brings a lot to the dual screen experience, it is likely that genre of programme also has a major impact. The data suggested that certain types of programme afforded certain dual-screen viewing styles. The participants dealt with the information overload either by mitigating (most commonly pausing), or compensating (most commonly rewinding) for the information they lost.

Some participants were more able than others to use the two displays effectively; for example by monitoring the auditory channel in the background while watching TV. To compensate for information lost, most commonly participants rewound programmes. The prevalence of rewinding indicates that many users are missing integral points in programmes. It could be suggested that as TV is now often ondemand, rewinding has become a convenient affordance for those who engage with second screens extensively while watching TV. Further, this may suggest that if we were not able to rewind users may engage with the content more by devoting more attention to the main screen material. To mitigate, pausing was the most common user action.

In terms of answering RQ1 - the main bottlenecks facing users - it is clear that some genres were of clear issue for our participants when they second screened, and this clearly impacted some more than others in terms of mental effort. We therefore suggest that, when designing multi-device experiences, designers strongly consider the perceived complexity of the TV programme. It is evident that pro- 
grammes with complex plots, such as dramas and crime thrillers, should be treated with care and consideration as the users' focus is most likely to tend more to the TV as they wish not to miss vital information. Moreover, programmes with cognitively involving materials, like science documentaries, will likely have a similar effect. We should design our systems with this in mind to compensate for such factors, and strongly consider trialling materials before public distribution to probe perceived cognitive load when designing multi-screen experiences.

Though certain genres clearly do have a major impact, it does not necessarily mean that they cannot be enhanced by companion content. We recommend that when designing for potentially complex programmes, designers carefully choose the points at which they introduce/refresh second screen materials (especially time-relevant materials); strongly considering its presented complexity. An additional approach may be to tackle the issue head on, and provide companion applications that directly allow users to mitigate the complexities in plots of dramas (as in [105]), or the intricacies of information-rich documentaries (c.f. [38]) with simplified summaries of the complex information in the programme.

When we reflect on the coping strategies from the users in the interviews, we see some clear design implications - if we are to provide additional content that may tax attention, we should take note of these coping strategies. For example, allow pausing/rewinding the experience from the app to fully engage with the companion materials. Or, even to support second screen viewing during lulls in attention in the main programme to promote the users' ease in switching back and forth. 
Clearly, when we consider the design of such systems we need to consider the spectrum of users and use cases. It is clear that certain experiences will have different goals in mind. Whether this is promoting information search, free browsing of the web, or social networking we should strongly consider the different types of viewing and viewers. Over our two studies we were able to categorise our participants into each of the following personas, or 'viewer types' to allow those designing multi-screen experiences to do so with a stronger sense of audience:

- Full engagement with TV - those who wished to view the TV and do nothing else; to be immersed in the TV content and to not engage with a mobile device. Though we evidently did not analyse these participant's data, it is still clear that many users do not second screen - as suggested by our number of participants dropping from 364 to $260^{4}$.

- Symbiotic dual-screen experience - those who used the tablet to directly complement the TV experience, using dedicated companion apps or websites, viewing related twitter hashtags, or exploring related online resources.

- Newspaper viewing - those who viewed the tablet as one reads a newspaper - i.e. focusing on reading things different to the TV content, but allowing their attention to be sidelined by the auditory channel of the TV and shifting their gaze to the TV at key moments.

- Fish tank viewing - those participants who noted only using the TV as a background to their devices. Similar to how one may have a fish tank in the corner of a room for company and decoration.

4 As previously noted, these participants were dropped from the analysis as did not meet this initial requirement. 


\subsection{CONCLUSION}

In this chapter we have documented substantial evidence in the form of reflections from users on attention habits while dual-screening. By examining this using two methodologies, we have been able to explore a rich set of user behaviours and assess the impact of the use case, and further determine what users to do compensate for the issues this creates.

We believe our insights can further inform designers to better consider human attention requirements, with regards to types of viewers and the way that genres affect users. By conducting an experiment with varying complexity we have been able to confirm and deeper explore the participants' concerns around textual information from the previous chapter.

This chapter complements, and expands on work which looks at genre effects for social TV, such as that by Geerts et al. [50], by offering a richer understanding on the effects of genre for cross device attention. Now that we have a reasonable impression of the types of programmes that may affect users, in our future contributions in this thesis we will ensure that we use a diverse set of genres in our stimulus, to ensure that our findings are as broadly applicable as possible.

We have began to understand the bottlenecks associated with this use case, as outlined in RQ1, however, although this chapter has outlined some general rules for the presentation of content, we have not yet considered how this may be practically implemented beyond the content creators simply tailoring the material.

Though this chapter has delivered some high-level qualitative insight through reflections and interviews, towards answering RQ1 with more quantifiable detail, in 
the next chapter we consider the notion of dual-screen complexity in a controlled lab study so that we may design cross-device materials from a more informed perspective in terms of attention. 



\section{CHAPTER FIVE}

\section{Quantifying Cross-Device Complexity}

So far in this thesis we have considered the experiential effects of cross-device complexity through consideration of the literature, and by gathering the reflections of second screeners through online questionnaires and interviews. This has allowed us to answer RQ1 in terms of genre, second device interaction and has allowed us to outline some brief recommendations for design. However, it has not given us a strong sense of the impact of cross-device complexity in a controlled, empirical sense.

We now turn to from understanding this use case from user reflections, to consider it deeper through empirical investigations in the lab. Although our findings in the previous section hint strongly at underlying concerns about the complexity of certain types of content - i.e., certain genres and types of mobile device content, we have little understanding of cross-device complexity. In this chapter, we turn to consider the impact of varying complexity on a mobile device, and how this interacts with the television content (and its perceived complexity), as a user engages with a second screen application.

As previously explored in Chapter 2, the nature of dual-screening implies that a user's attention will be split, somewhat unpredictably, and much literature outlines the issues around simultaneous consumption of multi-device content. In this 
chapter we offer novel insight into the effect of varying textual and graphical complexity, as quantified by established metrics, on participants' visual attention while watching television.

We first review the literature on quantifying visual in this area to appropriately frame our work, and progress by describing an in-depth empirical study towards establishing the effect of varying cross-device complexity. Our findings, informed by subjective and objective data, can assist in understanding the limits of attention, and the nuanced implications of providing second screen content of a given complexity.

There has been a large amount of previous research studying the visual complexity of computer UI and web pages. Much of this is highly applicable to companion content: Reinecke et al. [116] investigate first impressions as a function of complexity, and Harper et al. [60] investigated visual complexity in webpages and its impact on cognitive load, finding that the main factors for the perception of high visual complexity were high density and wide diversity of elements. Michailiduo et al. [100] investigated a method for calculating the perceived complexity of web pages. Constituent features such as links, images, and tables are measured and compared to a threshold (for example, text $>600$ words would be considered 'visually complex').

We should also consider standardised textual complexity evaluation methods such as the Flesch-Kincaid reading age score [79], which is used by a number of educational, and military corporations to ensure their documentation is of the required 
standards. A useful definition of visual complexity for graphics is by Olivia et al. in [109], attributed to Heaps and Handel [61]:

"Visual complexity is related to how difficult it will be to give a verbal description of the image and how difficult it will be to remember the scene after seeing it for a short time."

Visual information which requires some further knowledge or processing to interpret can be considered more visually complex by this definition, for example how children learn to read digital clocks faster than analogue [49].

\subsubsection{Visual Attention for Dual-Screen TV}

Visual attention, and how it switches between the companion content and the TV, is investigated in the work of Brown et al. [22, 23]. In extension to work by Holmes et al. [66], Brown et al. investigated visual attention by eye tracking participants while they were engaged in a companion content experience. Both studies found that, in a typical use case, visual attention tended to be largely focused on the more motion-rich elements. Further, Valuch et al. [136] investigated the effects of cinematic cuts on visual attention - finding that visual attention is quickly attracted by repeated visual content - and recommend that designers of second screen material should include visual items from the 'primary display' to reduce the time necessary for the shift of attention between the screens.

In [10], Basapur et al. note, in observations from their companion app deployment, that such applications very much support 'active TV' are quite cognitively involving, and perhaps something that someone would not use to 'unwind'. This notion of visual information overload was also observed by Geerts et al. [53] who 
found that viewers were often overwhelmed with the stimuli, and had to return to it later when the pace of the main programme had slowed down (and, therefore, when the companion material had become less relevant). In addition to considering the visual domain Chorianopoulos et al. [27] consider the interaction costs and benefits of providing varying amounts of interactive UI elements across the TV and a tablet.

With regards to complexity across displays on the same physical screen, Kallenbach et al. [74] investigate the effect of extra textual information on TV viewers' visual attention, message processing ability, and cognitive load. In extension, Van Cauwenberge et al. [137] investigated the effect of participants browsing on their secondary devices while watching TV. Providing layers of detail has been shown to be a potentially effective mitigation of these split-attention issues: studies and prototypes have allowed users to dig for more information through web-links [10], interact with graphics for more detail [41], or expand sections of text for more information [53]. Moreover, some developers introduce relevant content at specific points to reduce distraction from the experience $[10,23,53]$. Brown et al. go further [23], and discuss how content may be generated based off a user-driven attention mode, as has been demonstrated in Web interfaces [69].

Though work has investigated visual attention in terms of its distribution across dual-screens, no work currently empirically investigates the subjective or objective impact of the attention split this body of research indicates. Moreover, no work considers a design space for bettering its creation in terms of visual complexity across the foci empirically. Therefore, in this chapter we investigate visual complexity over dual-screens and use our findings to further consider complexity adjustment towards enhanced dual-screen UX. 
A controlled lab-based study was conducted to determine factors which contributed to viewers' overall perception of visual complexity on a main and companion screen and their interaction to form dual-screen complexity. Attention distribution, measured in terms of glances to a handheld device, was also observed as a means to determine potential mechanisms for designing attention between the two elements of the experience. Twenty participants watched four professionally produced and previously broadcast programme clips on a TV screen, accompanied by second screen content, ranging from simple to complex in terms of its complexity. We then evaluated their subjective experience using quantitive and qualitative measures. Guided by the previous literature and similar applications ${ }^{1}$, representative styles of companion content were used, each consisting of supplementary textual information and graphics.

\subsubsection{Participants}

The participants (labelled $\mathrm{P}_{1}$ through $\mathrm{P}_{20}$ ) were aged between 22 and 65, with a mean of $39.4(\mathrm{SD}=13.04)^{2}$. Eleven identified as female, and nine male. They reported viewing an average of 2 hours and 5 minutes of TV per day and $80 \%$ of participants reported that they engaged with portable devices while watching TV to some extent, most commonly to search for information related to the programme or for social networking. Of these participants, half were recruited through an

1 For this we took influence from the BBC's companion application archive, which archives known commercial and prototype companion applications - http://companion.prototype 0 . net/

2 The participants used here are the same as those used in the interviews around the participant's perception of their attention, as described in Section 4.4.2. The reflections of their own usage were captured before running the empirical experiment. 
agency, and half were recruited from the BBC (from departments unrelated to this research). All participants were native English speakers.

\subsubsection{Study Environment}

As we wished to conduct a controlled investigation in a typical setting, we undertook the studies in a user research lab configured as a typical living room. This lab has been used for several years by a major TV broadcaster for similar studies, and ensures as minimum distraction and rich observation of the stimuli effects. The lab's camera equipment was used to view and record the participants and their interactions in situ (Figure 8).
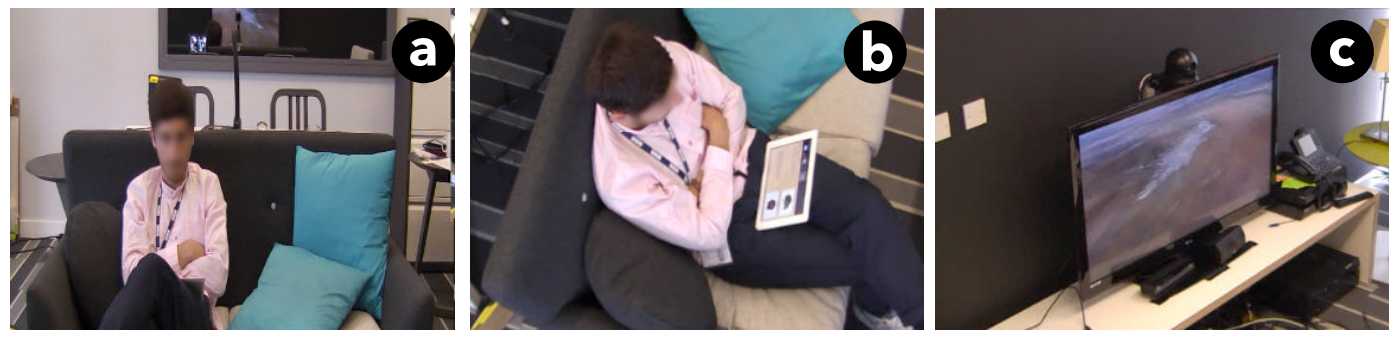

Figure 8: Video observation was captured from three angles: a) directly in front of the participant, to observe reactions such as gaze; b) from above, to observe the device status and reactions from a second perspective; and c) to view the TV screen to maintain a comprehensive record of the content experience.

\subsubsection{Study Procedure}

Each participant completed a demographic questionnaire to allow us to understand our sample population. A sequence of four TV clips (see Figure 2), each accompanied by companion content were then presented, one after another interspersed with post-clip questionnaires. We chose a variety of content to mitigate for genre 
effects [50]. Participants were told that they would be asked recall-based questions about both screens' material. This was to provide instructions to attend to both screens. At the end of the sequence, the participants filled out a questionnaire, and an investigator conducted a semi-structured interview discussing the participant's experience of the different complexity levels. The full questionnaire can be found in Appendix B.

\subsubsection{Stimuli and Tablet Content Design}

The TV content (Table 2) was chosen to be diverse as possible so that we could compare/control the impact of each genre/style of programming. Companion content was displayed at four points, at a diverse set of scenes in the TV clips - some with high levels of dialogue on the TV, others with little; others at points that would require high visual focus on the large screen to understand, in contrast to other visually slower points in the programme, with slower paced editing and more repetitive shots.

\begin{tabular}{lll}
\hline Clip No. & TV Programme & Summary \\
\hline 1 & The Inca: Masters of the Clouds & Inca docu mentary \\
2 & Snooker Coverage: Master's 1/4 Final & Snooker $1 / 4$ final \\
3 & Click (broadcast 5/7/14) & Technology news item \\
4 & Eggheads: Season 15, Episode 18 & Quiz programme \\
\hline
\end{tabular}

Table 2: Table of clips used in the visual complexity experiment

We chose a diverse set of genres due to the evident impact of genre effects, as discussed in the previous chapter. The tablet material was non-interactive graphical companion content, with no audio, which depicted information related to the programme - for example, facts, related images, and tangential news stories. The 
companion screens (Figure 9) were created in four levels of complexity: simple text, simple graphics (' $\mathrm{tg}$ '), complex text, simple graphics (' $\mathrm{Tg}$ '), complex graphics, simple text (' $\mathrm{tG}$ ') and complex text, complex graphics (' $\mathrm{TG}$ ').

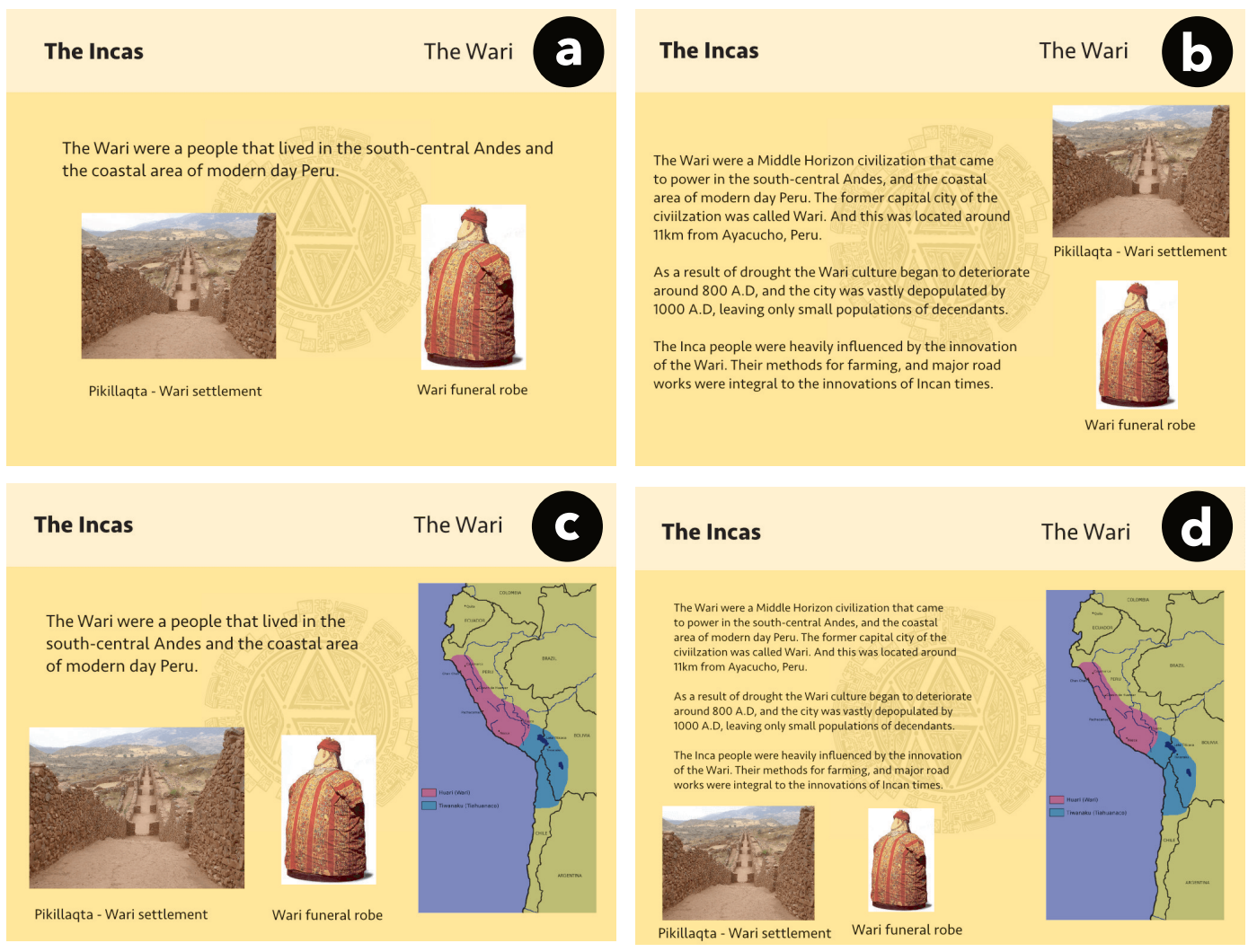

Figure 9: The graphical second screen complexity levels used in the experiment, for the Inca documentary clip (Clip 1). We use lower-case to denote a simple condition, and a capital to denote complex: a) depicts 'tg' - simple text, simple graphics; b) depicts ' $T g$ ' - complex text, simple graphics; c) depicts ' $t G$ ' - simple text, complex graphics; and d) depicts 'TG' - complex text, complex graphics.

In creating different levels of textual and graphical complexities we used standard measures and prior literature to guide our choices. For textual information we used descriptors to consider complexity - the Flesch-Kincaid reading ease score method 
[79], and the length of the textual information. For this we defined two levels of complexity, based on the reading age score of the text and the number of words in the content:

- LOw: a Flesch-Kincaid reading score over 60, which implies the reading age of an average 13 to 15 year old and $<15$ words.

- HIGH: a Flesch-Kincaid reading score below 50, which implies more advanced reading skills and $>25$ words.

We used Heaps and Handel's (aforementioned) definition of graphical complexity. We therefore defined information-rich with complex semantic information, such as graphs and maps, as complex. Non-semantically rich images, such as a picture of an animal, we defined as simple. We used Latin square treatment to balance for the visual complexity levels fairly across all four clips, so that there was an equal number of independent variables (complexity levels) across the TV stimuli. Our content's textual and graphical complexity was designed to be in line with current informational applications used by major broadcasters, for example those in the BBC Companion Archive [28]. In this case, this simply involved us loading the text descriptions as static slide-like content in a similar manner to the BBC's Autumnwatch Experiment [23].

\subsubsection{Measures and Validation Techniques}

For objective data we considered gaze. To determine and record the participant's eye gaze information we used the video analysis software ELAN [130]. Video markup can be used to time-code accurate events and has been used recently, for example, to time-code livingroom activities around the television [117]. We util- 
ised the multiple angle HD video and were able to determine observational data, which was time-coded with frame-by-frame accuracy. When the participant's gaze was on the tablet, the appropriate code was applied and unapplied when the participant's gaze left the tablet (Table 3). To ensure accuracy of the coding technique we utilised a second coder to validate accuracy of timings for a sample of the footage ( $1 / 3$ of the footage). For each sample, there was an average difference of 0.029 seconds $(3.1 \%)$ between coders. Analysis of variance suggests no significant effect between the coders $(F(1,31)=3.5, p>0.05)$, therefore we assume reliable coding using this method.

\begin{tabular}{|c|c|c|c|c|}
\hline Event & Start Time & End Time & Duration & Clip/Content \\
\hline tabletFocus & $04: 28.8$ & $04: 30.3$ & $00: 01.5$ & clip1Content 1 \\
\hline tabletFocus & $04: 32.0$ & $04: 32.7$ & oo:00.7 & clip 1 Content 1 \\
\hline tabletFocus & $04: 35 \cdot 4$ & $04: 36.7$ & 00:01.3 & clip1Content 1 \\
\hline tabletFocus & $04: 38.1$ & $04: 38.6$ & oo:00.5 & clip1Conten 1 \\
\hline tabletFocus & $04: 39.7$ & $04: 40.9$ & $00: 01.2$ & clip1Content 1 \\
\hline tabletFocus & $04: 42.7$ & $04: 43 \cdot 5$ & oo:00. 8 & clip1Content 1 \\
\hline tabletFocus & $04: 43.8$ & $04: 46.0$ & 0o:02.2 & clip1Content 1 \\
\hline tabletFocus & $04: 48.3$ & $04: 49 \cdot 7$ & o0:01.3 & clip1Content 1 \\
\hline tabletFocus & $04: 51.8$ & $04: 53 \cdot 7$ & o0:01.8 & clip1Content 1 \\
\hline tabletFocus & $04: 54 \cdot 3$ & $04: 54.8$ & oo:00.5 & clip1Content 1 \\
\hline tabletFocus & $05: 13.9$ & $05: 18.4$ & oo:04.5 & clip 1 Content 2 \\
\hline tabletFocus & $05: 19 \cdot 3$ & $05: 20.7$ & 00:01.4 & clip 1 Content 2 \\
\hline tabletFocus & $05: 21.7$ & $05: 22.1$ & oo:00.4 & clip1Content2 \\
\hline
\end{tabular}

Table 3: Example of the (rounded) data generated from the video analysis in minutes, seconds and tenths of seconds: - by coding specific events and their duration we were able to determine statistics such as glance length and frequency on the tablet. 
Specifically, we were interested in tablet engagement time - how long a particular piece of tablet content caught their attention away from the TV, calculated by the total duration of glances per piece of second screen content. From this, we could also determine the average length of gaze, to determine how long each point of visual engagement with the content lasts, and how often the participants looked up and down.

After each clip, we administered a Likert scale questionnaire to gather the participants' impressions. Specifically we asked questions related to how hard they felt it was to take in content on the second screen, whether they were missing content on the TV because of the tablet (and vice versa), and how hard it was for them to take in textual or pictorial information. After watching all clips we asked questions related to the experiment as a whole, specifically asking them to reflect on how the detail levels in the companion content affected their overall experience.

We concluded the experiment with a semi-structured interview. For this we asked the same core questions, but the nature of the interview allowed us to probe deeper into specific comments made by the participants. Our main interview questions probed whether they adapted their TV viewing if there was more textual or pictorial information on the second screen; at what points they sacrificed viewing content on the TV, and the tablet; and how much the companion content's relevance impacted the balancing of both screens. We transcribed the data and conducted thematic analysis to probe key trends. 
We now describe the results. With regards to the analysis of the continuous data, we used ANOVA (analysis of variance) to determine if there were general significant effects for conditions, and then conducted post-hoc pairwise comparisons. For ranked data we used Friedman tests to investigate the overall effects of conditions, and then used post-hoc Wilcoxon signed rank tests to evaluate individual differences. In post-hoc analysis we used Bonferroni correction to protect against type I error from multiple comparisons, and set $\alpha=0.05$.

\subsubsection{Visual Data}

Figure 10 depicts a frequency distribution of the length of all recorded glances in the experiment. Most glances tended to fall within the lower range; many less than 10 seconds.

Concerning video analysis tablet engagement time, we took the average of each of the participants' visual engagements for each condition. This is shown in the results in Figure 11. On average, 'TG' (complex text, complex graphics) yielded the longest level of engagement with the second screen: 17.8 seconds $(\mathrm{SD}=7.7)-59.3 \%$ of the time the content was displayed -, and 'tg' (simple text, simple graphics) yielded the least with an average of $10.6(\mathrm{SD}=4.0)$ seconds of time engaged, or $35.3 \%$ of the time the content was displayed. $\mathrm{Tg}$ and $\mathrm{tG}$ yielded an average of 16.8 $(\mathrm{SD}=7.0)$ and $14.7(\mathrm{SD}=6.3)$ seconds respectively.

As the average engagement time for each participant was determined to be of a normal distribution (Kolmogorov-Smirnov indicated $\mathrm{p}>0.05$ ), we conducted analysis of variance on the data. ANOVA indicated that the complexity level had a signific- 


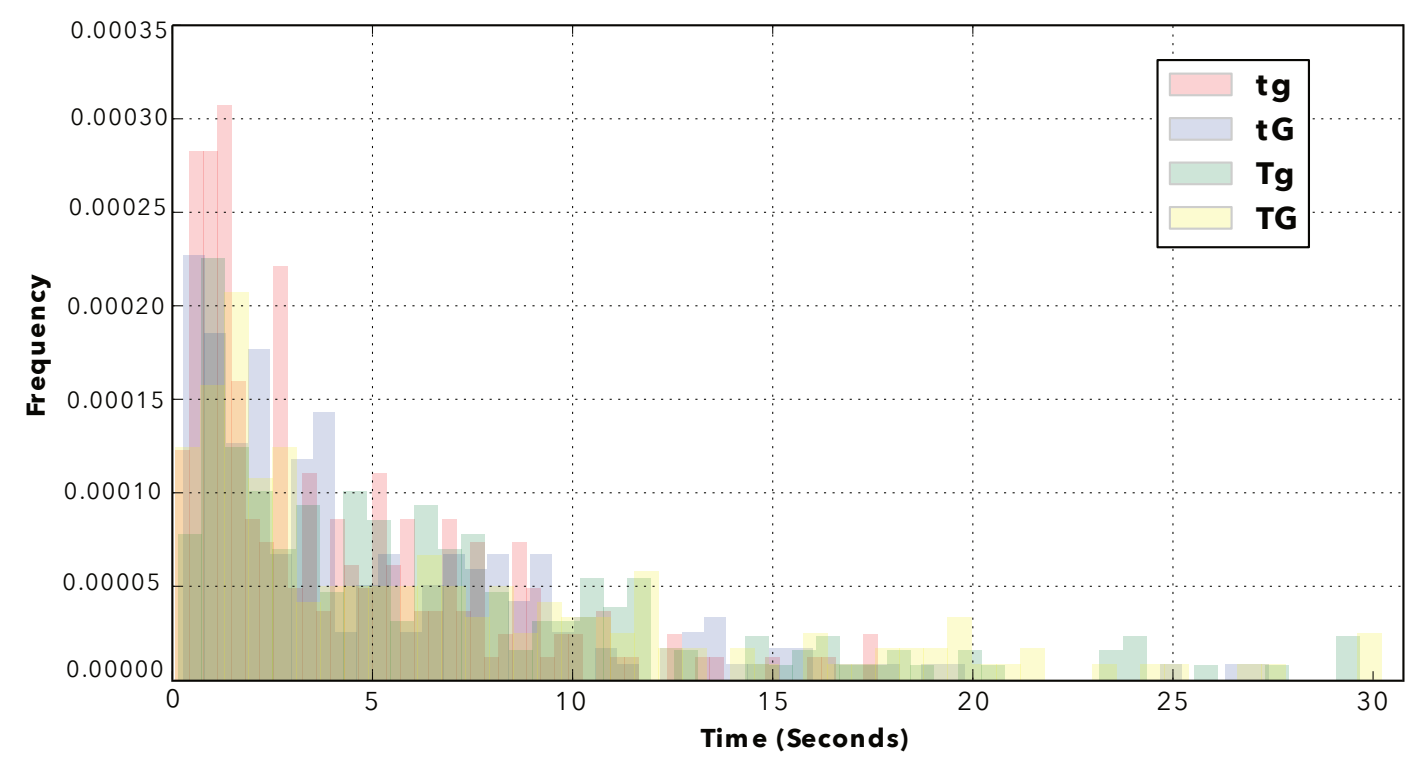

Figure 10: Glances per condition for the visual complexity experiment. In total, we observed a total of 869 engagements with the handheld display across all conditions, which ranged from less than one second (likely checking if new content has updated) up to 30 seconds in the more complex conditions.

ant effect on the participants' time engaged with the content $(\mathrm{F}(3,17)=14.032, \mathrm{p}<0.001)$, and post-hoc pairwise comparisons indicated there were significant differences between the complexity levels. By comparing 'tg' and 'Tg' we saw that complex text had a significant effect on engagement ( $\mathrm{p}=0.001)$, and by comparing ' $\mathrm{tg}$ ' and ' $\mathrm{t} G$ ' we also can see that graphical information had a significant effect $(p=0.015)$. Moreover, combined complexity of graphics and textual information also had a significant effect on engagement $(p=0.001)$.

The average duration of a glance to the second screen for the participants was $4.6 \mathrm{~s}$ for ' $\mathrm{tg}$ ', 7.1s for ' $\mathrm{Tg}$ ', 6s for ' $\mathrm{tG}$ ' and $8.4 \mathrm{~s}$ for ' $\mathrm{TG}$ ' - textually complex pieces of content yielded the lengthiest gazes. Further, there was a much larger spread in the length of glances for textual information, for example standard deviations for ' $\mathrm{tg}$ ' 


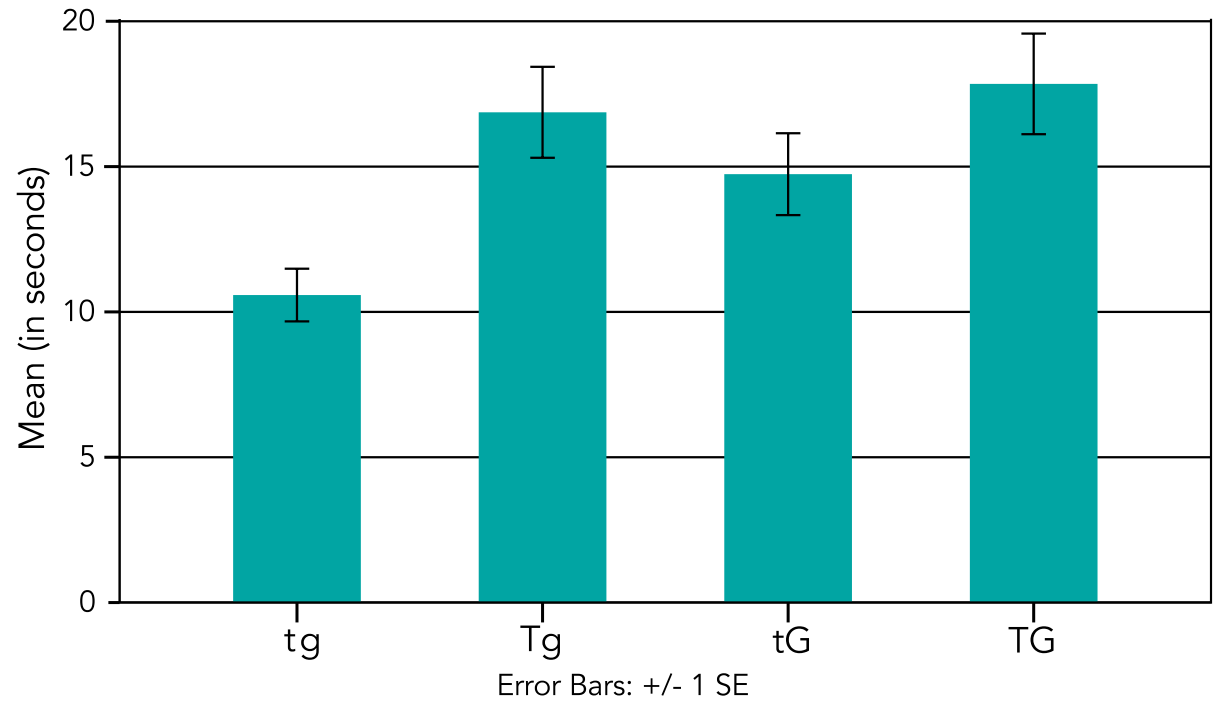

Figure 11: Length of time, in seconds, that the participants were engaging with the content for the respective complexity types.

$(\mathrm{SD}=2.46)$ and ' $\mathrm{tG}$ ' $(\mathrm{SD}=2.50)$ were much lower than the glances for the more textually complex cases - for 'Tg' $\mathrm{SD}=4.67$ and for 'TG' $(\mathrm{SD}=4.39)$. There was a significant effect for visual complexity on average gaze length $(\mathrm{F}(3,17)=5.186, \mathrm{p}=0.010)$ and post-hoc analysis showed a statistically significant difference $(\mathrm{p}=0.013)$ between complexity conditions 'tg' and 'TG'. However, we found no significant differences for complexity when considering glance frequency. Upon conducting a two-way ANOVA to assess if there was an interaction effect for textual and graphical complexity with regards to time engaged with the content, there was no significant interaction effect, implying that the complexity types (textual and graphical) do not affect each other. 


\subsubsection{Experimental Questionnaires}

Now turning to the post-clip impression questionnaires, as summarised in Table 4. There was a significant overall effect for Statement 2, the extent to which participants found it challenging to take in content on the tablet. Post-hoc analysis indicates that complex text and graphics ('TG') was ranked significantly higher than simple text and graphics (' $\operatorname{tg}$ ') $(\mathrm{Z}=2.665, \mathrm{p}=0.032)$. When we consider how much the participants believed they felt they were missing material on the tablet (Statement 4) there was a significant overall effect for complexity. In the post-hoc analysis, 'TG' was significantly more impacting than 'tg' $(Z=3.27, \mathrm{p}=0.004)$ and ' $\mathrm{tG}^{\prime}(\mathrm{Z}=2.56, \mathrm{p}=0.036)$, and that ' $\mathrm{Tg}$ ' was rated significantly higher than ' $\mathrm{tg}$ ' $(Z=2.48, p=0.026)$.

\begin{tabular}{|c|c|c|c|c|c|c|}
\hline \multirow[b]{2}{*}{ Statement } & \multicolumn{4}{|c|}{ Mean Rank } & \multirow[b]{2}{*}{$x^{2}$} & \multirow[b]{2}{*}{$\mathrm{p}$} \\
\hline & 'tg' & 'Tg' & 'tG' & 'TG' & & \\
\hline S1) I found it challenging to take in the content on the TV & 2.33 & 2.33 & 2.43 & 2.93 & 3.78 & 0.290 \\
\hline S2) I found it challenging to take in the content on the tablet & 2.03 & 2.73 & 2.30 & 2.95 & 7.93 & 0.047 \\
\hline S3) I felt like I was missing material on the TV & 2.20 & 2.48 & 2.40 & 2.93 & 3.86 & 0.277 \\
\hline $\left.\mathbf{S}_{4}\right)$ I felt like I was missing material on the tablet & 1.83 & 2.63 & 2.33 & 3.2 & 16.44 & 0.001 \\
\hline $\left.\mathbf{S}_{5}\right)$ I could easily take in the picture based material & $3 \cdot 13$ & 2.43 & 2.40 & 2.05 & 9.19 & 0.027 \\
\hline S6) I felt I could easily take in the textual content & 3.13 & 2.11 & 2.61 & 2.16 & 10.72 & 0.011 \\
\hline $\left.\mathbf{S}_{7}\right)$ Rate, out of 10 , the level of complexity on the tablet & 1.55 & 3.03 & 2.18 & 3.75 & $24 \cdot 35$ & 0.000 \\
\hline
\end{tabular}

Table 4: This table shows the statements participants were asked to consider after each clip. They ranked all statements on a 5 point Likert scale (Strongly Disagree (1) up to Strongly Agree (5)). Groups with statistically significant inter-condition effects are denoted with an asterix, therefore we can see that there was no significant effect found for Statements 1 and 3.

For picture based materials (Statement 5 ) there was a significant overall effect, however, upon conducting post-hoc analysis significant pairwise differences between conditions were not found. Analysis of participants' responses to taking in tex- 
tual content (Statement 6) showed a significant overall effect, as shown in Table 4. Post-hoc analysis indicated that 'Tg' that was rated significantly lower than 'tg' in terms of textual comprehension $(Z=3.31, p=0.004)$, this was also the case for 'TG' $(Z=2.642, \mathrm{p}=0.024)$. When asked to rate their perceived complexity on the tablet out of ten (Statement 7 ).

There were significant differences between conditions. Post-hoc analysis indicates that there were significant differences between 'TG' and ' $\operatorname{tg}$ ' $(\mathrm{Z}=3.53, \mathrm{p}$ $<0.001)$, between ' $\mathrm{Tg}$ and ' $\mathrm{tg}$ ' $(\mathrm{Z}=3.49, \mathrm{p}<0.001)$, and between ' $\mathrm{tG}$ ' and ' $\mathrm{tg}$ ' $(\mathrm{Z}=2.37, \mathrm{p}=0.018)$. The post-study questionnaire suggested, in general, that the participants found less detail (median rating of 3 ) better than more detail (median rating of 4). Finally, they generally agreed that it was hard to take in both screens at once - (median rating of 2 (disagree)) to the statement 'I found it easy to take in the both screens at once".

\subsubsection{Post-study Interview}

When questioned about what the more detailed content did to their viewing habits across both screens, the most common response was that they prioritised the TV (8 participants). That is to say, sometimes they ignored the more complicated companion content to engage with the TV. Generally, they noted this was because they were engaged with interesting things happening on the TV. For example, a tension point in Clip 4-"I found myself missing material because of the tensions in knowing if the person was going to get it [...] it was a visual thing, rather than just listening to see if they were going to get it right" $\left(\mathrm{P}_{13}\right)$, or when the presenter of CLIP 1 was giving a detailed lecture. 
The participant's comments indicated that detailed textual information was a major issue to take in at points. A common trend for users was monitoring the TV with their auditory channel and looking up when it piqued their interest - a pattern also evident in our interviews and online questionnaires in the previous chapter. However, what we learn from this study is that increasing the textual complexity affects this considerably. During the experiment, 12 participants noted that they had trouble taking in the textual information while engaging with the TV -"There was too much text and I wouldn't take it on if I was reading it, so I would rather watch the television" $\left(\mathrm{P}_{4}\right)$.

In addition, it was clear that in portions of a clip which contained more verbose passages from the programme's presenter, combined with in-depth textual information on the companion device resulted in considerable problems for the participants "It was very hard for me to read [...] while there is a TV narrative going on" (P8). To a lesser extent, more detailed pictorial information was perceived as an issue. Three participants noted that they found it hard to take in some of the map and graph-based information.

An interesting viewing behaviour was that half of the participants (10) appeared to be viewing the content on the tablet by anticipating logical gaps, or 'cues' in the TV content. It was clear that they associated certain points in the programme with cues to look at the companion device - points of low inactivity (a player mulling his shot in snooker), large panoramic shots, and repeated parts (such as replays), for example -"So there were periods on the first one [CLIP 1], where the guy was talking but he was walking across a field so it did not require my visual attention. So I could look at the tablet and still listen to it” (P6). 
Conversely, they associated other sections with points where they should look away from the tablet and focus on the TV. These were generally tension points, for example, a question being asked in a quiz programme, excitement in the commentator's voice, or even points with unexplained silences - "[...] with the snooker the audience would gasp, or when I could hear commentators go quiet I could tell something was about to happen" (P2o). Moreover, it was clear that those with knowledge or experience of a programme were more likely to anticipate these gaps better, and respond to second screen content accordingly. For example, one participant - a keen fan of snooker - was able to anticipate when certain snooker players were likely to take their shots, and adapted their viewing accordingly -"Ronnie O'Sullivan is quick with his shots and you know that you're going to miss it if you are not attending" $\left(\mathrm{P}_{13}\right)$.

Our objective and subjective evidence indicates that textual information has a significant impact on the dual-screen experience. We suggest that this is because graphical information can easily give us a gist, whereas to gain information from text we must systematically read line by line. This is supported by the fact that the average gaze length of the participants was significantly longer for the more textually complex information, and not for the graphical. We also saw larger variations between the length of gazes for the textual content compared to the graphical. A potential explanation is offered by the psychology literature is that people are poor at reading text and taking in auditory information concurrently [123]. Therefore, our participants would have had to focus considerably more on the text and 'block out' the TV in the background, due to the burden on cognitive load. 
Our findings around the impact of increasing textual complexity support the objective findings of Kallenbach et al. [74], in which participants felt more stress while watching TV and reading text (their study, in contrast to ours, displayed the text on the same screen as the programme). In the worst case scenarios, with complicated streams of auditory information on the TV, and complex tablet textual information, the participants mostly switched to the TV - the more prominent, motion rich display, with an additional auditory element, as in Brown et al.'s work [23]. In general our gaze durations were proportionally in line with other studies $[23,66]$, but slightly higher - likely related to each piece of content's lower presentation time.

Though textual information was clearly a major factor, the perceived complexity of the content was also heavily affected by the visual and auditory interaction of the larger TV content stream. This was driven by several, more nuanced, factors. Interestingly, these were also sometimes driven by some kind of prior knowledge, such as knowing how good a snooker player is. What this may suggest is that a familiarity with certain TV content means that we can learn to better manage our attention resources to accommodate second screen activity. For example, in more repetitive programming, with a limited set of visual events, this may allow a user to better monitor the audio feed from the TV to get a subconscious gist of the visual content.

We also noted two main user perceptions of content that are unrelated to that of the main TV programme. In general they supported Valuch et al.'s [136] proposal of similar elements between displays making it easier for users to switch between them. However, in addition, we found that our participants belonged to two main categories: 
- Unexpectedly, those who felt it was easier to take in the content because it was unrelated, and therefore they could compartmentalise one piece of content and fully engage with another; and

- those who found it distracting to deal with two very disparate pieces of information - a potential explanation for the findings of Van Cauwenberge et al. [137], who noted no significant effect for the relatedness between screens when dual-screening.

Though the TV display's complexity is highly nuanced and subjective, some common themes came from our research that suggested the participants would find it challenging to view second screen content during these points. The characteristics of complex TV content, i.e., a broad set of heuristics to consider television's complexity by were:

- intense dialogue - five out of 20 participants noted that they found the points where intense dialogue was occurring to require excessive cognitive processing;

- novel occurrences - six out of 20 participants noted that when something totally new happens on the screen, for example a scene switch, they found it hard to take in the companion content; and

- semantically vital visual information - for example, when someone draws something in a description and we cannot infer what it is from the auditory channel (eight out of 20 participants). 
In general, our findings suggest that we need to actively consider the complexity of information on second screens when providing complementary material to primary media. Moreover, before designing content, we should examine the potential effects of the complex interaction between the two displays, across the auditory and visual domain.

In this chapter we have explored the effect of varying textual and graphical complexity on a dual-screen experience. We have noted profound effects for increased textual, and to a lesser extent, graphical complexity. In terms of answering RQ1, which seeks to understand the main bottlenecks of the use case, we found that by increasing textual complexity we significantly increase the cognitive effort required to engage with two screens. In addition, our qualitative interview data also suggest key themes in television complexity which may inform designers when second screen content should be provided, and at which level of complexity.

The findings in this chapter have allowed us to quantify, in terms of standardised metrics, the impact of second screen content, expanding on the data gained in our online questionnaires and interviews, where we captured more broad, qualitative data. Additionally, our findings in this chapter around the effect of increased textual complexity may explain the fact that, in our previous study, users reported higher mental effort when searching for information - which likely involves more verbose textual descriptions, as opposed to brief social media content and messages. Our findings equip application designers with a sense the boundaries of perception when designing applications in terms of the complexity of visual content in relation to given TV content. In the next chapter we extend this work 
to consider how such findings may be used to better design the complexity of a cross-device media experience, and explore the effects empirically. 


\section{CHAPTER SIX}

\section{Compensating for Cross-Device Complexity}

In the previous two chapters, we have considered what types of content affect cross-device attention in a dual-screen scenario and tightened our focus to consider the driving factors behind a user's attention distribution. We found that textual complexity on a second screen, and the specific nuances of a programme, have a major impact on the user's experienced mental effort when second screening. From this, we have established a strong sense of what constitutes 'complex material' when intermixing a television programme and second screen material. Although such heuristics may allow designers to consider more deeply the implications of introducing content to the users, they do not fully consider how this should be implemented.

In this chapter, we describe our first intervention and aim to shed light on RQ3 (how can we effectively intervene in user attention to improve the way they manage it?). We consider the effect of automatically adjusting visual complexity on a second screen tablet around a set of heuristics, which we term complexity curation. Building on the findings of the previous chapter, we design an intervention which allows for the automatic variation of the second screen (textual) complexity in accordance with our heuristics, comparing this to a random baseline. Further, towards exploring $\mathrm{RQ}_{4}$, we aim to investigate the extent to which the user is in control of the way their attention is managed by allowing users to adapt 
the complexity themselves, and explore the tradeoff between interaction cost and autonomy.

We first we contextualise our techniques in the literature on adaptive and adaptable interfaces, proceeding to describe our methods for complexity curation. Then, we study the experiential effects of complexity adjustment and adaption with users. Finally, we conclude with our design recommendations and implications from our findings.

\subsection{ADAPTIVE AND ADAPTABLE UI}

Much work in HCI for TV and HCI in general looks at adaptive and adaptable interfaces. Often, there are scenarios in which we want to provide a user with the exact amount of content they require - no more; no less. Too much information often overburdens a user cognitively, whereas too little is insufficient. Findlater and McGrenere [45] term this class of changing interfaces as follows: adaptive - the systems controls change; adaptable - the user controls change, it is customisable. They term interfaces which do not change, and are unchangeable, as static. In their work comparing static, adaptive and adaptable menus, they explore the perceived efficacy of each, finding that static menus were significantly faster than adaptive, and that in general users preferred adaptable menus overall.

Findlater and McGrenere [45], in their adaptive condition, used an algorithm that dynamically determined which items should appear in the upper partition of a menu based on the user's most frequently and recently used items. In later work [46] they focus on applying similar techniques to solving the problem of perform- 
ing tasks on smaller interfaces, where showing all the information available is not easily possible.

As computing devices' ability to sense and engage with their environments has increased, much recent work has extended adaptive UI by designing attention aware systems. Attention aware systems work by gauging the attention of the user, and acting appropriately. Such interfaces generally take physiological data from the user and make assumptions about their needs. These interfaces, termed physiologically attentive user interfaces [26] take features such as heart rate variability (HRV), electroencephalogram (EEG) information and eye tracking (c.f. [69]) to approximate attention in users and adapt accordingly.

\subsection{ADAPTIVE AND ADAPTABLE UI: TV FOR HCI APPROACHES}

Now, turning to the focus of this thesis - UX for dual screen television - there are numerous reasons for gauging a user's television viewing habits. Knowing information, such as what types of television users watch or how often they watch it, enables broadcasters to recommend appropriate content. Various methods can be used to infer such habits, and now with television being increasingly internetbased, we can infer more and customise the content with even finer detail so that users may have a totally unique experience.

\subsubsection{Recommender Systems for TV}

Recommender systems were borne from the inception of digital TV's diversity of choice. Due to the vast number of digital channels available, users found themselves getting lost in irrelevant media. One solution, as described by Isobe et al. 
[68], is to allow a user to provide some information about themselves, such as how long they watch television per day, or their viewing interests, and then recommend content accordingly. This is, perhaps, analagous to an adaptable UI, where users control change. Since then, there have been numerous variations on the theme of content recommendation. Bernhaupt et al. investigated this from an ethnographic standpoint [14], noting that such systems only really work on a wholly individual level, due to great interpersonal differences in preferences. There are a number of important factors to consider when recommending television content, and extensive user modeling has aided in generating realistic profiles for users, as in the work of Shin et al. [128] and Oh et al. [108] - which could be interpreted as adaptive UIs, where the system controls change based on its inferences from the user.

\subsubsection{Perceptive Media}

Perceptive media, arguably a form of an adaptive UI, takes customising content to the next level by embedding related information within a media experience on a user-by-user basis. It relies on using data we know about a user to not just recommend specific shows, but adapt them to their needs. The information is normally inferred implicitly [48], that is, the user does not manually input information about themselves, but it is sensed through their browser, or operating system. For example, their location ${ }^{1}$ or their social networks ${ }^{2}$.

1 Perceptive radio play - http://wWw. futurebroadcasts.com/

2 Interactive short movie based around your Facebook feed - takethislollipop.com 
As discussed in the previous section, there is much work around adapting the complexity of systems and webpages, and a plethora of systems which recommend television programmes and material based on users' choices and devices' inferences. Further, as shown in the previous two chapters, there is a evidently a large cognitive effect on increasing the complexity of second screen material, especially in tandem with more demanding television content. In the remainder of this chapter we re-appropriate techniques from the from the fields of adaptive and adaptable computing towards compensating for the perceived complexity of cross-device media.

As we, in the previous chapter, garnered an impression of the characteristics of complex television content, we can begin to consider methods by which application designers and broadcasters may compensate for primary screen complexity when developing their companion applications. Indeed, a reasonable solution to this issue may be to not introduce visually complex second screen information during complex points in a programme. However, this is clearly restrictive, and means we cannot provide the time-relevant second screen content that the literature suggests is salient.

Towards a deeper understanding of $\mathrm{RQ}_{4}$, which seeks to investigate how in control of attention management a user should be, we now explore two techniques with a view to understand ways by which we may adapt complexity on a second screen to compensate for the perceived complexity on the primary: 
- Complexity Curation - similar to adaptive user interfaces, the device adapts its content's complexity in accordance with the television's perceived complexity (low user autonomy);

- Complexity Adaption - similar to adaptable user interfaces, we give the users the ability to adapt the complexity, up and down, themselves (high user autonomy).

We now describe the our content manipulations in detail, along with an extensive empirical study to understand their subjective and objective effect.

\subsubsection{Device-Driven Complexity Curation}

To compensate for dual-screen visual complexity we propose that content can be curated - rendering the content more visually simple on the handheld device when more complex content is on the TV, and more complex when simpler content is on the television (as in Figure 12). When new content is loaded by the user, the view's complexity can update based on the characteristics of the TV content.

For our complexity curation method, we divide the content into appropriate time segments. Here we have chosen 20 seconds because this was the approximate time between scene/shot changes. Then for each of these segments we consider the TV complexity characteristics, discussed in Section 5.4. If the content contains none of the characteristics of complex TV content, then we consider it the lowest level (1), and if it contains them over the majority of the material we rank it the highest level (3). If the segment contains a mixture of complex and simple scenes, we consider this a medium (2) complexity. 
a)

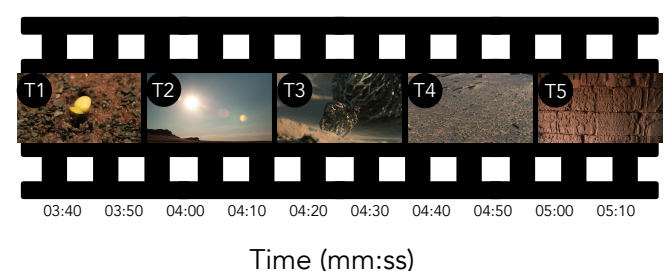

b)

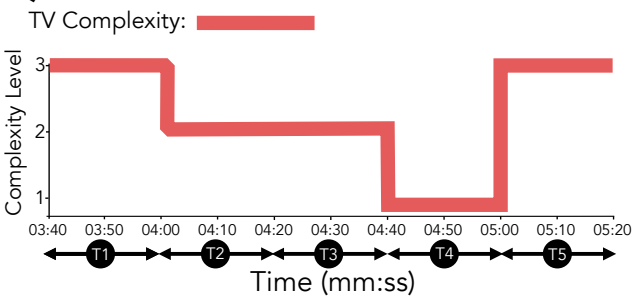

c)

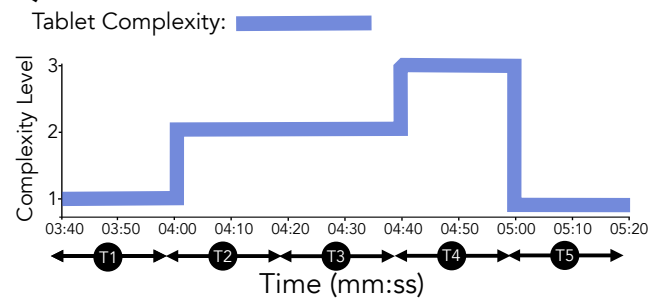

Figure 12: Content curation process: a) video content is broken down into 20 second time slices; b) complexity of TV content is determined, for example by our TV complexity characteristics; c) complexity of tablet content is determined by inverting the TV complexity value to compensate for the complexity across the foci. For example simple TV (complexity level 1) means tablet complexity would be adjusted to complexity level 3 .

\subsubsection{Complexity Adaption by Users}

To compensate for complexity, another feasible option is to load the material in a default level of detail, and allow the user to adapt the content themselves (see Figure 13). This can be facilitated with a 'more/less' info button, which increases the information in a given text region, in a similar approach to Geerts et al. [53]. Therefore, if a user wants to find out extra information, they can expand the text. Conversely, by giving them the opportunity to turn the complexity down, we also aimed to discover if users compensate for visual overload themselves with our methods, as they were shown to do in their own viewing (as determined from our interviews in Chapter 4 ). 


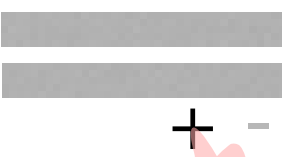

a)

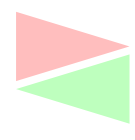

(1)

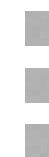

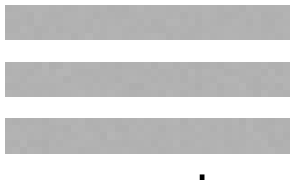

\section{b)}

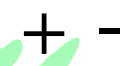

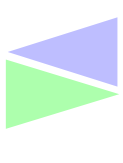

)

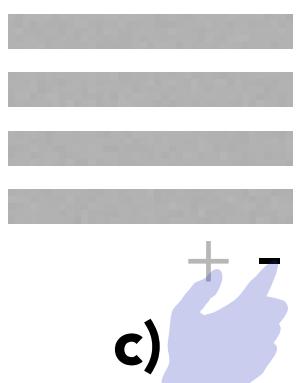

c)

Figure 13: UI Adaptation: the user can alter the complexity and amount of information in a text element. The arrows correlate to the actions possible by the user, for example in b) it is possible to increase, or decrease complexity, and in a) the user can only increase from the simplest state.

Between these two methodologies, we predominantly aim to gain insight into RQ4 - will affording the user high/low autonomy have any perceived benefits? And also, gain insight into the overarching question of $\mathrm{RQ}_{3}$ - whether we can effectively intervene in a user's attention by varying the complexity of the content.

\subsection{EMPIRICAL STUDY OF COMPLEXITY COMPENSATION}

In this study we explore the impact of complexity curation UI, and user-adaptable UI, compared to a baseline context unaware case. We do so with an aim to determine if complexity curation is a useful starting point to balance for complexity across dual-screens (which is crucial to cross-device experiences, as outlined in the previous chapter); to further validate our complexity characteristics; and to examine the relative user experiences of both of the adjustment methods. 


\subsubsection{Procedure}

We conducted this experiment in a TV usability lab and were able to capture video of the participants from multiple angles in a mock living room. First we briefed the participants. We informed them that there were three conditions, which we explained in simple terms, and ensured they understood. Not to bias the experiment, we did not tell them the order of the conditions - allowing us to compare the curation to the randomized case. After gathering participant demographics, the participants then watched three clips, with a piece of companion content on a second screen (iPad). Ordering effect for the variables was counterbalanced by a Latin square design - over all participants, each clip was used with the same number of independent variables (complexity levels). After each clip, we administered a short questionnaire to evaluate their experience. After all clips had been watched, we administered a post-study questionnaire to evaluate the participants' experience as whole. A full questionnaire can be found in Appendix C. After this, we concluded with a brief semi-structured interview.

\subsubsection{Stimuli and Tablet Content Design}

The participants were presented with a navigable application (Figure 14), in which they could view content related to the TV programme. This refreshed with new information at four points in the programme. This was indicated by a simple auditory notification and a new piece of content appearing in the navigation bar. Participants were free to return to previously visited content, which would render differently based on the condition active. 


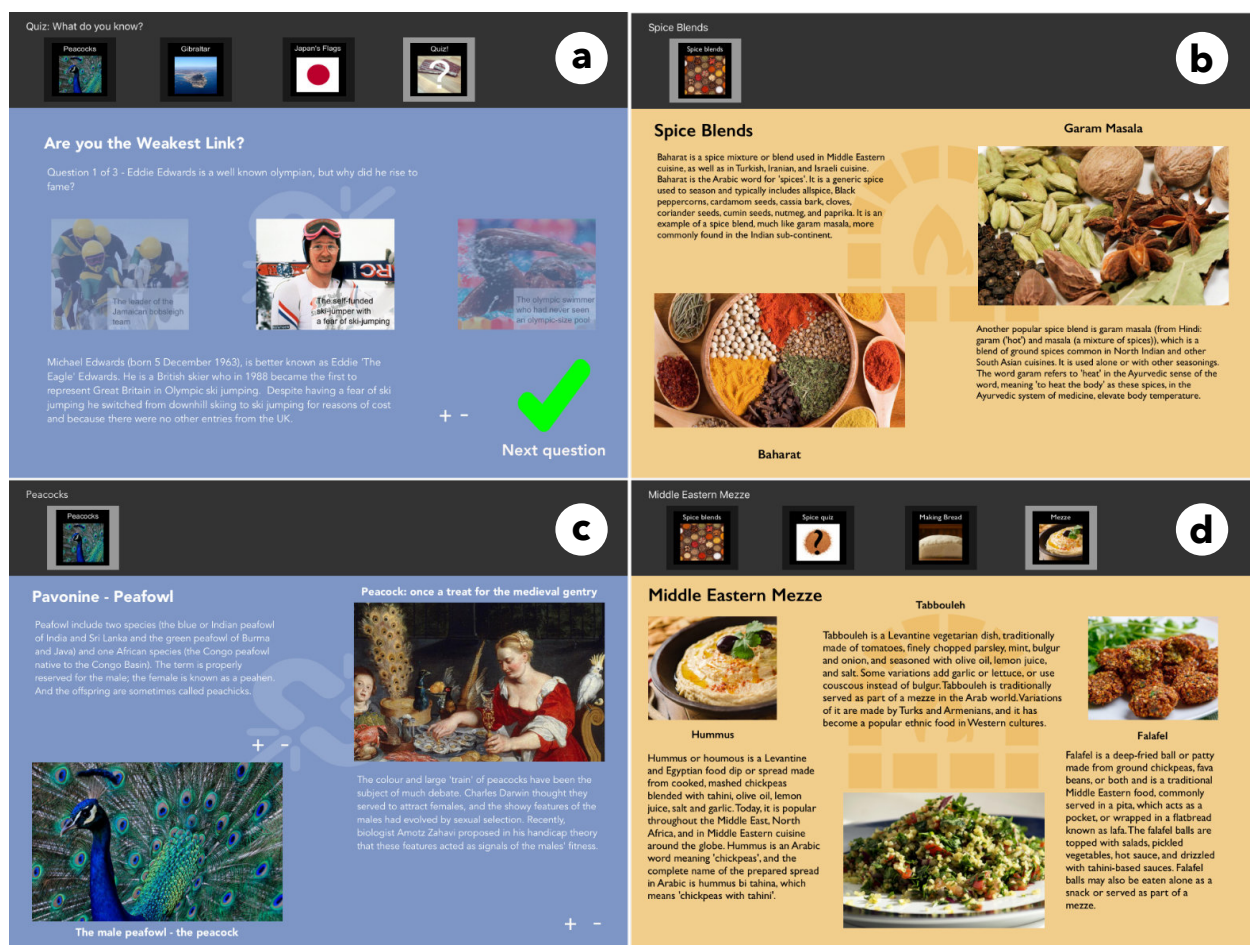

Figure 14: Four examples of screens from a navigable application designed to support Clip 2 (a)) and Clip 3 (c)). The two left clips depict the condition in which the users can adapt the complexity, and a) and d) where it is curated for them (discussed in the coming section).

The participants were motivated to engage with the material on the tablet through a quiz in which they were asked three questions on the companion application about the content on the tablet and/or the TV programme. As in the experiment in the previous chapter, informed by our interviews and online questionnaires in Chapter 4 , we chose the stimuli to represent a diverse set of complexities, but now informed by our additional findings as to what constitutes complex TV material. Each clip was approximately 7 minutes and taken from a large TV broadcast archive. The clips used can be viewed in Table 5 . 
We chose CLIP 1 as it fulfilled the requirements for both complex, and simple TV content, with both intense dialogue and often scarce imagery. We chose CLIP 2 as it fulfilled requirements for complex content, as it is full of tension points and intense dialogue. Finally, we chose cLIP 3 as it fulfilled the characteristics of simple TV content, with relatively little complex dialogue.

\begin{tabular}{lll}
\hline Clip No. & TV Programme & Summary \\
\hline 1 & Africa: Sahara & Natural history documentary \\
2 & The Weakest Link & Quick-fire quiz programme \\
3 & James Martin's Home Comforts & Cooking programme \\
\hline
\end{tabular}

Table 5: Clips used in the complexity adjustment experiment.

To consider how we may accommodate for complexity, we defined three second screen visual complexity levels informed by the findings of the previous chapter: SIMPLE, in which we used a reading score of 70 (Flesch-Kincaid) and a word count of 40; MEDIUM, in which we used a reading score of 55 and a word count of 55 ; and COMPLEX, in which we used a reading score of 50 and a word count of 70 . Some examples of text, which we adapted from online articles ${ }^{3}$, included in the applications is shown below:

- Simple: A superior mirage is one that is displaced above the horizon. For example, while at sea we may see a ship above its physical position on the ocean.

- Medium: A superior mirage is one that is displaced above the horizon - while at sea we may see a ship above its physical position. They occur when the air below the line of sight is colder than the air above it. The image shifts up as air above cold air is the opposite of the normal temperature. 
- Complex: A superior mirage is one that is displaced above the horizon. For example, while at sea we may see a ship above its physical position. Superior mirages generally occur when the air below the line of sight is colder than the air above it. This unusual arrangement is called a temperature inversion, since warm air above cold air is the opposite of the normal temperature gradient of the atmosphere. Passing through the temperature inversion, the light rays are bent down, and so the image appears above the true object, hence the name superior.

These textual complexity levels, as discussed in the previous chapter, are typical of supplemental information-based companion apps. In terms of implementation, we stored each piece of textual stimuli in memory in the application and presented it depending on the appropriate complexity at the given time (as shown in Figure 12), leaving enough space in the rest of the UI to accomodate for the largest size (COMPLEX). The independent variable in this experiment was how we controlled for this complexity. We looked into two methods (adaptable and curated), compared to a baseline, to uncover the implications of each:

- Context unAware (Condition 1): second screen material that updates without any consideration for first screen complexity - it is randomly generated across three levels of complexity - SIMPLE, MEDiUm and COMPLEx. This is done each time a new view is loaded by the participant while browsing the application.

- Curated (Condition 2): second screen content that is curated to TV content. We curated this in accordance with our complexity curation methods discussed in the previous section. The content was rendered to a new condition 
each time the participant opened a new view. Each level of complexity was used the same number of times for curation across the clips to ensure a fair comparison to Condition 1.

- ADAPTABle (Condition 3): content that the users can make more or less complex by interacting with the interface. The content loaded in MEDIUM complexity, and then the participants could adapt it, as discussed in Figure 13.

\subsubsection{Participants}

With regards to the participants, we recruited students and staff from Salford University, as well as people from BBC, which were not related to this research. For example, administrative staff and software developers. Of our participants, 14 identified as male, and 10 female, making for a total of 24 participants, with ages ranged from 21 to 52 , with a mean age of $32.3(\mathrm{SD}=9.75)$. With regards to their TV/technology experience, 19 participants (79\%) reported engaging with their devices while watching TV. On average, the participants consumed around 2 and a half hours of video media per day, and (38\%) had engaged with some form of companion application (such as Zeebox, or 'The Voice' Companion apps). All bar one of the participants were native English speakers.

\subsubsection{Measures and Validation Techniques}

For the post-clip Likert questions, we asked how the detail on the tablet affected their dual-screen experience. We were specifically looking to investigate the three conditions in terms of their advantages and/or disadvantages for dual-screen UX, the baseline (CONTEXT UNAWARE) acting as a reference to compare to. We looked 
at whether the adjustment type had an effect on the participants' ability to take in either the TV, the tablet, or both screens better than the baseline.

To quantify our findings, we logged interaction data for when the participants navigated between new views, at what complexity level the views loaded up, what condition they were assigned, and how much they adapted the content themselves (up or down). To conclude the experiment, we conducted a brief semi-structured interview with two main questions: one that probed if they had noticed that the content on the tablet had adjusted to the complexity of the TV for any of the conditions; and another in which we asked how they felt about interacting with the buttons to change the complexity on the tablet.

\section{$6.5 \quad$ RESULTS}

We now describe the results, beginning with the questionnaire data, progressing to explore the device logs, and finally, considering the interview questions.

\subsubsection{Questionnaire Data}

Concerning inter-condition effects, post-clip Likert scale results (Table 6) indicate that there was a significant difference between the conditions for the participants taking in information on both screens simultaneously. Post-hoc analysis determined that they agreed that Condition 2 (CURATED) allowed them to take in both visual streams significantly better than the CONTEXT UNAWARE case $(Z=2.07$, $\mathrm{p}=0.038)$, and also the ADAPTABLE $(Z=2.72, \mathrm{p}=0.009)$ technique. However, there was no significant difference between the CONTEXT UNAWARE and the ADAPTABLE condition. 


\begin{tabular}{lllllllll}
\hline & \multicolumn{3}{c}{ Mean Rank } & & & \\
\cline { 2 - 3 } \multicolumn{1}{c}{ Statement } & $\mathrm{C}_{1}$ & $\mathrm{C}_{2}$ & $\mathrm{C}_{3}$ & & $\chi^{2}$ & $\mathrm{p}$ \\
\hline 1) The tablet detail allowed me to take in tablet content & 1.88 & 2.29 & 1.83 & 4.48 & 0.11 \\
2) The tablet detail allowed me to take in TV content & 1.85 & 2.31 & 1.83 & 4.33 & 0.12 \\
3) The tablet detail allowed me to pay attention to both displays & 1.85 & 2.46 & 1.69 & 9.72 & 0.008 \\
\hline
\end{tabular}

Table 6: Participants were asked to state their agreement after each clip. All questions were ranked on a 5 point Likert scale. Groups with statistically significant inter-condition differences are denoted in red and darkness of colour denotes strength of agreement.

Turning now to the post-study Likert scale data, it was evident that there was a significant effect for condition on the amount that the participants felt that the " $d e$ tail of the tablet content enhanced the experience as a whole" $\left(\chi^{2}=7.59, \mathrm{p}=0.021\right)$. Post-hoc analysis suggested that participants rated Conditon 2 (CURATED) significantly higher than CONTEXT UnAwARE $(Z=2.28, p=0.039)$. However we found no statistically significant difference between CURATED and ADAPTABLE. With regards to the demographics data, there was a significant correlation between those participants who stated they were easily distracted by content on their devices and those who enjoyed the curated case (Spearman's $\rho=0.40, p=0.027$ ).

Although the method in which the content's complexity was adjusted did not significantly differ between clips, the participants rated these clips significantly differently for their general preferences. A significant overall effect was noted for the amount they enjoyed the clip $\left(\chi^{2}=13.78, \mathrm{p}=0.001\right)$. We conducted post-hoc analysis and found that CLIP 2, The Weakest Link, was ranked significantly lower than CLIP 1 (Sahara) $(Z=2.57, p=0.015)$ and CLIP 3 (Home Comforts $)(Z=2.55, p=0.008)$. 


\subsubsection{Interaction Data}

In total, we logged 216 complexity adjustments (in the adaptable case) across all clips. In general CLIP 2 yielded the most adjustment, with a total of 92 (3.8 adjustments per clip), followed by CLIP 1 at 64 (2.7 adjustments per clip) and lastly CLIP 3 at 60 (2.5 adjustments per clip). In both CLIP 1 and CLIP 2 turning the complexity up was more common than down - CLIP 1 saw $21.9 \%$ more complexity increase than decrease, and CLIP 2 19.6\% more. However, with CLIP 3 the participants decreased the complexity marginally (6.7\%) more than increased - summarised in Figure 15.

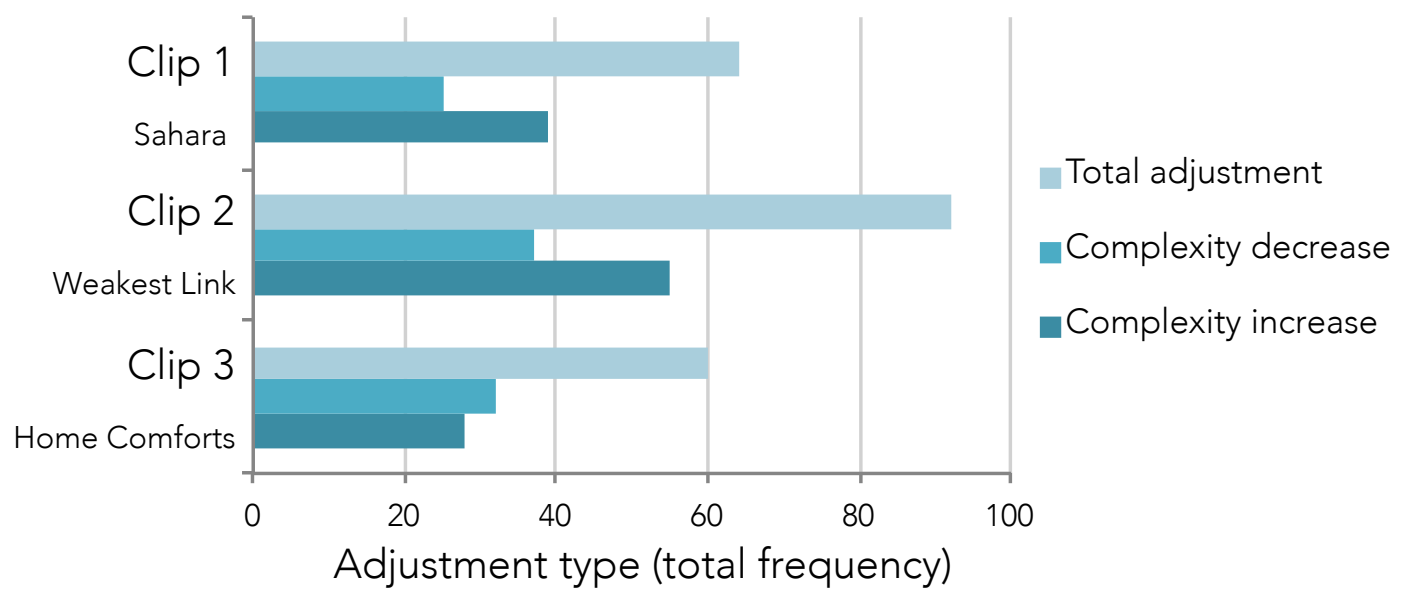

Figure 15: Frequency of participants' total complexity adjustment.

From the data logged on the device, it was clear that different clips resulted in different uses of the adaptable case. Upon being presented with a new view, we observed what the participants did first - whether they increased the complexity, turned it down, or did not interact. In CLIP 1 the most common response was for them to increase the complexity (52.0\% of the time), and a similar pattern in CLIP 2 (51.6\% of the time). However, in CLIP 3 this changed (see Figure 16), as the 
most common response was no interaction (42.8\%), and both turning up/down the complexity constituted for only $28.6 \%$ of the reaction.

Now turning to the reaction to the complexity of the TV content - as shown by the total of complexity increases, and the number of net increases in Table 7, when the TV content was of Low complexity the participants increased the tablet content complexity when loading a new view. Moreover, they were also more likely to interact in general. However, when the content was of HIGH complexity on the $\mathrm{TV}$, they were no more likely to turn the complexity down than when presented with the Medium case.

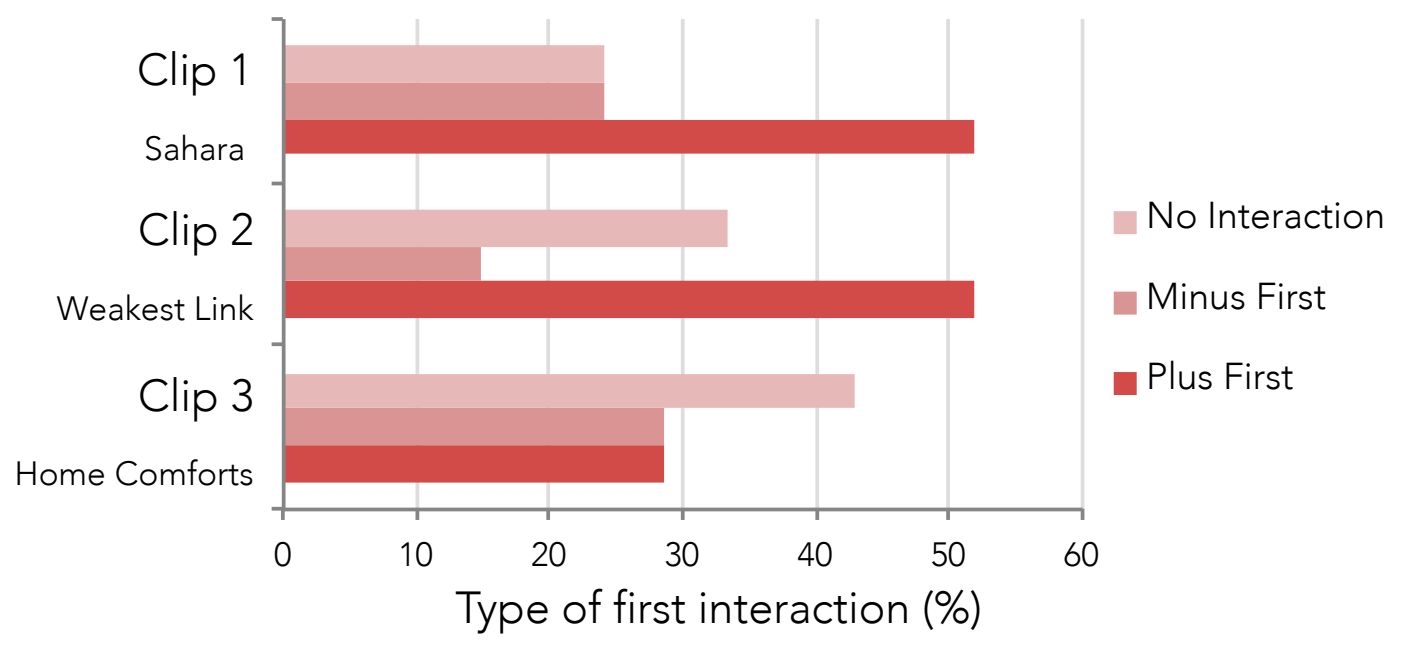

Figure 16: Frequency of participants' first adjustment choice.

\subsubsection{Post-study Interview}

The results of the interviews indicated that most of the participants did not notice the content being curated. One third (8/24) noted that they felt that the content was in some way changing depending on the TV content. Seven of these eight 
participants said, unprompted, which of the trials they felt had been adapted, with five people making a correct choice.

With regards to preferences between the methods for manipulating the content, there was a fair amount of variety. When quizzed about interacting with the UI to adapt the complexity 11 responded positively, seven negatively, and five were indifferent or did not use the function enough to say. In general, the positive responses were due to two main factors: six participants noted they liked the ability to ease off the complexity to counter complex events happening on the TV, and five noted that they liked the ability to actively select a higher complexity level. However, eight participants noted that the cost of interaction with the adaptive UI was detracting from the experience, for example $\mathrm{P}_{7}$ 's comment - "If it's curated and you don't have that control you've got a snippet of information; it's not a distraction".

The participants were not vastly overloaded by the content in CLIP 2. With regards to the TV material, comments mentioned the repetitive nature of a quiz programme; “...in the Weakest Link [CLIP 2], there's an obvious pattern - she asks the questions" ( $\left.\mathrm{P}_{1}\right)$.

\begin{tabular}{lcccc}
\hline Interaction & LOW & MEDIUM & HIGH & Total \\
\hline Up Total & 16 & 9 & 10 & 35 \\
Down Total & 5 & 6 & 7 & 18 \\
Net & 11 & 3 & 3 & 17 \\
\hline
\end{tabular}

Table 7: Frequency of first interaction when presented with a new view. The Low, MEDIUM and HIGH cases refer to the complexity on the TV 
The Likert scale data suggests that the curated case allowed the participants to take in the dual-screen experience by adjusting the tablet's textual complexity in accordance with our characteristics of TV complexity. Moreover, the adaptation data indicated that the participants were more likely to increase the complexity when fewer of the characteristics of complex TV content were presented. This tells us to some extent that the method, though a relatively simple set of heuristics, works as a good rule of thumb for considering dual-screen complexity generically.

From analysis of the interview data it was clear that, although in general the curated case was preferred, a relatively small proportion actually noticed the adaptation explicitly, likely due to its subtle nature. Several participants' comments noted that the curated content allowed for a more 'automated' experience. This, in turn, afforded a less engaged, more 'sit-back' approach (discussed by Brown et al. [23]) to second screen viewing, which many participants reported positively.

Such design choices may be made by those who wish to make 'less active' content for users to 'unwind' to, as discussed in [11]. Interestingly, a significant, and moderate correlation was found between those who got easily distracted by their devices and those who enjoyed the curated case. A potential explanation for this is that those who find it harder to manage their attention resources over the foci found the curated case helped them compensate for this.

The adaptable case often scored poorly as a method for adjusting complexity, even compared to a context unaware baseline, due to its perceived interaction cost. However, from analysis of the user comments and the interaction data we can see a more complex picture. For instance, many participants preferred the adaptable con- 
dition as it allowed them to be more inquisitive and proactive in the experience at points where little was happening on the TV, and to tone down the complexity when the TV content was taking most of their attention.

With regards to TV genre differences, the data from the interviews and the log data contradicted our expectations with regards to how much the participants would interact with the adaptable case to adapt the complexity. The data indicated that participants tended to interact more with the device during CLIP 2, likely due to the visually repetitive nature of the programme. Further, the log data suggests that for CLIP 3 they wished to take a less hands on approach to adapting the complexity, and for simply to sit back, or even turn down the complexity.

\subsection{DESIGN CONSIDERATIONS AND FURTHER WORK}

As in the previous chapter, this study suggests that we need to actively consider the complexity of information on second screens when providing complementary material to 'primary' media. With regards to the solutions discussed in this chapter, our results suggest that viable solutions are to:

- In the simplest case, not introduce content at points of high TV complexity;

- curate the content on the handheld device to adapt to the complexity on the tablet;

- provide user-adaptability to the UI; or

- a mixture of the approaches could be adopted in which the content is curated to some degree and then additional adjustments can be made to support the viewer in their individual preferences. 
Such curation can be implemented by a broadcaster, as part of the creative process when developing a tablet experience in tandem with a programme, or as a design lever to optimise viewers' distribution of attention between the two screens. Further, this could be done post-programme, for example by an independent application developer, to control the distribution of attention between the screens in key moments.

In terms of answering questions regarding the control a user should be afforded in managing their attention in such an experience (RQ4), we found that the curation afforded a better dual-screen experience in terms of taking in the content across two screens, and supported more passive 'sit back' viewing. Also, that the adaptable case allowed for the encompassment of more viewing styles, but at an interaction cost to the participants. There is an evident continuum of usage, ranging from that which affords full user autonomy, and that which affords a more automated, curated approach.

The guidelines we have provided in this chapter for curating content between the screens act as a proof of concept that content complexity can be curated with positive results. However, we note that our findings may not be fully generalisable to all use cases, and that they are most applicable to the design of informationbased companion apps (which include many contemporary apps). Moreover, the study was held in a lab with set stimuli and was therefore not subject to varying environmental factors. In addition, we only consider visual and auditory stimuli, and do not touch on more nuanced concepts such as engagement [113]. However, this chapter's findings are still cautionary for those developing more interactive UI, making it evident that complex models of what constitutes dual-screen complexity need to be formed to inform optimum dual-screen UX. 
Practically, designers may consider trialling content before public distribution and probe points in which UI is hindered by dual-screen visual overload, or drive these by user models from data physiological data such as gaze [69]. The findings of this chapter may also be broadly applied in the context of second screen apps for social media - delaying attention-impacting information on a handheld device to a point of less primary screen involvement. These could be determined by easily detectable factors such as the frequency of social media updates; allowing only the most highly ranked social media items to envelop the user's attention at key points in a live debate.

In this chapter, we have considered how techniques from adaptable and adaptive user interfaces may be utilised in the varying of second screen complexity. In doing so, we have actualised and extended the findings of the previous chapter towards overcoming the current shortcomings in the design of second screen materials.

Informed by our study and inferences from the information captured in Chapter 5, we present a novel perspective on designing second screen materials. By evidencing the clear benefits of dynamically varying second screen content, we outline the benefits and drawbacks of introducing varying degrees of user control to the way that the content changes its complexity. We have solidified our findings around RQ2 from the previous chapter in this study - varying the complexity of content has a major effect on users. Moreover, by varying its complexity, this intervention has allowed us to effectively intervene in user attention to improve the 
way that they manage it, adding to our knowledge of RQ3, which seeks to understand if we can effectively intervene in user attention.

Finally, on answering $\mathrm{RQ}_{4}$, we have documented a rich continuum of perspectives on varying the content automatically compared to allowing users to adapt it. Although, in general, a curated (low autonomy) approach was preferred, it depends highly between users. For example, we documented many cases in which the participants preferred to be in control and gather more information for insight, and others where they simply reduce the complexity for simplicity (high autonomy).

In the next chapter, we consider the research questions of this thesis in more depth by exploring a method which shifts control of the attention fully to the content designer, by exploring how one may invoke cross-device attention shifts. We explore if adding notifications into cross-device experiences, firstly: affords improved management of attention ( $\left.\mathrm{RQ}_{3}\right)$; and secondly, if providing the user with a creatormediated experience (low user autonomy) is an effective approach by embedding a diverse set of notifications into an experience to direct their attention in a variety of ways. 



\section{CHAPTER SEVEN}

\section{Mediating Attention}

In this chapter we pivot from understanding and adjusting the complexity of a cross-device experience, to consider how we may invoke attention shifts between the devices at opportune moments, providing direction and coherence to a crossdevice experience. Our previous chapters have focused on compensating for the heightened attentional resources required to engage with more than one screen. Clearly, however, there are points in which we wish a user to focus only on one device at a time. In this chapter we focus on driving user attention to converge on the desired screen, at an opportune time, so that a content creator may have increased control of user experience, for example where the user is looking at a given point.

For cross-device content, the use case is currently undirected; during a cross-device media experience the user's attention roams between the screens by whatever piques their interest. This, as discussed in the previous chapters, results in numerous attention shifts, many of which are not required and are likely to cause additional effort to engage with the experience. In time-critical scenarios, users are often unaware of material appearing on a second screen and, conversely, unaware of the events unfolding on the television when engaging with second screen content. In this chapter we explore mediating attention; that is, the notion of affecting a user's attention with additional stimuli, embedded within the experience. The aim, here is to give the creator of the material high control of the experience. In the context of the research questions, we aim to understand the general efficacy 
of intervening in attention in terms of the way the users manage their attention (RQ3) and also explore the effects of giving the user relatively low autonomy in the experience $\left(\mathrm{RQ}_{4}\right)$, i.e. telling where to look when.

The initial concept of mediating attention was motivated by the following aspirations:

- To optimise attention distribution: to ensure users are not frequently checking for updated content when not required, or missing key moments; and,

- to enhance experience production: providing minimal distraction at key points, and shifting attention to the relevant display at opportune moments, with variable and controllable urgency.

In this chapter, we initially discuss the related work around attention management in computing environments, and consider how these may be applied to the dualscreen use case. We then progress the field by chart the possibilities for attention mediation. To do so we conduct an empirical study towards understanding the impact of our techniques. Finally, we consider the implications for practitioners to affect user attention as required.

\subsection{ATTENTION MANAGEMENT WITH ADDITIONAL STIMULI}

Notifications are an everyday part of our lives - they alert us to new information, make us aware of our surroundings but, ultimately, disrupt the flow of the current activity. In the literature, they encompass all modalities and can be used to cue users, and portray varying degrees of urgency (see [143] for an investigation 
of the effect of modality on notification performance). In our everyday contact with notifications on our mobile devices, however, they tend to be one of three main modalities: auditory, visual or tactile. We focus in this work on auditory and visual methods of notification, as these are the methods most common to both the television and our mobile devices.

\subsubsection{Visual Notifications}

As previously discussed in Chapter 4 , human binocular vision and perception is highly limited in terms of its focused angular perception; that is, we view the world mostly in the peripheral. With this in mind, many computer interfaces utilise this in their design of notifications towards providing more effective and targeted alerts, depending on the situation at hand. We use state change and motion as devices for commanding attention. The frequency of a flashing light, for example, can be increased to present increasing urgency [78].

Design rationales which consider such implications are essential for bringing quick attendance to tasks at critical moments (for example, in flight decks and control rooms). Ambient awareness, such as that provided by an Ambient Display [94], can be provided by more slowly changing changes in state, allowing a user to monitor an ongoing process. Work by Magilo and Campbell [89], for instance, looked at presenting peripheral information in a ticker display, found that motion and animation should be non-continuous and minimal for peripheral awareness.

As previously discussed in Chapter 2 work on visual notifications and awareness has explored how one may design for awareness in the near peripheral, such as an Orb next to a work station [94]; or the InfoCanvas project, providing peripheral 
information [102]. Such methodologies have also been explored in $3 \mathrm{D}$ environments. Booth et al. [18], for instance, explored how a user's attention may be guided around a physical environment by varying visual cues with projections on real-world objects.

\subsubsection{Auditory Notifications}

Auditory notifications, compared to visual notifications, are useful because they are omnidirectional (i.e., one need not focus on the interface) and do not require direct contact with the user (such as tactile notifications). They do, however, also affect those around the user, leading to intrusion and related social and privacy implications (see [59] for an overview). In this chapter we utilise the two predominant types of auditory notification discussed in the literature - auditory icons and earcons.

Auditory icons, much like visual icons, aim to present iconic information about events occurring in a user interface. That is, present a relationship between the auditory information and its meaning [106]. For example, the sound of something taking off when sending an email; complementing the visual icon of a paper airplane used for the button to send the message. In general, auditory icons can be intuitive and can map well to real world occurrences; transcending language. However, they are, much like icons; skeuomorphs. Therefore, they are hindered by the fact that this mapping to the real world may not exist.

Earcons are useful, then, when we cannot easily represent an event using auditory metaphors (see Figure 17). Earcons are abstract, typically musical, sounds which aim to convey information through the notes associated structure (i.e., pitch and 

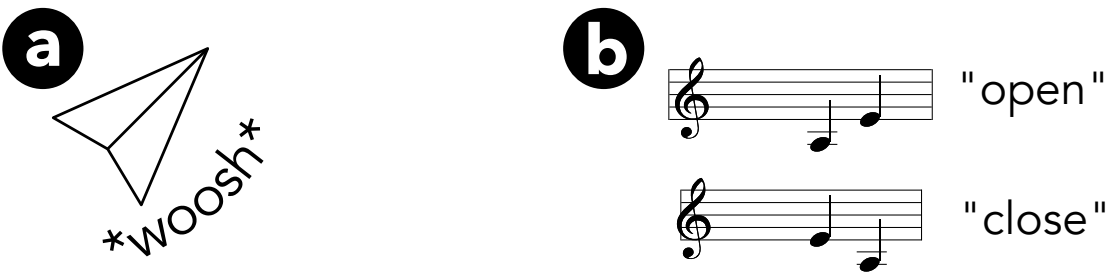

Figure 17: Auditory icons, for example the mail sending example in a), generally mimic an event happening in the real world. Earcons, on the other hand, generally aim to convey a message through abstract tones, with inherent structure. In the example in $b$ ), the top ascending sequence (an ascending perfect $5^{\text {th) }}$ denotes a file opening, then this is inverted to close the file - creating an internal logic by which the user can learn the interface.

rhythm of the note sequence). Blattner defines these as "non-verbal audio messages used in the user-computer interface to provide information to the user about some computer object, operation, or interaction" [16]. For example, opening a file may be something users inherently associate with an ascending sequence of notes; with a corresponding pitch inversion to indicate the opposite. Brewster, in early work on Earcons (1996), explored how they may be devised experimentally with users [21] to convey an underlaying structure of a computing system for notification and peripheral awareness.

In this chapter so far we have seen that there is an evident continuum of urgency that designers may portray with notifications, to control the user's attention - a vocabulary of auditory artefacts which we may use to command attention. The aim of this experiment was to find apply this to the cross-device use case, and to consider the methods for getting a user's attention to transfer to the companion content in the most efficient and preferable way. Based on the previously discussed 
methods and the information known about how we process audio/visual stimuli, we designed methods to shift users' attention across devices and tested them in a typical scenario.

\subsubsection{Design of Stimuli}

As described in Table 8, there were six methods tested: one (baseline) where the content just appears on the tablet (no. 1), one animated visual method on the tablet, to explore the effect of motion on the visual notification (no. 2), two auditory techniques on the tablet - an earcon, to explore the effect of an abstract sound clashing with the sound scape of the experience (no. 3), and an auditory icon to explore a sound that is less disjunct (no. 4). Also, there were two methods that notified the participants on the TV itself by providing an icon (Figure 18) - one stationary (no. 5), and one animated (no. 6), we did this, again, to explore the effect of motion, but also to consider the effect of placing materials on the more motion rich television, compared to notifications on the tablet.

\begin{tabular}{cll} 
No. & Type & Description \\
\hline 1 & Content appears & Content simply appears on tablet \\
2 & Content shakes & Content shakes on tablet \\
3 & Earcon & User notified with musical sound \\
4 & Auditory icon & User notified with related sound \\
5 & On TV & Icon appears on bottom right of TV \\
6 & On TV \& shaking & Icon shakes in bottom right of TV \\
\hline
\end{tabular}

Table 8: Methods used to attract the attention from the TV.

We designed the shaking visual alerts on the device (no. 2) and on the TV (no. 6) to best gain attention and negate change blindness (a user not noticing a visual stim- 
ulus when introduced due to distraction elsewhere) [129]. As peripheral vision is poor, in terms of acuity, but tuned to detect motion (specifically horizontal) [43] we designed them to shake diagonally to attract a user's gaze. To avoid the content going unnoticed and combat the auditory equivalent of change blindness we designed two types of auditory notifications - one totally disjunct from the sounds in the show (no 3); and a more related type (no. 4). The earcon (no. 3) featured a musical sound, which was designed to be disjunct from the sounds associated with the show (natural sounds, incidental music, presenters speaking); and, the auditory icon (no. 4) featured related sounds - for example, the sound of a river running when a fish is in shot on the TV.

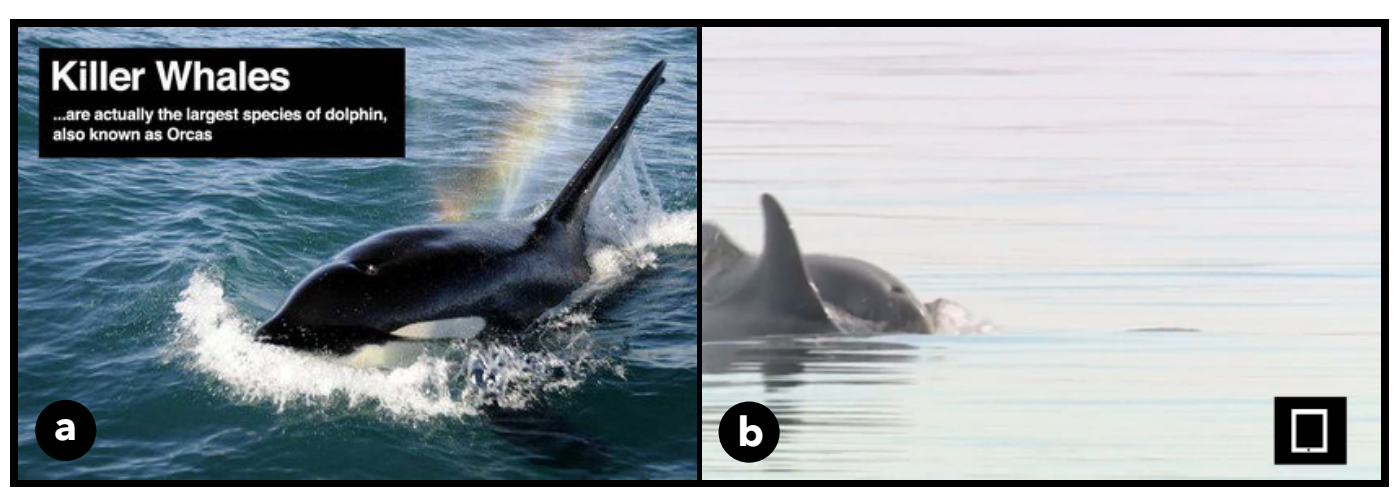

Figure 18: Example content used in the mediating attention study: a) shows a piece of tablet content used in the study, and b); its corresponding television timeframe. Note the visual notification in the bottom right hand corner, indicating that a new piece of tablet content is available.

We recruited 18 participants from general staff mailing lists at the BBC. Their ages ranged from 25 to 48 , with a mean of $36(\mathrm{SD}=7.24)$, with 11 identifying as male and 7 female. Users reported an average of 2.53 hours of TV viewing per day (SD 
$=1.43), 16(89 \%)$ of the participants stated that they engaged with mobile devices while watching TV, and $10(56 \%)$ stated that they are easily distracted by other electronic devices while watching TV.

We conducted the study in a usability lab, rigged as a generic living room. The participants were in a typical scenario for such a viewing experience; that is, sitting on a sofa in front of a 42 inch modern TV with a tablet computer (iPad) on their lap. As depicted in Figure 19, participants were filmed from directly in front by a camera on top of the TV, and also from above the $\mathrm{iPad}$, allowing for us to make recordings of the participants and use them for later analysis. The participants were first talked through the study and then asked to fill in the consent and demographics forms. They then watched the television show, which was an excerpt from Autumnwatch (similar to content used by Brown et al. [23]). During this, second screen content was introduced at two to four minute intervals to complement the programme. This content was simple images and textual information (as in Figure 18). Each time a piece of content appeared it was accompanied by an attention mediating method (for example the content appeared on the tablet and visually shook side to side). Upon completion, the user was asked to fill in a post-study questionnaire that allowed us to gauge their impressions (full questionnaire included as Appendix D).

In terms of experimental design, we used a Latin square arrangement to ensure that the participants were exposed to the methods in a different order each time so that we could observe their impact irrespective of the content on the TV or the tablet. Reaction times were our primary objective metric. To observe these, we inspected the time between the content appearing on the tablet and each participant's attendance to it. By analysing the video (Figure 19) we could track the 


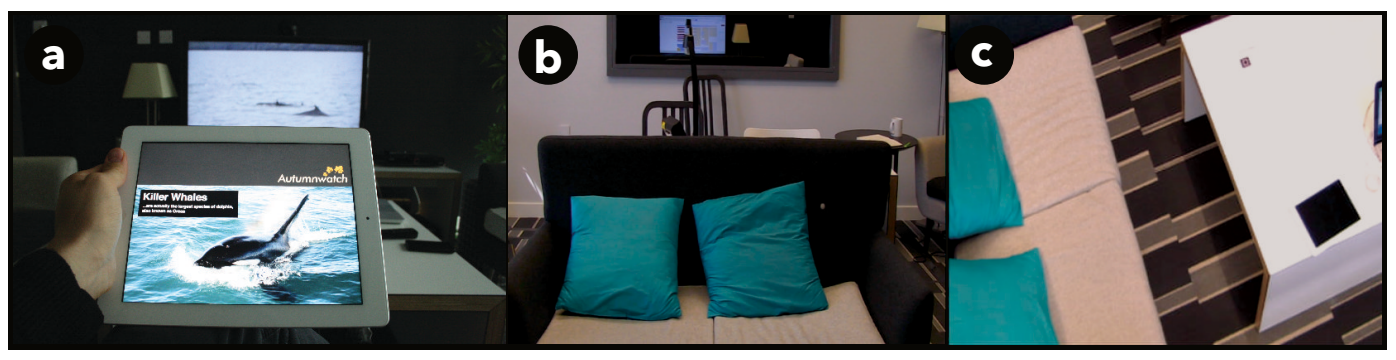

Figure 19: Participant perspectives of the mediating attention study. a) shows the setup from the perspective of the participant, b) shows the camera used to track the gaze of the participant (fixed on top of the television); and c) shows the camera placed above the participant to capture interaction with the tablet.

participants' eyes and easily infer where they were looking and at exactly what time. To ensure consistency we adhered to a strict set of criteria to classify the participants' gaze, and verified our results with a second sampled analysis, and with an external party. We then framed our objective findings by using a post-study questionnaire to gauge how much the methods got their attention and to assess what they preferred.

\section{$7 \cdot 4 \quad$ RESULTS}

In this section, we describe the objective findings (reaction times). We then discuss the participants' subjective impressions of the stimuli by analysing their ratings, and briefly discuss their comments.

\subsubsection{Objective Results}

Figure 20 shows that peripheral methods (on-device auditory and visual stimuli) resulted in the fastest overall reaction times from the participants, and that notifying the participants on the television resulted in the slowest. 


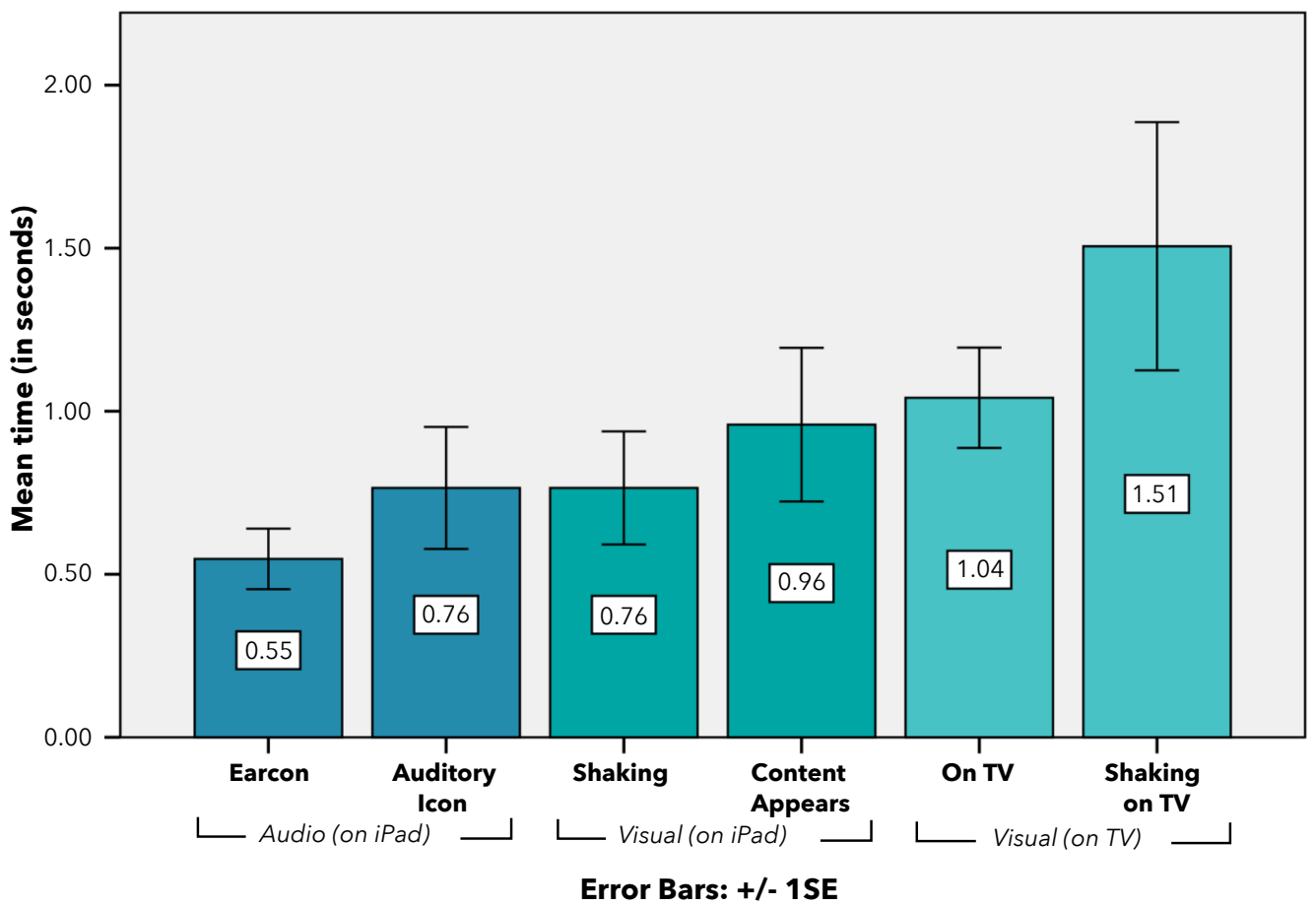

Figure 20: Participants' reaction times in ascending order. From left to right we see that the auditory methods yeild the quickest reactions, followed by the visual on-tablet stimuli, finally followed by the on-TV methods.

From Figure 21 we see a positively skewed distribution as most of the reaction times were near zero, with a taper off towards the higher times. To normalise the data for statistical analysis we conducted $\log 10$ normalisation, which Tabachnick and Fidell advise for this type of distribution [132] (Section 4.1.6).

We conducted ANOVA to examine the data and found significant overall variance $(\mathrm{F}(5,13)=4.051, \mathrm{p}=0.019)$. Moreover, upon conducting post-hoc (Bonferronicorrected) tests on the stimuli conditions, we found significant differences between the Earcon and the stimuli appearing on the television, both static $(\mathrm{p}=0.002)$, and 


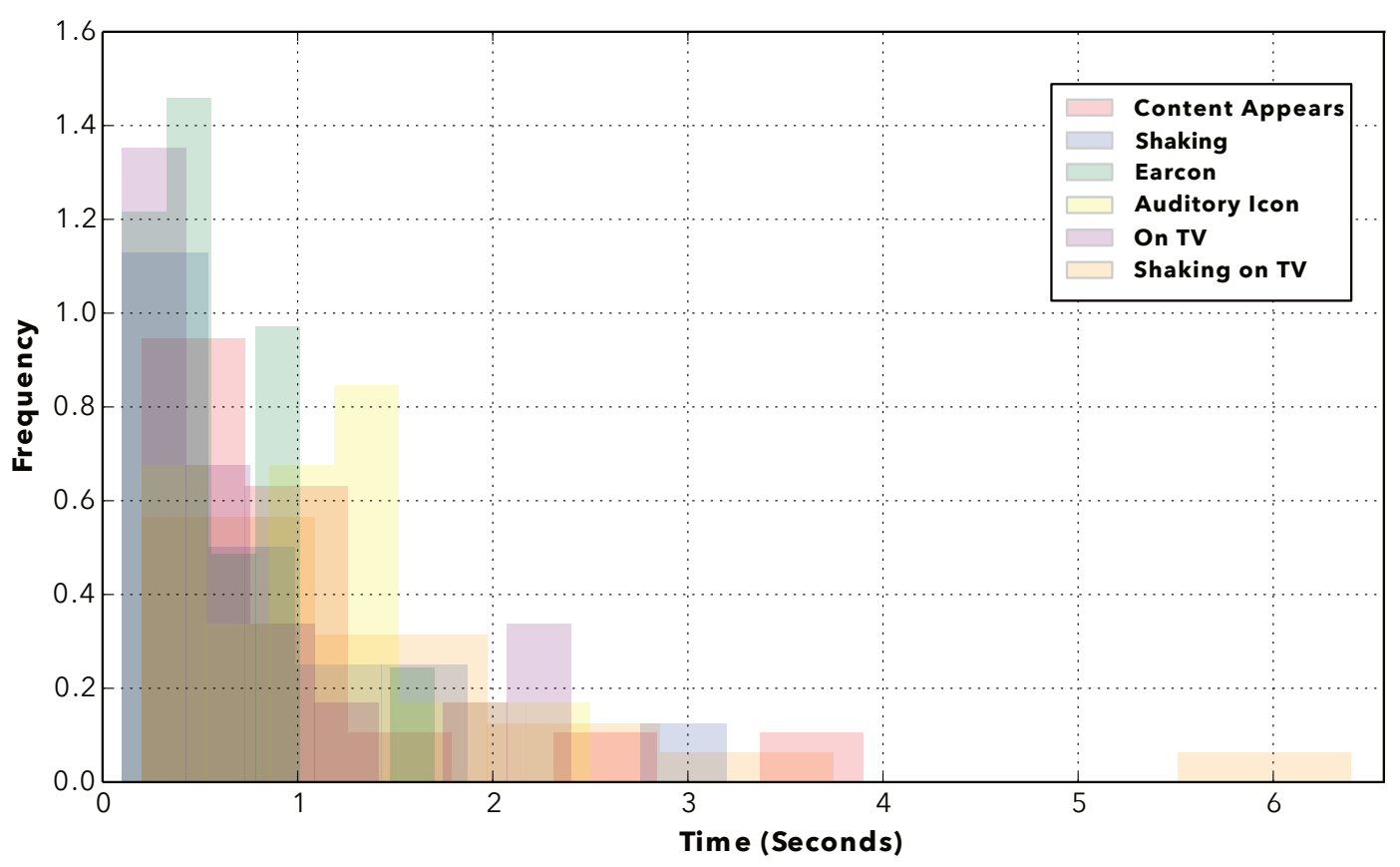

Figure 21: Histogram of response times: generally participants responded quickly to the stimuli, resulting in a positively skewed distribution around zero.

shaking $(\mathrm{p}=0.002)$, and also compared to the content appearing on the device $(\mathrm{p}=$ o.029). Auditory icons also yielded faster attention shifts than when notifying the user on the television, when static $(\mathrm{p}=0.040)$ and shaking $(\mathrm{p}=0.038)$

Notably, a shaking alert on the iPad appears to attract attention to the tablet quicker than the non-animated cue, but the opposite occurs on the TV.

\subsubsection{Subjective Results}

Participants were asked to describe how well they believed different cues had performed in attracting their attention to the tablet. As shown in Table 9, 11 participants $(62 \%)$ strongly agreed that the earcon was most effective at attracting their 
attention, followed by the auditory icon. Both were preferred to the content simply appearing without additional stimuli, significantly so for the earcon $(Z=2.59, \mathrm{p}=$ $0.005)$.

In terms of subjective preferences, auditory icons (the sounds that related directly to the show) were most favoured, significantly more so than the content simply appearing $(Z=2.03, p=0.021)$. Also, approval of both TV-based alerts was significantly higher than the no alert case $(Z=2.16, p=0.016$ and $Z=1.86, p=0.032$ for non-shaking and shaking respectively). Several participants noted that they used the notification on the TV to delay when they viewed second screen content, for example, "I think the TV icon was best, I can look at the and device in my own time then".

\begin{tabular}{llllllll}
\hline & $\begin{array}{c}\text { Missed } \\
\text { Stimuli }\end{array}$ & $\begin{array}{l}\text { Strongly } \\
\text { Disagree }\end{array}$ & Disagree & Neutral & Agree & $\begin{array}{c}\text { Strongly } \\
\text { Agree }\end{array}$ \\
\hline Content Appears & 11.1 & 0.0 & 11.1 & 5.6 & 38.9 & 33.3 \\
Content Shakes & 33.3 & 5.6 & 0.0 & 0.0 & 22.2 & 38.9 \\
Earcon & 22.2 & 0.0 & 0.0 & 0.0 & 16.7 & 61.1 \\
Auditory Icon & 16.7 & 5.6 & 0.0 & 0.0 & 27.8 & 50.0 \\
On TV & 16.7 & 0.0 & 16.7 & 11.1 & 16.7 & 38.8 \\
Shaking on TV & 22.2 & 0.0 & 5.6 & 11.1 & 16.7 & 50.0 \\
\hline
\end{tabular}

Table 9: Participants' reported attention gained for each method, expressed as percentages. Darker colours indicate higher frequencies.

The uncued (content appears) case scored very poorly, and comments from the participants imply that they believed that they would miss the content if not alerted to it; "[I] was constantly checking to see if I'd missed it." - "It felt a little surprising just to discover content”. On the other hand, the highest scoring method, the auditory icon, was mostly praised because of its ability to link the show to the second screen 
content, for example, "It felt [a] more sympathetic and complimentary way of identifying that more content was available." and "it felt less intrusive". Conversely, it was apparent that some participants found audio distracting in general and would have preferred more discreet methods such as vibration. Notably, we found a correlation between how much a user believed something got their attention, and how much they liked it (Spearman's $\rho=0.384, \mathrm{p}<0.001$ ).

\begin{tabular}{lllllll}
\hline & $\begin{array}{c}\text { Missed } \\
\text { Stimuli }\end{array}$ & $\begin{array}{l}\text { Strongly } \\
\text { Disagree }\end{array}$ & Disagree & Neutral & Agree & $\begin{array}{c}\text { Strongly } \\
\text { Agree }\end{array}$ \\
\hline Content Appears & 11.1 & 5.6 & 33.3 & 16.7 & 22.2 & 11.1 \\
Content Shakes & 16.7 & 0.0 & 0.0 & 9.0 & 27.8 & 5.6 \\
Earcon & 5.6 & 0.0 & 16.7 & 16.7 & 7 & 11.1 \\
Auditory Icon & 11.1 & 0.0 & 5.6 & 27.8 & 33.3 & 22.2 \\
On TV & 22.2 & 0.0 & 5.6 & 22.2 & 38.9 & 11.1 \\
Shaking on TV & 38.9 & 0.0 & 5.6 & 22.2 & 11.1 & 22.2 \\
\hline
\end{tabular}

Table 10: Participants' reported general preference for the stimuli, expressed as percentages. Darker shades indicate higher frequencies.

Table 10 shows that over a third (39\%) did not notice the notification shake on the TV. Some comments indicated that they did not notice such subtleties because their attention had already been diverted to the tablet; "because it happened at the same time [as the alert on the tablet] I felt unprepared. The [secondary] device caught my attention".

Reaction time data and the subjective feedback indicate that auditory methods performed best at quickly attracting users' attention to companion content. Likely causes of this observation are: a) we process auditory information significantly 
faster than visual [126], b) that peripheral stimuli (in general) cause a strong disjunct from the TV show, and c) that, in the case of the earcon, its lack of ecological coherence with the TV content forms a more explicit highlighting of change in the content on the tablet. Note, however, that reaction time may not be the most important measure: the subjective data shows that not only do participants' opinions vary greatly, but also that the fastest method (the earcon) for getting attention onto the tablet is not generally as favoured as the auditory icon.

We believe there are two reasonable explanations for the notifications on the TV being the slowest: Firstly, several participants reported that they used it as a method of delaying a voluntary switch of attention. Secondly, main screen alerts attracted (and split) participants' attention first delaying a shift of focus to the second screen. Finally, we believe that the shaking effect on the TV did not aid the participants in noticing the content on the iPad quicker was because its motion focused their gaze onto the TV and away from the tablet in their periphery.

This chapter has investigated methods for attracting attention to companion content when a user is engaged with the related TV programme. We found that:

- Users react quicker to the availability of refreshed second screen content when alerted by means of peripheral stimuli, especially through the modality of sound;

- many participants responded positively to alerts on the TV itself, as it supported consciously delaying shifting their attention to the companion content until a moment of their choosing; and 
- most vitally, participants much prefer their attention to be mediated, to ensure they did not miss parts of the primary material (constantly glancing at the tablet to check for new content), or second screen content (missing it because of their focus on the TV).

Our findings suggest that of dual screen user experiences should consider how closely in time the companion content needs be synchronised to the main screen content and cue the user appropriately. If, for example, the content is relevant to only a few seconds of the show, one may wish to use attention mediating techniques which have been found to cause more involuntary, faster attendance to the second screen, such as peripheral/auditory methods. However, if the relevance of the material to the notification cues spans minutes rather than seconds, methods that use notifications on the main TV screen will likely yield better results. The results of the investigation presented in this chapter suggest that when developing cross-device applications, where user attention is divided across devices with ambiguous states, that attention design with additional stimuli is strongly advised.

In terms of the research questions of this thesis, we see clear evidence here supporting RQ3; that we can intervene into a user's attention and improve its distribution by providing an additional cue of where to look and when. This is reflected in the strong preference for mediating attention over the default (no attention mediation). Further, regarding $\mathrm{RQ}_{4}$, despite the fact that we are explicitly intervening in attention, of the five methods we investigated, we see a variety of user behaviours around the way users are in control of the way they manage their attention. Earcons, for example afforded the user very low autonomy as they were drawn quickly to the content. Whereas, notifications on the TV allowed users to defer 
when they looked at the content; to some degree empowering them to look in their own time.

In the previous two chapters we have considered methods by which we may alleviate the attentional disjuncts in the cross device experience through adapting the complexity of the content, with a focus on understanding the benefits/costs of user autonomy. In this chapter, we pivoted to show that it is also effective shift control to the content creator, and guide their experience with supplemental audio/visual notifications. By extending the current work in the area and conducting an empirical study of additional stimuli, we provided a series of guidelines for this use case in terms of speed of attendance (urgency), and offer a palette of tools for designers to consider when creating cross-device experiences. 


\section{CHAPTER EIGHT}

\section{Display Commonalities}

So far in this thesis we have explored how we may design cross-device experiences by further understanding the use case, by varying the complexity across devices, and through invoking shifts in attention at opportune moments. In chapters 5 and 6 our focus was to provide interventions that adapted the material on the tablet to better fit preferred complexity levels of users - to some extent controlling user's attention. In Chapter 7 we took this further by initiating cross-device shifts of attention through notifications; manipulating attention and shifting autonomy from the user.

In this third and final intervention we explore techniques that aid users in managing their own cross-device attention with more autonomy. By utilising an increasingly popular living room trend - screen mirroring - we aim to promote heightened awareness of unattended devices, and therefore optimise distribution of attention.

Towards answering the research questions RQ3 and RQ4 outlined in Chapter 3 we aim to find if mirroring unattended devices' content is an effective method of attention management. In addition, we wish to explore the extent to which allowing the user to have more control of their attention (i.e., not invoking shifts as in the previous chapter) yields any benefits for each context explored.

We explore a concept which we term as display commonalities - the mirroring of an unattended display within another so that we may monitor it. First, we consider 
the previous work on screen mirroring broadly, then focused around cross-device media experiences. Then, we progress to discuss our design rationale and reduce the design space for testing with practitioners. Finally, we conduct a large-scale empirical study in two scenarios with 40 participants to further evaluate and understand this avenue of exploration.

\subsection{SCREEN MIRRORING IN THE CONNECTED HOME}

The focus of this chapter is the use of duplicate elements across dual-screen UIs to improve UX. Much of the work in the second screen scenario explores how we may measure $[23,66]$ and, as previously demonstrated in this thesis, intervene in users' attention. However, this does not consider the full capabilities of the connected living room for the second screen use case. The research around dual-screen mirroring, though fruitful for shared experiences in the living room, generally focuses on how we may design to enhance shared viewing experiences (c.f. the work of McGill et al. [96]).

The sales of internet-enabled 'Smart' TVs is increasing dramatically - information services company, IHS Markit, suggest that over $50 \%$ of households in Japan, the US and Europe will have smart TVs by 2019 [104]. Due to our televisions' increased connectivity to our home networks and the internet, it is now possible for many of us to connect our mobile devices to our televisions to control and share content the mobile device, as discussed in the framing material of this thesis, is beginning to replace the remote. The direct mirroring of a device's screen, or indeed extensions of the screen (see 22 for examples) is quickly becoming an everyday part of the modern living room landscape. 


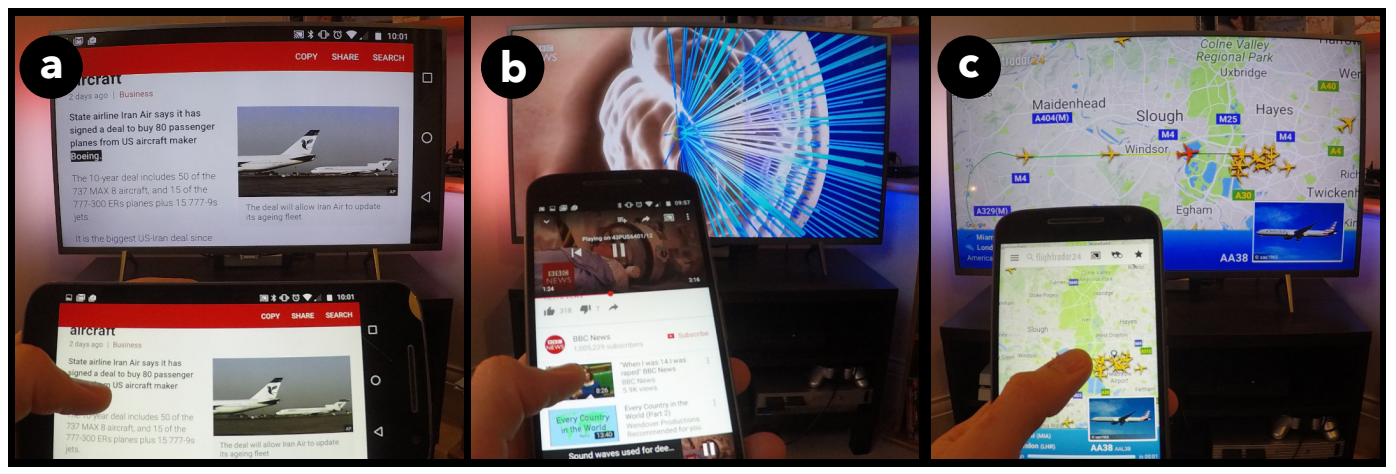

Figure 22: Here we show some examples of screen casting from a mobile device to a TV in the modern livingroom via WiFi. In a) we see a user mirroring their whole screen to the television, for example to share a new story. In b) we see a user queueing up videos from their mobile to watch - utilising the rich touch affordances of the mobile, in tandem with the superior viewing angle of the television. And, finally, we see the user casting their display to act as a shared, ambient display of real-time flight traffic over Heathrow Airport.

Statistics from a 2013 study with 2600 users suggests that screen mirroring awareness was at around 40\% [56]. As of the time of this thesis submission (2017), little recent data exists on screen mirroring penetration, however, given the increasing proliferation of casting services such as Google's Chromecast ${ }^{1}$ and Apple's AirPlay $^{2}$, it is likely to be an increasing trajectory. Such interactions afforded by casting services allow us to utilise the superior interaction capabilities of our touchscreen handheld devices, in tandem with the superior visual capabilities of a large display, such as a television.

Empirical investigations have looked at how to best cast a device's whole screen to a TV. For example Fleury et al. [47] investigated user preferences for screen mirroring with a mobile and a TV. Screen mirroring has been further explored with

1 Google Chromecast: https://goo.gl/xgbwOb

2 AirPlay: https://goo.gl/4c03MR 
an aim to promote mutual sharing of content between users from their personal mobile devices. For instance, McGill et al. [96] look at how, in a shared viewing experience, we may use the affordances of screen mirroring to foster enhanced collaboration between users of a TV, and how we may design to engrain equal participation in mirroring. McGill's work approached the problem of the digital bubbles we inhabit when we interact with our mobile devices towards overcoming it by mirroring devices onto a larger, shared display.

In terms of large-scale adoption, many online streaming services now offer interactions on a second screen to support their viewing, for example Amazon Prime Video allows for the casting of video to another display (typically a TV) while engaging with second screen content: the $X$-Ray service [2] provides encyclopaedic, time-synchronised, content and information about people featured in a film when a user interacts.

When considering the design space for integrating video feeds into a second screen we sought inspiration from the early interactive TV literature, which often focused on the design of EPGs (Electronic Programme Guides). Although, historically, remote controlled EPGs often consisted of a video embedded into the top right hand corner of the TV (screen-in-screen) - so that a user may attend to a programme and channel surf - handheld EPGs (c.f. [33, 131]) have not incorporated this. More recently, however, browser-based video experiences have used similar design ideas (e.g. the Floating Youtube Chrome extension ${ }^{3}$ ) - as the user scrolls down the page, to read an article for example - the video embeds into the corner and follows 
as they scroll. Further, to allow for multitasking, recent, larger iPad devices also implement a screen-in screen feature to allow users to attend to video ${ }^{4}$ while engaging in other activities. Such designs, however, only allow for the direct mirroring of the mobile device's screen, and do not afford users the opportunity to integrate individual elements of the TV display into a mobile user interface, and vice versa.

In considering alternative approaches for embedding a video stream into the periphery of a handheld display, there is a clear tradeoff between video detail and screen space: as the video feed becomes bigger more of the content that is native to the device is occluded. Knoche et al. [82] investigated users' optimum viewing ergonomics for video on mobile devices and found that, given the relative smaller distance between the user and the device (compared to the user and the TV), many mobile devices afford an acceptable viewing angle. However, shrinking this video to show other content on the mobile device is likely to impact the ideal video angle, an effect which is likely to get worse for smaller devices. The optimum design would allow sufficient screen real estate for the content mirrored from the 'other' device, without obfuscating the material on the display the user wishes to attend.

In addition to the loss of video detail in such screen-in-screen designs, such approaches generally cause obstruction to viewing content in the corner of the screen. To alleviate this issue, we considered the option of non-opaque displays. Kamba et al. [75] considered, in their early work on mobiles, how the transparency control widgets in a display can act as a method to extend smaller screens - to allow for UI elements to be visible. Such methods, in our case, would allow for the whole 
screen to be occupied with the companion content while still allowing a user maximised resolution of the video. However, this use of transparent overlay is bound to introduce additional issues in terms of visual ergonomics. 


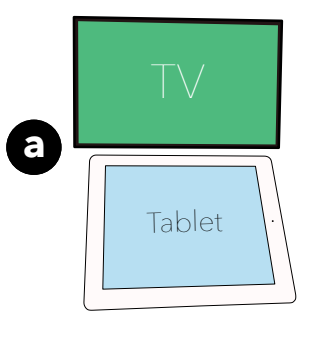

b

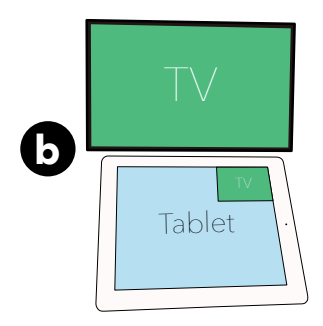

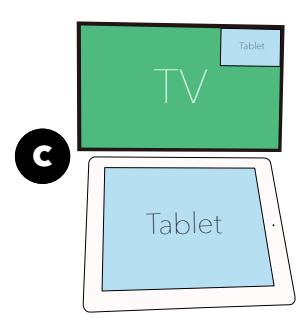

d

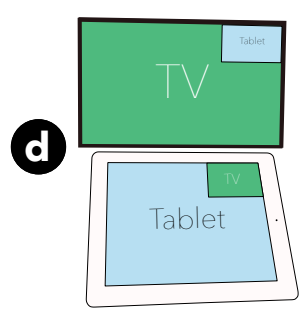

(h)

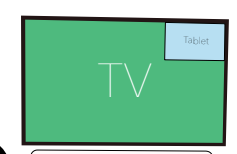

(9)

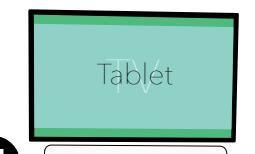

f

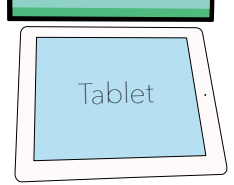

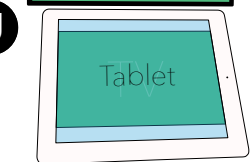

(1)

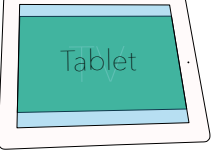

(i)

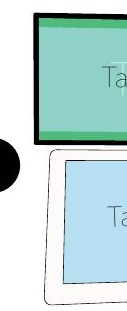

Figure 23: Initial design space discussed with practitioners: we considered the permuta mentioned techniques to stimulate discussion in our focus group. a) depicts the 'default case, b) the TV material being mirrored from the TV to the corner of the tablet, c) the tal to the TV, and d) a combination of b) and c). In e) we depict mirroring the TV behind tablet, with the opacity of the content lowered to allow for the user to see through it, a reverses this concept and projects from the tablet to the TV. Technique g) is a combinati h) considers a hybrid of projecting from the tablet to the corner of the TV while simulta TV content behind the tablet screen. Finally, i) shows the inverse of $h$ ) - placing the tab] programme on the TV, and the TV programme in the corner of the mobile device. 
Consolidating the literature discussed in the previous section allowed us to arrive at the design space depicted in Figure 23. To progress our investigation, we were inspired by work which uses experts to whittle down a large set of designs, before exposing them to users (c.f. Marsden et al. [92]). In particular, we considered work such as that by Geerts et al. [53], where the opinions of experts are garnered towards designing better second screen applications. To further consider methods which would be viewed feasible in terms of an editorial perspective we engaged with content designers and creators at a major broadcasting corporation through a focus group. The benefits of doing so are practical and ecological; as it allowed the expert consideration of the fully-populated design space with a manageable set of options for audience user research determined by considered, reproducible practice.

We recruited four professional designers at the broadcaster, along with a creative director. Designer participants are referred to as D1 through D5. The participants' professional practice included a focus on designing the 'live experience', to accompanying produced content - for example, real-time sports statistics and social media integration. Their core skill-sets and backgrounds were broad - ranging from graphic design to programming.

\subsubsection{Participants}

Designer participants, outlined in 11 , are referred to as $\mathrm{D}_{1}$ through $\mathrm{D}_{5}$. The participants' professional practice included a focus on designing the 'live experience', to accompanying produced content - for example, real-time sports statistics and 
social media integration. Their core skill-sets and backgrounds were broad - ranging from graphic design to programming.

\begin{tabular}{lllll}
\hline Participant & Gender & Age & Role & Companion \\
\hline P1 $_{1}$ & Male & 35 & UX Designer & No \\
P2 $_{2}$ & Male & 42 & Creative Director & Yes \\
P $_{3}$ & Male & 33 & UX Designer & Yes \\
P $_{4}$ & Male & 22 & UX Designer & Yes \\
P $_{5}$ & Male & 32 & UX Designer & No \\
\hline
\end{tabular}

Table 11: Designer participant demographics - we recruited designers with experience designing for web experiences. Three of our participants noted having experience creating experiences for dual-screen - for example, providing live second screen sports statistics.

\subsubsection{Procedure}

The study was intended to allow the design space to be refined by the expers, in order to identify which were the most viable design approaches warranting further exploration. We built nine working prototype dual-screen applications as example pieces of content for the companion application, each manifested with the proposed commonality methods described in the design space (see Figure 23), contrasting these to a baseline with no commonalities.

We first explained the eight display commonality methods without any suggestion of what they may be used for. Then we handed around the prototypes on the tablet, while casting the video to a TV. Participants freely discussed the prototypes then, at the end of interacting with each one, we asked them to reflect on their experience - noting the positives and/or negatives, along with some potential envisioned usage. Finally, after interacting with all UI we asked them to state their preference 
for all UI in terms of efficacy and ease of use. We made audio recordings of the participants' comments, transcribed the data, and conducted an analysis.

\subsubsection{Results}

In general, practitioners strongly favoured UIs that mirrored the TV on the tablet - three preferred UI b) and two preferred UI e). On-tablet mirroring of TV was positively received by the practitioners. A recurring theme in our discussions was that any overlay on the TV puts the designer at risk of encroaching on the TV programme - occluding important elements. The designers referred to the TV as 'sacred space' - "I just feel like that space there [the TV]. That's the primary - that's sacred. And I don't feel like you should really encroach on that" (D1). They generally favoured methods that either did not cast to the TV, or methods that did so discreetly (for example h) and c)). They noted that persistent material would negatively encroach on TV's territory, and that it should only be placed at very specific points as a call to action - "kind of at trigger points letting you know that there is something on your companion screen, so that you can be involved with it."(D2).

Designers responded positively to on-tablet mirroring of video content - both in the corner of the handheld screen - b), and presented behind the handheld device's native content, e). Screen-in-screen on tablet, b), was the most preferred because it was seen to work for basically all types of envisioned usage (e.g. companion content and browsing of the web). Some preferred it to the overlaid content - e), because it allowed them, as designers and viewers, to compartmentalise the mirrored TV and the supporting content. However, the positive comments around mirroring from the TV to the mobile device were also mixed with concerns about users focusing wholly on their mobile device - negating the reason to have the TV in 
the background (despite its superior viewing quality) - "I kind of feel like you don't have to look at the TV. But I like how you can look at the view and then back at the TV so you don't have to deal with 'both"'(D2).

The opacity overlay was a divisive UI when used on the tablet computer - some $(2 / 5)$ preferring it to the screen-in-screen. The positives for this UI mostly edged around the fact that it was aesthetically pleasing (that it worked like a wallpaper), that it afforded a larger screen, and that it allowed the users to absorb both streams of information simultaneously - "I do actually prefer it when it's the whole video behind the screen, rather than when it's in the corner. Like I said before - you're watching the TV or reading the text. Whereas with this it feels like you're absorbing both bits of information" ( $\left.\mathrm{D}_{3}\right)$. Criticisms focused around the fact that opacity-overlay UIs may not work for non-designed experiences such as free browsing - the opacity of the overlay may occlude some parts of the video display. Overlay on tablet (e.g., e)) was generally seen to afford 'designed experiences' more - “...information overlaid on top of video - it looks somehow compelling and seamless. But you know, if it was anything other than these pleasant meditative screens it would be a lot more competing” (R2).

Dual-mirroring (mirrored elements on both UI) was generally seen as excessive and thought to introduce redundancies. The designers tended to prefer more universal UI - interfaces which could be applied to the most scenarios, over powerful ones which could be used in few. UIs which were thought to afford only one type of viewing - for example the overlay on the TV - which they only saw useful for brief sharing, were generally not praised. And all cases where content was mirrored onto the TV with an opaque overlay were generally noted to be good for only one thing - sharing content with others. The favoured method of mirroring 
to the TV was to keep the mirrored content discreet by placing it in the corner of the display c). Finally, weighing up the benefits of each, they reached a consensus that the most viable methods for future exploration were: b) - the video feed from the television in the corner of the handheld device; c) - the mirroring of the mobile device to the corner of the TV; and e) the TV video feed behind the material on the mobile device.

\subsubsection{Reflections on Focus Group}

The focus group allowed us to refine our design space, informed by the insight of those who would be tasked with integrating such concepts into their systems. Our focus group allowed us to make the following conclusions:

- Mirroring back from the TV (e.g. - b), d), e), g), h) and i)) to the device was considered viable as a commonality method;

- Screen-in-screen mirroring on the tablet and TV - b) and c) respectively could be applied to most scenarios they envisioned;

- The TV is sacred - the use of mirroring fully over TV content (e.g. f), g), and i)) is generally advised against for most scenarios;

- Dual-mirroring (e.g. d), g), h) and i)) was generally viewed as cluttered and redundant.

8.4 EMPIRICAL INVESTIGATION OF COMMONALITIES

The aim of the focus group was to whittle the design space down to three preferred techniques for further validation with users. By running this study with 
end-users - those who engage with second screen material in their everyday viewing - we wished to capture preferences and gain further insight into the techniques. The three commonality conditions preferred by the practitioners were then used in a large-scale empirical user study. We investigated the subjective and objective effects of display commonalities from the perspective of end users (i.e. viewers). These three, and a no-commonalities baseline, are as follows (and depicted in 24):

- $\mathrm{C}_{1}$ - the baseline condition with no commonalities. This is the typical experience of a second screener as it stands;

- $\mathrm{C}_{2}$ - mirroring from the TV into the right hand corner of the tablet computer;

- $\mathrm{C}_{3}$ - mirroring from the TV behind the content on the tablet computer screen with the opacity of the overlaid content's alpha set to 0.7 ;

- $\mathrm{C}_{4}-$ mirroring from the tablet to the top right hand corner of the TV.

\subsubsection{Participants and Study Environment}

We recruited 40 second screeners $\left(\mathrm{P}_{1}-\mathrm{P}_{4} \mathrm{o}\right)$ from Swansea University - both students and staff. Participation was rewarded with $\mathfrak{f}_{5}$. The average age of our participants was 32.5 years old $(\mathrm{SD}=8.6)$ - 26 identified as female, and 14 male. On average our participants watched $2.34(\mathrm{SD}=1.65)$ hours of TV per day, and either strongly agreed (31) or agreed (9) that they regularly engage with touch screen devices regularly, and all were second screeners to some degree. Five had noted using a companion application to support a TV programme before. All studies were conducted in a research lab configured as a living room (pictured in Figure 25). We used a 32 inch HD TV, which was connected to a laptop. We cast video from the 

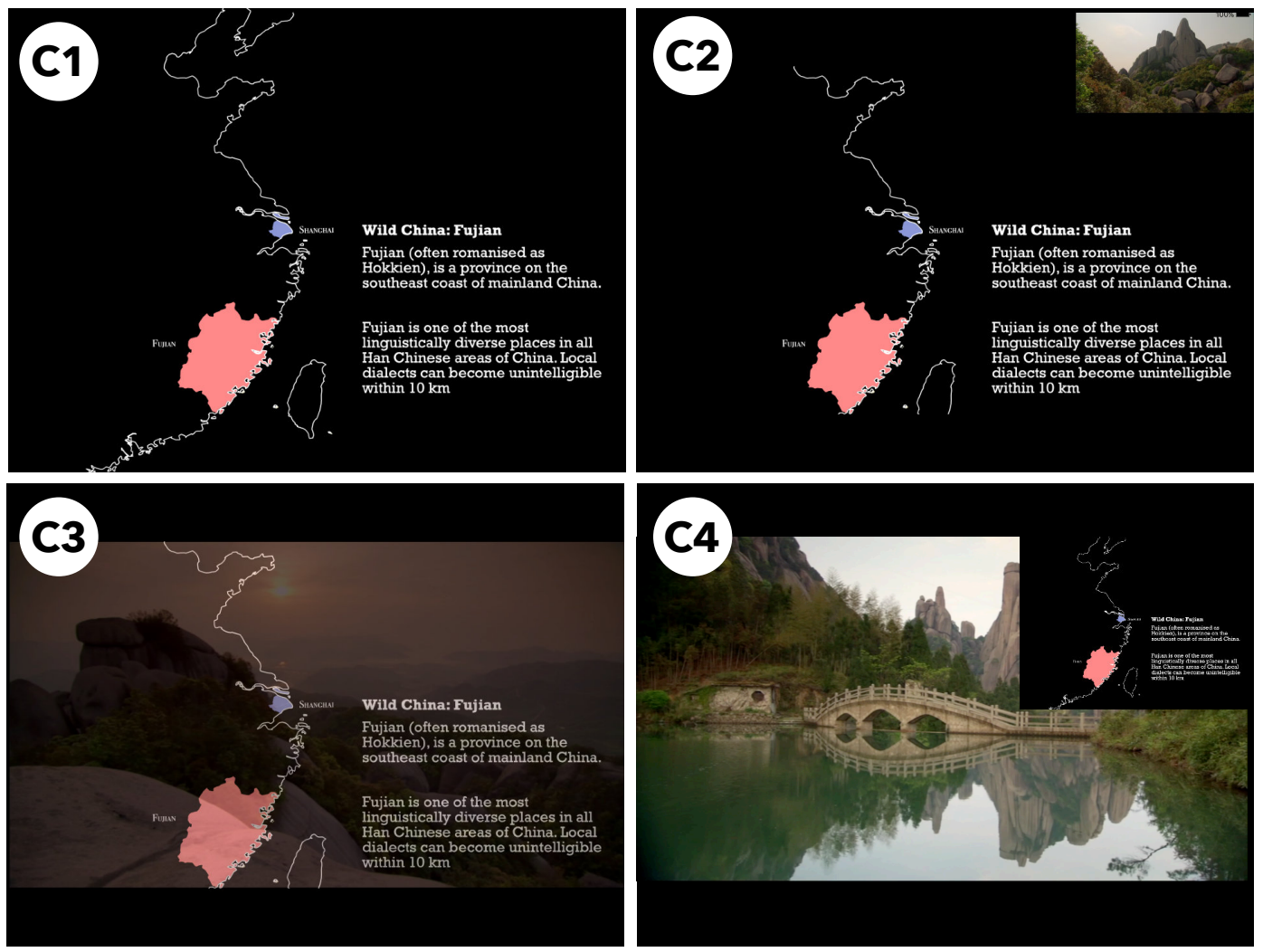

Figure 24: Each condition, shown as one screen in the 'Wild China' application. $\mathrm{C}_{1}$ (tablet), $\mathrm{C}_{2}$ (tablet) and $\mathrm{C}_{3}$ (tablet) depicts their respective conditions running on the tablet for the Wild China applications. $\mathrm{C}_{4}$ shows the tablet content being projected to the television.

handheld device - an iPad - to this using AirServer ${ }^{5}$ over a personal Wi-Fi hotspot to minimise interruption, and ensure negligible latency between the video streams on each screen.

5 AirServer: https://goo.gl/R3UY76 


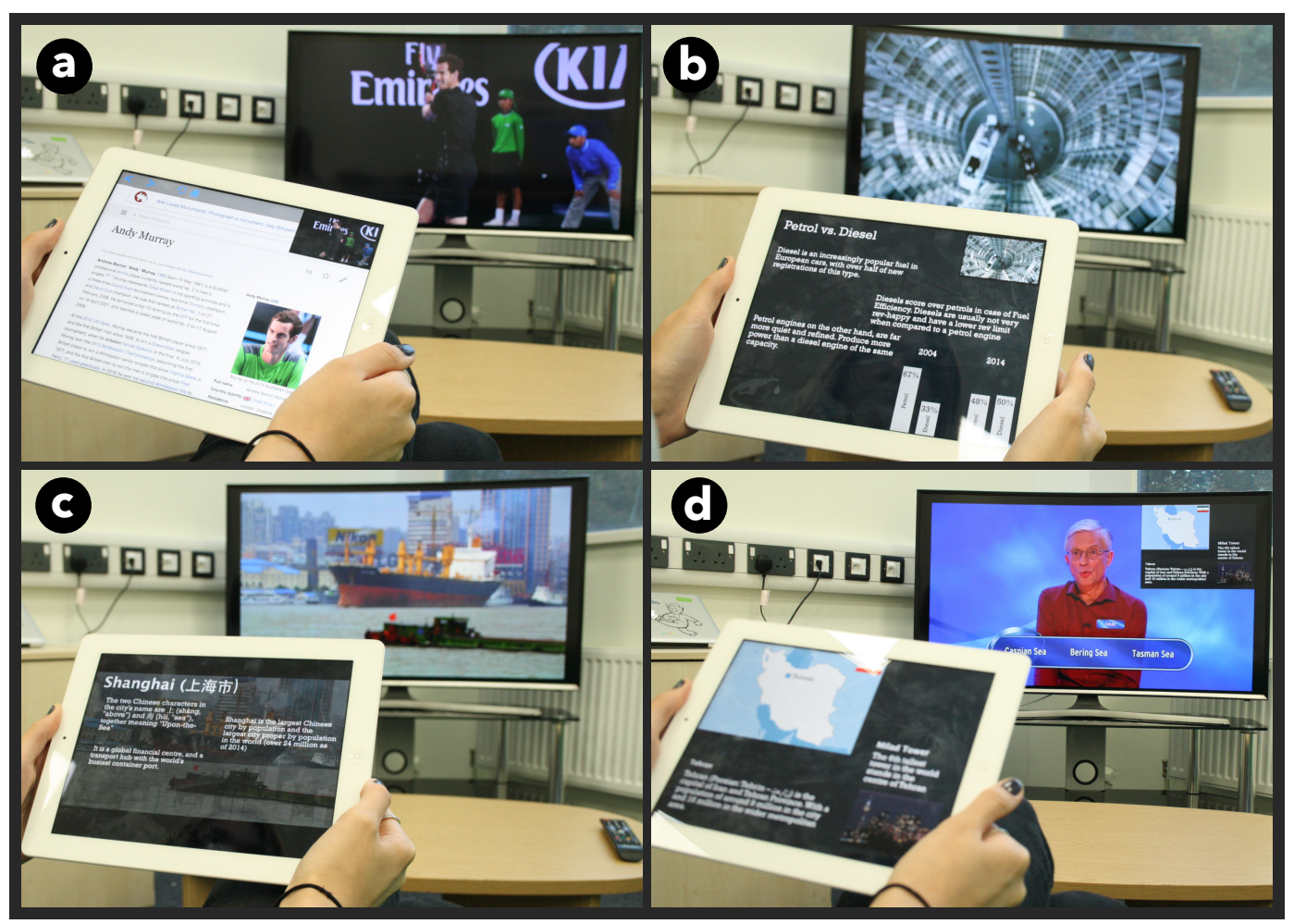

Figure 25: The four display commonalities: a user utilising display commonalities. a) shows the feed from the TV duplicated in the corner of the tablet as they browse related information on the web; b) shows the same concept is implemented for a companion application; c) shows the TV material is mirrored behind a companion application; and, d) shows the companion application appearing in the corner of the TV.

\subsubsection{Study Procedure}

Each study participant first read and completed a consent form, then filled out a demographics form to allow us to better understand our sample. The participants were then given their individual brief, dependent on the experimental group they were in (COMPANION APP or FREE-BROWSE - discussed in the next subsections). Following this they watched a diverse set of four clips (to mitigate genre effects, as 
discussed in previous chapters) from popular programmes (see Table 12), each followed by a questionnaire to evaluate their experience. Condition-wise ordering effects were mitigated against with a Latin Square design. Then after watching all four clips the participants filled out a post-study questionnaire to allow them to reflect on their whole experience, and an investigator then conducted a short semistructured interview. The interview questions focused around understanding if the participants noticed whether any of the conditions affect their attention management during the study, explicitly asking them to state a preference, and to explain their perception of each method.

\begin{tabular}{lll}
\hline Clip No. & TV Programme & Summary \\
\hline 1 & Wild China & Documentary about China \\
2 & Australian Open Final & Tennis game \\
3 & VW Scandal & Documentary about emissions scandal \\
4 & Eggheads & Quiz programme \\
\hline
\end{tabular}

Table 12: Table of clips to evaluate display commonalities - each programme was edited to run for approximately 5 minutes each.

The experiment followed one of two formats: the COMPANION condition - in which the TV viewing experience was accompanied by a dedicated companion app ( $\mathrm{P}_{1}-$ P2o), and the FREE-BROWSE condition, where the participants were free to browse the web $\left(\mathrm{P}_{21}-\mathrm{P}_{40}\right)$. The participants for this were assigned chronologically - after 20 participants, we began running the study with the FREE-BROWSE condition. We did this to explore the effects of each method so that our findings are generic to the two most common second screen cases, and to compare and contrast conditions. To ensure the validity of our between-participants results, we conducted statist- 
ical analysis of the participants' demographics using paired t-tests and found no significant differences between the populations.

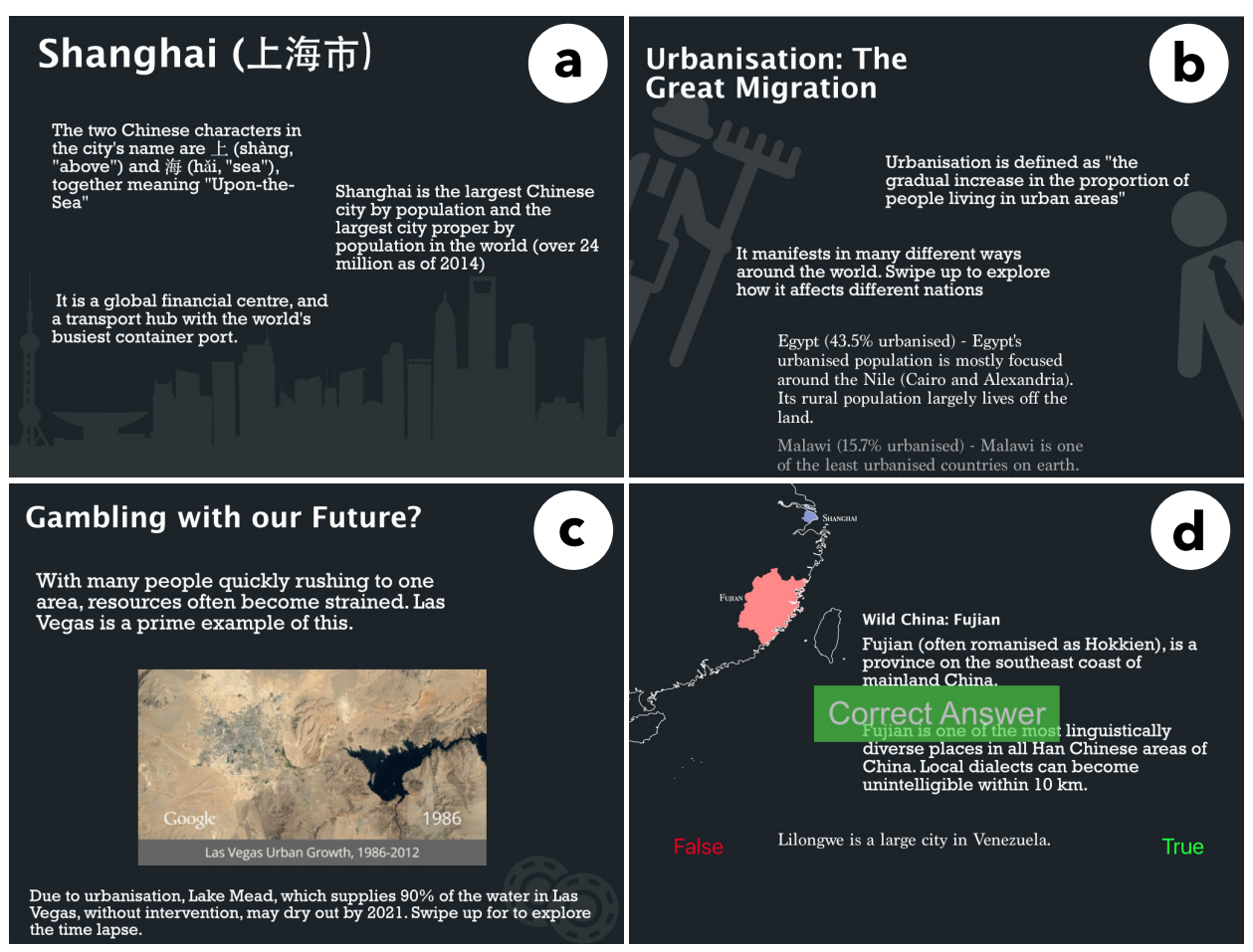

Figure 26: The Wild China Companion Application (depicted here with no commonality methods), in order of appearance to the user: a) shows a simple noninteractive plain text screen - these were generally a mixture of simple text and figures; $b$ ) shows the interactive text screen of the application - here participants could swipe up and explore more text; c), the animation screen - in this case the participants explored an interactive time-lapse of desertification; d) shows the quiz - here, as influenced by Chapter 5 , participants were given true/false questions related to programme and the tablet material to motivate engagement with the materials on both the television and the tablet. They were free to repeat the quiz as many times as possible, while also visiting other screens to fill in gaps in their knowledge. For each clip, the applications followed this same format but with programme-related information. 


\subsubsection{Companion Content Condition}

To explore the effect of commonalities on a designed experience we built four companion applications - one for each programme - which were representative of typical applications. Each app contained four main screens of programme-relevant material, which became available at key points in the experience, at which point the participant was notified of the new content with a notification sound and a visual cue. Figure 26 illustrates the companion application layout for one of the programmes the participants watched in the experiment. The four screens are indicative of the applications we used in the experiment.

\subsubsection{Free-browse Condition}

To explore the effects of the commonalities on the participants when actively engaged in knowledge query we created a simple web browser, with 'back','forward', 'home', and 'refresh' buttons for iOS with the relevant commonality methods included. We motivated the participants to engage with the TV material and the browser as they would in their regular browsing habits.

\subsubsection{Measures and Motivations}

Subjective metrics were gathered by asking participants several questions after each clip through the form of a questionnaire. We asked these questions with a motivation to understand the benefits of each commonality method in terms of how the participants take in information on the screens at the same time, how they perceive the cost of switching between them, the visual appeal of each technique, how aware of the 'other' device the participants were, and how each technique 
occluded the materials across the two screens (exact questions shown in Table 13). We also used a post-study questionnaire to allow the participants to compare and contrast the techniques, and reflect on the experience as a whole. In this, we asked them how they felt about each individual technique as a whole (exact questions shown in Table 14 and in Appendix E). Our hypotheses around the qualitative data were that the commonalities would allow the users to more positively manage their attention, and therefore we would expect to see this reflected in our Likert scores.

In terms of objective metrics, we measured the participants' interactions with the mobile device to determine how the conditions affected their experience. For this we logged time-stamped events on the device and stored them for later analysis. For the COMPANION condition we logged interactions within the app as a proxy for participant involvement with the second screen material - when the participants moved to a new screen on the application, when they completed a quiz, and when they interacted with an animated widget. For the FREE-BROwsE condition we logged the browser button actions - such as 'back' and 'home' - along with each individual webpage they visited. In both the COMPANION and FREE-BROWSE conditions we expected to see greater interactions with mirroring methods that allowed participants to better manage their attention. We then used the interview data to frame the quantitive data and explain any anomalies.

\subsection{RESULTS}

We considered a range of objective and subjective metrics to evaluate the participants' experience of the commonality methods. For the Likert scale questionnaires we first conducted Friedman tests to determine if there was a general overall 
effect. If there was, we then ran post-hoc Wilcoxon tests between the conditions to determine any inter-condition effects. We set $\alpha=0.05$ for significance testing throughout our study.

\subsubsection{Post-Clip Questionnaires}

The results for the post-clip questionnaires are shown in Table 13. These are divided into those participants who got the FREE-BROwSE condition, and those who got the COMPANION condition.

When considering taking in information on screens - first considering the comPANION condition, there was a significant effect for the participants' ability to take in the content over two screens (statement a)), with pairwise differences - the participants found it significantly easier for the $C_{2}$ condition $(Z=3.24, p<0.001)$ and the $\mathrm{C}_{4}$ condition $(\mathrm{Z}=1.95, \mathrm{p}=0.003)$, compared to the $\mathrm{C}_{1}$ condition. These results were similar to those participants who had the FREE-BROwSE condition there was a significant overall effect, and $\mathrm{C}_{2}$ was the most preferred method. From conducting pairwise post-hoc tests we were able to determine that $\mathrm{C}_{2}$ was significantly preferred to $\mathrm{C}_{1}(\mathrm{Z}=3.00, \mathrm{p}<0.001)$ and $\mathrm{C}_{3}(\mathrm{Z}=1.789, \mathrm{p}=0.037)$. In addition, the participants felt that they were able to manage their attention across the two screens significantly better for $\mathrm{C}_{2}$ than $\mathrm{C}_{3}(\mathrm{Z}=2.079, \mathrm{p}=0.019)$. 


\begin{tabular}{|c|c|c|c|c|}
\hline & \multirow[b]{2}{*}{ Statement } & \multicolumn{3}{|c|}{ Mean Ranl } \\
\hline & & $\mathrm{C}_{1}$ & $\mathrm{C}_{2}$ & $\mathrm{C}_{3}$ \\
\hline \multirow[t]{7}{*}{ COMPANION } & a) I found that I could take in the content over the two screens at the same time effectively & 2.00 & 3.10 & \\
\hline & b) I had to shift my viewing between the screens a lot to take in the TV and tablet content & 3.08 & 1.93 & 2.3 \\
\hline & c) I found this commonality presentation method visually appealing & 2.63 & 2.95 & 2 . \\
\hline & d) I found the commonality method used got in the way of my content viewing on the tablet & 2.85 & 2.35 & 2.4 \\
\hline & e) I found that the commonality method got in the way of viewing the TV material & 2.35 & 2.08 & 2.7 \\
\hline & f) I felt I had good awareness of what was happening on the TV while looking at the tablet & 2.33 & 2.58 & 2.8 \\
\hline & g) I felt I had good awareness of what was happening on the tablet while looking at the TV & 2.50 & 2.43 & 2.8 \\
\hline \multirow[t]{7}{*}{ FrEe-Browse } & a) I found that I could take in the content over the two screens at the same time effectively & 2.15 & 3.43 & 2.6 \\
\hline & b) I had to shift my viewing between the screens a lot to take in the TV and tablet content & 3.20 & 1.73 & 2.0 \\
\hline & c) I found this commonality presentation method visually appealing & 2.63 & 3.28 & 2.4 \\
\hline & d) I found the commonality method used got in the way of my content viewing on the tablet & 2.43 & 2.53 & 3.0 \\
\hline & e) I found that the commonality method got in the way of viewing the TV material & 2.55 & 1.65 & 2.4 \\
\hline & f) I felt I had good awareness of what was happening on the TV while looking at the tablet & 2.15 & 3.28 & 2.7 \\
\hline & g) I felt I had good awareness of what was happening on the tablet while looking at the TV & 2.60 & 2.75 & 2.2 \\
\hline
\end{tabular}

Table 13: This table denotes the extent to which the participants agreed with the not Strongly Agree (5); to Strongly Disagree (1). Larger numbers are indicated with darker co ranks and the $\chi^{2}$ value, and statistically significant $(p<0.05)$ are denoted in red - with $i$ at higher significance levels. It is clear from observation of the number of statistically that the FREE-BROWSE condition results in a larger effect for many conditions. 
Now turning to the perceived switching cost of the users - the COMPANION scenario, there was a significant overall effect for switching cost across conditions (statement b)). Compared to the baseline $\left(\mathrm{C}_{1}\right)$, all conditions were significantly better at reducing switching cost $-\mathrm{C}_{2}(\mathrm{Z}=2.70, \mathrm{p}=0.004) ; \mathrm{C}_{3}(\mathrm{Z}=1.94, \mathrm{p}=0.027) ; \mathrm{C}_{4}$ $(\mathrm{Z}=1.65, \mathrm{p}=0.026)$. In general $\mathrm{C} 2$ was ranked consistently the highest, and ranked significantly higher than the screen-in-screen on the TV case $-C_{4}(Z=1.949, p=$ 0.026). This effect was also observed in the FREE-BROwSE condition, with a much larger effect than the companion case - (COMPANION $\chi^{2}=11.86$, FREE-BROWSE $\chi^{2}=$ 27.16). For the case in which we mirrored from the tablet to the corner of the TV in the FREE-BROWSE case, there was a significant difference between both the screenin-screen on tablet $\mathrm{C}_{2}(\mathrm{Z}=3.22, \mathrm{p}=0.005)$ and the condition in which we mirrored the TV content behind the tablet's browser $\left(\mathrm{C}_{3}\right)(\mathrm{Z}=3.131, \mathrm{p}<0.001)$.

In terms of visual appeal there was no significant difference for the COMPANION condition in terms of visual appeal for the conditions (statement c)). However, for the FREE-BROWSE condition, there was an overall significant effect for the conditions. In general, $C_{2}$ was preferred significantly more than $C_{1}(Z=1.724, p=0.034)$ and $\mathrm{C}_{4}(\mathrm{Z}=2.411, \mathrm{p}=0.008)$.

For tablet occlusion, there was no significant effect in the COMPANION condition for the commonality methods occluding the tablet content (statement d)). However, for the FREE-BROWSE condition it was evident that $\mathrm{C}_{3}$ - the condition where the video was placed behind the tablet content caused more interference with the web browser $\mathrm{C}_{4}(\mathrm{Z}=2.153, \mathrm{p}=0.016)$.

When considering television material occlusion - for the COMPANION study there were no significant effects for perceived occlusion of the TV material (statement 
e)). However, it was evident that for the FREE-BROwSE study, the participants found that the placement of the tablet on the TV affected their perceived occlusion of the TV.

Now, in terms of the participants' awareness of the tablet content there was no significant effect observed for the COMPANION condition (statement $\mathrm{f}$ )). However for the FREE-BROWSE case there was a significant effect. Significant pairwise differences were observed between the baseline $\left(\mathrm{C}_{1}\right)$, which was perceived as worse than $\mathrm{C}_{2}(\mathrm{Z}=3.363, \mathrm{p}<0.001)$ and $\mathrm{C}_{3}(\mathrm{Z}=1.65, \mathrm{p}=0.049)$. In addition it is possible to see that $C_{3}$ was rated significantly worse than $C_{2}(Z=1.854, p=0.032)$ and $\mathrm{C}_{4}(\mathrm{Z}=2.052, \mathrm{p}=0.020)$. Finally, as with tablet awareness there was no major effects for the amount to which the users were aware of the TV (statement g)). And, in contrast to the participant's reported tablet awareness we did not see any significant effects for the FREE-BROWSE case.

\subsubsection{Post-Study Questionnaires}

We conducted analysis of the post-study questionnaires presented to the participants, the results of which are shown in Table 14. With regards to perceived mental effort, a significant overall effect for the COMPANION content condition was observed. Upon conducting post-hoc analysis of the data it was evident that the video content in the corner of the tablet $\left(\mathrm{C}_{2}\right)$ resulted in the participants experiencing less mental effort than the baseline case $\left(\mathrm{C}_{1}\right)(\mathrm{Z}=3.096, \mathrm{p}=0.001)$. In addition, there was a similar, but not as pronounced effect, for when casting the tablet content on the $\mathrm{TV} \mathrm{C}_{4}(\mathrm{Z}=2.397, \mathrm{p}=0.009)$, and when placing the video content behind the tablet material $\left(\mathrm{C}_{3}\right)(\mathrm{Z}=1.911, \mathrm{p}=0.028)$. 


\begin{tabular}{llccccccc}
\hline & \multicolumn{5}{c}{ Mean Rank } & & \\
\cline { 3 - 7 } & \multicolumn{1}{c}{ Statement } & 1 & 2 & 3 & 4 & $\chi^{2}$ & $\mathrm{p}$ \\
\hline \multirow{2}{*}{ COMPANION } & a) The UI for condition $N$ required mental effort to view both screens & 3.15 & 1.93 & 2.53 & 2.40 & 11.18 & 0.011 \\
& b) I liked clip $N$ & 2.48 & 2.23 & 2.60 & 2.70 & 2.06 & 0.56 \\
FREE-BROWSE & a) The UI for condition $N$ required mental effort to view both screens & 3.23 & 1.63 & 2.50 & 2.65 & 19.34 & 0.000 \\
& b) I liked clip $N$ & 3.10 & 1.75 & 2.50 & 2.65 & 14.17 & 0.003 \\
\hline
\end{tabular}

Table 14: Post-study questionnaire: the extent to which participants agreed with a given statement. $N$ refers to the particular number of the clip, or condition. It is clear that, when reflecting on their experiences, the participants saw major differences between the conditions in terms of their attention.

There was a similar, but more pronounced difference for the FREE-BROWSE case, where all conditions were ranked significantly lower for perceived mental effort than the baseline: $\mathrm{C}_{2}(\mathrm{Z}=3.223, \mathrm{p}<0.001) ; \mathrm{C}_{3}(\mathrm{Z}=2.303, \mathrm{p}=0.010) ; \mathrm{C}_{4}(\mathrm{Z}=2.236$, $\mathrm{p}=0.013)$. In addition the TV mirrored in the top of the tablet, $\mathrm{C}_{2}$, was also ranked significantly better than when the video was mirrored behind the web browser, $\mathrm{C}_{3}$, $\mathrm{Z}=2.797, \mathrm{p}=0.0025)$ and when compared to when mirroring the tablet content to the TV $\left(\mathrm{C}_{4}\right)-\mathrm{Z}=2.753, \mathrm{p}=0.003$. Finally, With regards to the clip preference it was clear that the participants least favourite clip was Clip 2 (statement b)). Interestingly, there was a significant effect for how much the participants enjoyed each clip for the FREE-BROWSE, but not for the COMPANION clips.

\subsubsection{Device Log Data}

As shown in the bar chart in Figure 27 we saw, in general, that $\mathrm{C}_{2}$ elicited more interaction than any other condition. There was a significant overall effect between the four conditions $-(\mathrm{F}(3,17)=3.314, \mathrm{p}=0.45)$. We then conducted post-hoc tests and determined that the case in which the participants had the TV screen mirrored into the corner of their device, $\mathrm{C}_{2}$, elicited significantly more interaction than the 
baseline case, $\mathrm{C}_{1}(\mathrm{Z}=3.104, \mathrm{p}=0.002)$ and when mirroring behind the tablet content $\left(C_{3}\right)(Z=2.296, p=0.022)$. In addition, as shown in Figure 27, we conducted analysis of the number of times that the users interacted with the animation and found this was consistent across all conditions, with no significant effect.

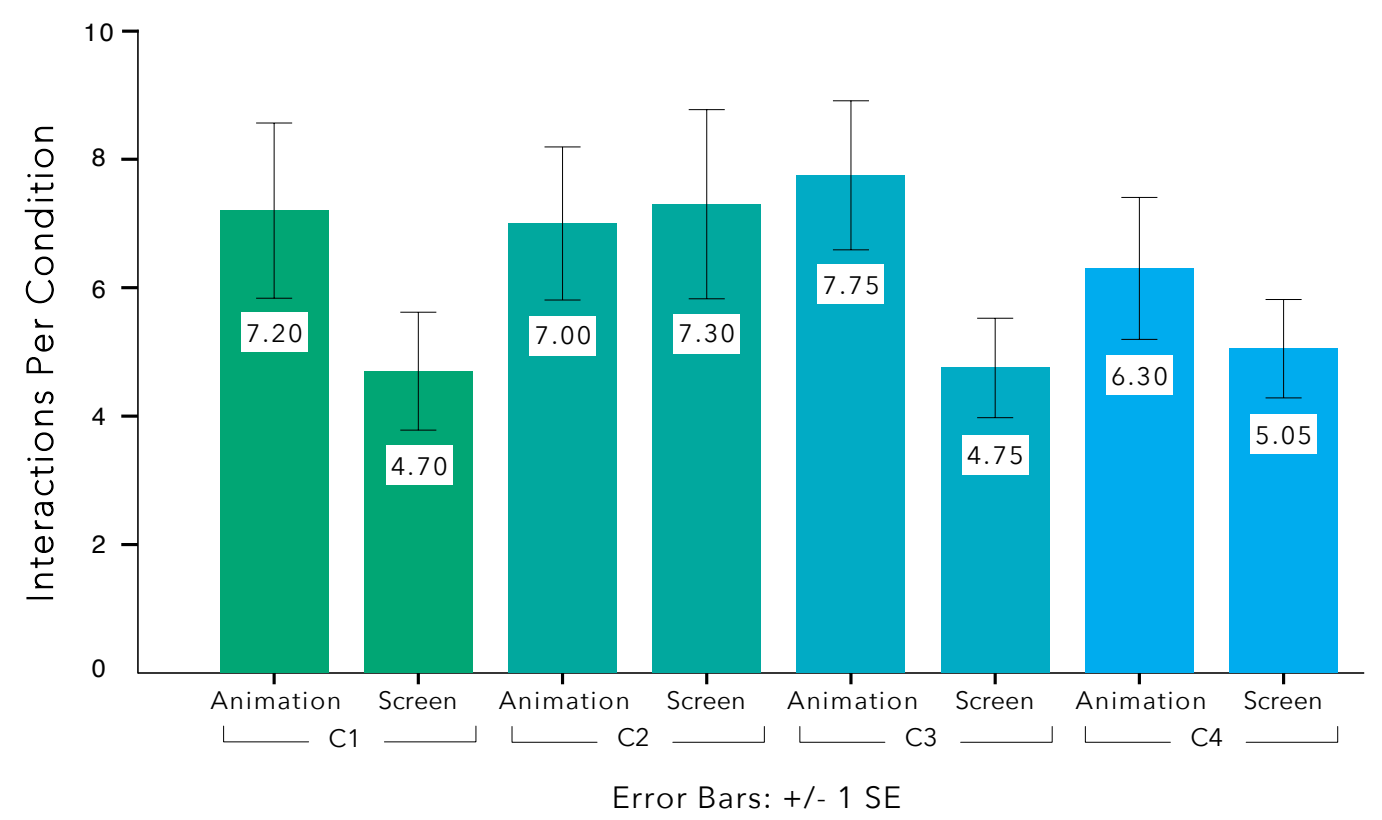

Figure 27: Mean interactions per condition, per participant: this graph describes the average number of each type of interaction per condition. Note that users were much more likely to move screen when in the TV was mirrored into the corner of the tablet screen - $\mathrm{C}_{2}$.

Now, turning to the FREE-BROwSE condition - it was clear that different commonality methods appeared to have an effect on the participants' browsing of the internet $(\mathrm{F}(3,17)=3.871, \mathrm{p}=0.028)$. As shown in Figure 28, it is clear that when the participants had the TV in the corner of the tablet screen $\left(\mathrm{C}_{2}\right)$. From conducting post-hoc analysis we were able to determine that the users were significantly more active browsers when searching the internet with the TV mirrored in the corner 
of the tablet $\left(\mathrm{C}_{2}\right)$ compared to the baseline $(\mathrm{p}=0.039)$ and when compared to the video underlay condition $\left(\mathrm{C}_{3}\right)(\mathrm{p}=\mathbf{0 . 0 2 2})$.

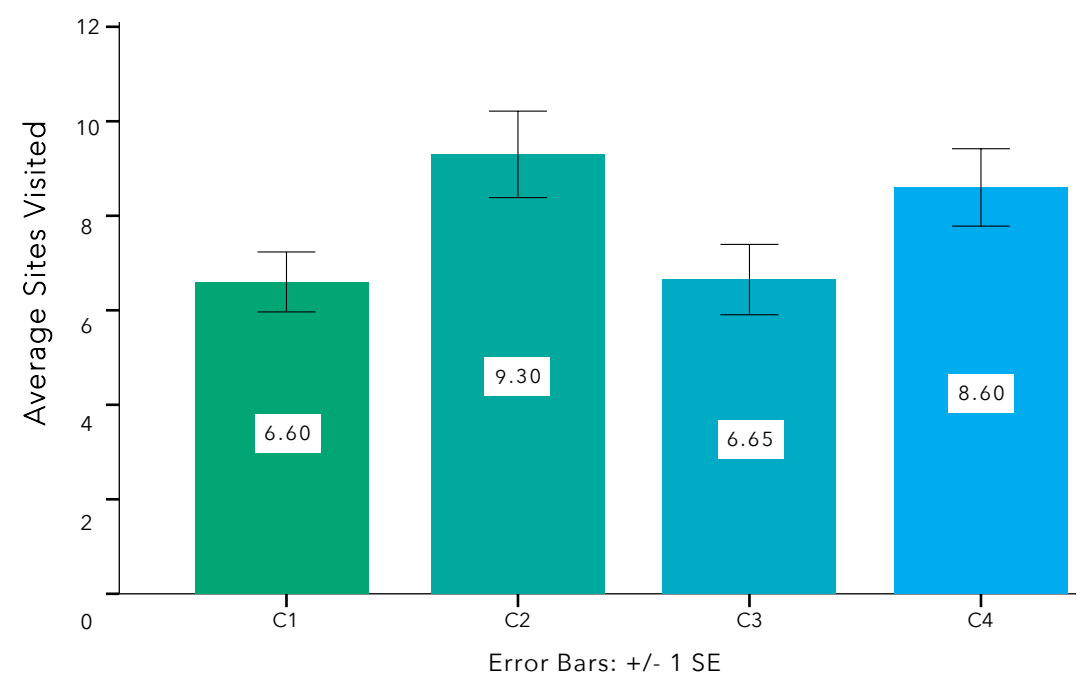

Figure 28: Mean number of sites visited per session, per participant: here we can see that when we mirror from the TV to the mobile device, it stimulates more viewing.

\subsubsection{Post-Study Interviews}

In general, the participants enjoyed the methods in which the tablet mirrored the content from the TV to the tablet. For example, to the initial question, which asked if the participants found that any of the methods were useful for taking in the content across the two screens $\mathrm{C}_{2}$, was noted as the preferred method over half of the time, with $24 / 40$ stating that this method helped them take in the content over two screens $-10 / 20$ for the COMPANION content study and 13/20 for the FREE-BROWSE (for a total of 23/40). The case in which the TV content was mirrored under the mobile device content, $\mathrm{C}_{3}$, came second in terms of preferences - 12/40 participants favouring this option. Mirroring to the TV was not regarded as popular -4 out of 
40 participants preferring this condition. Only one participant out of 40 said that the commonalities did not help them.

Regarding the participants' reasoning for their preferences - many of those who enjoyed the content mirrored from the TV to the tablet appeared to do so because it allowed them to get a gist of what was happening on the TV, meaning that they could better choose when to look up to view the TV content - "I could see this thing [the mirrored TV content] in the corner of the screen and if there was something I thought was really interesting and I wanted to see in full view, I could just look up at the TV. I felt like I was in control" $\left(\mathrm{P}_{34}\right)$ - "My preference is having the small screen in the corner of the device - you sort of then look at the TV if you see that something interesting is going to happen on it in more detail" $\left(\mathrm{P}_{39}\right)$.

When comparing $\mathrm{C}_{2}$ and $\mathrm{C}_{3}$, the techniques which mirrored the TV on the tablet, the participants tended to prefer $\mathrm{C}_{2}$ because of the way it occluded the tablet content - i.e., $\mathrm{C}_{2}$ fully occluded a small part of it, instead of partially occluding all content, as $\mathrm{C}_{3}$ does. This trade-off between size and opacity was something that the participants discussed extensively; for instance, $\mathrm{P}_{2} 6$ preferred $\mathrm{C}_{3}$ as it allowed for better resolution for the video content - "I think it was easier to both watch the show and google at the same time, because of the overlay - you weren't losing any size of screen." ( $\left.\mathrm{P}_{35}\right)$. Many participants who preferred $\mathrm{C}_{2}$ noted that even though the mirrored video was small, because they were using it as a cue when to look up at the full size picture on the TV this wasn't a major concern, as indicated by $\mathrm{P}_{22}$ - "It was handy - didn't feel it got in the way of anything. And sometimes, as it was a little small, for the more visual aspects I would look up to see what was going on.” 
Participants also noted differences in the way that they experienced their eyes shifting around the tablet screen between the mobile device intra-screen conditions - $\mathrm{C}_{2}$ and $\mathrm{C}_{3}$. Some users' comments tended to indicate that they preferred $\mathrm{C}_{3}$ because it allowed the them to monitor the events better on the TV as they did not need to consciously switch their gaze to the tablet computer's corner in their peripheral, but simply change their focus slightly and look 'through' the overlaid content - "...the one that was [mirrored] behind gave me the opportunity to read what was on the screen without having to move my eyes to see what was happening" (P8). But on the other hand they found that the intermixing of the video and the material on the tablet often became confusing, especially for those doing the FREEBROWSE condition - "I couldn't see when I wanted to look at the internet properly it put me off what was going on behind it" $\left(\mathrm{P}_{3} 8\right)$. One participant even found the underlaid video content disorienting to the point where it made them feel slightly nauseous - "yeah there was a big swooping shot and I was like *blergh" - P21. One participant noted a limitation of $\mathrm{C}_{2}$ may be the fact that as well as fully occluding an area, it may totally block off interface elements with some applications if not thought out properly - "I thought that it was quite useful, but if you need to interact with anything in the top corner - like to close an app or something - you can't." ( $\left.\mathrm{P}_{30}\right)$.

Participants generally did not respond as positively when the content from the tablet was mirrored onto the TV $\left(\mathrm{C}_{4}\right)$. Generally, this was because they believed it to be occluding the larger screen - "it got in the way when I was watching the $T V$ " ( $\left.\mathrm{P}_{1}\right)$. Also, participants often noted that, as it was on the TV, it did not afford reading text - "I didn't like that so much because the writing was smaller and I found it easier to read when it was right in front of $m e "\left(\mathrm{P}_{2}\right)$. Another concern from the 
participants was typing - as they were using a non-tactile (tablet) keyboard, this meant that they had to regularly look down at the tablet to type regardless, for example, P29, who had issues typing in queries on the second screen and looking at the TV, just resorted to switching back to the tablet regardless - "I couldn't type on the tablet and look up. I couldn't move back and forth between the screens".

The 'default' condition, without any commonalities - the one all second screeners currently experience in their day to day viewing - was the least preferred according to our data. Our interviews suggest that this is due to perceived switching cost and the mental load of the simultaneous information streams - for example P14's comment - "...and the most difficult was the last one $\left(\mathrm{C}_{1}\right)$ because I had to check the $T V$ and the tablet". In fact, only one participant (P18) noted disliking the commonality methods as they were not familiar with their regular second screen routine "In the beginning, it's a little overwhelming because you're not used to watching this way. But actually, you're used to doing the 4 th method (C1)".

\subsubsection{Discussion}

It was clear from the post-clip questionnaire that the commonality methods appeared to help the participants take in the information across the two screens, to varying degrees. Mirroring from the TV into the corner of the tablet $\left(\mathrm{C}_{2}\right)$ appeared to be the most effective method for the COMPANION and the FREE-BROwSE case. Moreover, the methods in which the TV was mirrored to the tablet were considered the most effective. The interview data suggests that $\mathrm{C}_{3}$ did not perform as well as $\mathrm{C}_{2}$ because of the way that it visually clashed with the video content. Evidently, this was more predominant in the FREE-BROwSE case - where the visual content was dictated by the user freely browsing the web. As one would expect, 
however, there were some strong inter-participant and inter-content variances due to differences in personal preferences.

The commonality methods also appeared to reduce the perceived cost of the participants moving their focus from one display to another in both the COMPANION and the FREE-BROWSE condition. The qualitative interview data suggests that the participants were switching their focus intra-device, and then making an active decision to switch their focus when they wanted - empowering them to be more in control of their own attention, in contrast to say, the broadcaster driving the experience, as discussed in the previous chapter.

Here, the screen-in-screen method on the tablet $\left(\mathrm{C}_{2}\right)$ resulted in significantly lower switching cost than when replicated on the TV $\left(\mathrm{C}_{4}\right)$. This finding is likely because the TV updates faster than the tablet, and therefore the method enabled the participants to monitor the events on the TV, and to look up when their attention was caught by the visuals of the TV in the corner, or when something in the audio could not be clearly inferred from the small screen in the corner of the tablet.

When checking to determine how aware the participants were of the tablet material, we expected that the method in which we mirrored the tablet content to the TV would improve this significantly compared to the baseline (no commonalities), which it did. However, what we did not expect was that, when browsing the web, the participants appeared more aware of the tablet content for the two methods which mirrored content from the TV to the tablet. This, as indicated by the interview data, is likely because they did not have to look up at the TV as much to fill in the gaps in their (visual) perception, essentially meaning they were, as a result of this, more focused on their web browsing experience. These tablet awareness 
effects, however, were only seen in the FREE-BROwSE, and not the COMPANION condition. A potential explanation here is that it is simply the amount of information on the web browser, and the cognitive effort associated with knowledge query which exacerbated this effect, as shown in Chapter 5 .

Evidently, the log data shows that the participants navigated around the application much more when in the condition in which we mirrored the TV in the corner of the mobile device. In light of the subjective quantitative and qualitative data, this is likely because they experienced the least mental demand in this scenario, and therefore were able to engage more with the application. The animation, however, did not show this pattern. The log data suggests that this is because most participants went through the transitions in the animation, and just stopped at the final one for each - resulting in quite uniform inter-condition data.

This propensity to search for more information when the TV was mirrored onto the tablet was also reflected in the amount of web browsing the participants did this is likely the same effect being exhibited - as the participants can easily monitor the TV in the app, they were able to engage with it more. This effect, interestingly, was not seen when the browser was placed over the full mirrored TV screen on the tablet. The interview data indicates that this may be to do with the perceived issues some of the participants faced in this condition due to the two streams of content clashing.

\section{6}

IMPLICATIONS, FEASIBILITY AND THE FUTURE

Our investigation has suggested strongly that methods in which material is mirrored from the TV to a mobile device can be of significant benefit to users in mitigating 
the attention constraints of multi-device media. This was particularly noted when mirroring from the TV to a tablet in a screen-in-screen method. In addition, it is clear that other methods, such as placing a video stream behind content on a mobile device, are also useful technique, most beneficial to scenarios in which the content on the mobile device can be designed around this - e.g. companion content scenarios. We, therefore, suggest that, in general, to improve the experience of such applications, designers should consider using techniques that use such duplication of common elements, in order to reduce the effort required in viewing companion applications or similar material.

Though we envision a future in which both persistent and non-persistent duplicate mirroring can be easily integrated into cross-device media scenarios, further research is still required. Our studies, for example, have not explored the effect of persistence: all of our commonality methods were constant and the participants were not able to, for example, dismiss the duplicated video from their attended device. Future prototypes, such as that shown in Figure 29 will transcend this constraint, and allow users to control which mirroring method they employ in their browsing. Future work could then observe, longitudinally, and appropriation of mirroring methods by users in their everyday viewing.

Such investigations, however, would open up new problems, questions and areas for design. The further investigation of such methods on a variety of devices, for example, would be of considerable interest as a next step. By exploring diverse form factors, and screen sizes of everyday mobile devices we can explore the transferability of commonality methods across device types. With smaller devices, such as smartphones and phablets, there are likely to be bottlenecks in the effectiveness of the techniques, leading to new design requirements. For example, the screen-in- 

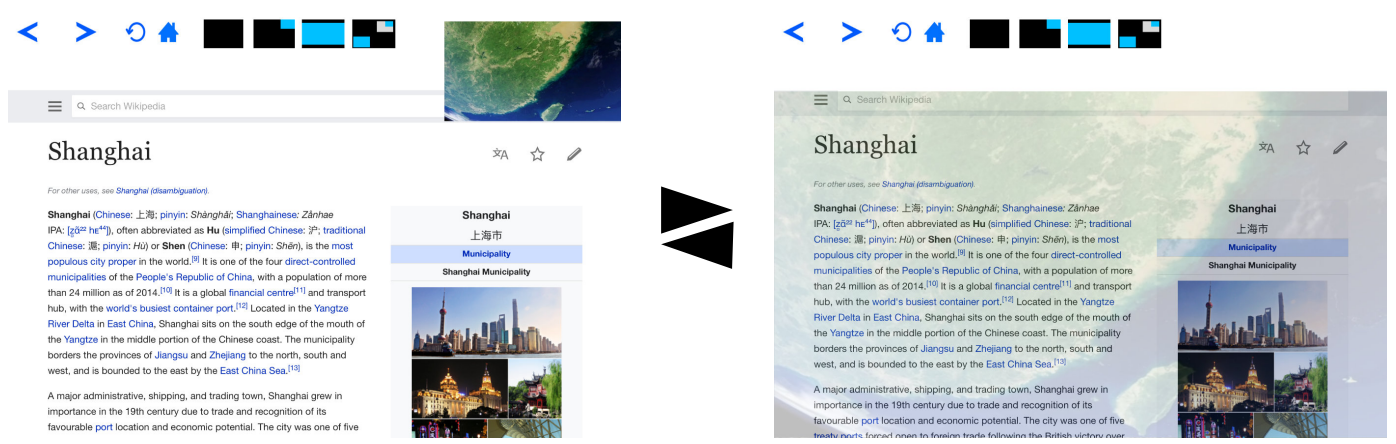

Figure 29: This figure shows a demonstrator application to explain this research at the British Science Festival. The icons at the top allow the user to seamlessly switch between any of the tested UI layout types.

screen condition in our experiments $\left(\mathrm{C}_{2}\right)$, is likely to require adaption to mitigate against occlusion of the materials under the video content. In such cases, methods such as $\mathrm{C}_{3}, \mathrm{C}_{4}$ (and indeed methods not discussed in this chapter) may begin to become more beneficial to users.

Thinking beyond the second screening scenario, the ideas and findings in this chapter have implications for other use cases: for example, we could extend current mobile device EPGs by integrating the feed from the TV into the corner of the screen, allowing a user to choose a new programme while still retaining awareness of the content running on the television screen. Designs such as those proposed in this chapter may assist designers in overcoming the the issues with eyes-off interactions with non-tactile surfaces (e.g., tablets) when engaging with EPGs.

Finally, in terms of the infrastructure to enable such experiences, the barrier to entry is still quite high. It is assumed that our users have a Smart TV, devices connected to it, and a seamless and uninterrupted internet connection. Having such infrastructure in our homes, however, is clearly an accelerating trend, and as more 
of us adopt to, and become familiar with, fast internet-enabled home networks the opportunities for multi-device experiences are only going to increase. With recent developments in the protocols of cross-device interaction - for example the BBC's recent work on Frame-Accurate multi-device content [141] - the possibilities for these cross-device scenarios entering our living room is growing. Propelled by the apparent strong demand of users to have more engaging multi-device experiences, there is great potential for interaction designers and researchers to enhance the understanding and impact of this design space.

\subsection{CONCLUSION}

This chapter has explored display commonalities - the concept of duplicating elements of a user experience over multiple screens, in order to lower the cognitive effort required to engage with dual-screen material. Through discussions with professional content designers, we refined our initial design space and conducted an empirical investigation of the proposed techniques under the two prominent scenarios in this use case - web browsing and companion content.

Regarding the research questions, there was clear evidence in this chapter that the addition of duplicate mirroring reduce the requirement to switch screens unnecessarily and that it allowed the users to take in the content across the screens more effectively, providing additional insight to $\mathrm{RQ}_{3}$. Regarding $\mathrm{RQ}_{4}$, we saw evidence that many of the users were, in conditions in which the content was mirrored from the television to the mobile device, looking at the unattended mirrored screen and then making an active decision to switch their attention; implying that this method afforded additional autonomy, which users took advantage of. 
By analysing the objective and subjective data we have been able determine the efficacy of the proposed techniques. In general we found that, by duplicating the video of the television on a handheld device we can, by extension of currently technology, significantly reduce the required level of cognitive effort require to engage with dual-screens. The findings and design concepts proposed in this chapter, which were explored from a designer and a user perspective, can better equip content creators and developers to overcome the disjuncts in visual attention that exist in multiple screen scenarios and, therefore, improve the UX of cross-device media. 



\section{CHAPTER NINE}

\section{Conclusions and Future Work}

From inception, this thesis has argued for the explicit design of cross-device attention. Our work has aimed to understand and to orchestrate user focus as a design lever to overcome users' mental overload, with a goal of towards fostering harmonious cross-device experiences. Second screening, a ubiquitous manifestation of the multi-device world we inhabit, involves high levels of attention resources, and throughout this thesis we have investigated factors which affect the distribution of attention and how they can be used to enhance such experiences.

In the early stages of this work (chapters 1 and 2) we outline a clear area of investigation from the literature. The literature on attention in this cross-device scenario reports extensively on attention issues around cross-device media, without offering any solutions per se. We therefore, throughout this thesis, have focused on better understanding, and designing for, this scenario.

We began the contributing chapters of this thesis by first understanding the attention bottlenecks of this use case through a large scale survey and in-depth interviews with second screeners. We found that although some specific factors, such as genre of programme, are clear indicators of cross device complexity, bringing us to probe deeper into our understanding of cross device complexity. To this end, we conducted a study to empirically investigate the effect of varying complexity on a mobile device. This led us on to consider another focus of this thesis - intervening 
in attention, for example, altering the complexity of a mobile device (Chapter 6) or through additional stimuli (Chapter 7 ).

For these interventions, we explored additional methods to affect the way in which users distribute attention across devices, focusing on minimally invasive extensions to prior existing technology; evaluating them comprehensively and looking at the tradeoff between content creator and user control. Our results, for each intervention, frame where such techniques may be useful to practitioners. Many of the interventions presented in this thesis were more effective than regular viewing for attention management, i.e. the systems which users currently engage with, in terms of subjective preferences and objective measures of preferred attention distribution. We now outline, in more detail, the specific contributions of this thesis, noting what designers and the TV HCI community may glean from our research.

\section{1}

CONTRIBUTIONS AND REFLECTION ON RESEARCH QUESTIONS

In the Background (Section 2) we first outline the drivers behind the popularity of the second screen use case, describing the academic research around its rise to prominence. We then explored the attention-based literature around the focus of this and outline a number of shortcomings. With this, in Chapter 4 we then progressed to conduct two studies to better understand attention for second screeners from the users' perspective.

Results from our online survey suggested that the addition of a second screen to participants' television experiences adds significant additional mental effort. 
Moreover, for certain genres constant attention is required required attendance to understand nuanced concepts and details.

Further, second screen interactions which required knowledge query generally led to programme disruption. We outlined the effects of genre and the cautionary tales around designing for cross device media, continuing by exploring user reflections deeper through interviews. In this study we explored the myriad ways the mobile device is transforming even the most linear of television experiences. In this chapter our primary contributions are:

- A more complete understanding of the effect of programming genre on second screening;

- A categorisation of the main patterns around users accounting for missed content: mitigating and compensating;

- An overview of the 'types' of second screening we encountered, towards the creation of personas designers may utilise.

In terms of the research questions of this thesis, outlined in Chapter 3 , we begin to answer RQ1, in that we outline many of the main issues that face users when engaging with mobile devices. Our findings, here, bolster the need to consider TV genre in relation to types of second screen content users engage with. In addition, we outline the ways in which users overcome the issues (mitigating and compensating) and outline some potential strategies to support these behaviours (e.g., allowing pausing and rewinding with synchronised in-app content).

In Chapter 5 we progressed from understanding the current experience of second screening to further consider how we may better understand cross-device com- 
plexity, with a view to create more appropriate second screen material. By conducting experiments with television programmes and time synchronised second screen material of varying complexity, we explored the effects of varying textual and graphical detail on the experience. Our primary contributions in this chapter were:

- An empirical investigation of what constitutes 'complex' second screen content from an objective and subjective perspective;

- A more detailed understanding of what constitutes complex television material through discussions with users;

- Guidelines for complexity in terms of second screen textual complexity using standardised metrics.

In this chapter we gather more data to understand RQ1 and RQ2 - relating the experienced mental effort to engage with content to specific quantities and complexities of textual and graphical content.

By utilising the results of the users' objective feedback on the characteristics of complex TV content from Chapter 5 , we further considered how textual complexity (a clear driver of cross-device attention) may be varied by an algorithm (an adaptive UI), or by the user (an adaptable UI). We conducted an empirical user study to evaluate the effect of the adaptive and adaptable UIs with users with a view to better answer RQ3. From our studies we found that: users experienced the adaption, despite not being informed of its presence before the trials; and that, in general, they strongly preferred the materials to be curated for them, over a randomised baseline or them adapting the materials. 
The evidence suggests that our methods afforded a better cross-device experience as they were able to better take in the content across the two screens. Complexity adjustment afforded a more passive 'sit back' experience, when the adaption was fully driven by the device, and provoked some participants to become more active and take more control in the way they viewed the content by adjusting the complexity. This chapter offers a number of key contributions, namely:

- a proposal and investigation of adaptable and adaptive content in the context of variably textual complexity on a mobile device while second screening;

- a validation of our heuristics, derived in the previous chapter, for determining what constitutes 'complex' video material;

- deeper insight into how our findings from the previous chapter relate to textual complexity and the viewer experience.

This chapter showed that it is possible to intervene in users' attention to improve the way they manage it $\left(\mathrm{RQ}_{3}\right)$, and supported the results of the previous chapter, aiding the understanding of RQ2. Further, we began to explore $\mathrm{RQ}_{4}$ - the extent to which a user should be in control of their own attention management. The two interventions in this chapter afforded high user-autonomy (an adaptable interface) and low user-autonomy (an adaptive interface). There was evidence supporting both high and low autonomy - some users preferred to sit back and have the content presented to them (adjusted to the television), and others wished to go in search for more content to find out more (more/less information buttons). In the next chapter, we investigated $\mathrm{RQ}_{4}$ deeper by exploring what happens when a user is directed and is given low autonomy over their own experience. 
In Chapter 7 , instead of changing the material, we explored how content creators can create shifts in attention to a given device at a key moment in a broadcast experience. We conducted an experiment in which we embedded auditory and visual stimuli within cross-device media. Our data suggested that to attract attention quickly to an unattended device, we should consider auditory methods, especially abstract sounds (earcons).

To allow users to defer when they switch their attention, we should inform them visually, on the attended device. Our findings suggest that such our approach mediating attention - helped users break the innate tension in not knowing when to look at a given screen, aiding them in not missing material or frequently checking for updates. We frame our results as a series of potential design levers for those wishing to design cross-device experiences. Our key novel contributions, here, are:

- a proposal, and an empirical investigation of, additional stimuli in crossdevice media;

- strong evidence suggesting that users prefer their attention to be driven by some additional stimuli;

- a framework for designers to evoke shifts in attention between devices at given times and urgencies.

Regarding the extent to which the user should be in control of their own experience $\left(\mathrm{RQ}_{4}\right)$ we found that this low user-autonomy method of directing their attention allowed the participants to overcome may of the issues of multi-device content experience, for example, where to look at what time and how not to miss 
relevant content. In general, mediating attention with additional audio-visual stimuli was found to be a highly effective and preferable method for intervening in users attention, despite their lower autonomy in the experience (further answering $\left.\mathrm{RQ}_{3}\right)$.

Our final contributing chapter, Chapter 8, explores methods by which we may utilise duplicate elements - which we term display commonalities - of a user experience across multiple screens so that users may better adjust their own attention, to reduce the cognitive effort and switching cost associated with multi-device experiences. We first outline a design space, whittling this down with discussions with professional UX designers and a creative director at the BBC.

We then conduct a large-scale empirical investigation in a companion content, and standard web browsing condition. Our results suggest that, through duplication of video from a television on a handheld device, we may significantly lower the amount of mental effort required to engage with a dual-screen experience, for both a free-browsing and a companion content case. The techniques and design space in this chapter offers a new perspective on screen mirroring - by mirroring unattended elements within an attended display we better blend the digital worlds - allowing users to feel they require less mental effort and are required to switch attention less. In our final contributing chapter, the key novel contributions are:

- a mirroring concept for cross-device interaction - to incorporate duplicate elements as a design lever to reduce switching cost and attention required to engage;

- insight from designers into mirroring with regards to the feasibility of screen mirroring in cross-device content; 
- an empirical investigation of display commonalities, showing a clear benefit for duplication of television content on a mobile device.

The main goal of exploring this display technique was to explore giving the user additional autonomy of their experience (further answering RQ4). In contrast to the previous chapter on mediating attention, this chapter involved a high-autonomy technique - essentially giving the user the ability to better manage their attention by providing unattended content on attended devices. Our evidence suggests high autonomy cam also be an effective method of attention management in crossdevice scenarios.

\subsection{A GENERIC FRAMEWORK FOR DESIGNING INTERVENTIONS}

The contribution this thesis makes is a more thorough understanding of the way that users engage with their devices, and a series of tools to assist content developers and application developers to manage cross-device attention. However, beyond this, an over-arching and extensible contribution of our work is our methodology. We have followed a user-centred design methodology to understand and design interventions for this context, taking insights from our studies into actionable preventative measures and interventions. Our methodology impacts on three main parties: those who study the behaviours around technology; those who create cross-device experiences; and the technology-level media content developers, who ultimately develop the applications.

In Figure 30, we describe the generic framework we have explored in this thesis, towards enabling optimum attention distribution for users. At the bottom of the hierarchy is its most fundamental component; understanding the problems users 


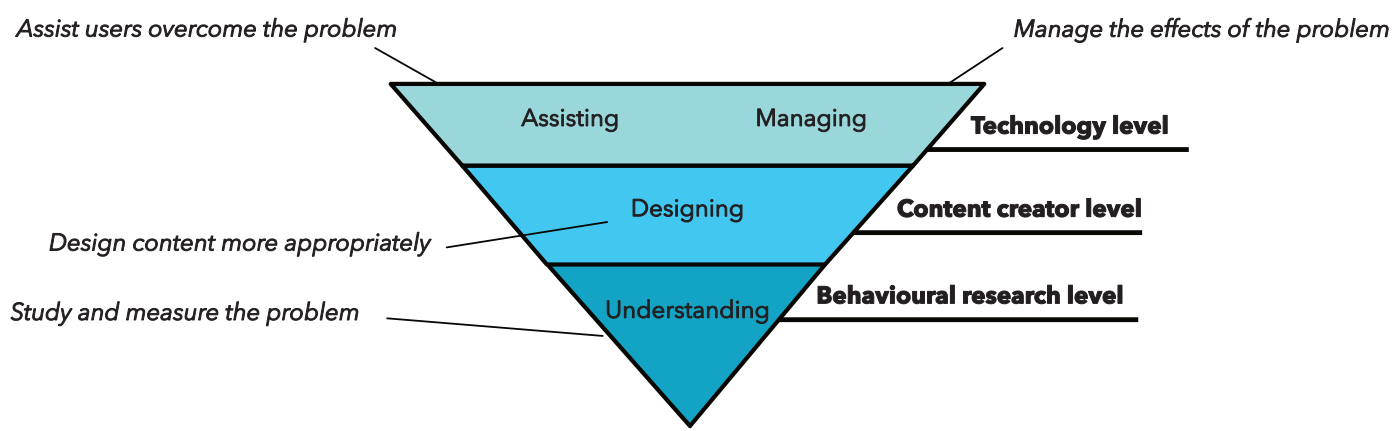

Figure 30: A Bottom-up hierarchy of designing attention management techniques.

have around managing their attention. Throughout this work we explored this through online questionnaires, interviews and empirical studies of attention distribution. This is work that lends itself to the techniques of behavioural scientists and initial qualitative probes, such as interviews and questionnaires.

Up a level, we use this information to prevent the issues around attention management, for example, in our work by controlling the complexity of the material (complexity curation). Curating the materials is arguably the job of a content creator where, guided by some insight about the effects of certain content (for example, the effect of textual complexity), the content creator reaches some active decision about the complexity of the material they provide.

Finally, there is a divergence - one can either assist users in managing their own attention issues, or manage it for them in some way. These are technology-level interventions, which may be embedded into cross-device experiences to reach the intended goal. We can allow users to manage their own attention by giving them a 'crutch' to rely upon to prevent the issues. For example, we allow them to adjust the complexity in some way, or more quickly attend the unattended display (see Chapter 8). As for managing the users' attention, we showed that this is possible 
by mediating their attention with notifications, influencing them to attend certain information at at given time.

This framework has not only allowed us to design interventions to positively affect the way users engage with dual-screen experiences, but it can also be applied to deeper considering the issues around this use case, and assist others in designing attention. Ultimately, after considering methods to improve cross-device attention issues after preventing (Figure 30), one must consider whether the situation is more fit for interventions which assist users in managing their attention, or more direct management. This is dependent on the autonomy required for the experience.

\subsubsection{Autonomy of Intervention}

Throughout our interventions we explored varying degrees of control from the perspective of the content creator and the user $\left(\mathrm{RQ}_{4}\right)$. We illustrate in Figure 31, for example, that general second screening, such as browsing related content, offers high user autonomy. Little to no input is provided from the content creator and the two digital worlds sit in separation. With companion applications, for example, we see more control shift to the content creator, with varying user autonomy of attention dependent on the application. Then finally, increasingly levels of content creator control with methods such as mediating attention, where the broadcaster can shift user attention with varying degrees of urgency, to a given screen.

There is a clear continuum of autonomy for those designing cross-device experiences to consider - the level to which one should engage a user is vital in terms of integrating the work of this thesis into any experience. Our techniques can, 


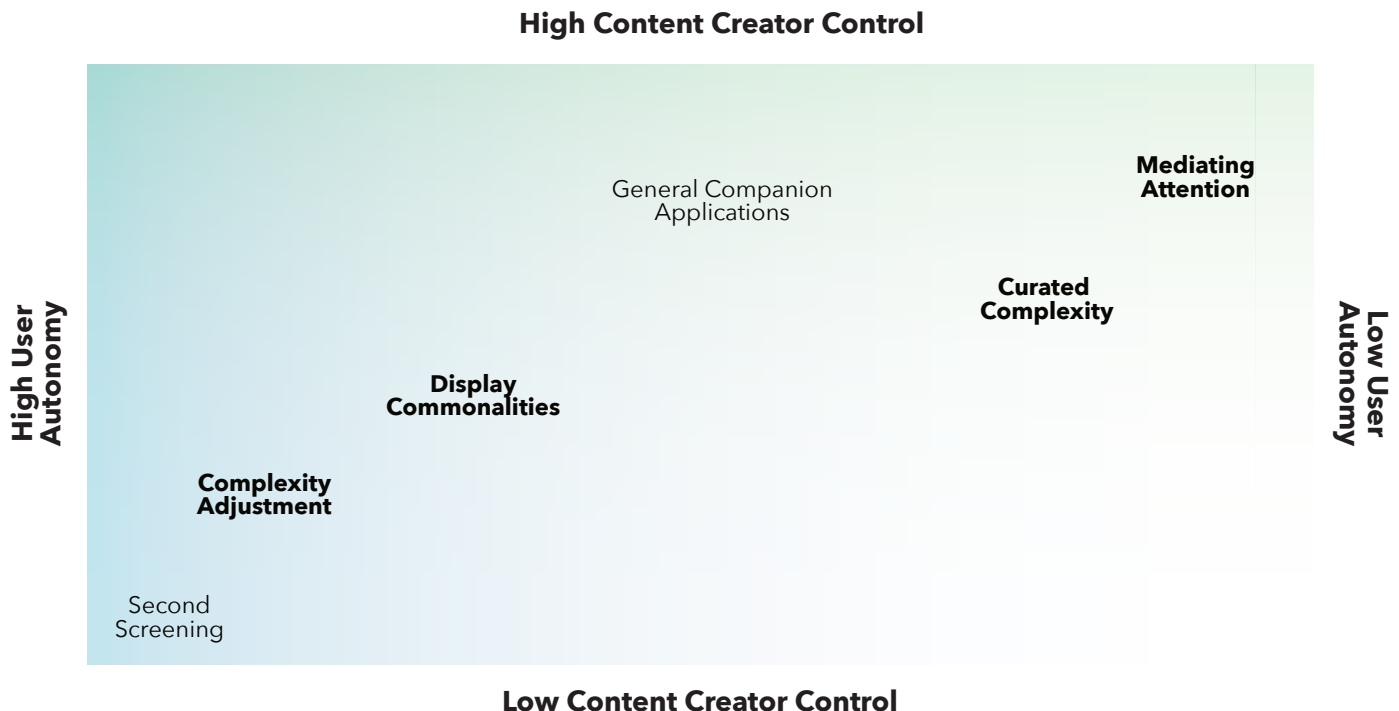

Figure 31: How the interventions, in bold, fit in terms of how much control the user or content creator is given over a user's attention. For example, to second screen requires low content creator control and affords high user autonomy, whereas a content creator mediating a user's attention gives the content creator more control of attention.

therefore, be interchanged to offer varying degrees of autonomy to both content creators and users.

What we present throughout our work in relation to $\mathrm{RQ}_{4}$ ("to what extent should the user be in control of the way their attention is managed in a given context?) is not an answer to the question "what autonomy should one provide?", but a series of techniques to provide varying degrees of user control. Above all, it is vital to note that we found that techniques that provide high and low user control can both be highly effective to assist users in managing the way they engage with multi-device media systems, and that this is highly related to the context of use and the way in which the techniques are implemented. 
Although this thesis has provided strong evidence for our findings and thorough empirical investigations of our interventions, it is important that we define the constraints of our research so that others may develop our techniques, and gain further insight from our work.

With regards to context of use, our investigations of interventions were wholly investigated in a lab environment, either at the BBC or at Swansea University. We focused on using 'second screeners' in our research, however we did not consider them in their native context (their homes). Moreover, the television content we used, though made as broad as possible in each study, was bound by the fact that it was archive footage from the BBC. Newer media paradigms, such as streamed video such as YouTube or Twitch may yield different insights.

In extension to this, our research was guided, and ultimately, bounded by the technology of the time - the uptake of capacitive touch-screen mobile devices in the living room has shaped this research. If conducted in five years, the devices and interactions may differ considerably. Longer term trails with users' personal devices, in the form of a deployment would yield further insight for our findings - most notably, their durability and their robustness to an adapting usage context.

As our research aimed to focus on enhancing cross-device experiences, we mostly concentrated on situations where second screening was related to the television material. In many situations, this is not the focus, however. This was done mostly as in our empirical studies we wished to explored the independent variables at hand. A longitudinal approach to our work would have offered more in terms 
of qualitative feedback and general acceptability, but less data for our specific research questions.

Finally, in terms of our capture of visual quantitive data, we opted for a method of video markup, largely because dual-screen eye tracking is relatively unreliable and invasive. However, such methods would give us more detailed information around gaze and fixations of attention which we could only approximate with our methods. The future will likely offer us more capable methods of dual-screen eye tracking and allow future researchers to better elaborate on our findings.

\subsection{FUTURE WORK}

We believe that while our presented research answers questions and offers solutions, it also asks further questions of its own. There are numerous extensible areas in this thesis. Our work on understanding cross-device visual complexity, for example, could be furthered with an inverse approach. Instead of offering the participant varying levels of textual complexity, we could offer them the opportunity to freely explore on the device, and then measure the perceived objective and subjective effects. Through more detailed analysis of gaze, and by using in-depth thematic analysis of interview data paired with device logs, we could probe deeper into query formulation, text comprehension and the general effects of the multidevice interaction. Such a top-down approach would offer a rich picture of usage, albeit without being able to answer more specific research questions.

Similarly, in Chapter 6 our approach, which used a companion application, could be extended by comparing regular and 'simple' web pages, for example ${ }^{1}$. Such an

1 Simple Wikipedia: simple.wikipedia.org 
approach would allow for the investigation to take place with more user autonomy, while sacrificing control of experimental conditions such as textual complexity.

In our work on mediating attention, in Chapter 7 , we considered how a designer may command attention across multiple devices. Here, we used simple notificationstyle cues in the programme and device's content. We envision a future wherein this is extensible and can be applied creatively by digital content creators, towards Jerry Kramskoy's ambition of Orchestrated Media [83]. Imagine strategically embedding additional audio/visual material into the experience with the express intention of mediating attention, across a multitude of devices. For instance, a rustle in the user's peripheral attention as they watch a nature documentary could hint at unexplored material on their device; and a low-pass filter on the audio of the television, with a dimming of the visuals could suggest more pertinent material on the handheld device.

Our final intervention of the thesis, which explored display commonalties, could benefit from a longitudinal study. To integrate such techniques into a user's everyday viewing and to remain natural, however, would be a significant challenge. Open APIs for online streaming services, such as YouTube, afford integration to applications with relative ease, but offering integration into a variety of viewing contexts, including users' cable/terrestrial TV viewing is a significant challenge. Perhaps, as viewing habits converge to on-demand, online video, this will be more realisable.

More broadly, much of this research can be furthered with the addition of simple sensing technologies to aid us in further understanding user needs by inferring their interruptibility, mood and current activities to provide content and data which 
is appropriate. Work on indicative usage models, such as that by Leroy et al. [85], seeks to look at how we may infer television watchers' interest using ${ }_{3} \mathrm{D}$ head pose estimation aims to understand what types of content content users are interested in and therefore adjust their viewer profile, allowing more bespoke content to be provided. Further, the IDInteraction Project ${ }^{2}$, aims to inform devices through inferences about data captured what a user may want, in terms of content, at a given moment.

Such research may extend our research by learning when a given feature may be required - by capturing how the user is behaving one may infer needs and therefore provide appropriate interventions and content. In the context of our work, taking mediating attention as an example; with inertial sensor data, a device can infer that a user has put a device down to rest on their sofa, and therefore provide appropriate level of notification to make the user aware that new content has arrived. Finally, this thesis does not explore a combination of interventions. For instance, future research could consider the use of multiple interventions towards a common goal - a mixture of the manipulation of attention methods described in chapters 6 and 7 could allow a designer to drive attention towards a device with the intention of presenting more or less complex content for a desired outcome. For instance, in times of less attention-focused moments of a programme, one could initiate attention shifts towards more information-rich content on a mobile device.

Beyond the scope of this thesis, many of our findings and techniques may be broadly applicable to more than the multi-device media use case. Multi-device ecosystems are ubiquitous, and many may be supported by elements of this thesis, especially as second screening is one of the most ubiquitous cross-device scenarios 
and a potential exemplar for interaction designers. For example, the concepts and research methodology explored when investigating mediating attention in Chapter 7 may assist us in better enabling control, and prioritisation of, attention in complex multi-modal multi-screen environments, such as aircraft cockpits.

Concepts such as display commonalities may be applied in any scenario where a user is tasked with monitoring a series of events - by strategically employing unattended feeds of information within attended streams, one may reduce the cognitive demands and attention switching costs associated with many cross-device and multi-display environments. Further, the implications for the way we affect users' attention may have implications for the teaching scenarios described in the background of this thesis (Chapter 2). For instance, methodologies of curating the complexity of educational tablet content in the classroom dependent on how much the instructor is engaging with the students, may promote more managed active and passive learning.

The work in this thesis began in early 2014, when uptake of mobile devices in the living room was a rapidly increasing phenomenon. Since then, the dreams of the connected living room and streaming services have become mostly realised for the citizens of developed countries. Indeed, over the course of the past 3 years the average living room landscape has changed irreversibly. One could argue that, as the mobile device has become more prominent in our lives, the television has become the 'second screen'. 
This considered, with its superior viewing characteristics and affordances for shared experiences with co-located individuals, it will likely not become obsolete, but a skeuomorph of its former self. Now, with the multitude of internet enabled connected screens in our home, and the increasing ambiguity of 'television', it is vital that future media is driven not only by creative flair, but also shaped by rigorous human-factors research. 



\section{Appendix A}

\section{Online Questionnaire}




\section{Overview of Questionnaire}

The following questions are designed to assess your current experience with dualscreen television. If you conduct this questionnaire, you should to some extent engage in second screening. By second screen we refer to any handheld device you interact with while watching TV, for example a laptop, smartphone, or tablet.

The questions will first focus on your demographics - age, viewing habits, etc. - then probe, more specifically, your experience when second screening. Some questions are mandatory, some are not. If you are not sure, or do not want to answer a question, place 'N/A' in the answer field.

For the experiment you will have the chance to be awarded with 1 of 3 prizes one $£ 50$ and two $£ 25$. Participants will be randomly chosen.

Before undertaking the experiment please read the consent form and bill of rights forms thoroughly: http://cs.swan.ac.uk/ cstneate/docs/

By continuing with this experiment, you indicate that you agree with its terms and conditions.

${ }^{*}$ Required

\section{Demographics: About You}

1. Please state your age. *

2. Please state your gender*

3. Is English your first language? *

Tick all that apply.

Yes

No

4. If you answered 'no' to the previous question, what is your first language?

5. How many hours/minutes (can be less than $1 \mathrm{hr}$ ) on average of video media do you watch per day? This includes catch up services and streaming websites such as YouTube. *

6 . What type of shows do you generally watch? If you responded ' 0 ' for the previous question leave the answer blank. * 
7. Do you ever search for additional content related to the show you are watching on a secondary device, such as a tablet computer or smartphone? *

Tick all that apply.

Yes

No

8. Concerning the previous question. If you said yes, please give an example, and note the device(s) that you use to do so. *

9. Are you often involved with other activities while watching television (e.g. talking to family, or social networking)? If so, please list the main activities. *

10. I often use touch screen devices such as smartphones or tablet computers. *

Mark only one oval.

Strongly disagree

11. I find that I am easily distracted by content on my devices (tablet, smartphone, etc.), such as social media, when watching television. *

Mark only one oval.

Strongly disagree $\square \longrightarrow C$ Strongly agree

12. I easily get absorbed in television and lose all sense of time. *

Mark only one oval.

Strongly disagree

13. I often have the television on in the background while doing other things. * Mark only one oval.

Strongly agree




\section{Questions}

We will now ask about your second screening experience. Please read all questions carefully.

14. When I watch TV I experience: *

Mark only one oval.

\begin{tabular}{lllllll}
1 & 2 & 3 & 4 & 5 & \\
Low mental effort & $\square$ & & \\
\hline
\end{tabular}

15. When I watch TV while using a secondary device (i.e. a smartphone) I experience: * Mark only one oval.

$\begin{array}{lllllll}1 & 2 & 3 & 4 & 5 & \\ \text { Low mental effort } & \square & \square & \end{array}$

16. When I watch TV while searching for related information (like Googling an actor) this requires: *

Mark only one oval.

$\begin{array}{llllll}1 & 2 & 3 & 4 & 5 & \\ \text { Low mental effort } \square & \square & \end{array}$

17. When I watch TV while social networking (like using Facebook) on a second screen this requires: *

Mark only one oval.

$\begin{array}{llllll}1 & 2 & 3 & 4 & 5 & \\ \text { Low mental effort } & \square & \longrightarrow & \end{array}$

18. When I watch TV while engaged with a second screen I am aware of visual content happening on the TV (e.g. if a scene changes). *

Mark only one oval.

$\begin{array}{llllll}1 & 2 & 3 & 4 & 5 & \\ \text { Strongly disagree } \square & \square & \end{array}$

19. When I watch TV and view a secondary screen I am aware of auditory content on the TV (e.g. what people say). *

Mark only one oval.

$\begin{array}{lllllll}1 & 2 & 3 & 4 & 5 & \\ \text { Strongly disagree } \square & \square & \end{array}$


20. When I watch TV and view a secondary screen I am aware of what's happening visually on my secondary device (e.g. social network feeds updating). *

Mark only one oval.

Strongly disagree

21. Certain types of programmes require more mental effort to watch while second screening:

Mark only one oval.

$\begin{array}{lllll}1 & 2 & 3 & 4\end{array}$

22. If you agreed to some extent with the previous question, can you note some genres/shows that require more effort.

23. I am likely to focus on the second screen content when I accompany my TV viewing with a second screen.

Mark only one oval.

Strongly disagree

24. I am likely to focus on the TV programme when I accompany my TV viewing with a second screen.

Mark only one oval.

Strongly disagree

25. I am more likely to use a second screen for some shows than others. * Mark only one oval.

Strongly disagree


26. Regarding the previous question please note some shows/genres you're more likely to second screen for. Feel free to elaborate why. *

\section{End of Questionnaire}

The questionnaire is now done, and you get to enter the prize draw!

27. Please enter your email address below if you wish to enter the prize draw. The winners will be randomly chosen, and your email addresses will only be used to inform the prizewinners. 


\section{Appendix B}

\section{Visual Complexity Questionnaire}




\title{
Second Screen Visual Complexity
}

\author{
Participant Data Form
}

Participant Number:

Please state your gender.

Please state your age.

How many hours (or minutes) on average of video media do you watch per day? This includes catchup services and streaming websites such as YouTube.

What type of shows do you generally watch? If you responded '0' for the previous question leave the answer blank.

Do you ever search for additional content related to the show you are watching on a secondary device, such as a tablet computer or smartphone? If so, please provide an example. Noting the device(s) that you use to do so.

$\square \quad$ Yes -

$\square \quad$ No

Are you often involved with other activities while watching television (e.g. talking to family, or social networking)? If so, please list the main activities.

$\square \quad$ Yes

$\square \quad$ No

Do you have any uncorrected visual or hearing impairments? If so, please elaborate.

$\square \quad$ Yes -

$\square \quad$ No 
State how much you agree with the following statements:

I often use touch screen devices such as smartphones or tablet computers.

$\begin{array}{lccccc} & \text { Strongly } & \text { Agree } & \text { Neutral } & \text { Disagree } & \text { Strongly } \\ & \text { Agree } & & & \text { Disagree } \\ \text { Rating: } & \square & \square & \square & \square & \square\end{array}$

Rating:

I find that I am easily distracted by content on my devices (tablet, smartphone, etc), such as social media, when watching television.

\begin{tabular}{|c|c|c|c|c|c|}
\hline & $\begin{array}{c}\text { Strongly } \\
\text { Agree }\end{array}$ & Agree & Neutral & Disagree & $\begin{array}{l}\text { Strongly } \\
\text { Disagree }\end{array}$ \\
\hline ating: & $\square$ & $\square$ & $\square$ & $\square$ & $\square$ \\
\hline
\end{tabular}

I easily get absorbed in television and lose all sense of time.

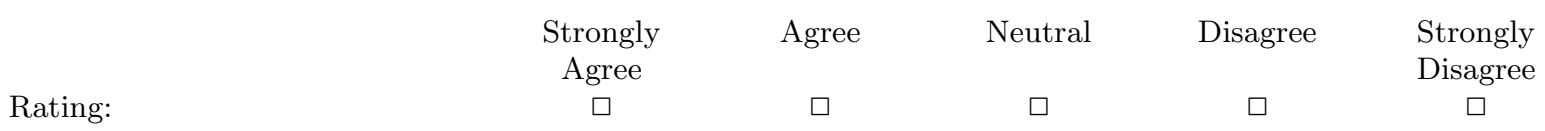

I often have the television on in the background while doing other things.

\begin{tabular}{|c|c|c|c|c|c|}
\hline & $\begin{array}{l}\text { Strongly } \\
\text { Agree }\end{array}$ & Agree & Neutral & Disagree & $\begin{array}{l}\text { Strongly } \\
\text { Disagree }\end{array}$ \\
\hline ating: & $\square$ & $\square$ & $\square$ & $\square$ & $\square$ \\
\hline
\end{tabular}

This concludes your data collection, please contact the test administrator and we shall begin the experiment. 


\section{Clip 1 (Inca) Questionnaire}

Please answer the following questions about the content you just engaged with.

I found it challenging to take in the content on the television when second screen content appeared.

\begin{tabular}{|c|c|c|c|c|c|}
\hline & $\begin{array}{l}\text { Strongly } \\
\text { Agree }\end{array}$ & Agree & Neutral & Disagree & $\begin{array}{l}\text { Strongly } \\
\text { Disagree }\end{array}$ \\
\hline ating: & $\square$ & $\square$ & $\square$ & $\square$ & $\square$ \\
\hline
\end{tabular}

I found it challenging to take in the content on the tablet with the television distracting me.

$\begin{array}{lccccc} & \text { Strongly } & \text { Agree } & \text { Neutral } & \text { Disagree } & \text { Strongly } \\ & \text { Agree } & & & \text { Disagree } \\ \text { Rating: } & \square & \square & \square & \square & \square\end{array}$

I felt I was missing material on the $T V$ because of the tablet.

$\begin{array}{lccccc} & \text { Strongly } & \text { Agree } & \text { Neutral } & \text { Disagree } & \text { Strongly } \\ & \text { Agree } & & & \text { Disagree } \\ \text { Rating: } & \square & \square & \square & \square & \square\end{array}$

I felt I was missing material on the tablet because of the TV.

\begin{tabular}{|c|c|c|c|c|c|}
\hline & $\begin{array}{l}\text { Strongly } \\
\text { Agree }\end{array}$ & Agree & Neutral & Disagree & $\begin{array}{l}\text { Strongly } \\
\text { Disagree }\end{array}$ \\
\hline Rating: & $\square$ & $\square$ & $\square$ & $\square$ & $\square$ \\
\hline
\end{tabular}

I felt I could easily take in the picture-based content on the second screen content.

\begin{tabular}{|c|c|c|c|c|c|}
\hline & $\begin{array}{l}\text { Strongly } \\
\text { Agree }\end{array}$ & Agree & Neutral & Disagree & $\begin{array}{l}\text { Strongly } \\
\text { Disagree }\end{array}$ \\
\hline lating: & $\square$ & $\square$ & $\square$ & $\square$ & $\square$ \\
\hline
\end{tabular}

I felt I could easily take in the text-based content on the second screen content.

$\begin{array}{lccccc} & \text { Strongly } & \text { Agree } & \text { Neutral } & \text { Disagree } & \text { Strongly } \\ \text { Rating: } & \text { Agree } & & & \text { Disagree } \\ & \square & \square & \square & \square & \square\end{array}$

Rate, out of 10, how visually complex you found the content on the second screen. 


\section{Clip 2 (Snooker) Questionnaire}

Please answer the following questions about the content you just engaged with.

I found it challenging to take in the content on the television when second screen content appeared.

\begin{tabular}{|c|c|c|c|c|c|}
\hline & $\begin{array}{l}\text { Strongly } \\
\text { Agree }\end{array}$ & Agree & Neutral & Disagree & $\begin{array}{l}\text { Strongly } \\
\text { Disagree }\end{array}$ \\
\hline ating: & $\square$ & $\square$ & $\square$ & $\square$ & $\square$ \\
\hline
\end{tabular}

I found it challenging to take in the content on the tablet with the television distracting me.

$\begin{array}{lccccc} & \text { Strongly } & \text { Agree } & \text { Neutral } & \text { Disagree } & \text { Strongly } \\ & \text { Agree } & & & \text { Disagree } \\ \text { Rating: } & \square & \square & \square & \square & \square\end{array}$

I felt I was missing material on the $T V$ because of the tablet.

\begin{tabular}{|c|c|c|c|c|c|}
\hline & $\begin{array}{l}\text { Strongly } \\
\text { Agree }\end{array}$ & Agree & Neutral & Disagree & $\begin{array}{l}\text { Strongly } \\
\text { Disagree }\end{array}$ \\
\hline ating: & $\square$ & $\square$ & $\square$ & $\square$ & $\square$ \\
\hline
\end{tabular}

I felt I was missing material on the tablet because of the TV.

\begin{tabular}{|c|c|c|c|c|c|}
\hline & $\begin{array}{l}\text { Strongly } \\
\text { Agree }\end{array}$ & Agree & Neutral & Disagree & $\begin{array}{l}\text { Strongly } \\
\text { Disagree }\end{array}$ \\
\hline lating: & $\square$ & $\square$ & $\square$ & $\square$ & $\square$ \\
\hline
\end{tabular}

I felt I could easily take in the picture-based content on the second screen content.

\begin{tabular}{|c|c|c|c|c|c|}
\hline & $\begin{array}{c}\text { Strongly } \\
\text { Agree }\end{array}$ & Agree & Neutral & Disagree & $\begin{array}{l}\text { Strongly } \\
\text { Disagree }\end{array}$ \\
\hline lating: & $\square$ & $\square$ & $\square$ & $\square$ & $\square$ \\
\hline
\end{tabular}

I felt I could easily take in the text-based content on the second screen content.

$\begin{array}{lccccc} & \text { Strongly } & \text { Agree } & \text { Neutral } & \text { Disagree } & \text { Strongly } \\ & \text { Agree } & & & \text { Disagree } \\ \text { Rating: } & \square & \square & \square & \square & \square\end{array}$

Rate, out of 10, how visually complex you found the content on the second screen. 


\section{Clip 3 (Click) Questionnaire}

Please answer the following questions about the content you just engaged with.

I found it challenging to take in the content on the television when second screen content appeared.

\begin{tabular}{|c|c|c|c|c|c|}
\hline & $\begin{array}{l}\text { Strongly } \\
\text { Agree }\end{array}$ & Agree & Neutral & Disagree & $\begin{array}{l}\text { Strongly } \\
\text { Disagree }\end{array}$ \\
\hline ating: & $\square$ & $\square$ & $\square$ & $\square$ & $\square$ \\
\hline
\end{tabular}

I found it challenging to take in the content on the tablet with the television distracting me.

$\begin{array}{lccccc} & \text { Strongly } & \text { Agree } & \text { Neutral } & \text { Disagree } & \text { Strongly } \\ & \text { Agree } & & & \text { Disagree } \\ \text { Rating: } & \square & \square & \square & \square & \square\end{array}$

I felt I was missing material on the $T V$ because of the tablet.

$\begin{array}{lccccc} & \text { Strongly } & \text { Agree } & \text { Neutral } & \text { Disagree } & \text { Strongly } \\ & \text { Agree } & & & \text { Disagree } \\ \text { Rating: } & \square & \square & \square & \square & \square\end{array}$

I felt I was missing material on the tablet because of the TV.

\begin{tabular}{|c|c|c|c|c|c|}
\hline & $\begin{array}{l}\text { Strongly } \\
\text { Agree }\end{array}$ & Agree & Neutral & Disagree & $\begin{array}{l}\text { Strongly } \\
\text { Disagree }\end{array}$ \\
\hline Rating: & $\square$ & $\square$ & $\square$ & $\square$ & $\square$ \\
\hline
\end{tabular}

I felt I could easily take in the picture-based content on the second screen content.

$\begin{array}{lccccc} & \text { Strongly } & \text { Agree } & \text { Neutral } & \text { Disagree } & \text { Strongly } \\ & \text { Agree } & & & \text { Disagree } \\ \text { Rating: } & \square & \square & \square & \square & \square\end{array}$

I felt I could easily take in the text-based content on the second screen content.

$\begin{array}{lccccc} & \text { Strongly } & \text { Agree } & \text { Neutral } & \text { Disagree } & \text { Strongly } \\ & \text { Agree } & & & \text { Disagree } \\ \text { Rating: } & \square & \square & \square & \square & \square\end{array}$

Rate, out of 10 , how visually complex you found the content on the second screen. 


\section{Clip 4 (Eggheads) Questionnaire}

Please answer the following questions about the content you just engaged with.

I found it challenging to take in the content on the television when second screen content appeared.

\begin{tabular}{|c|c|c|c|c|c|}
\hline & $\begin{array}{l}\text { Strongly } \\
\text { Agree }\end{array}$ & Agree & Neutral & Disagree & $\begin{array}{l}\text { Strongly } \\
\text { Disagree }\end{array}$ \\
\hline ating: & $\square$ & $\square$ & $\square$ & $\square$ & $\square$ \\
\hline
\end{tabular}

I found it challenging to take in the content on the tablet with the television distracting me.

$\begin{array}{lccccc} & \text { Strongly } & \text { Agree } & \text { Neutral } & \text { Disagree } & \text { Strongly } \\ \text { Rating: } & \text { Agree } & & & \text { Disagree } \\ \text { Ren } & \square & \square & \square & \square & \square\end{array}$

I felt I was missing material on the $T V$ because of the tablet.

$\begin{array}{lccccc} & \text { Strongly } & \text { Agree } & \text { Neutral } & \text { Disagree } & \text { Strongly } \\ & \text { Agree } & & & \text { Disagree } \\ \text { Rating: } & \square & \square & \square & \square & \square\end{array}$

I felt I was missing material on the tablet because of the TV.

\begin{tabular}{|c|c|c|c|c|c|}
\hline & $\begin{array}{l}\text { Strongly } \\
\text { Agree }\end{array}$ & Agree & Neutral & Disagree & $\begin{array}{l}\text { Strongly } \\
\text { Disagree }\end{array}$ \\
\hline Rating: & $\square$ & $\square$ & $\square$ & $\square$ & $\square$ \\
\hline
\end{tabular}

I felt I could easily take in the picture-based content on the second screen content.

\begin{tabular}{|c|c|c|c|c|c|}
\hline & $\begin{array}{l}\text { Strongly } \\
\text { Agree }\end{array}$ & Agree & Neutral & Disagree & $\begin{array}{l}\text { Strongly } \\
\text { Disagree }\end{array}$ \\
\hline lating: & $\square$ & $\square$ & $\square$ & $\square$ & $\square$ \\
\hline
\end{tabular}

I felt I could easily take in the text-based content on the second screen content.

$\begin{array}{lccccc} & \text { Strongly } & \text { Agree } & \text { Neutral } & \text { Disagree } & \text { Strongly } \\ & \text { Agree } & & & \text { Disagree } \\ \text { Rating: } & \square & \square & \square & \square & \square\end{array}$

Rate, out of 10 , how visually complex you found the content on the second screen. 


\section{Post Study Questionnaire}

Please answer this short questionnaire so that we may gather your impressions of the second screen content.

The more detailed information on the second screen positively impacted my experience.

$\begin{array}{lccccc} & \text { Strongly } & \text { Agree } & \text { Neutral } & \text { Disagree } & \text { Strongly } \\ & \text { Agree } & & & \text { Disagree } \\ \text { Rating: } & \square & \square & \square & \square & \square\end{array}$

The less detailed information on the second screen positively impacted my experience.

\begin{tabular}{|c|c|c|c|c|c|}
\hline & $\begin{array}{c}\text { Strongly } \\
\text { Agree }\end{array}$ & Agree & Neutral & Disagree & $\begin{array}{l}\text { Strongly } \\
\text { Disagree }\end{array}$ \\
\hline ating: & $\square$ & $\square$ & $\square$ & $\square$ & $\square$ \\
\hline
\end{tabular}

Sometimes I found it challenging to take in all the material on both screens at the same time.

\begin{tabular}{|c|c|c|c|c|c|}
\hline & $\begin{array}{l}\text { Strongly } \\
\text { Agree }\end{array}$ & Agree & Neutral & Disagree & $\begin{array}{l}\text { Strongly } \\
\text { Disagree }\end{array}$ \\
\hline ating: & $\square$ & $\square$ & $\square$ & $\square$ & $\square$ \\
\hline
\end{tabular}

I found it easy to focus on both the second screen content, and the television at all times.

\begin{tabular}{|c|c|c|c|c|c|}
\hline & $\begin{array}{c}\text { Strongly } \\
\text { Agree }\end{array}$ & Agree & Neutral & Disagree & $\begin{array}{l}\text { Strongly } \\
\text { Disagree }\end{array}$ \\
\hline ating: & $\square$ & $\square$ & $\square$ & $\square$ & $\square$ \\
\hline
\end{tabular}




\section{Appendix C}

Complexity Adjustment

Questionnaire 


\title{
Second Screen Complexity Adjustment Experiment
}

\author{
Participant Data Form
}

Participant Number:

Please state your gender.

Please state your age.

How many hours (or minutes) on average of video media do you watch per day? This includes catchup services and streaming websites such as YouTube.

What type of shows do you generally watch? If you responded '0' for the previous question leave the answer blank.

Do you ever search for additional content related to the show you are watching on a secondary device, such as a tablet computer or smartphone? If so, please provide an example. Noting the device(s) that you use to do so.

$\square \quad$ Yes -

$\square \quad$ No

Are you often involved with other activities while watching television (e.g. talking to family, or social networking)? If so, please list the main activities.

$\square \quad$ Yes -

$\square \quad$ No

Have you ever used a 'companion application' - an app designed to go along with a TV show or series?

$\square \quad$ Yes -

$\square \quad$ No

Do you have any uncorrected visual or hearing impairments? If so, please elaborate.

$\square \quad$ Yes -

$\square \quad$ No 
State how much you agree with the following statements

I often use touch screen devices such as smartphones or tablet computers.

$\begin{array}{lccccc} & \text { Strongly } & \text { Agree } & \text { Neutral } & \text { Disagree } & \text { Strongly } \\ & \text { Agree } & & & \text { Disagree } \\ \text { Rating: } & \square & \square & \square & \square & \square\end{array}$

I find that I am easily distracted by content on my devices (tablet, smartphone, etc), such as social media, when watching television.

$\begin{array}{lccccc} & \text { Strongly } & \text { Agree } & \text { Neutral } & \text { Disagree } & \text { Strongly } \\ & \text { Agree } & & & \text { Disagree } \\ \text { Rating: } & \square & \square & \square & \square & \square\end{array}$

I easily get absorbed in television and lose all sense of time.

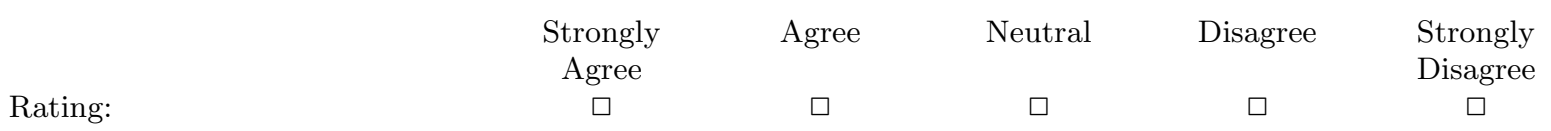

I often have the television on in the background while doing other things.

\begin{tabular}{|c|c|c|c|c|c|}
\hline & $\begin{array}{l}\text { Strongly } \\
\text { Agree }\end{array}$ & Agree & Neutral & Disagree & $\begin{array}{l}\text { Strongly } \\
\text { Disagree }\end{array}$ \\
\hline lating: & $\square$ & $\square$ & $\square$ & $\square$ & $\square$ \\
\hline
\end{tabular}

I often miss something on the TV while engaging with a secondary device (like a smartphone, tablet, laptop).

\begin{tabular}{|c|c|c|c|c|c|}
\hline & $\begin{array}{l}\text { Strongly } \\
\text { Agree }\end{array}$ & Agree & Neutral & Disagree & $\begin{array}{l}\text { Strongly } \\
\text { Disagree }\end{array}$ \\
\hline Rating: & $\square$ & $\square$ & $\square$ & $\square$ & $\square$ \\
\hline
\end{tabular}

This concludes your data collection, please contact the test administrator and we shall begin the experiment. 


\section{Clip 1 - Africa: Sahara}

Please answer the following questions about the content you just engaged with.

I found the level of detail on the tablet allowed me to take in the content on the tablet.

$\begin{array}{lccccc} & \text { Strongly } & \text { Agree } & \text { Neutral } & \text { Disagree } & \text { Strongly } \\ & \text { Agree } & & & \text { Disagree } \\ \text { Rating: } & \square & \square & \square & \square & \square\end{array}$

I found the level of detail on the tablet allowed me to take in the content on the television.

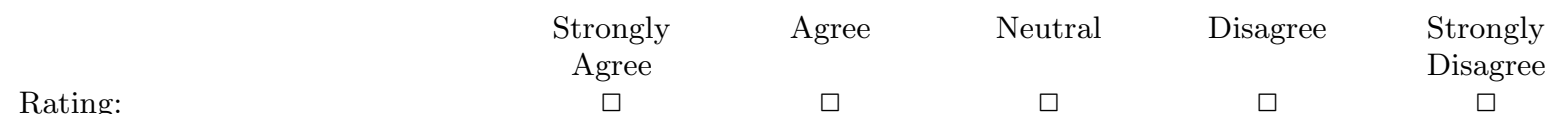

I found that the level of detail on the tablet allowed me to pay attention to both the TV programme, and the tablet content.

$\begin{array}{lccccc} & \text { Strongly } & \text { Agree } & \text { Neutral } & \text { Disagree } & \text { Strongly } \\ & \text { Agree } & & & \text { Disagree } \\ \text { Rating: } & \square & \square & \square & \square & \square\end{array}$

The attention required to watch the TV meant that I had to sacrifice viewing a display to engage with the other.

$\begin{array}{lccccc} & \text { Strongly } & \text { Agree } & \text { Neutral } & \text { Disagree } & \text { Strongly } \\ & \text { Agree } & & & \text { Disagree } \\ \text { Rating: } & \square & \square & \square & \square & \square\end{array}$

The level of detail on the tablet meant that I had to sacrifice viewing a display to engage with the other.

\begin{tabular}{|c|c|c|c|c|c|}
\hline & $\begin{array}{c}\text { Strongly } \\
\text { Agree }\end{array}$ & Agree & Neutral & Disagree & $\begin{array}{l}\text { Strongly } \\
\text { Disagree }\end{array}$ \\
\hline lating: & $\square$ & $\square$ & $\square$ & $\square$ & $\square$ \\
\hline
\end{tabular}

I felt the level of detail on the tablet was...

Rating:

Too little.

Just right.

Too much.

$\square$

$\square$

$\square$ 


\section{Clip 2 - The Weakest Link}

Please answer the following questions about the content you just engaged with.

I found the level of detail on the tablet allowed me to take in the content on the tablet.

$\begin{array}{lccccc} & \text { Strongly } & \text { Agree } & \text { Neutral } & \text { Disagree } & \text { Strongly } \\ & \text { Agree } & & & \text { Disagree } \\ \text { Rating: } & \square & \square & \square & \square & \square\end{array}$

I found the level of detail on the tablet allowed me to take in the content on the television.

$\begin{array}{lccccc} & \text { Strongly } & \text { Agree } & \text { Neutral } & \text { Disagree } & \text { Strongly } \\ & \text { Agree } & & & \text { Disagree } \\ \text { Rating: } & \square & \square & \square & \square & \square\end{array}$

I found that the level of detail on the tablet allowed me to pay attention to both the TV programme, and the tablet content.

\begin{tabular}{|c|c|c|c|c|c|}
\hline & $\begin{array}{c}\text { Strongly } \\
\text { Agree }\end{array}$ & Agree & Neutral & Disagree & $\begin{array}{l}\text { Strongly } \\
\text { Disagree }\end{array}$ \\
\hline Rating: & $\square$ & $\square$ & $\square$ & $\square$ & $\square$ \\
\hline
\end{tabular}

The attention required to watch the TV meant that I had to sacrifice viewing a display to engage with the other.

\begin{tabular}{|c|c|c|c|c|c|}
\hline & $\begin{array}{l}\text { Strongly } \\
\text { Agree }\end{array}$ & Agree & Neutral & Disagree & $\begin{array}{l}\text { Strongly } \\
\text { Disagree }\end{array}$ \\
\hline Rating: & $\square$ & $\square$ & $\square$ & $\square$ & $\square$ \\
\hline
\end{tabular}

The level of detail on the tablet meant that I had to sacrifice viewing a display to engage with the other.

\begin{tabular}{|c|c|c|c|c|c|}
\hline & $\begin{array}{l}\text { Strongly } \\
\text { Agree }\end{array}$ & Agree & Neutral & Disagree & $\begin{array}{l}\text { Strongly } \\
\text { Disagree }\end{array}$ \\
\hline Rating: & $\square$ & $\square$ & $\square$ & $\square$ & $\square$ \\
\hline
\end{tabular}

I felt the level of detail on the tablet was...

Rating:

Too little.

Just right.

Too much.

$\square$

$\square$

$\square$ 


\section{Clip 3 - James Martin: Home Comforts}

Please answer the following questions about the content you just engaged with.

I found the level of detail on the tablet allowed me to take in the content on the tablet.

$\begin{array}{lccccc} & \text { Strongly } & \text { Agree } & \text { Neutral } & \text { Disagree } & \text { Strongly } \\ & \text { Agree } & & & \text { Disagree } \\ \text { Rating: } & \square & \square & \square & \square & \square\end{array}$

I found the level of detail on the tablet allowed me to take in the content on the television.

$\begin{array}{lccccc} & \text { Strongly } & \text { Agree } & \text { Neutral } & \text { Disagree } & \text { Strongly } \\ & \text { Agree } & & & \text { Disagree } \\ \text { Rating: } & \square & \square & \square & \square & \square\end{array}$

I found that the level of detail on the tablet allowed me to pay attention to both the TV programme, and the tablet content.

\begin{tabular}{|c|c|c|c|c|c|}
\hline & $\begin{array}{l}\text { Strongly } \\
\text { Agree }\end{array}$ & Agree & Neutral & Disagree & $\begin{array}{l}\text { Strongly } \\
\text { Disagree }\end{array}$ \\
\hline Rating: & $\square$ & $\square$ & $\square$ & $\square$ & $\square$ \\
\hline
\end{tabular}

The attention required to watch the TV meant that I had to sacrifice viewing a display to engage with the other.

\begin{tabular}{|c|c|c|c|c|c|}
\hline & $\begin{array}{l}\text { Strongly } \\
\text { Agree }\end{array}$ & Agree & Neutral & Disagree & $\begin{array}{l}\text { Strongly } \\
\text { Disagree }\end{array}$ \\
\hline Rating: & $\square$ & $\square$ & $\square$ & $\square$ & $\square$ \\
\hline
\end{tabular}

The level of detail on the tablet meant that I had to sacrifice viewing a display to engage with the other.

\begin{tabular}{|c|c|c|c|c|c|}
\hline & $\begin{array}{c}\text { Strongly } \\
\text { Agree }\end{array}$ & Agree & Neutral & Disagree & $\begin{array}{l}\text { Strongly } \\
\text { Disagree }\end{array}$ \\
\hline lating: & $\square$ & $\square$ & $\square$ & $\square$ & $\square$ \\
\hline
\end{tabular}

I felt the level of detail on the tablet was...

Rating:

Too little.

Just right.

Too much.

$\square$

$\square$

$\square$ 


\section{Post Study Questionnaire}

Please answer this short questionnaire so that we may gather your impressions of the second screen content.

I felt that the detail of tablet content in the first clip (Sahara) enhanced the dual screen experience as a whole

$\begin{array}{lccccc} & \text { Strongly } & \text { Agree } & \text { Neutral } & \text { Disagree } & \text { Strongly } \\ & \text { Agree } & & & \text { Disagree } \\ \text { Rating: } & \square & \square & \square & \square & \square\end{array}$

I felt that the detail of tablet content in the second clip (The Weakest Link) enhanced the dual screen experience as a whole

$\begin{array}{lccccc} & \text { Strongly } & \text { Agree } & \text { Neutral } & \text { Disagree } & \text { Strongly } \\ & \text { Agree } & & & \text { Disagree } \\ \text { Rating: } & \square & \square & \square & \square & \square\end{array}$

I felt that the detail of tablet content in the third clip (Home Comforts) enhanced the dual screen experience as a whole

\begin{tabular}{|c|c|c|c|c|c|}
\hline & $\begin{array}{c}\text { Strongly } \\
\text { Agree }\end{array}$ & Agree & Neutral & Disagree & $\begin{array}{l}\text { Strongly } \\
\text { Disagree }\end{array}$ \\
\hline Rating: & $\square$ & $\square$ & $\square$ & $\square$ & $\square$ \\
\hline
\end{tabular}

I enjoy watching documentaries like Sahara.

$\begin{array}{cccccc} & \text { Strongly } & \text { Agree } & \text { Neutral } & \text { Disagree } & \text { Strongly } \\ & \text { Agree } & & & \text { Disagree } \\ \text { Rating: } & \square & \square & \square & \square & \square\end{array}$

I enjoy watching quiz shows such as The Weakest Link.

\begin{tabular}{|c|c|c|c|c|c|}
\hline & $\begin{array}{c}\text { Strongly } \\
\text { Agree }\end{array}$ & Agree & Neutral & Disagree & $\begin{array}{l}\text { Strongly } \\
\text { Disagree }\end{array}$ \\
\hline Rating: & $\square$ & $\square$ & $\square$ & $\square$ & $\square$ \\
\hline
\end{tabular}

I enjoy watching shows about cooking like Home Comforts.

\begin{tabular}{|c|c|c|c|c|c|}
\hline & $\begin{array}{l}\text { Strongly } \\
\text { Agree }\end{array}$ & Agree & Neutral & Disagree & $\begin{array}{l}\text { Strongly } \\
\text { Disagree }\end{array}$ \\
\hline ating: & $\square$ & $\square$ & $\square$ & $\square$ & $\square$ \\
\hline
\end{tabular}





\section{Appendix D}

Mediating Attention Questionnaire 


\title{
Designing Alerts for Media Contexts
}

\author{
Participant Data
}

Participant Number:

Please state your gender.

Please state your age.

How many hours on average of media do you watch per day? This includes catchup services and streaming websites such as YouTube.

What type of shows do you generally watch? If you responded ' 0 ' for the previous question leave the answer blank.

On a scale of 1 to 10 rate how much you like nature documentaries.

Do you ever search for additional content related to the show on a secondary device, such as a tablet computer or smartphone? If so, please provide an example. Noting the device(s) that you use to do so.

Yes -

$\square \quad$ No 
Do you have any uncorrected visual or hearing impairments? If so, please elaborate.

$$
\text { Yes - }
$$

No

\section{State how much you agree with the following statements:}

I often use touch screen devices such as smartphones or tablet computers.

\begin{tabular}{|c|c|c|c|c|}
\hline & $\begin{array}{c}\text { Strongly } \\
\text { Agree }\end{array}$ & Agree & Neutral & Disagree \\
\hline ating. & $\square$ & $\square$ & $\square$ & $\square$ \\
\hline
\end{tabular}

I find that I am easily distracted by content on my devices (tablet, smartphone, etc), such as social media, when watching television.

\begin{tabular}{|c|c|c|c|c|}
\hline & $\begin{array}{c}\text { Strongly } \\
\text { Agree }\end{array}$ & Agree & Neutral & Disagree \\
\hline Rating: & $\square$ & $\square$ & $\square$ & $\square$ \\
\hline
\end{tabular}

I easily get absorbed in television and lose all sense of time.

$\begin{array}{cccc}\text { Strongly Agree Neutral Disagree } & \text { Strongly } \\ \text { Agree } & \text { Disagree }\end{array}$

Rating:

This concludes your data collection. Please contact the test administrator so that we may begin the experiment. 


\section{Post-test questionnaire}

This section is designed to get your subjective feedback about the techniques used to alert you to the new content while you were watching television.

Please answer the following questions about the noticeability of the alerting techniques. If you did not notice a specific type of alert, note below.

I found that when the content appeared with no alert I was drawn to the new content on the iPad.

$\begin{array}{cccc}\begin{array}{c}\text { Strongly Agree Neutral Disagree } \\ \text { Agree }\end{array} & \text { Strongly } \\ \text { Disagree }\end{array}$

Rating:

I found that when the content appeared with animation (shaking) I was drawn to the new content on the iPad.

\begin{tabular}{|c|c|c|c|c|}
\hline & $\begin{array}{c}\text { Strongly } \\
\text { Agree }\end{array}$ & Agree & Neutral & Disagree \\
\hline Rating: & $\square$ & $\square$ & $\square$ & $\square$ \\
\hline
\end{tabular}

I found that when the content appeared with musical sounds I was drawn to the new content on the iPad.

\begin{tabular}{|c|c|c|c|c|c|}
\hline & $\begin{array}{c}\text { Strongly } \\
\text { Agree }\end{array}$ & Agree & Neutral & Disagree & $\begin{array}{l}\text { Strongly } \\
\text { Disagree }\end{array}$ \\
\hline Rating: & $\square$ & $\square$ & $\square$ & $\square$ & $\square$ \\
\hline
\end{tabular}

I found that when the content appeared with related sounds I was drawn to the new content on the iPad.

\begin{tabular}{|c|c|c|c|c|c|}
\hline & $\begin{array}{c}\text { Strongly } \\
\text { Agree }\end{array}$ & Agree & Neutral & Disagree & $\begin{array}{l}\text { Strongly } \\
\text { Disagree }\end{array}$ \\
\hline Rating: & $\square$ & $\square$ & $\square$ & $\square$ & $\square$ \\
\hline
\end{tabular}


I found that when the content appeared on the television with no animation I was drawn to the new content on the iPad.

$\begin{array}{llll}\text { Strongly Agree Neutral Disagree } & \text { Strongly } \\ \text { Agree } & & \text { Disagree }\end{array}$

Rating:

I found that when the content appeared on the television with animation (shaking) I was drawn to the new content on the iPad.

\begin{tabular}{|c|c|c|c|c|c|}
\hline & $\begin{array}{c}\text { Strongly } \\
\text { Agree }\end{array}$ & Agree & Neutral & Disagree & $\begin{array}{l}\text { Strongly } \\
\text { Disagree }\end{array}$ \\
\hline ating: & $\square$ & $\square$ & $\square$ & $\square$ & $\square$ \\
\hline
\end{tabular}

Please answer the following questions about your experience of switching between the two screens. If you did not notice a specific type of alert, note below.

I found that when the content appeared on the device with no alert the transition to the second screen was natural.

\begin{tabular}{|c|c|c|c|c|c|}
\hline & $\begin{array}{c}\text { Strongly } \\
\text { Agree }\end{array}$ & Agree & Neutral & Disagree & $\begin{array}{l}\text { Strongly } \\
\text { Disagree }\end{array}$ \\
\hline Rating: & $\square$ & $\square$ & $\square$ & $\square$ & $\square$ \\
\hline
\end{tabular}

I found that when the content appeared on the device with animation (shaking) the transition to the second screen was natural.

\begin{tabular}{|c|c|c|c|c|c|}
\hline & $\begin{array}{c}\text { Strongly } \\
\text { Agree }\end{array}$ & Agree & Neutral & Disagree & $\begin{array}{l}\text { Strongly } \\
\text { Disagree }\end{array}$ \\
\hline Rating: & $\square$ & $\square$ & $\square$ & $\square$ & $\square$ \\
\hline
\end{tabular}

I found that when the content appeared with a musical sound the transition to the second screen was natural.

Rating:

\begin{tabular}{|c|c|c|c|}
\hline $\begin{array}{c}\text { Strongly } \\
\text { Agree }\end{array}$ & Agre & Neutral & Disagree \\
\hline
\end{tabular}


I found that when the content appeared with a related sound the transition to the second screen was natural.

$\begin{array}{ccccc}\text { Strongly Agree Neutral Disagree } & \text { Strongly } \\ \text { Agree } & & \text { Disagree }\end{array}$

Rating:

I found that when the content appeared with the notification on the television the transition to the second screen was natural.

\begin{tabular}{|c|c|c|c|c|}
\hline & $\begin{array}{c}\text { Strongly } \\
\text { Agree }\end{array}$ & Agree & Neutral & Disagree \\
\hline Rating: & $\square$ & $\square$ & $\square$ & $\square$ \\
\hline
\end{tabular}

I found that when the content appeared with the animated (shaking) notification on the television the transition to the second screen was natural.

$\begin{array}{cccc}\begin{array}{c}\text { Strongly Agree Neutral Disagree } \\ \text { Agree }\end{array} & \begin{array}{c}\text { Strongly } \\ \text { Disagree }\end{array}\end{array}$

Rating:

Please rate the following alerting techniques with regards to your general preferences. Feel free to elaborate as to why on the line below.

I liked media appearing on device without an alert.

\begin{tabular}{|c|c|c|c|c|c|}
\hline & $\begin{array}{c}\text { Strongly } \\
\text { Agree }\end{array}$ & Agree & Neutral & Disagree & $\begin{array}{l}\text { Strongly } \\
\text { Disagree }\end{array}$ \\
\hline Rating: & $\square$ & $\square$ & $\square$ & $\square$ & $\square$ \\
\hline
\end{tabular}

Reason (if any): 
I liked media appearing on device with animation.

$\begin{array}{cccc}\text { Strongly Agree Neutral Disagree } & \text { Strongly } \\ \text { Agree } & & \text { Disagree }\end{array}$

Reason (if any):

I liked media appearing on device with a musical sound.

\begin{tabular}{|c|c|c|c|c|c|}
\hline & $\begin{array}{c}\text { Strongly } \\
\text { Agree }\end{array}$ & Agree & Neutral & Disagree & $\begin{array}{l}\text { Strongly } \\
\text { Disagree }\end{array}$ \\
\hline Rating: & $\square$ & $\square$ & $\square$ & $\square$ & $\square$ \\
\hline
\end{tabular}

Reason (if any):

I liked media appearing on device with a related sound.

\begin{tabular}{|c|c|c|c|c|c|}
\hline & $\begin{array}{c}\text { Strongly } \\
\text { Agree }\end{array}$ & Agree & Neutral & Disagree & $\begin{array}{l}\text { Strongly } \\
\text { Disagree }\end{array}$ \\
\hline ating: & $\square$ & $\square$ & $\square$ & $\square$ & $\square$ \\
\hline
\end{tabular}

Reason (if any):

I liked media appearing on the TV with no animation.

\begin{tabular}{|c|c|c|c|c|c|}
\hline & $\begin{array}{c}\text { Strongly } \\
\text { Agree }\end{array}$ & Agree & Neutral & Disagree & $\begin{array}{l}\text { Strongly } \\
\text { Disagree }\end{array}$ \\
\hline Rating: & $\square$ & $\square$ & $\square$ & $\square$ & $\square$ \\
\hline
\end{tabular}

Reason (if any): 
I liked media appearing on the TV with animation (shaking).

\begin{tabular}{|c|c|c|c|c|c|}
\hline & $\begin{array}{c}\text { Strongly } \\
\text { Agree }\end{array}$ & Agree & Neutral & Disagree & $\begin{array}{l}\text { Strongly } \\
\text { Disagree }\end{array}$ \\
\hline Rating: & $\square$ & $\square$ & $\square$ & $\square$ & $\square$ \\
\hline
\end{tabular}

Reason (if any):

\section{END OF EXPERIMENT}




\title{
Appendix E
}

\section{Display Commonalities}

\author{
Questionnaire
}




\section{Dual-screen Display Commonalities}

\section{Participant Data Form}

Participant Number:

Please state your gender.

Please state your age.

How many hours (or minutes) on average of video media do you watch per day? This includes catchup services and streaming websites such as YouTube.

What type of shows do you generally watch? If you responded ' 0 ' for the previous question leave the answer blank.

Do you ever search for additional content related to the show you are watching on a secondary device, such as a tablet computer or smartphone? If so, please provide an example. Noting the device(s) that you use to do so.

$\square \quad$ Yes -

$\square \quad$ No

Are you often involved with other activities while watching television (e.g. talking to family, or social networking)? If so, please list the main activities.

$\square \quad$ Yes -

$\square \quad$ No

Have you ever used a 'companion application' - an app designed to go along with a TV show or series?

$\square \quad$ Yes $-\ldots$

$\square \quad$ No

Do you have any uncorrected visual or hearing impairments? If so, please elaborate.

$\square \quad$ Yes - . . .

$\square \quad$ No 
State how much you agree with the following statements

I often use touch screen devices such as smartphones or tablet computers.

\begin{tabular}{|c|c|c|c|c|c|}
\hline & $\begin{array}{c}\text { Strongly } \\
\text { Agree }\end{array}$ & Agree & Neutral & Disagree & $\begin{array}{l}\text { Strongly } \\
\text { Disagree }\end{array}$ \\
\hline ating: & $\square$ & $\square$ & $\square$ & $\square$ & $\square$ \\
\hline
\end{tabular}

I find that I am easily distracted by content on my devices (tablet, smartphone, etc.), such as social media, when watching television.

\begin{tabular}{|c|c|c|c|c|c|}
\hline & $\begin{array}{c}\text { Strongly } \\
\text { Agree }\end{array}$ & Agree & Neutral & Disagree & $\begin{array}{l}\text { Strongly } \\
\text { Disagree }\end{array}$ \\
\hline Rating: & $\square$ & $\square$ & $\square$ & $\square$ & $\square$ \\
\hline
\end{tabular}

I easily get absorbed in television and lose all sense of time.

$\begin{array}{lccccc} & \text { Strongly } & \text { Agree } & \text { Neutral } & \text { Disagree } & \text { Strongly } \\ \text { Rating: } & \text { Agree } & \square & & & \text { Disagree } \\ \text { Ren } & \square & \square & \square & \square & \square\end{array}$

I often have the television on in the background while doing other things.

\begin{tabular}{|c|c|c|c|c|c|}
\hline & $\begin{array}{c}\text { Strongly } \\
\text { Agree }\end{array}$ & Agree & Neutral & Disagree & $\begin{array}{l}\text { Strongly } \\
\text { Disagree }\end{array}$ \\
\hline Rating: & $\square$ & $\square$ & $\square$ & $\square$ & $\square$ \\
\hline
\end{tabular}

I often miss something on the TV while engaging with a secondary device (like a smartphone, tablet, laptop).

\begin{tabular}{|c|c|c|c|c|c|}
\hline & $\begin{array}{l}\text { Strongly } \\
\text { Agree }\end{array}$ & Agree & Neutral & Disagree & $\begin{array}{l}\text { Strongly } \\
\text { Disagree }\end{array}$ \\
\hline Rating: & $\square$ & $\square$ & $\square$ & $\square$ & $\square$ \\
\hline
\end{tabular}

This concludes your initial data collection, please contact the test administrator and we shall begin the experiment. 


\section{Clip 1 - Wild China}

I found that I could take in the content over the two screens at the same time effectively.

\begin{tabular}{|c|c|c|c|c|c|}
\hline & $\begin{array}{c}\text { Strongly } \\
\text { Agree }\end{array}$ & Agree & Neutral & Disagree & $\begin{array}{l}\text { Strongly } \\
\text { Disagree }\end{array}$ \\
\hline Rating: & $\square$ & $\square$ & $\square$ & $\square$ & $\square$ \\
\hline
\end{tabular}

I had to shift my viewing between the screens a lot to take in the TV and tablet content.

\begin{tabular}{|c|c|c|c|c|c|}
\hline & $\begin{array}{c}\text { Strongly } \\
\text { Agree }\end{array}$ & Agree & Neutral & Disagree & $\begin{array}{l}\text { Strongly } \\
\text { Disagree }\end{array}$ \\
\hline Rating: & $\square$ & $\square$ & $\square$ & $\square$ & $\square$ \\
\hline
\end{tabular}

I found this commonality (or lack thereof) presentation method (the way the screen was mirrored on the 'other' screen) visually appealing.

\begin{tabular}{|c|c|c|c|c|c|}
\hline & $\begin{array}{c}\text { Strongly } \\
\text { Agree }\end{array}$ & Agree & Neutral & Disagree & $\begin{array}{l}\text { Strongly } \\
\text { Disagree }\end{array}$ \\
\hline Rating: & $\square$ & $\square$ & $\square$ & $\square$ & $\square$ \\
\hline
\end{tabular}

I found the commonality method (or lack thereof) used got in the way of my content viewing on the tablet.

\begin{tabular}{|c|c|c|c|c|c|}
\hline & $\begin{array}{l}\text { Strongly } \\
\text { Agree }\end{array}$ & Agree & Neutral & Disagree & $\begin{array}{l}\text { Strongly } \\
\text { Disagree }\end{array}$ \\
\hline Rating: & $\square$ & $\square$ & $\square$ & $\square$ & $\square$ \\
\hline
\end{tabular}

I found that the commonality method (or lack thereof) got in the way of viewing the TV material.

\begin{tabular}{|c|c|c|c|c|c|}
\hline & $\begin{array}{l}\text { Strongly } \\
\text { Agree }\end{array}$ & Agree & Neutral & Disagree & $\begin{array}{l}\text { Strongly } \\
\text { Disagree }\end{array}$ \\
\hline Rating: & $\square$ & $\square$ & $\square$ & $\square$ & $\square$ \\
\hline
\end{tabular}

I felt like I had good awareness of what was happening on the TV while looking at the tablet.

\begin{tabular}{lccccc} 
& Strongly & Agree & Neutral & Disagree & Strongly \\
& Agree & & & Disagree \\
Rating: & $\square$ & $\square$ & $\square$ & $\square$ & $\square$ \\
\hline
\end{tabular}

I felt like I had good awareness of what was happening on the tablet while looking at the TV.

\begin{tabular}{|c|c|c|c|c|c|}
\hline & $\begin{array}{l}\text { Strongly } \\
\text { Agree }\end{array}$ & Agree & Neutral & Disagree & $\begin{array}{l}\text { Strongly } \\
\text { Disagree }\end{array}$ \\
\hline Rating: & $\square$ & $\square$ & $\square$ & $\square$ & $\square$ \\
\hline
\end{tabular}




\section{Clip 2 - The Australian Open}

I found that I could take in the content over the two screens at the same time effectively.

\begin{tabular}{|c|c|c|c|c|c|}
\hline & $\begin{array}{c}\text { Strongly } \\
\text { Agree }\end{array}$ & Agree & Neutral & Disagree & $\begin{array}{l}\text { Strongly } \\
\text { Disagree }\end{array}$ \\
\hline Rating: & $\square$ & $\square$ & $\square$ & $\square$ & $\square$ \\
\hline
\end{tabular}

I had to shift my viewing between the screens a lot to take in the TV and tablet content.

\begin{tabular}{|c|c|c|c|c|c|}
\hline & $\begin{array}{c}\text { Strongly } \\
\text { Agree }\end{array}$ & Agree & Neutral & Disagree & $\begin{array}{l}\text { Strongly } \\
\text { Disagree }\end{array}$ \\
\hline Rating: & $\square$ & $\square$ & $\square$ & $\square$ & $\square$ \\
\hline
\end{tabular}

I found this commonality (or lack thereof) presentation method (the way the screen was mirrored on the 'other' screen) visually appealing.

\begin{tabular}{|c|c|c|c|c|c|}
\hline & $\begin{array}{l}\text { Strongly } \\
\text { Agree }\end{array}$ & Agree & Neutral & Disagree & $\begin{array}{l}\text { Strongly } \\
\text { Disagree }\end{array}$ \\
\hline Rating: & $\square$ & $\square$ & $\square$ & $\square$ & $\square$ \\
\hline
\end{tabular}

I found the commonality method (or lack thereof) used got in the way of my content viewing on the tablet.

\begin{tabular}{|c|c|c|c|c|c|}
\hline & $\begin{array}{l}\text { Strongly } \\
\text { Agree }\end{array}$ & Agree & Neutral & Disagree & $\begin{array}{l}\text { Strongly } \\
\text { Disagree }\end{array}$ \\
\hline Rating: & $\square$ & $\square$ & $\square$ & $\square$ & $\square$ \\
\hline
\end{tabular}

I found that the commonality method (or lack thereof) got in the way of viewing the TV material.

\begin{tabular}{|c|c|c|c|c|c|}
\hline & $\begin{array}{c}\text { Strongly } \\
\text { Agree }\end{array}$ & Agree & Neutral & Disagree & $\begin{array}{l}\text { Strongly } \\
\text { Disagree }\end{array}$ \\
\hline Rating: & $\square$ & $\square$ & $\square$ & $\square$ & $\square$ \\
\hline
\end{tabular}

I felt like I had good awareness of what was happening on the TV while looking at the tablet.

\begin{tabular}{|c|c|c|c|c|c|}
\hline & $\begin{array}{c}\text { Strongly } \\
\text { Agree }\end{array}$ & Agree & Neutral & Disagree & $\begin{array}{l}\text { Strongly } \\
\text { Disagree }\end{array}$ \\
\hline Rating: & $\square$ & $\square$ & $\square$ & $\square$ & $\square$ \\
\hline
\end{tabular}

I felt like I had good awareness of what was happening on the tablet while looking at the TV.

\begin{tabular}{lccccc} 
& Strongly & Agree & Neutral & Disagree & Strongly \\
Rating: & Agree & & & Disagree \\
& $\square$ & $\square$ & $\square$ & $\square$ & $\square$ \\
\hline
\end{tabular}




\section{Clip 3 - The VW Scandal}

I found that I could take in the content over the two screens at the same time effectively.

\begin{tabular}{|c|c|c|c|c|c|}
\hline & $\begin{array}{c}\text { Strongly } \\
\text { Agree }\end{array}$ & Agree & Neutral & Disagree & $\begin{array}{l}\text { Strongly } \\
\text { Disagree }\end{array}$ \\
\hline Rating: & $\square$ & $\square$ & $\square$ & $\square$ & $\square$ \\
\hline
\end{tabular}

I had to shift my viewing between the screens a lot to take in the TV and tablet content.

\begin{tabular}{|c|c|c|c|c|c|}
\hline & $\begin{array}{c}\text { Strongly } \\
\text { Agree }\end{array}$ & Agree & Neutral & Disagree & $\begin{array}{l}\text { Strongly } \\
\text { Disagree }\end{array}$ \\
\hline Rating: & $\square$ & $\square$ & $\square$ & $\square$ & $\square$ \\
\hline
\end{tabular}

I found this commonality (or lack thereof) presentation method (the way the screen was mirrored on the 'other' screen) visually appealing.

\begin{tabular}{|c|c|c|c|c|c|}
\hline & $\begin{array}{l}\text { Strongly } \\
\text { Agree }\end{array}$ & Agree & Neutral & Disagree & $\begin{array}{l}\text { Strongly } \\
\text { Disagree }\end{array}$ \\
\hline Rating: & $\square$ & $\square$ & $\square$ & $\square$ & $\square$ \\
\hline
\end{tabular}

I found the commonality method (or lack thereof) used got in the way of my content viewing on the tablet.

\begin{tabular}{|c|c|c|c|c|c|}
\hline & $\begin{array}{c}\text { Strongly } \\
\text { Agree }\end{array}$ & Agree & Neutral & Disagree & $\begin{array}{l}\text { Strongly } \\
\text { Disagree }\end{array}$ \\
\hline Rating: & $\square$ & $\square$ & $\square$ & $\square$ & $\square$ \\
\hline
\end{tabular}

I found that the commonality method (or lack thereof) got in the way of viewing the TV material.

\begin{tabular}{|c|c|c|c|c|c|}
\hline & $\begin{array}{l}\text { Strongly } \\
\text { Agree }\end{array}$ & Agree & Neutral & Disagree & $\begin{array}{l}\text { Strongly } \\
\text { Disagree }\end{array}$ \\
\hline Rating: & $\square$ & $\square$ & $\square$ & $\square$ & $\square$ \\
\hline
\end{tabular}

I felt like I had good awareness of what was happening on the TV while looking at the tablet.

\begin{tabular}{lccccc} 
& Strongly & Agree & Neutral & Disagree & Strongly \\
& Agree & & & Disagree \\
Rating: & $\square$ & $\square$ & $\square$ & $\square$ & $\square$ \\
\hline
\end{tabular}

I felt like I had good awareness of what was happening on the tablet while looking at the TV.

\begin{tabular}{|c|c|c|c|c|c|}
\hline & $\begin{array}{l}\text { Strongly } \\
\text { Agree }\end{array}$ & Agree & Neutral & Disagree & $\begin{array}{l}\text { Strongly } \\
\text { Disagree }\end{array}$ \\
\hline Rating: & $\square$ & $\square$ & $\square$ & $\square$ & $\square$ \\
\hline
\end{tabular}




\section{Clip 4 - Eggheads}

I found that I could take in the content over the two screens at the same time effectively.

\begin{tabular}{|c|c|c|c|c|c|}
\hline & $\begin{array}{c}\text { Strongly } \\
\text { Agree }\end{array}$ & Agree & Neutral & Disagree & $\begin{array}{l}\text { Strongly } \\
\text { Disagree }\end{array}$ \\
\hline Rating: & $\square$ & $\square$ & $\square$ & $\square$ & $\square$ \\
\hline
\end{tabular}

I had to shift my viewing between the screens a lot to take in the TV and tablet content.

\begin{tabular}{lccccc} 
& Strongly & Agree & Neutral & Disagree & Strongly \\
Disagree & & \\
Rating: & $\square$ & $\square$ & $\square$ & $\square$ & $\square$ \\
\hline
\end{tabular}

I found this commonality (or lack thereof) presentation method (the way the screen was mirrored on the 'other' screen) visually appealing.

\begin{tabular}{|c|c|c|c|c|c|}
\hline & $\begin{array}{l}\text { Strongly } \\
\text { Agree }\end{array}$ & Agree & Neutral & Disagree & $\begin{array}{l}\text { Strongly } \\
\text { Disagree }\end{array}$ \\
\hline Rating: & $\square$ & $\square$ & $\square$ & $\square$ & $\square$ \\
\hline
\end{tabular}

I found the commonality method (or lack thereof) used got in the way of my content viewing on the tablet.

\begin{tabular}{|c|c|c|c|c|c|}
\hline & $\begin{array}{l}\text { Strongly } \\
\text { Agree }\end{array}$ & Agree & Neutral & Disagree & $\begin{array}{l}\text { Strongly } \\
\text { Disagree }\end{array}$ \\
\hline Rating: & $\square$ & $\square$ & $\square$ & $\square$ & $\square$ \\
\hline
\end{tabular}

I found that the commonality method (or lack thereof) got in the way of viewing the TV material.

\begin{tabular}{|c|c|c|c|c|c|}
\hline & $\begin{array}{l}\text { Strongly } \\
\text { Agree }\end{array}$ & Agree & Neutral & Disagree & $\begin{array}{l}\text { Strongly } \\
\text { Disagree }\end{array}$ \\
\hline Rating: & $\square$ & $\square$ & $\square$ & $\square$ & $\square$ \\
\hline
\end{tabular}

I felt like I had good awareness of what was happening on the TV while looking at the tablet.

\begin{tabular}{lccccc} 
& Strongly & Agree & Neutral & Disagree & Strongly \\
& Agree & & & Disagree \\
Rating: & $\square$ & $\square$ & $\square$ & $\square$ & $\square$ \\
\hline
\end{tabular}

I felt like I had good awareness of what was happening on the tablet while looking at the TV.

\begin{tabular}{|c|c|c|c|c|c|}
\hline & $\begin{array}{l}\text { Strongly } \\
\text { Agree }\end{array}$ & Agree & Neutral & Disagree & $\begin{array}{l}\text { Strongly } \\
\text { Disagree }\end{array}$ \\
\hline Rating: & $\square$ & $\square$ & $\square$ & $\square$ & $\square$ \\
\hline
\end{tabular}




\section{Post Study Questionnaire}

Your answers here apply to the experiment as a whole and refer to all clips and techniques used.

Have you ever watched any of these specific programmes before? If so, which ones?

$\square \quad$ Yes -

$\square \quad$ No

I found that in Clip 1 (Wild China) the user interface required mental effort to view the content on both screens.

\begin{tabular}{|c|c|c|c|c|c|}
\hline & $\begin{array}{c}\text { Strongly } \\
\text { Agree }\end{array}$ & Agree & Neutral & Disagree & $\begin{array}{l}\text { Strongly } \\
\text { Disagree }\end{array}$ \\
\hline Rating: & $\square$ & $\square$ & $\square$ & $\square$ & $\square$ \\
\hline
\end{tabular}

I found that in Clip 2 (The Australian Open) the user interface required mental effort to view the content on both screens.

\begin{tabular}{|c|c|c|c|c|c|}
\hline & $\begin{array}{c}\text { Strongly } \\
\text { Agree }\end{array}$ & Agree & Neutral & Disagree & $\begin{array}{l}\text { Strongly } \\
\text { Disagree }\end{array}$ \\
\hline Rating: & $\square$ & $\square$ & $\square$ & $\square$ & $\square$ \\
\hline
\end{tabular}

I found that in Clip 3 (The VW Scandal) the user interface required mental effort to view the content on both screens.

$\begin{array}{cccccc} & \text { Strongly } & \text { Agree } & \text { Neutral } & \text { Disagree } & \text { Strongly } \\ \text { Rating: } & \text { Agree } & \square & & & \text { Disagree } \\ \text { R } & \square & \square & \square & \square & \square\end{array}$

I found that in Clip 4 (Eggheads) the user interface required mental effort to view the content on both screens.

$\begin{array}{lccccc} & \text { Strongly } & \text { Agree } & \text { Neutral } & \text { Disagree } & \text { Strongly } \\ \text { Rating: } & \text { Agree } & \square \text { Disagree }\end{array}$

I liked Clip 1 (Wild China) show

\begin{tabular}{|c|c|c|c|c|c|}
\hline & $\begin{array}{c}\text { Strongly } \\
\text { Agree }\end{array}$ & Agree & Neutral & Disagree & $\begin{array}{l}\text { Strongly } \\
\text { Disagree }\end{array}$ \\
\hline Rating: & $\square$ & $\square$ & $\square$ & $\square$ & $\square$ \\
\hline
\end{tabular}


I liked Clip 2 (The Australian Open) show

\begin{tabular}{|c|c|c|c|c|c|}
\hline & $\begin{array}{c}\text { Strongly } \\
\text { Agree }\end{array}$ & Agree & Neutral & Disagree & $\begin{array}{l}\text { Strongly } \\
\text { Disagree }\end{array}$ \\
\hline Rating: & $\square$ & $\square$ & $\square$ & $\square$ & $\square$ \\
\hline
\end{tabular}

I liked Clip 3 (The VW Scandal) show

$\begin{array}{lccccc} & \text { Strongly } & \text { Agree } & \text { Neutral } & \text { Disagree } & \text { Strongly } \\ \text { Agree } & & & & \text { Disagree } \\ \text { Rating: } & \square & \square & \square & \square & \square\end{array}$

I liked Clip 4 (Eggheads) show

\begin{tabular}{lccccc} 
& Strongly & Agree & Neutral & Disagree & Strongly \\
Rating: & Agree & & & Disagree \\
& $\square$ & $\square$ & $\square$ & $\square$ & $\square$ \\
\hline
\end{tabular}

END OF QUESTIONNAIRE - WE WILL NOW CONDUCT A SHORT INTERVIEW. 

Appendix F

\section{Research Consent Forms}




\section{Swansea University - Computer Science Department}

\section{Research Consent Form}

This consent form, a copy of which has been given to you, is only part of the process of informed consent. It should give you the basic idea of what the research is about and what your participation will involve. If you would like more detail about something mentioned here, or information not included here, please ask. Please take the time to read this form carefully and to understand any accompanying information.

\section{Research Project Title}

Dual-screen Usage: Online Survey

\section{Researcher}

Mr Timothy Neate

\section{Experiment Purpose}

The purpose of this online survey is to probe current usage of dual-screen scenarios in terms of attention. We aim to uncover information about how much focus is required for certain second screen interactions, in relation to primary screen content.

\section{Participant Recruitment and Selection}

You were recruited via an online mailing list or similar. You were selected because you had some experience using a second screen while watching TV. To incentivise the experiment, three participants will be awarded vouchers at random - one $£ 50$, and two $£ 25$.

\section{Procedure}

For the survey participants will be asked to first fill out demographics forms. Then, we will ask the experimental questions, which will focus on dual-screen interactions. You will be asked to fill in information about your viewing habits in relation to second screen.

\section{Your Data Collection}

Your demographics data will be stored and kept anonymous throughout the process - for example, participants will not provide their names and only be referred to with numbers.

\section{Data Archiving/Destruction}

All data personal will be made anonymous on collection, and kept private while being analysed. When reported the data will be totally anonymous.

\section{Confidentiality}

All information gathered will be confidential, and participant anonymity will be strictly maintained. Participant numbers will be used instead of names and nothing that may identify the participants 
will be reported in the results, except for anonymous contextually relevant information such as their role.

\section{Likelihood of Discomfort}

There is no likelihood of discomfort or risk associated with participation.

\section{Researcher}

Mr. Timothy Neate is working on his $\mathrm{PhD}$ in the Computer Science Department at the Swansea University. His research considers the design of dual-screen digital content for the television experience. His work is supervised by Professor Matt Jones (Swansea University), and Dr. Michael Evans (BBC R\&D).

The researcher can be contacted in Room 503 (FIT Lab) Faraday Tower, Swansea University, Singleton Park, Swansea, UK. His email address is tdjneate@gmail.com, and his academic website is cs.swan.ac.uk/ cstneate/.

\section{Finding out about Results}

The Participants can find out the results of the study by contacting the researcher after March 2017, or by asking to be updated on the progress of the research by email.

\section{Agreement}

Your continuation with the experiment indicates that you have understood to your satisfaction the information regarding participation in the research project and agree to take part as a participant. In no way does this waive you legal rights nor release the investigators, sponsors, or involved institutions from their legal and professional responsibilities. You are free to not answer specific items or questions in interviews or on questionnaires. You are free to withdraw from the study at any time without penalty. Your continued participation should be as informed as your initial consent, so you should feel free to ask for clarification or new information throughout your participation. If you have further questions concerning matters related to this research, please contact the researcher.

A copy of this consent form has been given to you to keep for your records and reference. 


\section{Swansea University - Computer Science Department}

\section{Research Consent Form}

This consent form, a copy of which has been given to you, is only part of the process of informed consent. It should give you the basic idea of what the research is about and what your participation will involve. If you would like more detail about something mentioned here, or information not included here, please ask. Please take the time to read this form carefully and to understand any accompanying information.

\section{Research Project Title}

Investigating the Impact of Visual Complexity in Companion Content

\section{Researcher}

Mr Timothy Neate

\section{Experiment Purpose}

The purpose of this experiment is to investigate the objective and subjective impact of visual complexity on a second screen when a user is watching television.

\section{Participant Recruitment and Selection}

You are one of 20 participants recruited from Salford University and the BBC after being contacted via a mailing list. Each participant in this study is a willing adult and was chosen on a first come first served basis.

\section{Procedure}

The experiment should take around 40 minutes. First you will be asked to fill out a demographics form, then you will be asked to watch four clips from a variety of television shows, each around 6/7 minutes. The clips will be accompanied by a 'second screen experience' on a tablet computer. This will involve (non-interactive) textual and graphical information. After each clip you will be expected to fill out a short questionnaire to reflect on your experience. Then, upon watching all clips, there will be a final questionnaire to gather your overall experience, as well as a short interview.

\section{You Data Collection}

Some data will be collected during the experiment. In the pre-experiment questionnaire you will be asked some demographics information such as age and gender. Also, during and after the experiment some data will be gathered so that we may best evaluate your experience. You will be filmed during the experiment so that we may infer further conclusions.

\section{Data Archiving/Destruction}

All data personal will be anonymized on collection, kept private while being analysed, and will be destroyed once the results have been reported. With regards to video data, this will be kept securely, and privately throughout the analysis process and will be deleted once inspected. 


\section{Confidentiality}

All information gathered will be confidential, and participant anonymity will be strictly maintained. Participant numbers will be used instead of names and nothing that may identify the participants will be reported in the results.

\section{Likelihood of Discomfort}

There is no likelihood of discomfort or risk associated with participation.

\section{Researcher}

Mr. Timothy Neate is working on his $\mathrm{PhD}$ in the Computer Science Department at the Swansea University. His research considers the design of second screen digital content for the television experience. His work is supervised by Professor Matt Jones (Swansea University), and Dr Michael Evans (BBC R\&D).

The researcher can be contacted in Room 500 Faraday Tower, Swansea University, Singleton Park, Swansea, UK. His email address is tdjneate@gmail.com.

\section{Finding out about Results}

The Participants can find out the results of the study by contacting the researcher after March 2016.

\section{Agreement}

Your signature on this form indicates that you have understood to your satisfaction the information regarding participation in the research project and agree to take part as a participant. In no way does this waive you legal rights nor release the investigators, sponsors, or involved institutions from their legal and professional responsibilities. You are free to not answer specific items or questions in interviews or on questionnaires. You are free to withdraw from the study at any time without penalty. Your continued participation should be as informed as your initial consent, so you should feel free to ask for clarification or new information throughout your participation. If you have further questions concerning matters related to this research, please contact the researcher.

Participant

Investigator/Witness
Date

Date

A copy of this consent form has been given to you to keep for your records and reference. 


\section{Swansea University - Computer Science Department}

\section{Research Consent Form}

This consent form, a copy of which has been given to you, is only part of the process of informed consent. It should give you the basic idea of what the research is about and what your participation will involve. If you would like more detail about something mentioned here, or information not included here, please ask. Please take the time to read this form carefully and to understand any accompanying information.

\section{Research Project Title}

Methods for Second Screen Complexity Control

\section{Researcher \\ Mr Timothy Neate}

\section{Experiment Purpose}

The purpose of this experiment is to investigate the objective and subjective impact of different methods of complexity control on the second screen.

\section{Participant Recruitment and Selection}

You are a participant recruited from Salford University or the BBC after being contacted via a mailing list. Each participant in this study is a willing adult and was chosen on a first come first served basis.

\section{Procedure}

The experiment should take around 40 minutes. First you will be asked to fill out a demographics form, then you will be asked to watch three clips from a variety of television shows, each around 7 minutes in length. The clips will be accompanied by a 'second screen experience' on a tablet computer. This will involve graphical and textual information, and short quizzes related to the show. After each clip you will be asked to fill out a short questionnaire to reflect on your experience. Then, upon watching all three clips, there is a final questionnaire to gather your overall experience. This is all concluded with a short interview and debrief.

\section{Your Data Collection}

Some data will be collected during the experiment. In the pre-experiment questionnaire you will be asked some demographics information such as age and gender. Also, during and after the experiment some data will be gathered so that we may best evaluate your experience. You will be filmed during the experiment so that we may infer further conclusions. In addition, we log data such as gyroscopes and touch data from the device.

\section{Data Archiving/Destruction}


All data personal will be made anonymous on collection, and kept private while being analysed. With regards to video data, this will be kept securely, and privately throughout the analysis process and deleted once fully reported upon.

\section{Confidentiality}

All information gathered will be confidential, and participant anonymity will be strictly maintained. Participant numbers will be used instead of names and nothing that may identify the participants will be reported in the results.

\section{Likelihood of Discomfort}

There is no likelihood of discomfort or risk associated with participation.

\section{Researcher}

Mr. Timothy Neate is working on his PhD in the Computer Science Department at the Swansea University. His research considers the design of second screen digital content for the television experience. His work is supervised by Professor Matt Jones (Swansea University), and Doctor Michael Evans (BBC R\&D).

The researcher can be contacted in Room 503 (FIT Lab) Faraday Tower, Swansea University, Singleton Park, Swansea, UK. His email address is tdjneate@gmail.com, and personal website is cs.swan.ac.uk/ cstneate/.

\section{Finding out about Results}

The Participants can find out the results of the study by contacting the researcher after March 2017, or by asking to be updated on the progress of the research.

\section{Agreement}

Your signature on this form indicates that you have understood to your satisfaction the information regarding participation in the research project and agree to take part as a participant. In no way does this waive you legal rights nor release the investigators, sponsors, or involved institutions from their legal and professional responsibilities. You are free to not answer specific items or questions in interviews or on questionnaires. You are free to withdraw from the study at any time without penalty. Your continued participation should be as informed as your initial consent, so you should feel free to ask for clarification or new information throughout your participation. If you have further questions concerning matters related to this research, please contact the researcher.

Participant

Investigator/Witness

\section{Date}

Date

A copy of this consent form has been given to you to keep for your records and reference. 


\section{Swansea University - Computer Science Department}

\section{Research Consent Form}

This consent form, a copy of which has been given to you, is only part of the process of informed consent. It should give you the basic idea of what the research is about and what your participation will involve. If you would like more detail about something mentioned here, or information not included here, please ask. Please take the time to read this form carefully and to understand any accompanying information.

\section{Research Project Title}

Display Commonalities Focus Group

\section{Researcher \\ Mr Timothy Neate}

\section{Experiment Purpose}

The purpose of this experiment is to discuss our current design space for display commonalities to interested stakeholders at the BBC. We want to understand, of our proposed methods, which are the most feasible from a broadcaster's perspective, with an aim to whittle the options down to 2 or 3 such that we may conduct a systematic user study.

\section{Participant Recruitment and Selection}

You have been specially selected as you are BBC staff engaged in the creation of second-screen content, or are somehow related to the production of related content.

\section{Procedure}

In this focus group the facilitator (Tim) will first be finding out about you by asking each participant to briefly explain his or her role in the creation of content. Then we will progress to give an overview of this focus group, discussing display commonalities. Upon confirming that everyone is up to speed, we will then exhibit our prototypes and await your feedback and get your perspectives. We will then draw out the positives and negatives of each prototype and wrap up with a brief conclusion.

\section{Your Data Collection}

For this focus group we will take some photos for evidence, but mostly we will be using a dictaphone to record the conversations. These will be transcribed for analysis.

\section{Data Archiving/Destruction}

All data personal will be made anonymous on collection, and kept private while being analysed. With regards to video data, this will be kept securely, and privately throughout the analysis process and deleted once fully reported upon. 


\section{Confidentiality}

All information gathered will be confidential, and participant anonymity will be strictly maintained. Participant numbers will be used instead of names and nothing that may identify the participants will be reported in the results, except for anonymous contextually relevant information such as their role.

\section{Likelihood of Discomfort}

There is no likelihood of discomfort or risk associated with participation.

\section{Researcher}

Mr. Timothy Neate is working on his $\mathrm{PhD}$ in the Computer Science Department at the Swansea University. His research considers the design of dual-screen digital content for the television experience. His work is supervised by Professor Matt Jones (Swansea University), and Dr. Michael Evans (BBC R\&D).

The researcher can be contacted in Room 503 (FIT Lab) Faraday Tower, Swansea University, Singleton Park, Swansea, UK. His email address is tdjneate@gmail.com, and his academic website is cs.swan.ac.uk/ cstneate/.

\section{Finding out about Results}

The Participants can find out the results of the study by contacting the researcher after March 2017, or by asking to be updated on the progress of the research by email.

\section{Agreement}

Your signature on this form indicates that you have understood to your satisfaction the information regarding participation in the research project and agree to take part as a participant. In no way does this waive you legal rights nor release the investigators, sponsors, or involved institutions from their legal and professional responsibilities. You are free to not answer specific items or questions in interviews or on questionnaires. You are free to withdraw from the study at any time without penalty. Your continued participation should be as informed as your initial consent, so you should feel free to ask for clarification or new information throughout your participation. If you have further questions concerning matters related to this research, please contact the researcher.

Participant

Investigator/Witness

\section{Date}

\section{Date}

A copy of this consent form has been given to you to keep for your records and reference. 


\section{Swansea University - Computer Science Department}

\section{Research Consent Form}

This consent form, a copy of which has been given to you, is only part of the process of informed consent. It should give you the basic idea of what the research is about and what your participation will involve. If you would like more detail about something mentioned here, or information not included here, please ask. Please take the time to read this form carefully and to understand any accompanying information.

\section{Research Project Title}

Display Commonalities Usability Study

Researcher

Mr Timothy Neate

\section{Experiment Purpose}

The purpose of this experiment is to evaluate the design space for using commonalties in dualscreen TV. By commonalities, we mean duplicating certain elements of one screen on the other. For example, by mirroring the video content of the television on the tablet. During this we encourage you engage with the browser on the second screen, as well as the television.

\section{Participant Recruitment and Selection}

You have been selected from as a person interested in being involved in this experiment.

\section{Procedure}

For this study we will first ask some information about you - we will collect demographics and other experiment related information to enable more in-depth analysis of the data. Then, we will begin the experiment. For this we will play you four short clips while browsing information related to the show. We will use this to test the different commonality methods, compared to a baseline.

\section{Your Data Collection}

For this experiment we will be recording you with a camera. Moreover, we will conduct a short interview. Further, we will collect demographic information about you.

\section{Data Archiving/Destruction}

All data personal will be made anonymous on collection, and kept private while being analyzed. With regards to video data, this will be kept securely, and privately throughout the analysis process and deleted once fully reported upon.

\section{Confidentiality}

All information gathered will be confidential, and participant anonymity will be strictly maintained. Participant numbers will be used instead of names and nothing that may identify the participants will be reported in the results, except for anonymous contextually relevant information such as their role.

\section{Likelihood of Discomfort}


There is no likelihood of discomfort or risk associated with participation.

\section{Researcher}

Mr. Timothy Neate is working on his PhD in the Computer Science Department at the Swansea University. His research considers the design of dual-screen digital content for the television experience. His work is supervised by Professor Matt Jones (Swansea University), and Dr. Michael Evans (BBC R\&D). The researcher can be contacted in Room 503 (FIT Lab) Faraday Tower, Swansea University, Singleton Park, Swansea, UK. His email address is HYPERLINK "mailto:tdjneate@gmail.com" tdjneate@gmail.com, and his academic website is cs.swan.ac.uk/ cstneate/.

\section{Finding out about Results}

The Participants can find out the results of the study by contacting the researcher after March 2017, or by asking to be updated on the progress of the research by email.

\section{Agreement}

Your signature on this form indicates that you have understood to your satisfaction the information regarding participation in the research project and agree to take part as a participant. In no way does this waive you legal rights nor release the investigators, sponsors, or involved institutions from their legal and professional responsibilities. You are free to not answer specific items or questions in interviews or on questionnaires. You are free to withdraw from the study at any time without penalty. Your continued participation should be as informed as your initial consent, so you should feel free to ask for clarification or new information throughout your participation. If you have further questions concerning matters related to this research, please contact the researcher.

Participant

Investigator/Witness

\section{Date}

Date

A copy of this consent form has been given to you to keep for your records and reference. 


\section{Swansea University - Computer Science Department \\ Research Participant's Bill of Rights}

The following is a list of your rights if you participate in a research project organised within the Department of Computer Science at Swansea University.

As a research participant, you have the right:

- To be treated with respect and dignity in every phase of the research.

- To be fully and clearly informed of all aspects of the research prior to becoming involved in it.

- To enter into clear, informed, and written agreement with the researcher prior to becoming involved in the activity. You should sense NO pressure, explicit or otherwise, to sign this contract.

- To choose explicitly whether or not you will become involved in the research under the clearly stated provision that refusal to participate or the choice to withdraw during the activity can be made at any time without penalty to you.

- To be treated with honesty, integrity, openness, and straightforwardness in all phases of the research, including a guarantee that you will not unknowingly be deceived during the course of the research.

- To receive something in return for your time and energy.

- To demand proof that an independent and competent ethical review of human rights and protections associated with the research has been successfully completed.

- To demand complete personal confidentiality and privacy in any reports of the research unless you have explicitly negotiated otherwise.

- To expect that your personal welfare is protected and promoted in all phases of the research, including knowing that no harm will come to you.

- To be informed of the results of the research study in a language you understand.

- To be offered a range of research studies or experiences from which to select, if the research is part of fulfilling your educational or employment goals.

The contents of this bill were prepared by the University of Calgary who examined all of the relevant Ethical Standards from the Canadian Psychological Association's Code of Ethics for Psychologists, 1991 and rewrote these to be of relevance to research participants.

Descriptions of the CPA Ethical Code and the CPA Ethical Standards relevant to each of these rights are available at http://www.cpa.ca/ethics2000.html and http://www.psych.ucalgary.ca/Research/ethics/bill/billcode.html if you would like to examine them.

The complete CPA Ethical Code can be found in Canadian Psychological Association "Companion manual for the Canadian Code of Ethics for Psychologists" (1992). 


\section{Bibliography}

[1] Channel 4. Million Pound Drop App. Online Article: https : / / goo.g1/ xysc66 - Accessed 28/07/16. 2016.

[2] Amazon. Amazon Xray. Online Article : https ://goo.gl/kYUIEe - Accessed: 15/01/17. 2017.

[3] Mary Crawford Annie Beth Fox Jonathan Rosen. 'Distractions, Distractions: Does Instant Messaging Affect College Students' Performance on a Concurrent Reading Comprehension Task?' In: CyberPsychology and Behavior 12.1 (2009). ISSN: 1559-1131.

[4] Edward Anstead, Steve Benford and Robert J. Houghton. 'Many-screen Viewing: Evaluating an Olympics Companion Application'. In: Proceedings of the 2014 ACM International Conference on Interactive Experiences for TV and Online Video. TVX '14. Newcastle Upon Tyne, United Kingdom: ACM, 2014, pp. 103-110. ISBN: 978-1-4503-2838-8. URL: http: //doi.acm.org/ $10.1145 / 2602299.2602304$.

[5] Apprentice: About The Predictor. http://www.bbc.co.uk/apprentice/ series5/about/predictor. shtm1, note $=$ Accessed: 28/07/16.

[6] Mike Armstrong, Matthew Brooks, Anthony Churnside, Michael Evans, Frank Melchior and Matthew Shotton. 'Object-Based Broadcasting: Curation, Responsiveness and User Experience'. In: Proceedings of International Broadcasting Convention. 2014.

[7] Barry Arons. 'A Review of The Cocktail Party Effect'. In: Journal of the American Voice I/O Society 12 (1992), pp. 35-50. 
[8] BBC Blog:The Autumnwatch TV Companion experiment. http://www. bbc . co.uk/blogs/researchanddevelopment/2010/11/the-autumnwatchtv-companion-e.shtml", note $=$ Accessed: 28/07/16.

[9] J. Barrett, M. Hammond and S. Jolly. The Universal Control API Version o.6.o. White Paper 193. BBC, 2011.

[10] Santosh Basapur, Gunnar Harboe, Hiren Mandalia, Ashley Novak, Van Vuong and Crysta Metcalf. 'Field Trial of a Dual Device User Experience for iTV'. In: Proceedings of the gth International Interactive Conference on Interactive Television. EuroITV '11. Lisbon, Portugal: ACM, 2011, pp. 127-136. ISBN: 978-1-4503-0602-7. URL: http : / / doi . acm . org / 10 . 1145 / 2000119. 2000145.

[11] Santosh Basapur, Hiren Mandalia, Shirley Chaysinh, Young Lee, Narayanan Venkitaraman and Crysta Metcalf. 'FANFEEDS: Evaluation of Socially Generated Information Feed on Second Screen As a TV Show Companion'. In: Proceedings of the 1oth European Conference on Interactive Tv and Video. EuroiTV '12. Berlin, Germany: ACM, 2012, pp. 87-96. ISBN: 978-14503-1107-6. URL: http: //doi . acm. org/10 . 1145/2325616 . 2325636.

[12] Johannes W. J. Beentjes and Tom H. A. van der Voort. 'Television viewing versus reading: Mental effort, retention, and inferential learning'. In: Communication Education 42.3 (1993), pp. 191-205. eprint: http : / / dx . doi . org / 10 . $1080 / 03634529309378927$. URL: http: // dx . doi .org/ 10 . $1080 / 03634529309378927$.

[13] L Beltracchi. 'An expert display system and nuclear power plant control rooms'. In: IEEE transactions on nuclear science 35.2 (1988), pp. 991-1000. 
[14] Regina Bernhaupt, Marianna Obrist, Astrid Weiss, Elke Beck and Manfred Tscheligi. 'Trends in the Living Room and Beyond: Results from Ethnographic Studies Using Creative and Playful Probing'. In: Computer Entertainment 6.1 (May 2008), 5:1-5:23. ISSN: 1544-3574. URL: http ://doi . acm . org/10.1145/1350843.1350848.

[15] R.B. Bertolasi. Dual-screen data display terminal for data processing units. US Patent 4,112,423. 1978. URL: http : / / www . google.gg/patents / US4112423.

[16] Meera Blattner, Denise Sumikawa and Robert Greenberg. 'Earcons and Icons: Their Structure and Common Design Principals'. In: Human-Computer Interaction 4 (1989), pp. 11-44.

[17] Jan Bobeth, Johann Schrammel, Stephanie Deutsch, Michael Klein, Mario Drobics, Christina Hochleitner and Manfred Tscheligi. 'Tablet, Gestures, Remote Control?: Influence of Age on Performance and User Experience with iTV Applications'. In: Proceedings of the 2014 ACM International Conference on Interactive Experiences for TV and Online Video. TVX '14. Newcastle Upon Tyne, United Kingdom: ACM, 2014, pp. 139-146. ISBN: 978-14503-2838-8. URL: http://doi.acm.org/10 .1145/2602299. 2602315.

[18] Thomas Booth, Srinivas Sridharan, Ann McNamara, Cindy Grimm and Reynold Bailey. 'Guiding Attention in Controlled Real-world Environments'. In: Proceedings of the ACM Symposium on Applied Perception. SAP '13. Dublin, Ireland: ACM, 2013, pp. 75-82. ISBN: 978-1-4503-2262-1. URL: http :// doi.acm.org/10.1145/2492494.2492508.

[19] Jordan Boyd-Graber, Kimberly Glasgow and Jackie Sauter Zajac. 'Spoiler Alert: Machine Learning Approaches to Detect Social Media Posts with 
Revelatory Information'. In: Proceedings of the 76th ASIS\&T Annual Meeting: Beyond the Cloud: Rethinking Information Boundaries. ASIST '13. Montreal, Quebec, Canada: American Society for Information Science, 2013, 45:145:9. ISBN: o-87715-545-3. URL: http : / / dl . acm . org/citation . cfm? $i d=2655780.2655825$.

[20] S. Adam Brasel and James Gips. 'Media Multitasking Behavior: Concurrent Television and Computer Usage'. In: Cyberpsychology, Behaviour, and Social Networking 14.9 (Mar. 2010), 527-534. URL: http : / / doi . acm . org/ 10 . 1089/cyber. 2010.0350 .

[21] Stephen Brewster, Veli-Pekka Raty and Atte Kortekangas. 'Earcons as a method of providing navigational cues in a menu hierarchy'. In: People and Computers XI. Springer, 1996, pp. 169-183.

[22] Andy Brown, Caroline Jay and Simon Harper. Eye-tracking the dual-screen experience. Tech. rep. 1. Manchester University, 2014.

[23] Andy Brown, Michael Evans, Caroline Jay, Maxine Glancy, Rhianne Jones and Simon Harper. 'HCI over Multiple Screens'. In: $\mathrm{CHI}{ }^{1} 14$ Extended Abstracts on Human Factors in Computing Systems. CHI EA '14. Toronto, Ontario, Canada: ACM, 2014, pp. 665-674. ISBN: 978-1-4503-2474-8. URL: http : / / doi.acm.org/10.1145/2559206.2578869.

[24] Duncan P. Brumby, Helena Du Toit, Harry J. Griffin, Ana Tajadura-Jiménez and Anna L. Cox. 'Working with the Television on: An Investigation into Media Multitasking'. In: Proceedings of the Extended Abstracts of the $32 \mathrm{Nd}$ Annual ACM Conference on Human Factors in Computing Systems. CHI EA '14. Toronto, Ontario, Canada: ACM, 2014, pp. 1807-1812. ISBN: 978-1-45032474-8. URL: http://doi.acm.org/10 .1145/2559206.2581210. 
[25] Marcus Carter, Bjorn Nansen and Martin R. Gibbs. 'Screen Ecologies, Multigaming and Designing for Different Registers of Engagement'. In: Proceedings of the First ACM SIGCHI Annual Symposium on Computer-human Interaction in Play. CHI PLAY '14. Toronto, Ontario, Canada: ACM, 2014, pp. 3746. ISBN: 978-1-4503-3014-5. URL: http : / / doi . acm . org / 10 . 1145 / 2658537.2658686.

[26] Daniel Chen and Roel Vertegaal. 'Using Mental Load for Managing Interruptions in Physiologically Attentive User Interfaces'. In: CHI 'o4 Extended Abstracts on Human Factors in Computing Systems. CHI EA 'o4. Vienna, Austria: ACM, 2004, pp. 1513-1516. ISBN: 1-58113-703-6. URL: http://doi . acm.org/10.1145/985921.986103.

[27] Konstantinos Chorianopoulos, Francisco Javier Burón Fernández, Enrique García Salcines and Carlos de Castro Lozano. 'Delegating the Visual Interface Between a Tablet and a TV'. In: Proceedings of the International Conference on Advanced Visual Interfaces. AVI '10. Roma, Italy: ACM, 2010, pp. 418-418. ISBN: 978-1-4503-0076-6. URL: http : / / doi . acm . org/10 . $1145 / 1842993.1843096$.

[28] Companion Principals: Examples. Online: http://companion . prototype0 . net/examples. Accessed: 14/09/15. 2014.

[29] Jerry L. Cook and Randall M. Jones. 'Texting and Accessing the Web While Driving: Traffic Citations and Crashes Among Young Adult Drivers'. In: Traffic Injury Prevention 12.6 (2011). PMID: 22133329, pp. 545-549. URL: http://dx.doi.org/10.1080/15389588.2011.620999.

[30] Channel Four Television Corporation. Foxes Live: Wild in the City. Online Article : https://goo.gl/kYUIEe - Accessed: 15/01/17. 2014. 
[31] Cédric Courtois and Evelien D'heer. 'Second Screen Applications and Tablet Users: Constellation, Awareness, Experience, and Interest'. In: Proceedings of the 1oth European Conference on Interactive Tv and Video. EuroiTV '12. Berlin, Germany: ACM, 2012, pp. 153-156. ISBN: 978-1-4503-1107-6. URL: http://doi.acm.org/10.1145/2325616.2325646.

[32] Paolo Cremonesi, Roberto Pagano, Stefano Pasquali and Roberto Turrin. 'TV Program Detection in Tweets'. In: Proceedings of the 11th European Conference on Interactive TV and Video. EuroITV '13. Como, Italy: ACM, 2013, pp. 45-54. ISBN: 978-1-4503-1951-5. URL: http://doi . acm.org/10 . $1145 / 2465958.2465960$.

[33] Leon Cruickshank, Emmanuel Tsekleves, Roger Whitham, Annette Hill and Kaoruko Kondo. 'Making Interactive TV Easier to Use: Interface Design for a Second Screen Approach'. In: The Design fournal 20 (2007).

[34] R. Dallimonti. Concurrent overview and detail display system having process control capabilities. US Patent 4,001,807. 1977. URL: https : / /wWw google . $\mathrm{com} / \mathrm{patents/US4001807.}$

[35] David Dearman and Jeffery S. Pierce. 'It's on My Other Computer!: Computing with Multiple Devices'. In: Proceedings of the SIGCHI Conference on Human Factors in Computing Systems. CHI '08. Florence, Italy: ACM, 2008, pp. 767-776. ISBN: 978-1-60558-011-1. URL: http : / / doi . acm . org/ 10 . $1145 / 1357054.1357177$.

[36] M. O. van Deventer, H. Stokking, M. Hammond, J. Le Feuvre and P. Cesar. 'Standards for multi-stream and multi-device media synchronization'. In: IEEE Communications Magazine 54.3 (2016), pp. 16-21. ISSN: 0163-6804. 
[37] Evelien D’heer, Cédric Courtois and Steve Paulussen. 'Everyday Life in (Front of) the Screen: The Consumption of Multiple Screen Technologies in the Living Room Context'. In: Proceedings of the 1oth European Conference on Interactive Tv and Video. EuroiTV '12. Berlin, Germany: ACM, 2012, pp. 195-198. ISBN: 978-1-4503-1107-6. URL: http :// doi . acm. org/10 . $1145 / 2325616.2325654$.

[38] John Dowell, Sylvain Malacria, Hana Kim and Edward Anstead. 'Companion apps for information-rich television programmes: representation and interaction'. In: Personal and Ubiquitous Computing (2015), p. 14. URL: https : //hal.inria.fr/hal-01174692.

[39] Nina Dragutinovic and Divera Twisk. 'Use of mobile phones while drivingeffects on road safety'. In: SWOV Institute, Leidschendam (2005).

[40] Katharina Emmerich, Stefan Liszio and Maic Masuch. 'Defining Second Screen Gaming: Exploration of New Design Patterns'. In: Proceedings of the 11th Conference on Advances in Computer Entertainment Technology. ACE '14. Funchal, Portugal: ACM, 2014, 7:1-7:8. ISBN: 978-1-4503-2945-3. URL: http://doi.acm.org/10.1145/2663806.2663855.

[41] Dillon Eversman, Timothy Major, Mithila Tople, Lauren Schaffer and Janet Murray. 'United Universe: A Second Screen Transmedia Experience'. In: Proceedings of the ACM International Conference on Interactive Experiences for TV and Online Video. TVX '15. Brussels, Belgium: ACM, 2015, pp. 173178. ISBN: 978-1-4503-3526-3. URL: http : / / doi . acm . org / 10 . 1145 / 2745197.2755520 . 
[42] Michael Eysenck and Mark Keane. Cognitive Psychology: A Student's Handbook. 5th ed. Vol. 5. Psychology Press, Taylor and Francis Group, 2005. ISBN: $1-84169-359-6$

[43] Manfred Fahle and Christian Wehrhahn. 'Motion perception in the peripheral visual field'. In: Graefe's Archive Ophthalmology 229 (1991), pp. 430436.

[44] Sanaz Fallahkhair, L. Pemberton and J. Masthoff. 'A dual device scenario for informal language learning: interactive television meets the mobile phone'. In: IEEE international conference on advanced learning technologies: proceedings, 30 August - 1 September 2004, foensuu, Finland. IEEE, 2004, pp. 16-20. ISBN: 9780769521817 .

[45] Leah Findlater and Joanna McGrenere. 'A Comparison of Static, Adaptive, and Adaptable Menus'. In: Proceedings of the SIGCHI Conference on Human Factors in Computing Systems. CHI '04. Vienna, Austria: ACM, 2004, pp. 8996. ISBN: 1-58113-702-8. URL: http: // doi .acm.org/10 . 1145/985692 . 985704 .

[46] Leah Findlater and Joanna McGrenere. 'Impact of Screen Size on Performance, Awareness, and User Satisfaction with Adaptive Graphical User Interfaces'. In: Proceedings of the SIGCHI Conference on Human Factors in Computing Systems. CHI 'o8. Florence, Italy: ACM, 2008, pp. 1247-1256. ISBN: 978-1-60558-o11-1. URL: http : / / doi . acm . org/10 . 1145/1357054 . 1357249.

[47] Alexandre Fleury, Jakob Schou Pedersen and Lars Bo Larsen. 'Evaluating user preferences for video transfer methods from a mobile device to a TV screen'. In: Pervasive and Mobile Computing 9.2 (2013), pp. 228-241. 
[48] Ian Forrester. What is Perceptive Media? 2012. URL: http://www. bbc.co . $\mathrm{uk} / \mathrm{rd} / \mathrm{blog} / 2012 / 07 /$ what-is-perceptive-media.

[49] William J. Friedman and Frank Laycock. 'Children's Analog and Digital Clock Knowledge'. English. In: Child Development 60.2 (1989), pp. 357-371. ISSN: 00093920. URL: http: //WWw. jstor.org/stable/1130982.

[50] David Geerts, Pablo Cesar and Dick Bulterman. 'The Implications of Program Genres for the Design of Social Television Systems'. In: Proceedings of the 1st International Conference on Designing Interactive User Experiences for TV and Video. UXTV '08. Silicon Valley, California, USA: ACM, 2008, pp. 71-80. ISBN: 978-1-60558-100-2. URL: http : / / doi . acm . org / 10 . $1145 / 1453805.1453822$.

[51] David Geerts and Dirk De Grooff. 'Supporting the Social Uses of Television: Sociability Heuristics for Social Tv'. In: Proceedings of the SIGCHI Conference on Human Factors in Computing Systems. CHI 'o9. Boston, MA, USA: ACM, 2009, pp. 595-604. ISBN: 978-1-60558-246-7. URL: http : //doi . acm . org/10.1145/1518701.1518793.

[52] David Geerts, Ishan Vaishnavi, Rufael Mekuria, Oskar van Deventer and Pablo Cesar. 'Are We in Sync?: Synchronization Requirements for Watching Online Video Together.' In: Proceedings of the SIGCHI Conference on Human Factors in Computing Systems. CHI '11. Vancouver, BC, Canada: ACM, 2011, pp. 311-314. ISBN: 978-1-4503-0228-9. URL: http : // doi.acm.org/ $10.1145 / 1978942.1978986$.

[53] David Geerts, Rinze Leenheer, Dirk De Grooff, Joost Negenman and Susanne Heijstraten. 'In Front of and Behind the Second Screen: Viewer and Producer Perspectives on a Companion App'. In: Proceedings of the 2014 ACM 
International Conference on Interactive Experiences for TV and Online Video. TVX '14. Newcastle Upon Tyne, United Kingdom: ACM, 2014, pp. 95-102. ISBN: 978-1-4503-2838-8. URL: http : / / doi . acm.org/10 . 1145/2602299. 2602312.

[54] Google. 'The New Multi-screen World:Understanding Cross-platform Consumer Behaviour'. In: Google, 2012. URL: http://goo.g1/xdbOe1.

[55] Leightman Research Group. Actionable Research on the Broadband, Media and Entertainment Industries. Tech. rep. Leightman Research Group, 2015.

[56] NPD Group. Screen Mirroring Awareness Reaches 40 Percent of Smartphone and Tablet Owners. Online Article: http ://goo.gl/2obWuQ - Accessed: 04/01/16. 2013.

[57] Jonathan Grudin. 'Partitioning Digital Worlds: Focal and Peripheral Awareness in Multiple Monitor Use'. In: Proceedings of the SIGCHI Conference on Human Factors in Computing Systems. Vol. 3. CHI 'o1 1. ACM. 2001, pp. 458465 .

[58] Jan Gugenheimer, Frank Honold, Dennis Wolf, Felix Schüssel, Julian Seifert, Michael Weber and Enrico Rukzio. 'How Companion-Technology can Enhance a Multi-Screen Television Experience: A Test Bed for Adaptive Multimodal Interaction in Domestic Environments'. In: KI - Künstliche Intelligenz 30.1 (2015), pp. 37-44. URL: http : / / dx . doi . org/10 . 1007 / s13218-015-0395-7.

[59] Rebecca Hansson, Peter Ljungstrand and Johan Redström. 'Subtle and Public Notification Cues for Mobile Devices'. In: Proceedings of UbiComp 2001. 2001, pp. 240-246. 
[6o] Simon Harper, Eleni Michailidou and Robert Stevens. 'Toward a Definition of Visual Complexity As an Implicit Measure of Cognitive Load'. In: ACM Transactions on Applied Perception 6.2 (Mar. 2009), 10:1-10:18. ISSN: 15443558. URL: http://doi.acm.org/10 .1145/1498700 . 1498704.

[61] Christopher Heaps and Stephen Handel. 'Similarity and features of natural textures'. In: fournal of Experimental Psychology: Human Perception and Performance 25 (1999), pp. 299-320.

[62] Christian Heath, Marina Jirotka, Paul Luff and Jon Hindmarsh. 'Unpacking collaboration: the interactional organisation of trading in a city dealing room'. In: Computer Supported Cooperative Work (CSCW) 3.2 (1994), pp. $147^{-165}$.

[63] Jan Hess, Benedikt Ley, Corinna Ogonowski, Lin Wan and Volker Wulf. 'Jumping Between Devices and Services: Towards an Integrated Concept for Social Tv'. In: Proceddings of the gth International Interactive Conference on Interactive Television. EuroITV '11. Lisbon, Portugal: ACM, 2011, pp. 1120. ISBN: 978-1-4503-0602-7. URL: http : / / doi . acm . org/10 . 1145 / 2000119.2000122 .

[64] Shawndra Hill, Aman Nalavade and Adrian Benton. 'Social TV: Real-time Social Media Response to TV Advertising'. In: Proceedings of the Sixth International Workshop on Data Mining for Online Advertising and Internet Economy. ADKDD '12. Beijing, China: ACM, 2012, 4:1-4:9. ISBN: 978-1-45031545-6. URL: http://doi.acm.org/10 . 1145/2351356. 2351360.

[65] Charlotte Hoare, Rosie Campbell, Richard Felton and Liam Betsworth. 'Hide and Seek: Exploring Interaction With Smart Wallpaper'. In: Proceedings of the 2015 Annual Symposium on Computer-Human Interaction in Play. CHI 
PLAY '15. London, United Kingdom: ACM, 2015, pp. 129-133. ISBN: 978-14503-3466-2. URL: http://doi.acm.org/10 . 1145/2793107.2793143.

[66] Michael E. Holmes, Sheree Josephson and Ryan E. Carney. 'Visual Attention to Television Programs with a Second-screen Application'. In: Proceedings of the Symposium on Eye Tracking Research and Applications. ETRA '12. Santa Barbara, California: ACM, 2012, pp. 397-400. ISBN: 978-1-4503-12219. URL: http://doi.acm.org/10 .1145/2168556. 2168646.

[67] Christian Holz, Frank Bentley, Karen Church and Mitesh Patel. 'I’M Just on My Phone and They'Re Watching TV": Quantifying Mobile Device Use While Watching Television'. In: Proceedings of the ACM International Conference on Interactive Experiences for TV and Online Video. TVX '15. Brussels, Belgium: ACM, 2015, pp. 93-102. ISBN: 978-1-4503-3526-3. URL: http : //doi.acm.org/10.1145/2745197.2745210.

[68] T. Isobe, M. Fujiwara, H. Kaneta, T. Morita and N. Uratani. 'Development of a TV Reception Navigation System Personalized with Viewing Habits'. In: IEEE Trans. on Consum. Electron. 51.2 (May 2005), pp. 665-674. ISSN: 00983063. URL: http://dx.doi.org/10 . 1109/TCE. 2005 . 1468017.

[69] Caroline Jay, Simon Harper, Andy Brown, Maxine Glancy and Mike Armstrong. 'Attention approximation: from the Web to multi-screen television.' In: TVUX-2013 (2013).

[70] S. Jolly and M. Evans. Improving the Experience of Media in the Connected Home with a New Approach to Inter-device Communication. White Paper 242. British Broadcasting Corporation, 2012. 
[71] Brett R. Jones, Hrvoje Benko, Eyal Ofek and Andrew D. Wilson. 'IllumiRoom: Peripheral Projected Illusions for Interactive Experiences'. In: $A C M$ SIGGRAPH 2013 Emerging Technologies. SIGGRAPH '13. Anaheim, California: ACM, 2013, 7:1-7:1. ISBN: 978-1-4503-2340-6. URL: http : / doi . acm . org/10.1145/2503368.2503375.

[72] Theo Jones. Designing for second screens: The Autumnwatch Companion. http://www.bbc.co.uk/blogs/legacy/researchanddevelopment/ 2011/04/the-autumnwatch-companion---de.shtml. 2011.

[73] Y. C. Ju, Shamsi Iqbal and Eric Horvitz. 'Cars, Calls and Cognition: Investigating Driving and Divided Attention'. In: ACM Conference on Human Factors in Computing Systems (CHI). Association for Computing Machinery, Inc., 2010. URL: https : / / WwW . microsoft . com/en-us / research / publication/cars-calls-and-cognition-investigating-drivingand-divided-attention-2/.

[74] Jan Kallenbach, Silja Narhi and Pirkko Oittinen. 'Effects of Extra Information on TV Viewers' Visual Attention, Message Processing Ability, and Cognitive Workload'. In: Comput. Entertain. 5.2 (Apr. 2007). ISSN: 1544-3574. URL: http://doi.acm.org/10.1145/1279540.1279548.

[75] Tomonari Kamba, Shawn A. Elson, Terry Harpold, Tim Stamper and Piyawadee Sukaviriya. 'Using Small Screen Space More Efficiently'. In: Proceedings of the SIGCHI Conference on Human Factors in Computing Systems. CHI '96. Vancouver, British Columbia, Canada: ACM, 1996, pp. 383-39o. ISBN: o-89791-777-4. URL: http : / / doi . acm . org / 10 . 1145/238386 . 238582 . 
[76] Thierry Karsenti and Aurélien Fievez. The iPad in education: uses, benefits, and challenges. Tech. rep. NASA, 2013.

[77] Hyoung-Gook Kim, Jin Young Kim and Jun-Geol Baek. 'An integrated music video browsing system for personalized television'. In: Expert Systems with Applications 38.1 (2011), pp. 776 -784. ISSN: 0957-4174. URL: http: // www.sciencedirect.com/science/article/pii/s0957417410006603.

[78] Minsun Kim, Jiyeun Lee, Hyebeen Lee, Soyeon Kim, Haemi Jung and KwangHee Han. 'The Color and Blink Frequency of LED Notification Lights and Smartphone Users' Urgency Perception'. In: HCI International 2014 - Posters' Extended Abstracts: International Conference, HCI International 2014, Heraklion, Crete, Greece, June 22-27, 2014. Proceedings, Part II. Ed. by Constantine Stephanidis. Cham: Springer International Publishing, 2014, pp. 621-625. ISBN: 978-3-319-07854-o. URL: http : / / dx . doi .org / 10 . 1007 / 978-3319-07854-0_107.

[79] Peter Kincaid, Robert Fishburne, Richard Rogers and Brad Chissom. Derivation of new readability formulas (Automated Readability Index, Fog Count and Flesch Reading Ease Formula) for Navy enlisted personnel. Tech. rep. 875. United States Naval Education and Training Support Command, 1975.

[8o] Jeremy Klein, Jonathan Freeman, David Harding and Ali Teffahi. Assessing the Impact of Second Screen. Tech. rep. MC/168. Technologia, 2014. URL: http://goo.gl/ZEPDbu.

[81] Clemens N. Klokmose, James R. Eagan, Siemen Baader, Wendy Mackay and Michel Beaudouin-Lafon. 'Webstrates: Shareable Dynamic Media'. In: Proceedings of the 28th Annual ACM Symposium on User Interface Software \&\#38; Technology. UIST '15. Daegu, Kyungpook, Republic of Korea: ACM, 
2015, pp. 280-290. ISBN: 978-1-4503-3779-3. URL: http://doi.acm.org/ $10.1145 / 2807442.2807446$.

[82] Hendrik O. Knoche and M Angela Sasse. 'The Sweet Spot: How People Trade off Size and Definition on Mobile Devices'. In: Proceedings of the 16th ACM International Conference on Multimedia. MM 'o8. Vancouver, British Columbia, Canada: ACM, 2008, pp. 21-30. ISBN: 978-1-60558-303-7. URL: http://doi.acm.org/10.1145/1459359.1459363.

[83] Jerry Kramskoy. Orchestrated Media - Beyond second and third screen. 2011. URL: http : / / www . bbc . co . uk/rd/blog/2011-02-orchestratedmedia-beyond-se- 1 .

[84] Jonathan Lazar, Jinjuan Heidi Feng and Harry Hochheiser. Research Methods in Human-Computer Interaction. Wiley Publishing, 2010. ISBN: 0470723378, 9780470723371 .

[85] Julien Leroy, François Rocca, Matei Mancas and Bernard Gosselin. 'Second Screen Interaction: An Approach to Infer Tv Watcher's Interest Using 3D Head Pose Estimation'. In: Proceedings of the 22Nd International Conference on World Wide Web. WWW' ${ }_{13}$ Companion. Rio de Janeiro, Brazil: ACM, 2013, pp. 465-468. ISBN: 978-1-4503-2038-2. URL: http://doi . acm.org/ $10.1145 / 2487788.2487969$.

[86] Lin Lin, Jennifer Lee and Tip Robertson. 'Reading While Watching Video: The Effect of Video Content on Reading Comprehension and Media Multitasking Ability'. In: Journal of Educational Computing Research 45.2 (July 2011), pp. 183-201. 
[87] Mark Lochrie and Paul Coulton. 'Mobile Phones As Second Screen for TV, Enabling Inter-audience Interaction'. In: Proceedings of the 8th International Conference on Advances in Computer Entertainment Technology. ACE '11. Lisbon, Portugal: ACM, 2011, 73:1-73:2. ISBN: 978-1-4503-0827-4. URL: http://doi.acm.org/10.1145/2071423.2071513.

[88] Kiran Lokhande and Hayley J Davison Reynolds. 'Cognitive workload and visual attention analyses of the air traffic control tower flight data manager (TFDM) prototype demonstration'. In: Proceedings of the Human Factors and Ergonomics Society Annual Meeting. Vol. 56. 1. Sage Publications Sage CA: Los Angeles, CA. 2012, pp. 105-109.

[89] Paul P. Maglio and Christopher S. Campbell. 'Tradeoffs in Displaying Peripheral Information'. In: Proceedings of the SIGCHI Conference on Human Factors in Computing Systems. CHI 'oo. The Hague, The Netherlands: ACM, 2000, pp. 241-248. ISBN: 1-58113-216-6. URL: http://doi . acm. org/10 . $1145 / 332040.332438$.

[90] Gavin Mann, Francesco Venturini, Robin Murdoch, Bikash Mishra, Gemma Moorby and Bouchra Carlier. Digital Video and the Connected Consumer. Tech. rep. Accenture, 2015.

[91] Mark Mann, Anthony Churnside, Andrew Bonney and Frank Melchior. Object-Based Audio Applied to Football Broadcasts. White Paper 272. British Broadcasting Corporation, 2013.

[92] Gary Marsden, Andrew Maunder and Munier Parker. 'People are people, but technology is not technology'. In: Philosophical Transactions of the Royal Society of London A: Mathematical, Physical and Engineering Sciences 366.1881 (2008), pp. 3795-3804. ISSN: 1364-503X. 
[93] Misa T. Maruyama, Scott P. Robertson, Sara K. Douglas, Bryan C. Semaan and Heather A. Faucett. 'Hybrid Media Consumption: How Tweeting During a Televised Political Debate Influences the Vote Decision'. In: Proceedings of the 17th ACM Conference on Computer Supported Cooperative Work \&\#38; Social Computing. CSCW '14. Baltimore, Maryland, USA: ACM, 2014, pp. 1422-1432. ISBN: 978-1-4503-2540-o. URL: http : / / doi . acm . org/10 . $1145 / 2531602.2531719$.

[94] Tara Matthews, Anind K. Dey, Jennifer Mankoff, Scott Carter and Tye Rattenbury. 'A Toolkit for Managing User Attention in Peripheral Displays'. In: Proceedings of the 17th Annual ACM Symposium on User Interface Software and Technology. UIST' 04 . Santa Fe, NM, USA: ACM, 2004, pp. 247-256. ISBN: 1-58113-957-8. URL: http : / / doi . acm . org/10 . 1145/1029632 . 1029676.

[95] Mark Maybury, Warren Greiff, Stanley Boykin, Jay Ponte, Chad McHenry and Lisa Ferro. Personalcasting: Tailored Broadcast News. Tech. rep. The MITRE Corporation, 202 Burlington Road, Bedford, MA, USA.: MITRE, 2004.

[96] Mark McGill, John Williamson and Stephen A. Brewster. 'Mirror, Mirror, on the Wall: Collaborative Screen-mirroring for Small Groups'. In: Proceedings of the 2014 ACM International Conference on Interactive Experiences for TV and Online Video. TVX '14. Newcastle Upon Tyne, United Kingdom: ACM, 2014, pp. 87-94. ISBN: 978-1-4503-2838-8. URL: http: //doi . acm.org/10 . $1145 / 2602299.2602319$.

[97] Mark McGill, John Williamson and Stephen A. Brewster. 'It Takes Two (To Co-View): Collaborative Multi-View TV'. In: Proceedings of the ACM International Conference on Interactive Experiences for TV and Online Video. 
TVX '15. Brussels, Belgium: ACM, 2015, pp. 23-32. ISBN: 978-1-4503-3526-3. URL: http://doi.acm.org/10.1145/2745197.2745199.

[98] Alberto Messina, Francisco Morán Burgos, Marius Preda, Skjalg Lepsoy, Miroslaw Bober, Davide Bertola and Stavros Paschalakis. 'Making Second Screen Sustainable in Media Production: The BRIDGET Approach'. In: Proceedings of the ACM International Conference on Interactive Experiences for TV and Online Video. TVX '15. Brussels, Belgium: ACM, 2015, pp. 155160. ISBN: 978-1-4503-3526-3. URL: http : / / doi . acm . org / 10 . 1145 / 2745197.2755517.

[99] Ulla Metzger and Raja Parasuraman. 'The role of the air traffic controller in future air traffic management: An empirical study of active control versus passive monitoring'. In: Human Factors: The fournal of the Human Factors and Ergonomics Society 43.4 (2001), pp. 519-528.

[10o] Eleni Michailidou, Simon Harper and Sean Bechhofer. 'Visual Complexity and Aesthetic Perception of Web Pages'. In: Proceedings of the 26th Annual ACM International Conference on Design of Communication. SIGDOC 'o8. Lisbon, Portugal: ACM, 2008, pp. 215-224. ISBN: 978-1-60558-083-8. URL: http://doi.acm.org/10.1145/1456536.1456581.

[101] Liz Miller. Breaking Bad Story Sync App. Online Article : https : / / goo . g1/B0 jyCI - Accessed: 28/07/16.

[102] Todd Miller and John Stasko. The InfoCanvas: Information Conveyance through Personalized, Expressive Art. 2001.

[103] Gianmarco Gianmarco De Francisci Morales and Ajay Shekhawat. 'The Future of Second Screen Experience'. In: Proc. of TVUX. ACM. 2013. 
[104] More Than Half of All Households in Japan, US and Europe Will Have Smart TVs by 2019. Online: http://news . ihsmarkit.com/press-release/ more-half-all-households-japan-us-and-europe-will-havesmart-tvs-2019. Accessed: 13/12/16. 2016.

[105] Janet Murray, Sergio Goldenberg, Kartik Agarwal, Tarun Chakravorty, Jonathan Cutrell, Abraham Doris-Down and Harish Kothandaraman. 'Story-map: IPad Companion for Long Form TV Narratives'. In: Proceedings of the 1oth European Conference on Interactive Tv and Video. EuroiTV '12. Berlin, Germany: ACM, 2012, pp. 223-226. ISBN: 978-1-4503-1107-6. URL: http://doi.acm.org/ $10.1145 / 2325616.2325659$.

[106] Elizabeth D. Mynatt. 'Designing with Auditory Icons: How Well Do We Identify Auditory Cues?' In: Conference Companion on Human Factors in Computing Systems. CHI '94. Boston, Massachusetts, USA: ACM, 1994, pp. 269270. ISBN: o-89791-651-4. URL: http: / /doi . acm. org/10 . 1145/259963. 260483.

[107] OFCOM. Adults'Media Useand Attitudes Report. Tech. rep. The MITRE Corporation, 202 Burlington Road, Bedford, MA, USA.: OFCOM, 2014.

[108] Jinoh Oh, Youngchul Sung, Jinha Kim, M. Humayoun, Young-Ho Park and Hwanjo Yu. 'Time-Dependent User Profiling for TV Recommendation'. In: Cloud and Green Computing (CGC), 2012 Second International Conference on. 2012, pp. 783-787.

[109] Aude Oliva, Michael L. Mack, Mochan Shrestha and Angela Peeper. 'Identifying the Perceptual Dimensions of Visual Complexity of Scenes'. In: Proceedings of the Annual Meeting of the Cognitive Science Society. 2004. 
264 Bibliography

[110] Michael C. Patterson. 'A Naturalistic Investigation of Media Multitasking While Studying and the Effects on Exam Performance'. In: Teaching of Psychology 44.1 (2017), pp. 51-57. URL: http : / / dx . doi . org/10 . 1177 / 0098628316677913.

[111] Jennifer Pearson, Tom Owen, Harold Thimbleby and George R. Buchanan. 'Co-reading: investigating collaborative group reading'. In: Proceedings of the 12th ACM/IEEE-CS joint conference on Digital Libraries. JCDL '12. Washington, DC, USA: ACM, 2012, pp. 325-334. ISBN: 978-1-4503-1154-o. URL: http://doi.acm.org/10.1145/2232817.2232876.

[112] ITV Plc. Britain's Got Talent App. the App: https : / / goo.g1/XIb5E2 Accessed: 28/07/16.

[113] Henning Pohl and Roderick Murray-Smith. 'Focused and Casual Interactions: Allowing Users to Vary Their Level of Engagement'. In: Proceedings of the SIGCHI Conference on Human Factors in Computing Systems. CHI '13. Paris, France: ACM, 2013, pp. 2223-2232. ISBN: 978-1-4503-1899-0. URL: http://doi.acm.org/10.1145/2470654.2481307.

[114] Umar Rashid, Miguel A. Nacenta and Aaron Quigley. 'The Cost of Display Switching: A Comparison of Mobile, Large Display and Hybrid UI Configurations'. In: Proceedings of the International Working Conference on Advanced Visual Interfaces. AVI '12. Capri Island, Italy: ACM, 2012, pp. 99106. ISBN: 978-1-4503-1287-5. URL: http : / / doi . acm . org / 10 . 1145 / 2254556.2254577 .

[115] Tim Regan and Ian Todd. 'Media Center Buddies: Instant Messaging around a Media Center'. In: Proceedings of NordiCHI. 2004. 
[116] Katharina Reinecke, Tom Yeh, Luke Miratrix, Rahmatri Mardiko, Yuechen Zhao, Jenny Liu and Krzysztof Z. Gajos. 'Predicting Users' First Impressions of Website Aesthetics with a Quantification of Perceived Visual Complexity and Colorfulness'. In: Proceedings of the SIGCHI Conference on $\mathrm{Hu}$ man Factors in Computing Systems. CHI '13. Paris, France: ACM, 2013, pp. 20492058. ISBN: 978-1-4503-1899-o. URL: http : / / doi . acm . org/10 . 1145 / 2470654.2481281.

[117] Jacob M. Rigby, Duncan P. Brumby, Sandy J.J. Gould and Anna L. Cox. 'Media Multitasking at Home: A Video Observation Study of Concurrent TV and Mobile Device Usage'. In: Proceedings of the 2017 ACM International Conference on Interactive Experiences for TV and Online Video. TVX '17. Hilversum, The Netherlands: ACM, 2017, pp. 3-10. ISBN: 978-1-4503-4529-3. URL: http://doi.acm.org/10.1145/3077548.3077560.

[118] Evan F Risko, Dawn Buchanan, Srdan Medimorec and Alan Kingstone. 'Everyday attention: Mind wandering and computer use during lectures'. In: Computers \& Education 68 (2013), pp. 275-283.

[119] Scott Robertson, Cathleen Wharton, Catherine Ashworth and Marita Franzke. 'Dual Device User Interface Design: PDAs and Interactive Television'. In: Proceedings of the SIGCHI Conference on Human Factors in Computing Systems. CHI '96. Vancouver, British Columbia, Canada: ACM, 1996, pp. 7986. ISBN: o-89791-777-4. URL: http : / / doi . acm . org/10 . 1145/238386. 238408.

[120] Simon Robinson, Céline Coutrix, Jennifer Pearson, Juan Rosso, Matheus Fernandes Torquato, Laurence Nigay and Matt Jones. 'Emergeables: Deformable Displays for Continuous Eyes-Free Mobile Interaction'. In: Pro- 
ceedings of the 2016 CHI Conference on Human Factors in Computing Systems. CHI '16. Santa Clara, California, USA: ACM, 2016, pp. 3793-3805. ISBN: 978-1-4503-3362-7. URL: http : / / doi . acm . org / 10 . 1145 / 2858036 . 2858097.

[121] Aitor Rodriguez-Alsina, Guillermo Talavera, Pilar Orero and Jordi Carrabina. 'Subtitle Synchronization across Multiple Screens and Devices'. In: Sensors 12 (2012), pp. 8710-8731. URL: http: // doi:10.3390/s120708710.

[122] John Rooksby, Mattias Rost, Alistair Morrison, Marek Bell, Mathew Chalmers and Timothy Smith. 'Practices of Parallel Media: Using Mobile Devices When Watching Television'. In: CSCW - Designing with Users for Domestic Environments: Methods, Challenges and Lessons Learned. ACM. 2014.

[123] Jacqueline Sachs. 'Memory in reading and listening to discourse'. English. In: Memory and Cognition 2.1 (1974), pp. 95-100. ISSN: 0090-502X. URL: http : //dx.doi.org/10.3758/BF03197498.

[124] Stephanie Santosa and Daniel Wigdor. 'A Field Study of Multi-device Workflows in Distributed Workspaces'. In: Proceedings of the 2013 ACM International foint Conference on Pervasive and Ubiquitous Computing. UbiComp '13. Zurich, Switzerland: ACM, 2013, pp. 63-72. ISBN: 978-1-4503-1770-2. URL: http://doi.acm.org/10.1145/2493432 . 2493476.

[125] Kelly L. Schmitt, Kimberly Duyck Woolf and Daniel R. Anderson. 'Viewing the Viewers: Viewing Behaviors by Children and Adults During Television Programs and Commercials'. In: Journal of Communication 53.2 (2003), pp. 265-281. ISSN: 1460-2466. URL: http : / / dx . doi . org / 10 . $1111 / \mathrm{j}$. $1460-2466.2003 . t b 02590 . x$. 
[126] Jose Shelton and Gideon Praveen Kumar. 'Comparison between Auditory and Visual Simple Reaction Times'. In: Neuroscience and Medicine 1 (2010), pp. $30-32$.

[127] Jennifer G. Sheridan, Jan Borchers, Rafael Ballagas and Michael Rohs. 'The Smart Phone: A Ubiquitous Input Device'. In: IEEE Pervasive Computing 5.undefined (2006), pp. 70-77. ISSN: 1536-1268.

[128] Hyoseop Shin, Minsoo Lee and Eun Yi Kim. 'Personalized digital TV content recommendation with integration of user behavior profiling and multimodal content rating'. In: Consumer Electronics, IEEE Transactions on $55 \cdot 3$ (2009), pp. 1417-1423. ISSN: 0098-3063.

[129] Daniel Simons and Daniel Levin. 'Change Blindness'. In: Trends in Cognitive Sciences 7.1 (1997), pp. 261-267.

[130] Han Sloetjes and Peter Wittenburg. 'Annotation by Category: ELAN and ISO DCR.' In: LREC. 2008.

[131] Barry Smyth and Paul Cotter. 'Personalized electronic program guides for digital TV'. In: Ai Magazine 22.2 (2001), p. 89.

[132] Barbara G Tabachnick, Linda S Fidell and Steven J Osterlind. 'Using multivariate statistics'. In: (2001).

[133] Take this lollipop. http://www . takethislollipop . com/, note = Accessed: $28 / 07 / 16$.

[134] Emmanuel Tsekleves, Roger Whitham, Koko Kondo and Annette Hill. 'Bringing the Television Experience to Other Media in the Home: An Ethnographic Study'. In: Proceedings of the Seventh European Conference on European Interactive Television Conference. EuroITV'o9. Leuven, Belgium: ACM, 2009, 
pp. 201-210. ISBN: 978-1-60558-340-2. URL: http : / / doi . acm . org / 10 . $1145 / 1542084.1542125$.

[135] Melina R. Uncapher, Monica K. Thieu and Anthony D. Wagner. In: ().

[136] Christian Valuch, Ulrich Ansorge, Shelley Buchinger, Aniello Raffaele Patrone and Otmar Scherzer. 'The Effect of Cinematic Cuts on Human Attention'. In: Proceedings of the 2014 ACM International Conference on Interactive Experiences for TV and Online Video. TVX '14. Newcastle Upon Tyne, United Kingdom: ACM, 2014, pp. 119-122. ISBN: 978-1-4503-2838-8. URL: http://doi.acm.org/10.1145/2602299.2602307.

[137] Anna Van Cauwenberge, Gabi Schaap and Rob Van Roy. '"TV No Longer Commands Our Full Attention": Effects of Second-screen Viewing and Task Relevance on Cognitive Load and Learning from News'. In: Computers in Human Behavior 38 (Sept. 2014), pp. 100-109. ISSN: 0747-5632. URL: http : //dx.doi.org/10.1016/j.chb.2014.05.021.

[138] Jeroen Vanattenhoven and David Geerts. 'Second-screen use in the home: an ethnographic study.' In: Proceedings 3 rd international workshop on future television. EuroITV 2012. Berlin: Springer, 2013, pp. 162-173. URL: https : //1irias.kuleuven.be/bitstream/123456789/350723/3/AdjProc_ EuroITV2012.pdf.

[139] Radu-Daniel Vatavu. 'There's a World Outside Your TV: Exploring Interactions Beyond the Physical TV Screen'. In: Proceedings of the 11th European Conference on Interactive TV and Video. EuroITV '13. Como, Italy: ACM, 2013, pp. 143-152. ISBN: 978-1-4503-1951-5. URL: http: //doi.acm.org/ $10.1145 / 2465958.2465972$. 
[140] Radu-Daniel Vatavu and Matei Mancas. 'Visual Attention Measures for Multi-screen TV'. In: Proceedings of the 2014 ACM International Conference on Interactive Experiences for TV and Online Video. TVX '14. Newcastle Upon Tyne, United Kingdom: ACM, 2014, pp. 111-118. ISBN: 978-1-45032838-8. URL: http://doi.acm.org/10.1145/2602299.2602305.

[141] Vinoba Vinayagamoorthy, Rajiv Ramdhany and Matt Hammond. 'Enabling Frame-Accurate Synchronised Companion Screen Experiences'. In: Proceedings of the ACM International Conference on Interactive Experiences for TV and Online Video. TVX '16. Chicago, Illinois, USA: ACM, 2016, pp. 8392. ISBN: 978-1-4503-4067-0. URL: http : / / doi . acm . org / 10 . 1145 / 2932206.2932214.

[142] Claudia Wallis. The impacts of media multitasking on children's learning and development. Tech. rep. Leland Stanford Junior University, 2010.

[143] David Warnock, Marilyn McGee-Lennon and Stephen Brewster. 'The Role of Modality in Notification Performance'. In: Human-Computer Interaction - INTERACT 2011: 13th IFIP TC 13 International Conference, Lisbon, Portugal, September 5-9, 2011, Proceedings, Part II. Ed. by Pedro Campos, Nicholas Graham, Joaquim Jorge, Nuno Nunes, Philippe Palanque and Marco Winckler. Berlin, Heidelberg: Springer Berlin Heidelberg, 2011, pp. 572-588. ISBN: 978-3-642-23771-3. URL: http : / / dx . doi . org/10 . 1007 / 978-3-64223771-3_43.

[144] Mark Weiser. 'The computer for the 21st century'. In: Scientific american 265.3 (1991), pp. 94-104. 
[145] Mark Weiser. 'The Computer for the 21st Century'. In: SIGMOBILE Mob. Comput. Commun. Rev. 3.3 (July 1999), pp. 3-11. ISSN: 1559-1662. URL: http : //doi.acm.org/10.1145/329124.329126.

[146] Tom Williams. New Antiques Roadshow play-along app. http : / /www. bbc . co.uk/blogs/internet/posts/antiques_roadshow_play-along_ a. 2013 .

[147] Robert Andrew Wilson and Frank C Keil. The MIT encyclopedia of the cognitive sciences: Attention. 2001.

[148] Craig Wisneski, Hiroshi Ishii, Andrew Dahley, Matt Gorbet, Scott Brave, Brygg Ullmer and Paul Yarin. 'Ambient Displays: Turning Architectural Space into an Interface between People and Digital Information'. In: Proceedings of the First International Workshop on Cooperative Buildings. Springer, 1998.

[149] Jishuo Yang and Daniel Wigdor. 'Panelrama: Enabling Easy Specification of Cross-device Web Applications'. In: Proceedings of the SIGCHI Conference on Human Factors in Computing Systems. CHI '14. Toronto, Ontario, Canada: ACM, 2014, pp. 2783-2792. ISBN: 978-1-4503-2473-1. URL: http : //doi.acm.org/10.1145/2556288.2557199.

[150] Fang You, Jian-Min Wang, Han Cao, Ting Xie and Ze-Long Tang. 'Social TV EPG Interaction Design for Multi-screen Environment'. In: Green Computing and Communications (GreenCom), 2013 IEEE and Internet of Things (iThings/CPSCom), IEEE International Conference on and IEEE Cyber, Physical and Social Computing. 2013, pp. 758-763. 
[151] Mikel Zorrilla, Njål Borch, François Daoust, Alexander Erk, Julián Flórez and Alberto Lafuente. 'A Web-based distributed architecture for multi-device adaptation in media applications'. In: Personal and Ubiquitous Computing 19.5 (2015), pp. 803-820. URL: http : / dx . doi . org/10 . 1007 / s00779$015-0864-\mathrm{x}$. 